\title{
Traveling Wave Laser Model for Circuit Simulation
}

\author{
by
}

John H. Rasmussen

A thesis submitted to the Faculty of Graduate and Postdoctoral Affairs in partial fulfillment of the requirements for the degree of

\author{
Master of Applied Science
}

in

Electrical and Computer Engineering

\author{
Carleton University \\ Ottawa, Ontario \\ (C) 2016 \\ John H. Rasmussen
}




\section{Abstract}

This thesis examines current modeling of lasers with the aim to justify the Traveling Wave Model (TWM) as the best alternative for an accurate laser model that is not too costly computationally. The goal is not just to have the best model but to have one that can be incorporated into a SPICE-like optical simulator for commercial application. After reviewing other models with specific attention paid to the classic Rate Equation Model (REM) and briefly overviewing the Transmission Line Model (TLM), it presents the TWM details. This model is compared with the REM and we discover that even with enhancements made to the REM once we require a more accurate and detailed model the TWM provides a simpler, more physical model that is computationally comparable and more easily refined. 


\section{Acknowledgements}

This research was supported in part by Natural Sciences and Engineering Research Council of Canada (NSERC), Canadian Institute for Photonic Innovations (CIPI), a Network of Centres of Excellence (NCE) and Optiwave Inc.; their support is gratefully acknowledged.

I would like to thank Tom Smy for his patient guidance through this project. 


\section{Contents}

$\begin{array}{ll}\text { Abstract } & \text { i }\end{array}$

$\begin{array}{ll}\text { Acknowledgements } & \text { ii }\end{array}$

Glossary $\quad$ xiii

1 Introduction $\quad 1$

1.1 Laser Models . . . . . . . . . . . . . . . . . . . . . . . . . . . . . 1

1.2 Thesis Motivation . . . . . . . . . . . . . . . . . . 1

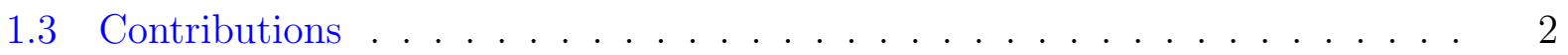

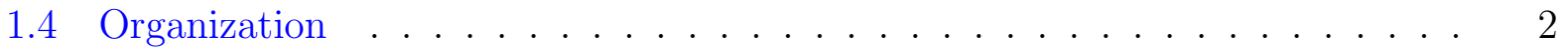

2 Laser Operation $\quad 3$

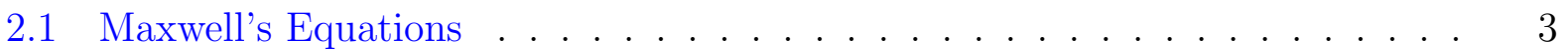

2.1.1 Phasor Form . . . . . . . . . . . . . . . . . . 3

2.1.2 Wave Propagation in Dielectrics . . . . . . . . . . . . . . . . . 4

2.1.3 Waveguide Modes . . . . . . . . . . . . . . . . . . . 5

2.1.4 Ridge Waveguide . . . . . . . . . . . . . . . . . . . . 7

2.1.5 Lossy Waveguide . . . . . . . . . . . . . . . . . . . . 8

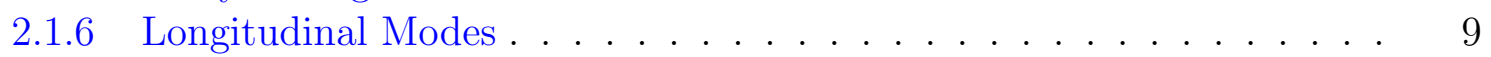

2.2 Elements of a Laser . . . . . . . . . . . . . . . . . . . . . . . . . . 10

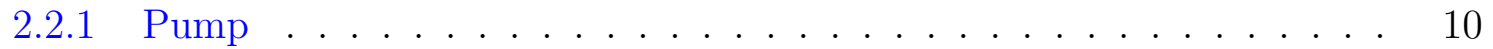

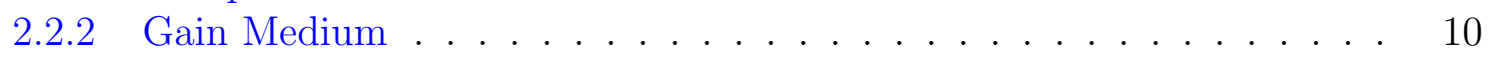

2.2 .3 Optical Feedback . . . . . . . . . . . . . . . . . . . . 11

2.3 Semiconductor Lasers . . . . . . . . . . . . . . . . . . . . . . . . . . . . 12

2.3.1 Physical Structure . . . . . . . . . . . . . . . . . . 12

2.3.2 Band Structure . . . . . . . . . . . . . . . . . . . . . . . . . . . . . . . . . 13

2.3.3 Linewidth . . . . . . . . . . . . . . . . . . . . 14

2.3.4 Waveguide Structure . . . . . . . . . . . . . . . . . 15

2.3.5 Spontaneous Emission . . . . . . . . . . . . . . . . . . . 15

2.3.6 Resonant Cavity . . . . . . . . . . . . . . . . . . . 16

2.3.7 Laser Modes . . . . . . . . . . . . . . . . . . . . . . 17

2.3.8 Electrical Properties . . . . . . . . . . . . . . . 18 
2.4 Carrier Density . . . . . . . . . . . . . . . . . . . . . . . . . . . . 19

2.5 Dispersion . . . . . . . . . . . . . . . . . . . . . . . . . . . . . . 19

2.6 Henry Factor $\ldots \ldots \ldots \ldots$

3 Laser Modeling 23

3.1 Complex Envelope . . . . . . . . . . . . . . . . . . . . . . . . 23

3.1 .1 Timing Reference . . . . . . . . . . . . . . . . . . . . . . 24

3.1 .2 Calculating Envelope Phase . . . . . . . . . . . . . . . . . 25

3.1 .3 Envelope Phase and Time Delay . . . . . . . . . . . . . . . . . 25

3.1 .4 Carriers not in Phase . . . . . . . . . . . . . . . . . 25

3.1.5 Different Carrier Frequencies . . . . . . . . . . . . . . . . . . 26

3.2 Rate Equation Model . . . . . . . . . . . . . . . . . . . . . . . 27

3.3 Rate Equation Model Basic Dynamics . . . . . . . . . . . . . . . . . . . . 29

3.4 Rate Equation Model With Gain Compression . . . . . . . . . . . . . . . . 34

3.5 Rate Equation Model With Phase . . . . . . . . . . . . . . . . . . . 35

3.5.1 Chirp and Henry Factor $\alpha_{H} \ldots \ldots \ldots \ldots \ldots$

3.6 Multi-mode Rate Equation Model . . . . . . . . . . . . . . . . . . . . 36

3.7 Transmission Line Model Fundamentals . . . . . . . . . . . . . . . . . . . . 43

3.8 Traveling Wave Model Fundamentals . . . . . . . . . . . . . . . . . . . . 44

4 Circuit Modeling With Laser Elements 46

4.1 Modified Nodal Analysis Overview . . . . . . . . . . . . . . . . . 46

4.2 Integration with Modified Nodal Analysis Simulation Engine . . . . . . . . 56

5 Traveling Wave Model $\quad \mathbf{5 8}$

5.1 Simplified Traveling Wave Model . . . . . . . . . . . . . . . . . 59

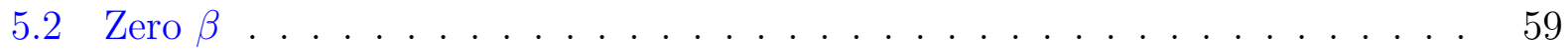

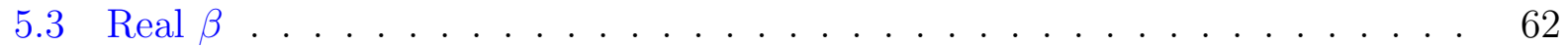

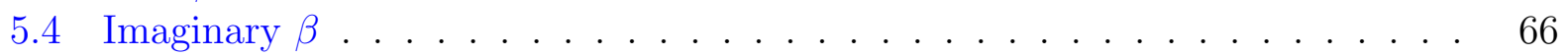

5.5 Finite-Difference Time-Domain Method . . . . . . . . . . . . . . . 69

5.5 .1 Algebraic Difference Equations _... . . . . . . . . . . . . . . . 69

5.5 .2 Solving a Differential Equation . . . . . . . . . . . . . . 71

5.5 .3 Finite-Difference in Time and Space . . . . . . . . . . . . . . . . . 73

5.5.4 Upwind Finite Difference Scheme . . . . . . . . . . . . . . . . 73

5.6 Discretization . . . . . . . . . . . . . . . . . . . . . . 74

5.7 Traveling Wave Model with Dispersion . . . . . . . . . . . . . 76

5.8 Traveling Wave Model with Advanced Terms . . . . . . . . . . . . . . . . 79

5.9 Traveling Wave Model Resonant Cavity and Detuning . . . . . . . . . . . 79

6 Traveling Wave Laser Model $\quad 81$

6.1 Model Slice . . . . . . . . . . . . . . . . . . . . . . . . . . . . . . 82

6.1 .1 Propagation Examples . . . . . . . . . . . . . . . . . . . 83

6.2 Field Distributions . . . . . . . . . . . . . . . . . . . . 85 
7 Comparison of TWM with REM $\quad 88$

7.1 Carrier and Photon Densities _. . . . . . . . . . . . . . . . 88

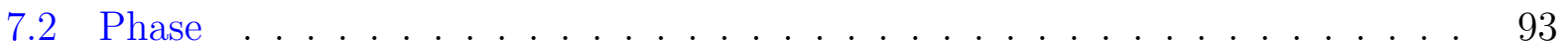

7.3 Henry Factor . . . . . . . . . . . . . . . . . . . . . . . . . . . . 96

7.4 Computational Cost . . . . . . . . . . . . . . . . . . . . 110

7.5 Summary . . . . . . . . . . . . . . . . . . . 113

8 Conclusions $\quad 114$

$\begin{array}{lr}\text { Bibliography } & 116\end{array}$

$\begin{array}{lr}\text { A Interference and Delay through a waveguide } & 120\end{array}$

A.1 Calculating Phase . . . . . . . . . . . . . . . . . . . . 121

A.2 Example: Phase Change Due to Full-wavelength Carrier Delay . . . . . . . . 121

A.3 Example: Phase Change Due to Half-wavelength Carrier Delay . . . . . . . . 122

A.4 Phase change due to arbitrary carrier delay . . . . . . . . . . . . . . . . 124

A.5 Phase change due to different carrier frequencies . . . . . . . . . . . . . . . 125

A.6 Interference and Mode Generation in a Resonant Cavity . . . . . . . . . . . 126

$\begin{array}{lr}\text { B Counter Rotating Waves } & 128\end{array}$

C Multi-mode Rate Equation Model Phase 133

C.1 Multi-mode Phase . . . . . . . . . . . . . . . . . . . . 133

C.1.1 Symmetrical Modes . . . . . . . . . . . . . . . . . 133

C.1.2 Mode Initial Phase . . . . . . . . . . . . . . . . . . . . . 134

C.1.3 Mode Amplitudes . . . . . . . . . . . . . . . . . . 134

D Henry Rate Equations $\quad 141$

D.1 Rate Equations for Photons and Phase . . . . . . . . . . . . . . . . . . 141

E Derivation of Henry Photon and Phase Equations 142

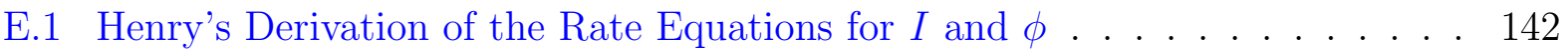

E.1.1 Net Gain . . . . . . . . . . . . . . . . . . . . . . . . . . . . 143

E.2 Derivations . . . . . . . . . . . . . . . . . . . 145

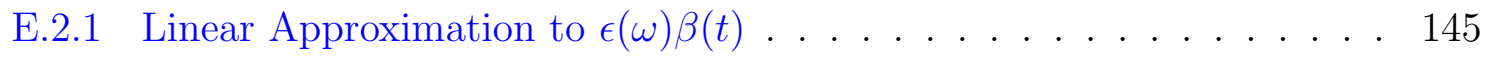

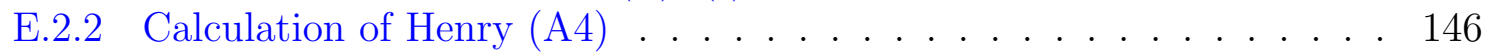

E.2.3 Calculation of Henry (A4) with constant $\varepsilon$. . . . . . . . . . . . . . 149

E.2.4 Calculation of Henry (A7): Relation to $\epsilon$ and $n$. . . . . . . . . . 149

E.2.5 Assume start at threshold . . . . . . . . . . . . . . . . . . . . . 149

E.2.6 Calculation of Henry (A9) . . . . . . . . . . . . . . . . . . . . . 149

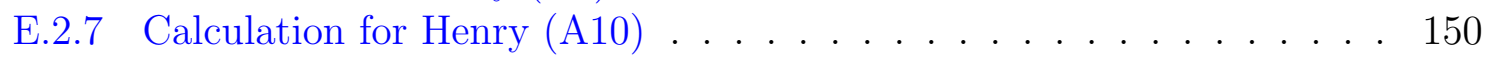

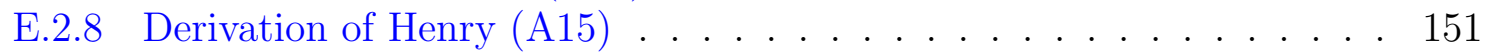

E.2.9 Calculation of Henry (A16) . . . . . . . . . . . . . . . . 152 
E.2.10 Calculation of Henry (A17) . . . . . . . . . . . . . . . . 153

E.2.11 Net Gain or Loss and the Complex Index of Refraction . . . . . . . 153

$\begin{array}{ll}\text { F Henry Factor } & 155\end{array}$

F.1 Derivation of Henry Factor . . . . . . . . . . . . . . . . . . 155

F.2 Henry Factor Example . . . . . . . . . . . . . . . . . . . . 156

F.3 Chirp . . . . . . . . . . . . . . . . . . . . 156

F.4 Change in laser frequency due to change in real index of refraction . . . . . 157

$\begin{array}{lr}\text { G Derivation of TWM from Maxwell's Equations } & 158\end{array}$

G.1 Wave Traveling Right . . . . . . . . . . . . . . . . . . . . . . . . 158

G.2 Wave Traveling Left . . . . . . . . . . . . . . . . . . . . . 159

G.3 Group Velocity . . . . . . . . . . . . . . . . . . . . . . . . . . 160

$\begin{array}{lr}H \text { Derivation of Rate Equations for REM } & 161\end{array}$

H.1 Classic Rate Equations . . . . . . . . . . . . . . . . . . . . . . . . . . . . 161

H.1.1 Optical Power Gain and Loss Parmameters . . . . . . . . . . . . . . 162

$\begin{array}{lr}\text { I Derivation of REM from TWM } & 164\end{array}$

I.1 Carrier equation . . . . . . . . . . . . . . . . . . . . . . . . . . . . . . . . . 164

I.2 Photon equation . . . . . . . . . . . . . . . . . . . . . . . . . 165

I.2.1 Forward Traveling Wave and Material Response . . . . . . . . . . . . 165

I.2.2 Forward Traveling Mode . . . . . . . . . . . . . . . . . . . . . . . . . 165

I.2.3 Forward Traveling Photon Mode Density . . . . . . . . . . . . . 167

I.2.4 S Equation . . . . . . . . . . . . . . . . . . . . . . . . . . . 172

I.3 Phase equation . . . . . . . . . . . . . . . . . . . . . . . . . . . . . . 172

I.4 Issues . . . . . . . . . . . . . . . . . . . . . . . . . . . . . 173

$\begin{array}{lr}\text { J Useful Wave Relations } & 175\end{array}$

J.1 Index of Refraction . . . . . . . . . . . . . . . . . . . . . . . 175

J.1.1 No Damping. . . . . . . . . . . . . . . . . . . . . . . . . 175

J.2 Dispersion . . . . . . . . . . . . . . . . . . . . . . . . . . . . 178

J.2.1 Gas . . . . . . . . . . . . . . . . . . . . . . . . . . . 178

J.2.2 Transparent Substances . . . . . . . . . . . . . . . . . . . . . . . . 178

J.2.3 Opaque Materials . . . . . . . . . . . . . . . . . . . . . . . . . 178

J.2.4 Damping and Multiple Oscillators . . . . . . . . . . . . . 178

$\begin{array}{lr}\text { K Miscellaneous Derivations } & 180\end{array}$

K.1 Classic Photon Lifetime . . . . . . . . . . . . . . . . . . . . . 180 


\section{List of Figures}

2.1 Plot of $E$ field in a lossy medium traveling in $+z$ direction. Shown at two times: $t=0$ and $t=d t$. The field is attenuated as it penetrates the medium.

2.2 Rectangular waveguide filled with a homogeneous isotropic medium. Note that the length is infinite. . . . . . . . . . . . . . . . . . . 5

2.3 Laser diode heterojunction structure. Top and bottom layers are metal contacts connected to a voltage source. With infinite length and width this is a modeled as a slab waveguide but with a finite width becomes a ridge waveguide. 7

2.4 TE modes of symmetrical ridge waveguide. . . . . . . . . . . . . 8

2.5 Cross-section of a rectangular dielectric waveguide. All index regions could be different and we could ignore the metal at the top and bottom for a general case but it will likely be of this form. . . . . . . . . . . . . . .

2.6 Cross-sectional view of transverse field patterns and intensities for rectangular dielectric waveguide. . . . . . . . . . . . . . . . . 9

2.7 Ridge waveguide approximated by a rectangular dielectric waveguide. The dielectric material above the waveguide for the laser active layer has been removed. . . . . . . . . . . . . . . . . . . 9

2.8 Left: Absorption. Middle: Spontaneous emission. Right: Stimulated emission. 11

2.9 Cascade of initially spontaneous and then stimulated emission. There is always a small amount of spontaneous emission, but with a gain medium stimulated emission quickly takes over. Note that although the stimulated emission is shown growing in one direction it will build in both directions. . . . . . . . 11

2.10 Physical structure of basic laser. . . . . . . . . . . . . . . . . . . . 12

2.11 Semiconductor laser Density of States (DOS). The two energy levels of laser are the Conduction Band (CB) and the valence band (VB). The electron drops from $\mathrm{CB}$ to $\mathrm{VB}$ emitting a photon as spontaneous emission. . . . . . . . . 13

2.12 Physical structure of a slab semiconductor laser made on a single IC. . . . . 13

2.13 Semiconductor laser physical diagram. . . . . . . . . . . . . . . . . . 14

2.14 Typical semiconductor laser band structure. Carriers are confined by bandgap so they have lots of time to recombine emitting photons. . . . . . . . . . . . 14

2.15 Semiconductor laser emission intensity modeled as a Lorentzian. . . . . . . . 15 
2.16 Semiconductor laser band diagram under forward bias showing how the layers of material with different indexes of refraction also create a waveguide for photon confinement. . . . . . . . . . . . . . . . . . . . . . . . . . . . .

2.17 Photons are confined to the active layer. . . . . . . . . . . . . . 16

2.18 Semiconductor laser allowed wavelengths. The spacing between these is $2 L$. . 17

2.19 Semiconductor laser relative intensity of the allowed wavelengths. . . . . . . 17

2.20 Semiconductor laser symbol — same as an LED. . . . . . . . . . . . . . . . 18

2.21 Semiconductor laser compact model. . . . . . . . . . . . . . . . . . . . . . 19

2.22 Spread of pulse in time domain due to dispersive medium. Same pulse moving in time. Shortens and widens as it moves. . . . . . . . . . . . . . . 20

2.23 Henry factor $\left(\alpha_{H}\right)$ over frequency for sample index of refraction. . . . . . . . 22

3.1 Active layer showing fields, confinement factor $(\Gamma) . A$ is area of field. $\Gamma A$ is area of field interacting with carriers. . . . . . . . . . . . . 27

3.2 REM current pulse simulation, $N$ starting at $N_{t r} . N$ and $S$ have units $\mathrm{cm}^{-3}$.

3.3 Transient plots of REM DE terms during system startup which then settles at threshold. Carrier and photon densities $N$ and $S$ units are $\mathrm{cm}^{-3}$. . . .

3.4 Plot of REM DE $N$ equation terms during system startup which then settles at threshold. Carrier density $N$ units are $\mathrm{cm}^{-3} \ldots \ldots . . . . . . .$.

3.5 Transient plots of REM DE terms during system startup which then settles at threshold. $S$ terms. Photon density $S$ units are $\mathrm{cm}^{-3} \ldots \ldots \ldots$

3.6 Transient plots of REM DE terms during system startup which then settles at threshold. $N$ equation terms with $I$ and $N$ summed. Carrier density $N$ units are $\mathrm{cm}^{-3}$. . . . . . . . . . . . . . . . . . .

3.7 Transient plots of REM DE terms during system startup which then settles at threshold. $S$ derivative terms. Photon density $S$ units are $\mathrm{cm}^{-3} \ldots . .$.

3.8 Transient plots of REM DE terms during system startup which then settles at threshold. $N$ derivative terms. Carrier density $N$ units are $\mathrm{cm}^{-3}$. . . . .

3.9 REM current pulse simulation, $N$ starting at $N_{t r}, \epsilon=5 \times 10^{-17} . N$ and $S$ have units $\mathrm{cm}^{-3}$ while current is in Amps and phase in radians. . . . . . . .

3.10 REM current pulse simulation, $N$ starting at $N_{t r}$ and showing phase and chirp. $N$ and $S$ have units $\mathrm{cm}^{-3}$ while current is in Amps and phase in radians. . .

3.11 Multi-mode spectrum. Left: spectrum of emission. Middle: Allowed laser modes. Right: Resulting multi-mode laser modes with relative intensities. Note: mode $\lambda$ 's are not linearly spaced (approx linear for large $m$ ), but mode freq's are linear. See formula in background section. . . . . . . . . . . . .

3.12 Multi-mode REM current pulse simulation, $N$ starting at $N_{t r}$ for 3 -mode simulation. $N$ and $S$ have units $\mathrm{cm}^{-3}$ while phase is in radians and chirp rad/s. 38

3.13 Multi-mode REM current pulse simulation, $N$ starting at $N_{t r}$ for 11-mode simulation. $N$ and $S$ have units $\mathrm{cm}^{-3}$ while phase is in radians and chirp rad/s. 39

3.14 Multi-mode REM current pulse simulation, showing $E$ magnitude for 3-mode simulation. . . . . . . . . . . . . . . . . . . 39 
3.15 Multi-mode REM current pulse simulation, showing $E$ magnitude for 31-mode simulations. . . . . . . . . . . . . . . . . .

3.16 Multi-mode REM current pulse simulation, showing zoom of $E$ magnitude for 11 mode simulation without a random phase. Pulses grow and get extremely narrow as number of modes increase. . . . . . . . . . . . . . . .

3.17 Multi-mode REM current pulse simulation, showing $E$ magnitude for 31 mode simulation starting each mode with a random phase. Bottom is a zoom in of top.

3.18 TLM model block diagram showing circuit elements. . . . . . . . . . . . . . 43

3.19 Active layer showing discretization of $z$ (along length) . . . . . . . . . . . . 45

4.1 Simple $R C$ circuit. Resistors shown as conductances. Nodes numbered within circles. . . . . . . . . . . . . . . . . . . . 47

4.2 Resistor with labeled nodes and corresponding 'stamp'. . . . . . . . . . . . . 47

4.3 Resistor with labeled nodes and corresponding 'stamp' when one node is ref-

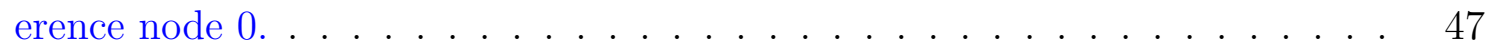

4.4 Resistor connected to current source. Resistor shown as conductance. Node numbered within circle. . . . . . . . . . . . . . . . . . 48

4.5 Resistors connected in series to current source. Resistors shown as conductances. Nodes numbered within circles. . . . . . . . . . . . . . . .

4.6 Current source with labeled nodes and corresponding 'stamp'. Note that this stamp is applied to the source vector $\boldsymbol{b}$. If one of the nodes is the reference node leave it empty. . . . . . . . . . . . . . . . . . . . . . . . . . 49

4.7 Resistor connected in series to voltage source. . . . . . . . . . . . . . . . . 49

4.8 Voltage source with labeled nodes and corresponding stamp. Note that this stamp is applied to both the $\boldsymbol{G}$ matrix and the source vector $\boldsymbol{b}$. A new column and row are added representing the source current. If one of the nodes is the reference node leave it empty. . . . . . . . . . . . . . . . . .

4.9 Capacitor with labeled nodes and corresponding 'stamp'. If one of the nodes is the reference node leave its rows/columns empty. . . . . . . . . . . . 50

4.10 Simple $R C$ filter circuit. . . . . . . . . . . . . . . . . . . . . . . . . 51

4.11 Inductor with labeled nodes and corresponding stamp. A new column and row are added representing the inductor current. If one of the nodes is the reference node leave it empty. . . . . . . . . . . . . . . . . . . . . . 52

4.12 Example nonlinear circuit. . . . . . . . . . . . . . . . . . . . 53

4.13 Nonlinear resistor with labeled nodes and corresponding stamp. Note that this stamp is applied to the nonlinear $\boldsymbol{f}$ vector. If one of the nodes is the reference node leave it empty. . . . . . . . . . . . . . . . . . . . 54

4.14 Example nonlinear capacitor circuit. . . . . . . . . . . . . . . . . 54

4.15 Nonlinear capacitor with labeled nodes and corresponding stamp. Note that this stamp is applied to the $\boldsymbol{G}$ and $\boldsymbol{C}$ matrices as well as the nonlinear $\boldsymbol{f}$ vector. If one of the nodes is the reference node leave it empty. . . . . . . . . 
5.1 Traveling Wave propagation with zero $\beta$ : no gain and output at same frequency with delay. . . . . . . . . . . . . . . . . .

5.2 Traveling wave propagation with real $\beta$ : no gain and no frequency shift, but a spatial frequency shift. . . . . . . . . . . . . . . . . . .

5.3 Traveling Wave propagation with real $\beta$ using sinusoidal input: no gain and no frequency shift. Show in 3D? . . . . . . . . . . . . . . . 65

5.4 Traveling Wave propagation with imaginary $\beta$ : Gain with no frequency shift. 67

5.5 Traveling Wave propagation with imaginary $\beta$ using sinusoidal input: Gain with no frequency shift. . . . . . . . . . . . . . . . . . 68

5.6 Plot of $f(x)$ showing the forward difference - the derivative at $x_{i}$ calculated from the value at the same $x_{i}$ and the value forward of it $x_{i+1} \ldots \ldots$. . . . 71

5.7 Plot of $f(x)$ showing the backward difference - the derivative at $x_{i}$ calculated from the value at the same $x_{i}$ and the value backward from it $x_{i-1}$. . . . .

5.8 TWM Gaussian pulse propagation. $\gamma_{p}=2 \pi\left(2 \times 10^{11}\right)[\mathrm{rad} / \mathrm{s}]$. Note that if we observed the pulse propagate through the waveguide we would see it shrinking and widening. . . . . . . . . . . . . . . . . . 77

5.9 TWM rectangular pulse propagation. Note the phase is always 0 only because $\omega_{0}=0$ which means the Lorentzian has no imaginary term. . . . . . . . . 78

5.10 TWM resonant cavity modes determine $k$ and hence $\lambda \ldots \ldots$. . . . . . . 80

6.1 Optical signal (const amplitude) propagating through traveling wave laser model. Right reflector has been removed. Starting from transparency the current step supplies carriers until the stimulated emission is sufficient to establish an equilibrium. a) Carrier density. b) Photon density. c) Current step. . . . . . . . . . . . . . . . . . . . .

6.2 Carrier distribution of optical signal propagating through traveling wave laser model. Carrier concentration in $\mathrm{cm}^{-3}$ versus laser segment number $(z)$. . . .

6.3 Comparison of traveling wave models: Without $N$ and integrated with $N$. . .

6.4 TWM carrier distribution . . . . . . . . . . . . . . . . . 86

6.5 TWM carrier distribution after the right-hand mirror has been removed. . . 87

7.1 REM single-mode and TWM laser models turn on during current pulse showing carrier and photon densities and the current pulse. . . . . . . . . . . .

7.2 REM single-mode and TWM laser models turn on showing E-field and FFT after laser models turn on. . . . . . . . . . . . . . . . . . . .

7.3 REM multi-mode with 11 modes and TWM laser models as they turn on with a current pulse. . . . . . . . . . . . . . . . .

7.4 REM multi-mode with 11 modes and TWM laser models showing FFT after laser models turn on. . . . . . . . . . . . . . . . . . . . . .

7.5 E-field, phase and chirp for REM 11-mode simulation with initial phase of zero for each mode. . . . . . . . . . . . . . . . . . 
7.6 E-field, phase and chirp for two runs of the same 11-mode REM simulation. Each run starts with a random initial phase on each mode. This first plot shows one random initial phase while the second plot shows five runs each with different random initial phases for each mode. . . . . . . . . . . . .

7.7 E-field, phase and chirp for single-mode run of REM starting without random phase (top) and starting with random phase (bottom). . . . . . . . . .

7.8 E-field, phase and chirp for two runs of single-mode REM compared with TWM. Henry factor set to 0 and $N$ starting at transparency. . . . . . . . . .

7.9 Comparison of REM simulation with Henry factor of 0 and 5. Carrier density $N$, Photon density, phase and $E$-field. A zoom in on the $E$-field for the second mode is shown in bottom plot where the frequency shift is apparent. . . . .

7.10 Carrier density $N$ and photon density with Henry factor of 0 and 5 for 3-mode REM compared with TWM.

7.11 E-field, phase and chirp for two runs of single-mode REM compared with TWM. Henry factor set to 5 and $N$ starting at transparency. . . . . . . . . .

$7.12 E$-field, phase and chirp for two runs of 3-mode REM compared with TWM. Henry factor set to 0 and $N$ starting at transparency. . . . . . . . . . . . 100

7.13 E-field, phase and chirp for two runs of 3-mode REM compared with TWM. Henry factor set to 5 and $N$ starting at transparency. . . . . . . . . . .

7.14 Comparison of simulation with Henry factor of 0 and 5 for single-mode REM and TWM showing carrier density $N$ (starting at threshold) and photon density. The plots are almost identical for the 3-mode REM. Note the current is a step. . . . . . . . . . . . . . . . . . . .

$7.15 E$-field, phase and chirp for three runs of single-mode REM compared with TWM. Henry factor set to 0 and $N$ starting at threshold. . . . . . . . . . .

7.16 E-field, phase and chirp for single-mode run of REM and TWM with Henry factor of 5 starting at threshold. . . . . . . . . . . . . . . . . .

7.17 E-field, phase and chirp for three runs of 3-mode REM compared with TWM. Henry factor set to 0 and $N$ starting at threshold. Note that the phase had to be filtered. . . . . . . . . . . . . . . . . . . .

7.18 E-field, phase and chirp for three runs of 3-mode REM compared with TWM. Henry factor set to 5 and $N$ starting at threshold. . . . . . . . . . . .

7.19 E-field, phase and chirp for three runs of single-mode REM compared with TWM. Henry factor set to 0 and $N$ starting at threshold. First run has $\delta=190,2$ nd $\delta=196.5,3$ rd $\delta=200 \ldots \ldots \ldots \ldots$

$7.20 \mathrm{E}$-field, phase and chirp for three runs of single-mode REM compared with TWM. Henry factor set to 5 and $N$ starting at threshold. First run has $\delta=190,2$ nd $\delta=196.5,3$ rd $\delta=200$. Note: TWM not affected by REM modes. 109

7.21 Computation time for TWM and various numbers of modes for the multimode REM. . . . . . . . . . . . . . . . . . . . . . . 111

7.22 Comparing 41-mode REM and 21-segment TWM E-field and spectrum. The number of modes required to match the number of segments is about double. 
7.23 Comparing 51-mode REM and 51-segment TWM E-field and spectrum. The plot shows that the number of modes required to match the number of segments is about equal. . . . . . . . . . . . . . . . .

A.1 Delay of carrier modulated with a square pulse through waveguide without gain or loss. Top shows physical extent. Bottom 2 show signals versus time. 2nd shows input at left side $(z=0)$. Bottom shows output after a delay of $\lambda\left(z=z_{1}\right) \ldots \ldots \ldots \ldots \ldots \ldots \ldots$

A.2 Delay of carrier modulated with a square pulse through waveguide without gain or loss. Top shows physical extent. Bottom 3 show signals in time. 2nd shows input at left side $(z=0)$. 3rd shows input after a delay of $\frac{\lambda}{2}\left(z=z_{1}\right)$. Bottom shows output at right side after a delay of $\lambda\left(z=z_{2}\right) . \ldots . . .123$

A.3 Phase factor due to difference in carrier frequencies. . . . . . . . . . . . 126

B.1 Complex exponential $e^{-i \beta z} e^{i\left(k_{e} z-\omega_{e} t\right)}$ rotating CW. Bottom 3-D plot shows complex value over $z$ : Left/right axis is $z$, other two are real/imaginary. . . .

B.2 Complex exponential $e^{-i \beta z} e^{-i\left(k_{e} z-\omega_{e} t\right)}$ rotating CCW. Bottom 3-D plot shows complex value over $z$ : Left/right axis is $z$, other two are real/imaginary. . . .

B.3 Sum of complex exponentials: rotating cosine. Bottom 3-D plot shows complex value over $z$ : Left/right axis is $z$, other two are real/imaginary. . . . . .

C.1 'Vector' plots of the complex envelope over time of individual modes and their sum for the 3-mode REM. Top: modes 2 and 3. Bottom: fundamental and sum. These are 3-D plots showing complex value over $z$ : Left/right axis is $z$, other two are real/imaginary. . . . . . . . . . . . . . .

C.2 Top: $E$-field as a sum of simple sinusoids with random initial phases. Bottom: Phase of E-field. . . . . . . . . . . . . . . . . . . . . . . . . . . . . . 135

C.3 E-field phase as a sum of phasors for a 5-mode REM. The phasors come in pairs which rotate in opposite directions. . . . . . . . . . .

C.4 E-field phase of a 5-mode REM showing phase accumulating with mode 4.Left: simple simulation. Right: MNA multi-mode REM simulation. . . . . . . . .

C.5 E-field phase as a sum of simple sinusoids for a 5-mode REM. Each run has the same initial and final amplitude configuration but with different initial phases. In the middle a typical mode distribution is used. . . . . . . . . . .

C.6 E-field phase as a sum of simple sinusoids for a 5-mode REM. Each run has the same initial and final amplitude configuration but with different initial phases. In the middle a typical mode distribution is used. . . . . . . . . . . .

C.7 E-field phase for a 5-mode REM. Left: random initial phases. Right: random phases but symmetrical. . . . . . . . . . . . . . . . . . . 140

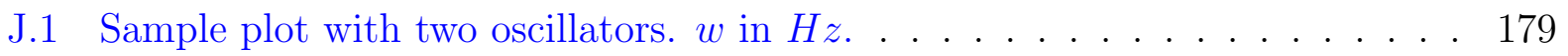




\section{Glossary}

CB Conduction Band. vii, 13

DBR Distributed Bragg Reflector. 82

DC Direct Current. 2, 44, 51, 55

DE Differential Equation. viii, 4, 23, 27, 28, 30-33, 53, 69, 71-73, 76, 81-83, 114, 152

DFB Distributed Feedback Laser. 79, 82, 113

DOS Density of States. vii, 13

FDM Finite Difference Method. 69, 73

FDTD Finite Difference Time Domain. 53, 57, 69, 71, 73, 76

HE Hybrid Mode. 6

IC Integrated Circuit. vii, 1, 12, 13

KCL Kirchoff's Current Law. 46-49, 53, 54

LED Light Emitting Diode. viii, 12, 18, 19

MNA Modified Nodal Analysis. xii, 2, 19, 23, 44, 46, 53, 56, 57, 69, 72, 81, 83, 110, 114, $115,134,135,137$

OptiSPICE Commercial Optical SPICE simulator. 56, 115

PDE Partial Differential Equation. 59, 60, 62, 63, 66

REM Rate Equation Model. i, viii-xii, 1, 2, 18, 19, 23, 27, 29-34, 36-45, 56, 57, 76, 81-83, 88-115, 133-140, 142, 161, 162, 164, 167

SOA Semiconductor Optical Amplifier. 59, 115 
SPICE Simulation Program with Integrated Circuit Emphasis. i, 1, 23, 46, 53, 56, 114, 115

SVEA Slowly Varying Envelope Approximation. 44, 58, 121, 158, 160, 165

TE Transverse Electric Mode. vii, 6-8

TEM Transverse Electromagnetic Mode. 6

TLM Transmission Line Model. i, ix, 2, 19, 23, 43, 44, 57, 114

TM Transverse Magnetic Mode. 6

TWM Traveling Wave Model. i, x-xii, 1, 2, 19, 23, 25, 44-46, 57-60, 69, 76-84, 86-92, 94-115, 126, 128, 164, 167

VB Valence Band. vii, 13

VCSEL Vertical-Cavity Surface-Emitting Laser. 82 


\section{Chapter 1}

\section{Introduction}

\section{$1.1 \quad$ Laser Models}

Up to this point in time, semiconductor laser models have been created for investigating a particular laser and limited to a specific aspect of operation such as field intensity, power or thermal for example. The bulk of the design is done by experience and adjusted by trial and error. Models are fitted to the specific laser by measuring parameters of the laser. The design proof is done by building the laser. This is similar to when Integrated Circuit (IC) designs were tested by breadboarding the design before the expensive fabrication process was done. For ICs design verification by simulation is now the norm. This will soon be the same for optical circuits, and specifically, lasers. The laser specific models will give way to more generic models which will need to be integrated with the electronic simulators. This creates a need for a compact SPICE-like model. [1] This thesis looks at current laser models and compares them to the TWM as a more detailed model that can potentially meet the goals of the future optoelectronic simulators.

\subsection{Thesis Motivation}

The classic REM models currently used give the overall dynamics of a laser but are found to be lacking in specific functionality. Enhancements have been made to mitigate this but of course this increases the computational cost of them. As we demand increased accuracy more parameters and functionality need to be added to the otherwise simple model. The TWM model is based on a more physical design that uses Maxwell's equations with some simplifications and approximations allowing us to predict the behaviour to a potentially higher degree of accuracy. Being a compact model it is still much simpler than a fullwave electromagnetic simulation but nonetheless comes with extra computational cost - the question is, is it worth it? Can the laser dynamics be predicted by calculating the optical fields in a more realistic physical way? As lasers become more and more widespread the need to integrate them into commercial circuit simulators grows. Since more and more circuits 
use them they need to have a better design process without resorting to actual fabrication and test.

\subsection{Contributions}

Although not part of this thesis this research contributed to the following papers:

- DC operating point paper [2].

- MNA Engine integration paper [3].

At the time of writing these papers had not been published.

\subsection{Organization}

This thesis is organized in the following manner. Chapter 2 presents the background on topics of laser physics at a basic level so that the models discussed can be understood. This is focussed mainly on the physical devices, how they operate and their properties.

Chapter 3 gives an overview of the laser models currently being used by the design community. Some properties of the complex envelope are described in some detail so that the models' usage is better understood. The classic REM is described along with the enhancements it has undergone since conception. The TLM is briefly looked at and then the TWM fundamentals are described.

Chapter 4 gives an overview of how these models are used in a commercial MNA engine and how that engine works.

Chapter 5 looks at the properties and dynamics of the TWM in detail. It starts with the numerical method used by the model and then looks at different aspects of the model.

Chapter 6 describes how the TWM is modified to form the Traveling Wave Laser Model and how it behaves.

Chapter 7 compares the REM and TWM laser models to see the benefits and drawbacks of each.

Chapter 8 summarizes the findings and makes recommendations on how well the TWM meets the needs of future commercial simulators and what still needs to be overcome or investigated.

The appendixes provide detailed calculations and examples that may be of interest to fellow researchers but are not essential to understanding the research claims. 


\section{Chapter 2}

\section{Laser Operation}

\subsection{Maxwell's Equations}

There are different forms of Maxwell's equations depending on the type of media and assumptions about it, as well as what the equations are being used for. In our case we will give the equations in differential form assuming no external sources. This greatly simplifies them. These equations can be found in any electromagnetic theory physics text such as [4].

$$
\begin{aligned}
\nabla \cdot \boldsymbol{D} & =\rho \\
\nabla \cdot \boldsymbol{B} & =0 \\
\nabla \times \boldsymbol{E} & =-\frac{\partial \boldsymbol{B}}{\partial t} \\
\nabla \times \boldsymbol{H} & =\boldsymbol{J}+\frac{\partial \boldsymbol{D}}{\partial t}
\end{aligned}
$$

We assume a linear, homogeneous, and isotropic medium with the following parameters: $\sigma$, $\varepsilon$, and $\mu$ and has the constitutive relations for time varying fields

$$
\begin{array}{r}
\boldsymbol{D}=\varepsilon \boldsymbol{E}=\varepsilon_{0} \boldsymbol{E}+\boldsymbol{P} \\
\boldsymbol{B}=\mu \boldsymbol{H}=\mu_{0}(\boldsymbol{H}+\boldsymbol{M}) \\
\boldsymbol{J}=\sigma \boldsymbol{E}+\rho \boldsymbol{u}
\end{array}
$$

\subsubsection{Phasor Form}

A phasor is a complex number that contains the amplitude and phase of a sinusoidal oscillation. For example given a sinusoidal current $I(t)=I_{0} \cos (\omega t+\phi)$ the phasor would be $\tilde{\boldsymbol{I}}=I_{0} e^{i \phi}$. To get the time-varying field we take the real part of a complex exponential varying with time

$$
I(t)=\Re\left[\tilde{\boldsymbol{I}} e^{i \omega t}\right]
$$


This is the same for vector time-harmonic fields

$$
\boldsymbol{A}=\Re\left[\tilde{\boldsymbol{A}} e^{i \omega t}\right]
$$

and then we note that the derivative

$$
\frac{\boldsymbol{d} \boldsymbol{A}}{d t}=\Re\left[i \omega \tilde{\boldsymbol{A}} e^{i \omega t}\right]
$$

This will give us Maxwell's equations in phasor form (time factor is supressed) as

$$
\begin{aligned}
\nabla \cdot \tilde{\boldsymbol{D}} & =\rho \\
\nabla \cdot \tilde{\boldsymbol{B}} & =0 \\
\nabla \times \tilde{\boldsymbol{E}} & =-i \omega \tilde{\boldsymbol{B}} \\
\nabla \times \tilde{\boldsymbol{H}} & =\tilde{\boldsymbol{J}}+i \omega \tilde{\boldsymbol{D}}
\end{aligned}
$$

\subsubsection{Wave Propagation in Dielectrics}

We consider a linear, homogeneous, and isotropic medium that is charge free (macroscopic $\rho=0$ ) which then yields Maxwell's equations in phasor form as

$$
\begin{aligned}
\nabla \cdot \tilde{\boldsymbol{D}} & =0 \\
\nabla \cdot \tilde{\boldsymbol{B}} & =0 \\
\nabla \times \tilde{\boldsymbol{E}} & =-i \omega \tilde{\boldsymbol{B}} \\
\nabla \times \tilde{\boldsymbol{H}} & =(\sigma+i \omega \varepsilon) \tilde{\boldsymbol{E}}
\end{aligned}
$$

where (2.6) and (2.8) have been used. These can be rearranged to give

$$
\begin{aligned}
\nabla^{2} \tilde{\boldsymbol{E}}-\gamma^{2} \tilde{\boldsymbol{E}} & =0 \\
\nabla^{2} \tilde{\boldsymbol{H}}-\gamma^{2} \tilde{\boldsymbol{H}} & =0
\end{aligned}
$$

which are the homogeneous vector Helmholtz equations where $\gamma=\alpha+i \beta$, a complex number.

If we assume the wave propagates along the $z$ direction and $\tilde{\boldsymbol{E}}$ has only an $x$ component then these reduce to a scalar wave equation which is a linear homogeneous DE with a known solution. Once the boundary conditions are used and the time factor put back in we get

$$
\boldsymbol{E}(z, t)=E_{0} e^{-\alpha z} \cos (\omega t-\beta z) \hat{x}
$$

$\boldsymbol{H}(z, t)$ can be gotten by either solving in a similar manner for $\boldsymbol{E}(z, t)$ or by using Maxwell's equations to generate $\boldsymbol{H}(z, t)$ from $\boldsymbol{E}(z, t)$ giving

$$
\boldsymbol{H}(z, t)=\Re\left[H_{0} e^{-\alpha z} e^{i(\omega t-\beta z)} \hat{y}\right]
$$


where $H_{0}=\frac{E_{0}}{\eta}$ and $\eta$ is the intrinsic impedance. Since $\boldsymbol{H}(z, t)$ can always be obtained from $\boldsymbol{E}(z, t)$ our focus is on the latter. A plot of $\boldsymbol{E}(z, t)$ is shown in figure 2.1. The field attenuates by the factor $e^{-\alpha z}$ and so $\alpha$ is called the attenuation factor. Note that these fields are perpendicular to the direction of travel and so are called transverse. Of course as Maxwell's equations dictate $\boldsymbol{E}$ is perpendicular to $\boldsymbol{H}$.

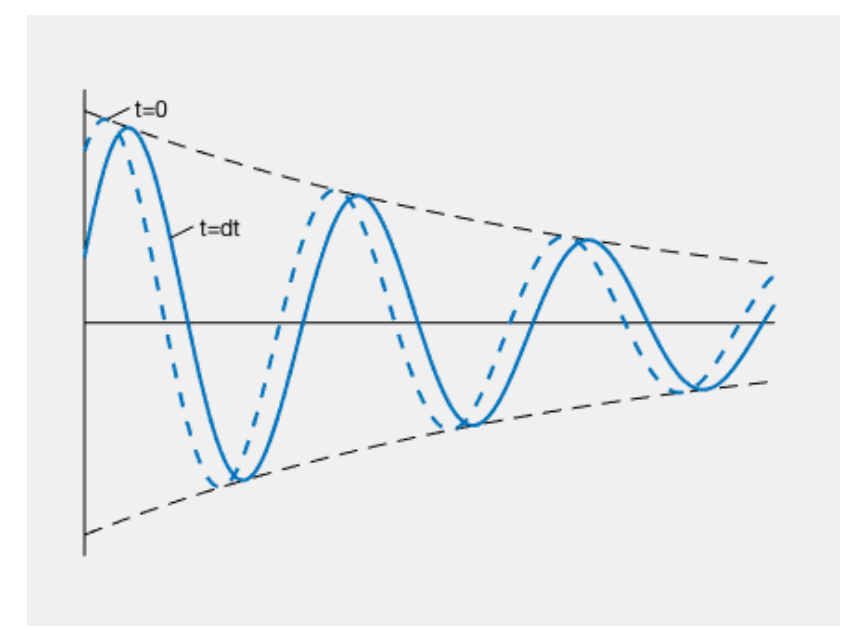

Figure 2.1: Plot of $E$ field in a lossy medium traveling in $+z$ direction. Shown at two times: $t=0$ and $t=d t$. The field is attenuated as it penetrates the medium.

\subsubsection{Waveguide Modes}

We consider a rectangular waveguide as shown in figure 2.2. For now we take the simple case of perfectly conducting walls and a lossless dielectric material. From (2.22)) and (2.23)

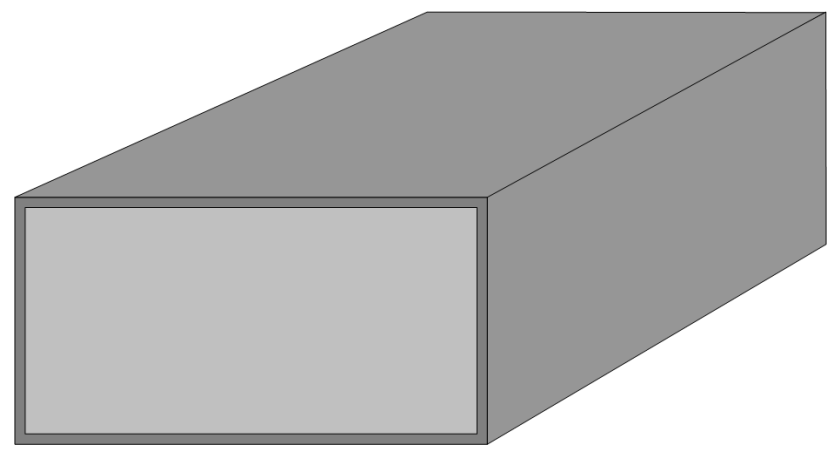

Figure 2.2: Rectangular waveguide filled with a homogeneous isotropic medium. Note that the length is infinite. 
we have

$$
\begin{aligned}
\nabla^{2} \tilde{\boldsymbol{E}}+k^{2} \tilde{\boldsymbol{E}} & =0 \\
\nabla^{2} \tilde{\boldsymbol{H}}+k^{2} \tilde{\boldsymbol{H}} & =0
\end{aligned}
$$

where $k=\omega \sqrt{\mu \varepsilon}$. $\gamma$ is changed to $k$ because the original equations were for the general case of a lossy dielectric and we now assumed the lossless case. These can be solved by separation of variables to give

$$
\begin{aligned}
& \tilde{E}_{z}(x, y, z)=\left(A_{1} \cos k_{x} x+A_{2} \sin k_{x} x\right)\left(A_{3} \cos k_{y} y+A_{4} \sin k_{y} y\right) e^{-\gamma z} \\
& \tilde{H}_{z}(x, y, z)=\left(B_{1} \cos k_{x} x+B_{2} \sin k_{x} x\right)\left(B_{3} \cos k_{y} y+B_{4} \sin k_{y} y\right) e^{-\gamma z}
\end{aligned}
$$

Instead of solving for the other four terms we use Maxwell's equations to solve for them in terms of the two we already know.

$$
\begin{aligned}
& \tilde{E}_{x}=-\frac{\gamma}{h^{2}} \frac{\partial \tilde{E}_{z}}{\partial x}-\frac{i \omega \mu}{h^{2}} \frac{\partial \tilde{H}_{z}}{\partial y} \\
& \tilde{E}_{y}=-\frac{\gamma}{h^{2}} \frac{\partial \tilde{E}_{z}}{\partial y}+\frac{i \omega \mu}{h^{2}} \frac{\partial \tilde{H}_{z}}{\partial x} \\
& \tilde{H}_{x}=\frac{i \omega \varepsilon}{h^{2}} \frac{\partial \tilde{E}_{z}}{\partial y}-\frac{\gamma}{h^{2}} \frac{\partial \tilde{H}_{z}}{\partial x} \\
& \tilde{H}_{y}=-\frac{i \omega \varepsilon}{h^{2}} \frac{\partial \tilde{E}_{z}}{\partial x}-\frac{\gamma}{h^{2}} \frac{\partial \tilde{H}_{z}}{\partial y}
\end{aligned}
$$

where

$$
h^{2}=\gamma^{2}+k^{2}=k_{x}^{2}+k_{y}^{2}
$$

From these equations we see that the field solutions come in four categories:

1. Transverse Electromagnetic Mode (TEM): $\tilde{E}_{z}=0=\tilde{H}_{z}$ so both $\boldsymbol{E}$ and $\boldsymbol{H}$ fields are transverse to the direction of wave propagation.

2. Transverse Electric Mode $(\mathrm{TE}): \tilde{E}_{z}=0, \tilde{H}_{z} \neq 0$ so both $\tilde{\boldsymbol{E}}_{x}$ and $\tilde{\boldsymbol{E}}_{y}$ of the electric field are transverse to the direction of wave propagation.

3. Transverse Magnetic Mode (TM): $\tilde{E}_{z} \neq 0, \tilde{H}_{z}=0$ so both $\tilde{\boldsymbol{H}}_{x}$ and $\tilde{\boldsymbol{H}}_{y}$ of the magnetic field are transverse to the direction of wave propagation.

4. Hybrid Mode $(\mathrm{HE}): \tilde{E}_{z} \neq 0, \tilde{H}_{z} \neq 0$ so neither $\tilde{\boldsymbol{E}}$ or $\tilde{\boldsymbol{H}}$ fields are transverse to the direction of wave propagation.

For the rectangular waveguide if both $\tilde{E}_{z}=0$ and $\tilde{H}_{z}=0$ then all field components vanish. Therefore a rectangular waveguide cannot support TEM mode. 


\subsubsection{Ridge Waveguide}

The rectangular waveguide infinite in length with perfectly conducting walls is good for understanding basic field propagation but our model needs to match real laser diodes better. Many texts [5] have a development on how to go beyond the rectangular waveguide with perfectly conducting walls. To this end we turn to a ridge waveguide which more closely matches our laser diode with dielectric layers of finite dimensions. [6] A typical semiconductor laser structure shown in figure 2.3. This structure has a finite width and height but for now could be analyzed as still infinite in length. In most lasers the width is greater than a very thin height. Above, below and beside the active layer the different index of refraction acts similar to the perfectly reflecting walls of the rectangular waveguide. If we treat the length as infinite, the transverse modes will be constant down the length of the waveguide. For

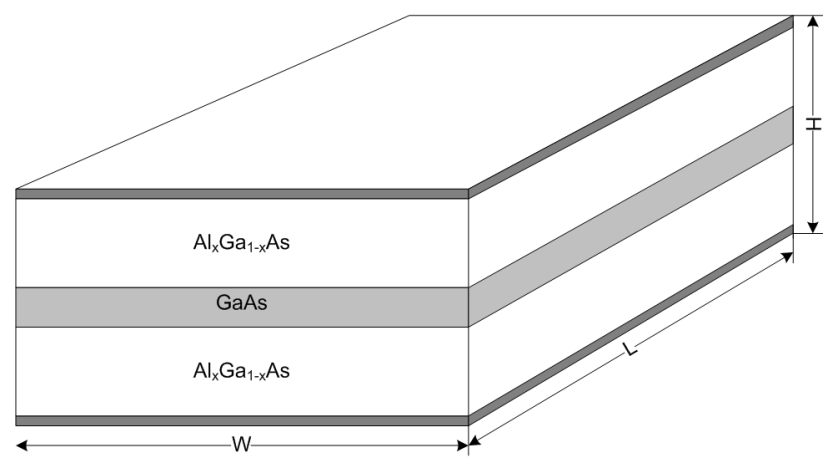

Figure 2.3: Laser diode heterojunction structure. Top and bottom layers are metal contacts connected to a voltage source. With infinite length and width this is a modeled as a slab waveguide but with a finite width becomes a ridge waveguide.

analysis we start with a slab waveguide which has a finite height but is infinite in width and length. Using Maxwell's equations we can solve this problem and find that the transverse modes are similar to the rectangular waveguide with perfectly conducting walls. For TE polarization the electric field only has a component in the vertical direction and we see the transverse modes in figure 2.4. The difference to the rectangular waveguide with perfectly conducting walls is that the field 'leaks' into the outer dielectric and decays. The amount of 'leakage' determines the confinement factor (explained further in section 2.3.4).

The next step is to have asymmetrical dielectrics above and below the slab waveguide. What we find is that the rate of decay will be faster or slower as the permitivitty (and so index of refraction) varies. This will be the case with a laser diode as the substrate will have a different permitivitty than the material above the diode or ridge waveguide. Note that this will also affect the cutoff frequency (lowest frequency a mode will propagate) of the waveguide.

Finally we turn to rectangular dielectric waveguides which are finite in both height and width like the rectangular waveguide with perfectly reflecting walls but have dielectric materials for walls. We will assume the width is greater than the height as is usually the case 

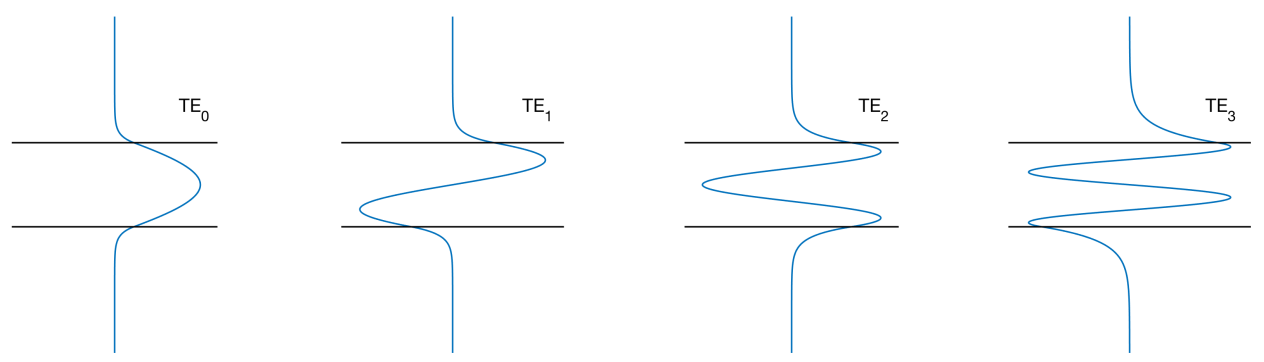

Figure 2.4: TE modes of symmetrical ridge waveguide.

in actual devices. Figure 2.5 shows a cross-section of a rectangular dielectric waveguide. In

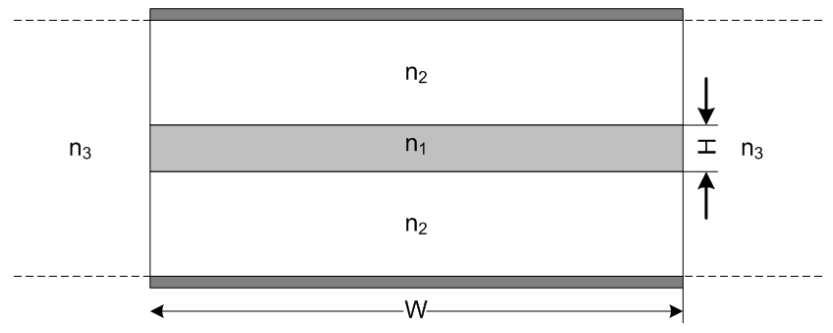

Figure 2.5: Cross-section of a rectangular dielectric waveguide. All index regions could be different and we could ignore the metal at the top and bottom for a general case but it will likely be of this form.

this case the solutions to Maxwell's equations will be the hybrid modes because the electric field is never zero in one of the transverse directions. They are close to zero and so again are similar to the fields in the perfect conductor case shown in figure 2.6.

The ridge waveguide is a good approximation to a semiconductor laser diode. In figure 2.7 we have cut away the material above the waveguide. Note that it is the finite width of the 'ridge' or top layer that changes the effective index on either side of it. This is what confines the field in the lateral direction. This makes it similar to the rectangular waveguide with perfectly conducting walls which is easy to analyze.

We will use this as a basis for understanding the modes in the laser diode.

\subsubsection{Lossy Waveguide}

Remember that the rectangular waveguide in section 2.1.3 was an ideal case with perfectly conducting sides and a lossless dielectric core. In a real material the dielectric will be lossy. If we refer back to section 2.1.2 we see that the main difference between a lossless and lossy dielectric is the attenuation of the wave as it penetrates the dielectric. So our waveguide modes will be the same - they will just attenuate as they propagate down the rectangular waveguide. 


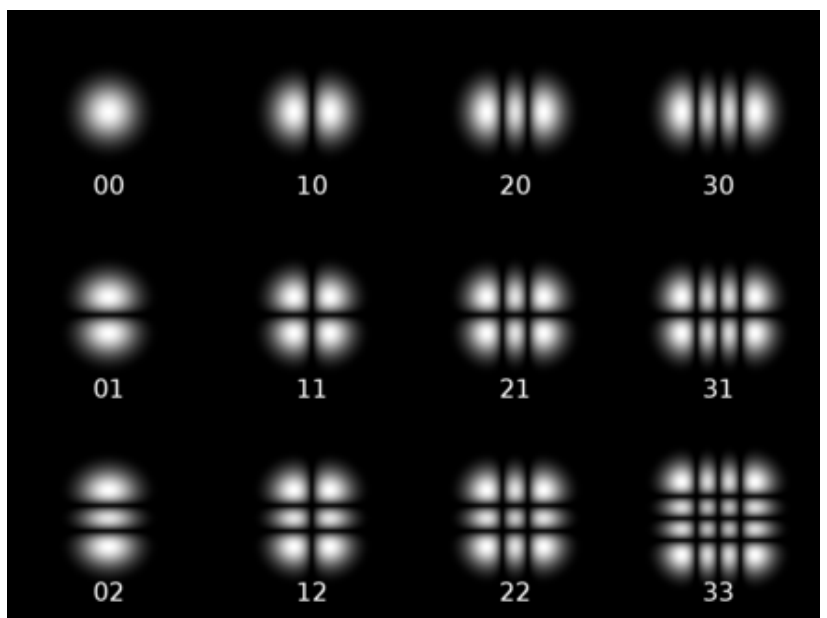

Figure 2.6: Cross-sectional view of transverse field patterns and intensities for rectangular dielectric waveguide.

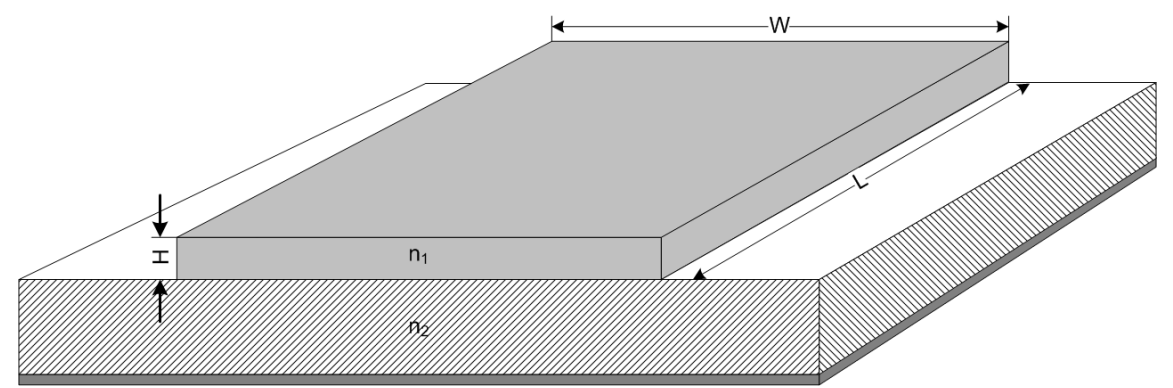

Figure 2.7: Ridge waveguide approximated by a rectangular dielectric waveguide. The dielectric material above the waveguide for the laser active layer has been removed.

\subsubsection{Longitudinal Modes}

In a laser - or any real waveguide - the length will never be infinite. This means that we should redo the analysis for a cavity with Maxwell's equations. This is not necessary because the length of a laser cavity is usually much longer than the height and width so the rectangular waveguide is still a good approximation for the transverse modes. Taking account of the finite length will create longitudinal modes. With perfect conducting ends we can see that just as in the $x$ and $y$ directions we are permitted integral half-wavelengths from the sinusoidal fields being forced to 0 at the walls.

$$
L=\frac{m \lambda}{2}
$$




\subsection{Elements of a Laser}

Laser operation requires three basic elements: pumping to produce a population inversion, gain with stimulated emission, and optical feedback usually with mirrors.

\subsubsection{Pump}

We consider the simplest form of a laser that has atoms at two energy levels, $E_{1}$, and $E_{2}$ with $E_{2}$ being higher $\left(E_{2}>E_{1}\right)$. These levels are at an appropriate energy such that light of some frequency can be absorbed or emitted. This frequency is given by

$$
\omega=\frac{E_{2}-E_{1}}{\hbar}
$$

When a photon of this frequency is incident on the atom and is absorbed, the atom's energy increases from $E_{1}$ to $E_{2}$. As the system will tend to a state of lower energy, at some point the atom will relax down from $E_{2}$ to $E_{1}$ and a photon of the same frequency will be emitted. The term population refers to the number of atoms at each energy level; in this case $N_{1}$ and $N_{2}$. Under normal conditions, when the atoms are at a given temperature, most will drop to the lower energy so $N_{1} \gg N_{2}$. Because of thermal energy $N_{2}$ will never be zero but will be much smaller than $N_{1}$. The situation where $N_{2}>N_{1}$ is called a population inversion because it is not the normal state of the atoms. The adding of energy is known as 'pumping' and is the first required element of a laser. 'Pumping' of a laser can be acheived many ways in different kinds of lasers. We will look at semiconductor lasers. In semiconductor lasers the 'pumping' is acheived by injecting a current through the laser diode.

\subsubsection{Gain Medium}

The second required element of a laser is an optical gain medium. This simply means that if light were incident on the medium the beam would grow stronger as it propagates through. This is not the typical behavior of light in a medium. In glass, for example, the light intensity is invariable attenuated as the light is absorbed.

To see how optical gain is possible let us review the emission and absorption of light in an atom. Absorption as mentioned above is simply when an incident photon is absorbed and the energy of the atom is raised by the energy of the photon. This requires that the energy of the photon match the energy difference between the two energy levels of the atom. See figure 2.8 left. When the atom returns to the lower energy level at some random time releasing a photon of the same energy this is called spontaneous emission. See figure 2.8 middle. It is important to note that the probability of this happening is very high as the lower energy level is the preferred state. There is a third transition that occurs when the atom is in the higher energy state and is hit by a photon of the energy difference between the states. There is a probability that the atom will transition down to the lower energy state releasing a photon without absorbing the incident photon. The critical thing to note 
is that the emitted photon will be in exact phase with the incident photon. This is called stimulated emission and is the key to optical gain in a medium. See Figure 2.8 right.

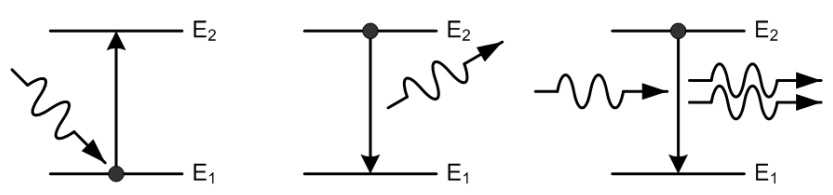

Figure 2.8: Left: Absorption. Middle: Spontaneous emission. Right: Stimulated emission.

If the medium has been 'pumped' so that there is a population inversion then the probability of stimulated emission becomes very high. At first, with no incident photons, the higher energy atoms will release photons with energy equal to the difference between the two levels as they drop to the lower level. But this is of course the same energy photons that will create stimulated emission. Now each photon generated will stimulate other photons and these in-phase photons will cascade through the medium quickly increasing the number of coherent photons. See figure 2.9. This is the nature of the optical gain by stimulated emission and again is the second element required for a laser.

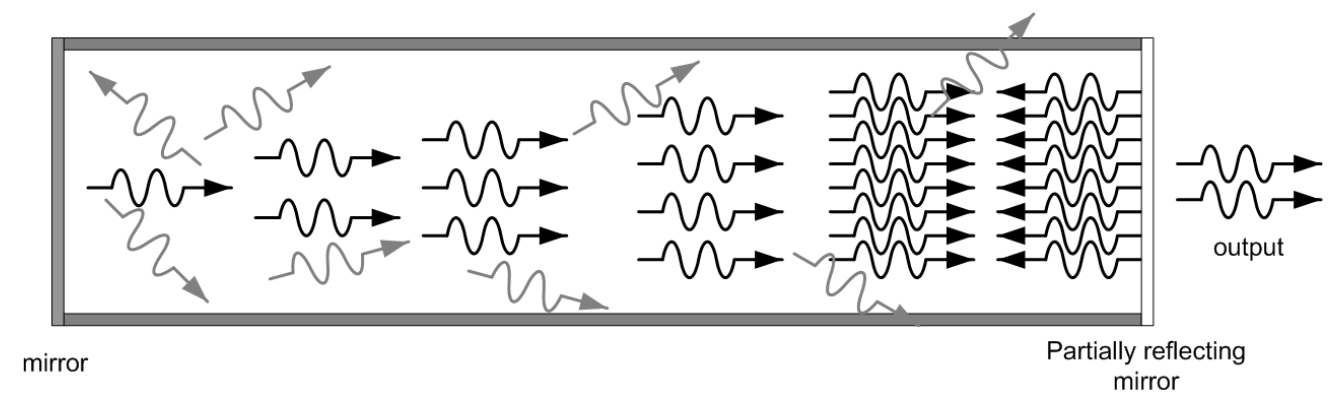

Figure 2.9: Cascade of initially spontaneous and then stimulated emission. There is always a small amount of spontaneous emission, but with a gain medium stimulated emission quickly takes over. Note that although the stimulated emission is shown growing in one direction it will build in both directions.

\subsubsection{Optical Feedback}

The last element required for a laser is optical feedback. With the first two elements we could build an optical amplifier because if the light were in the energy range for stimulated emission it would be amplified. But optical feedback (or mirrors) is required for lasing. Mirrors at both ends of the optical medium allow the cascading of stimulated emission to continue to amplify the light intensity each time the light passes through the medium. The photons may be emitted in all directions but they will only continue to grow in the path between the mirrors. This can create quite a strong beam of coherent light. Of course as the photons sweep through the medium they cause many atoms to drop to the lower state. 
If the pumping is not adequate to keep the population inversion maintained at a sufficient level only a pulse of light is produced because the higher energy level is depleted. As well there must be a way to tap off some of the light beam in order to use it so one of the mirrors must be a partial transmitter. The pumping must be enough to overcome all of the optical losses including portion lost at the output.

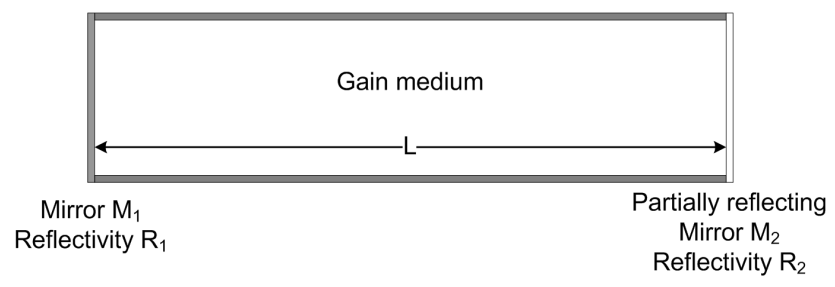

Figure 2.10: Physical structure of basic laser.

\subsection{Semiconductor Lasers}

We will be modeling semiconductor lasers. They are simple to build and are the most likely type of laser to be used with electronics and in particular integrated into an IC. This is our motivation to also integrate the optical circuit model with the electronic circuit simulator. We use the two-level model because it is the simplest way for understanding the basics of laser models.

Fabricating a semiconductor laser is pretty straightforward - it is essentially an LED with modifications. The energy levels are the valence band and the conduction band. Refer to 2.11. When a photon is absorbed an electron is raised into the conduction band (electron/hole generation). When the electron, with no stimulus, drops an energy level (electron/hole recombination), emitting a photon we have spontaneous emission. And when a photon causes electon/hole recombination emitting a second coherent photon we have stimulated emission. The 'pump' is provided by the diode current. The use of heterojunctions and band-gap engineering allows flexibility in choosing the frequency of the laser. This as well as the use of waveguides creates active regions where the carriers and photons are concentrated for a strong population inversion - the heterostructure confines the carriers (holes/electrons); waveguides confine the photons.

\subsubsection{Physical Structure}

The physical structure of a semiconductor laser is the same as an LED. An example of the physical structure is shown in figure 2.12. You can see that it is a diode with two ports (contacts) and an output facet for the light to escape. If the mirrors on the ends were removed it would be an LED. In the figure we show the diagram of a double-heterostructure configuration composed of $A l G a A s, G a A s$, and $A l G a A s$. This is a typical choice and because of the low doping in the center region the recombination due to impurities is low and efficiency 


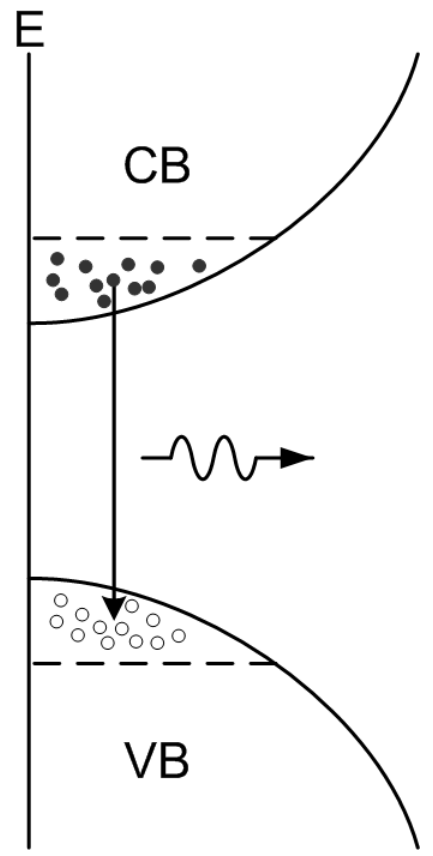

Figure 2.11: Semiconductor laser Density of States (DOS). The two energy levels of laser are the Conduction Band (CB) and the valence band (VB). The electron drops from CB to VB emitting a photon as spontaneous emission.

is high. This concentrates the carriers in the middle where they can be built up for interaction with the photons of stimulated emission.

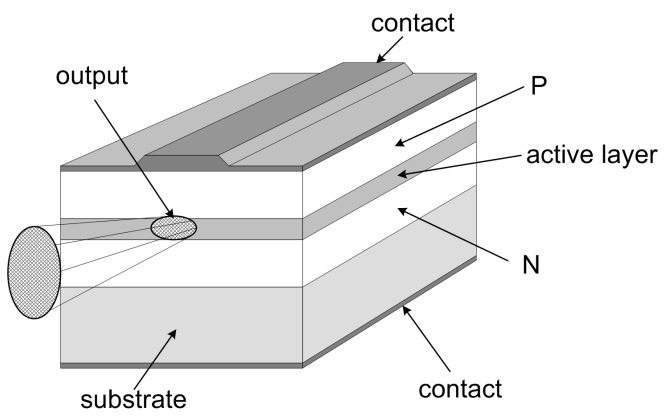

Figure 2.12: Physical structure of a slab semiconductor laser made on a single IC.

\subsubsection{Band Structure}

For a laser we would like to engineer the band structure so that we get efficient recombination of carriers to produce intense light. This is done best by direct bandgap materials such as GaAs because all of the energy is converted to light; none is lost to phonons or heat. A PN 


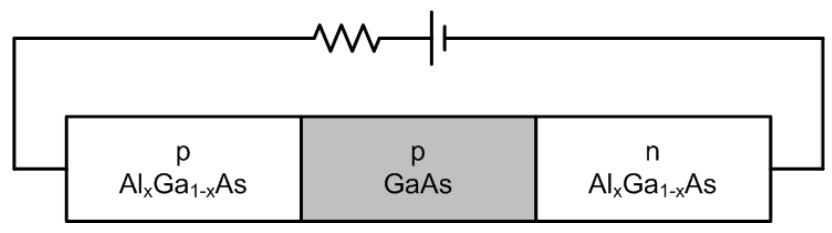

Figure 2.13: Semiconductor laser physical diagram.

junction diode would work but heterostructures are much more efficient. A heterostructure is a junction between two different bandgap materials. [7] A double-heterostructure has two junctions between materials with different bandgaps. A typical double-heterostructure band diagram is shown in figure 2.14. A double-heterostructure is better at confining the carriers so they are held longer with more time to recombine greatly increasing efficiency. It was this development in band engineering that allowed the use of semiconductor lasers at room temperature and facilitated the proliferation of them in many devices. The other advantage of the double-heterostructure is that it serendipitously causes photon confinement. This is explained in section 2.3.4. Because we can control the bandgap we can select our wavelength of the photons. This will not be a single wavelength but follows a Lorentzian shape as shown in figure 2.15 .

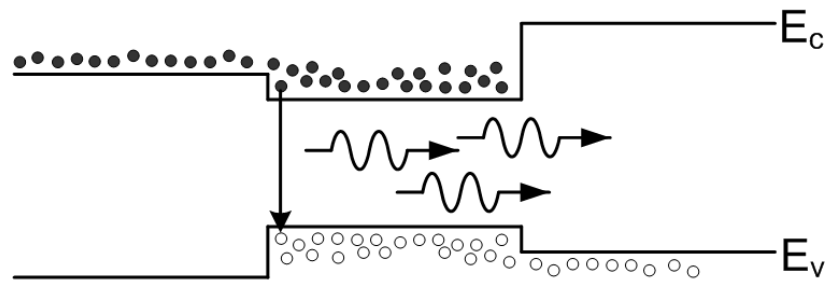

Figure 2.14: Typical semiconductor laser band structure. Carriers are confined by bandgap so they have lots of time to recombine emitting photons.

\subsubsection{Linewidth}

The optical spectrum of the light emitted from an energy transition is often modeled as Lorentzian. A first-order Lorentzian is given by

$$
\frac{\gamma_{p}}{\gamma_{p}+i\left(\omega-\omega_{0}\right)}
$$

where $\gamma_{p}$ will determine the width. A plot is shown in figure 2.15. For semiconductor lasers the line spectrum is not a Lorentzian. It has a tail and can be modeled with a sum of Lorentzians. For our purposes this is a detail that will concern us when we have a specific laser to model. 


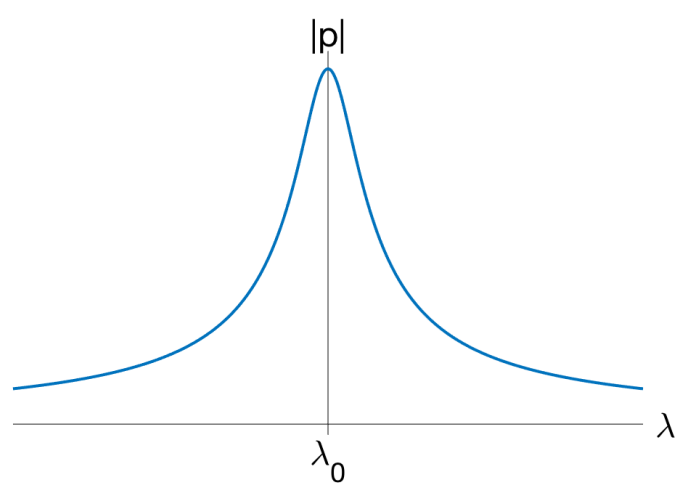

Figure 2.15: Semiconductor laser emission intensity modeled as a Lorentzian.

\subsubsection{Waveguide Structure}

The physical structure used to create the heterostructures also changes the index of refraction of the material. This fact allows the semiconductor to also be designed as a waveguide for photon confinement. From optics we know that if the outer indexes are smaller than that of the inner we will have total internal reflection and the light will be contained creating a waveguide. Fortunately this is possible while also allowing us to do bandgap engineering to create our heterostructures for carrier confinement. We will see that this is not perfect but creates a high concentration of carriers and photons which allows for a high level of interaction between them. Figure 2.16 shows the energy band diagrams of a forward-biased laser diode with a narrow active layer which has a higher index of refraction than the outer containing layers. The resulting optical intensity is shown in figure 2.17 and shows that although some of the energy leaks out the confinement is good. This is necessary when we want the stimulated emission to continue with as many carriers as possible to produce the highest gain medium possible which will give the strongest laser in the smallest space. Note that the figure shows the fundamental transverse mode only which is often the assumption in laser diode models.

\subsubsection{Spontaneous Emission}

Although laser operation is based on stimulated emission, it should be noted that without spontaneous emission the optical signal gain could not get started. The source of energy of a laser is the pump and it will raise the energy of the carriers so that they can drop between the critical energy gap of which the entire laser is designed. Once the first few photons are emitted at the laser designed wavelength, stimulated emission will begin because of the population inversion maintained by the pump. 


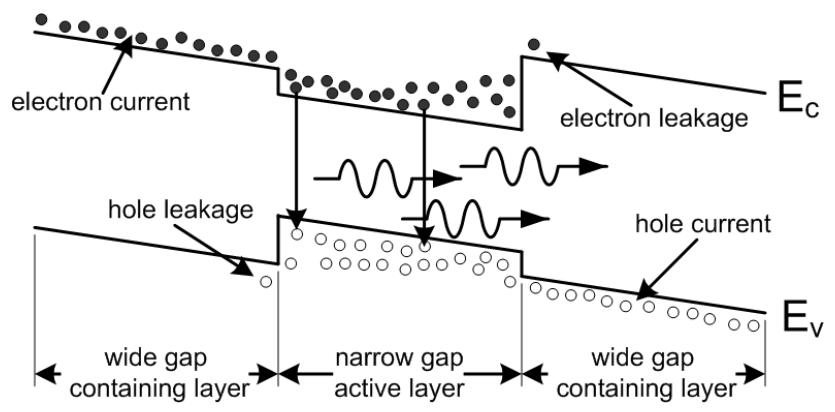

Figure 2.16: Semiconductor laser band diagram under forward bias showing how the layers of material with different indexes of refraction also create a waveguide for photon confinement.

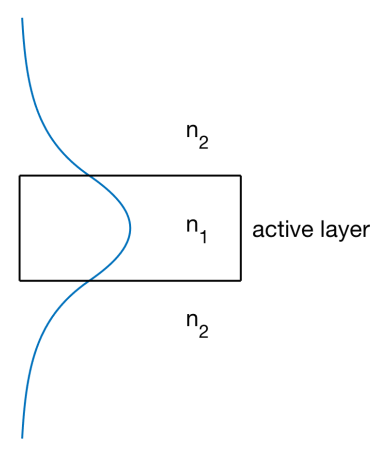

Figure 2.17: Photons are confined to the active layer.

\subsubsection{Resonant Cavity}

As mentioned above we need optical feedback to allow the gain medium to continue to amplify the stimulated emission. The simplest method is with mirrors. They can be implemented by simple facets or usually with gratings but the effect is the same - the optical signal is amplified again and again with each pass through the cavity. As the signal passes through the cavity it will interfere with itself. The requirement is that the end points of the signal meet - or there are $2 \pi m$ radians in the cavity. So even though the ends of the cavity do not force the electric field to zero the number of wavelengths in the cavity must be an integral number or they will interfere and cancel. We therefore have the same condition as a cavity where the ends do force the electric field to zero. As in (2.36) we again have

$$
L=\frac{m \lambda}{2}
$$

So now we have a situation where the radiation will fall within a narrow spectrum as illustrated in figure 2.15 but is restricted to certain wavelengths by the cavity (see figure 2.18). This means we will have a relative intensity where the two spectra overlap. This is 


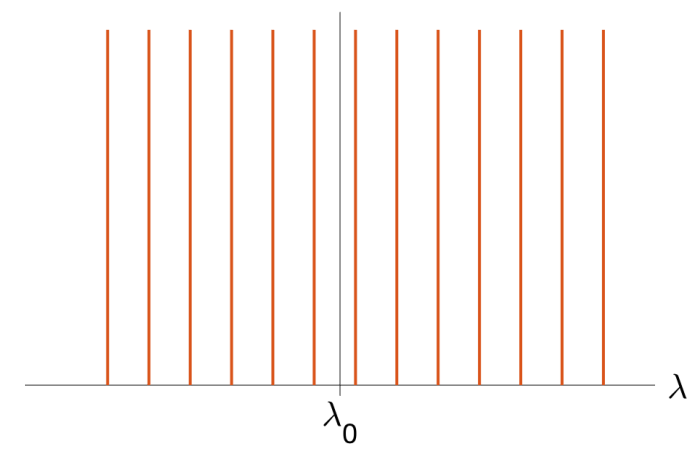

Figure 2.18: Semiconductor laser allowed wavelengths. The spacing between these is $2 L$.

shown in figure 2.19. Note that in this example the peak of the stimulated emission does not line up with an allowed wavelength.

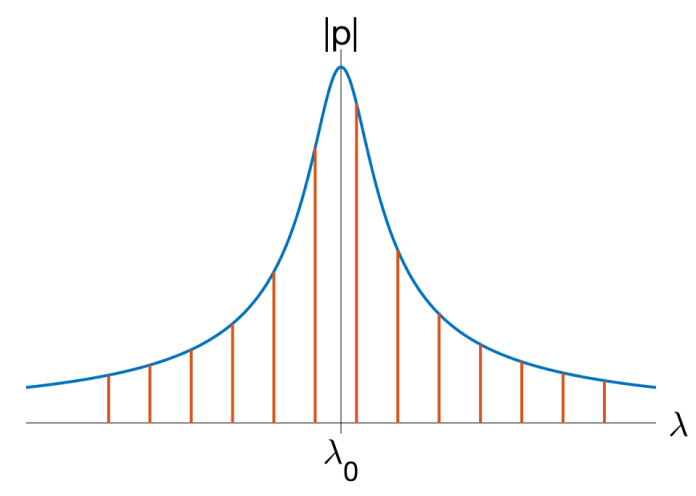

Figure 2.19: Semiconductor laser relative intensity of the allowed wavelengths.

\subsubsection{Laser Modes}

The modes in a laser are determined by the physical geometry and the excitation. Any modes that are excited by the laser sources and permitted by the geometry will be present. This will be a mix of mode types and mode numbers. Most lasers are designed to support the fundamental mode so it will typically be the strongest. This would be the TE10 or TE01 and TM10 or TM01 depending on the geometry. The cutoff frequency has to be considered but again, usually lasers are designed to be single mode. The fundamental transverse mode 
was already shown in figure 2.17 .

The length of the resonant cavity will determine the fundamental longitudinal mode and this is also typically designed to be the lasing frequency. Figure 2.18 shows the cavity longitudinal modes. It is not always possible to match the stimulated emission frequency with the cavity so as illustrated in figure 2.19 we see that the fundamental mode, even though it is not the same frequency as the stimulated emission, is still the mode with the most power. This figure also illustrates that all of the modes that are excited will be present.

\subsubsection{Electrical Properties}

Because the semiconductor laser is constructed like an LED the electrical properties are the same and straightforward - it will appear as a diode. The current-voltage curve will be an exponential. In a schematic it will behave like an LED with two terminals. See figure 2.20. Internal to the laser device is a compact model made up of circuit elements where possible.

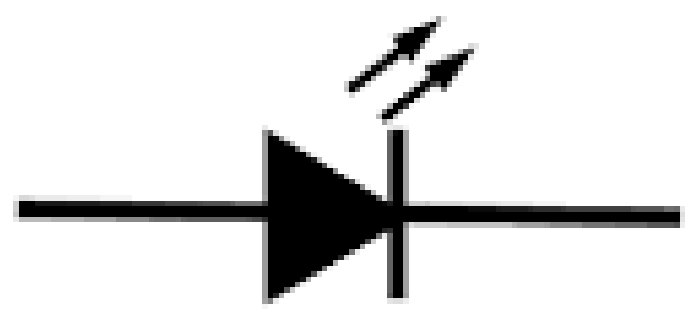

Figure 2.20: Semiconductor laser symbol—same as an LED.

The compact model for a laser must also interface to the optical domain. In figure 2.21 the laser model block contains the equations used to generate the optical field. These equations take the current from the electronic circuit as an input and feed this into the optical part. This example is using the REM but all of the models we discuss may be substituted here. In the figure a thermal circuit is included which will interface to the thermal domain and affect the temperature. These elements have the 'th' subscript. 


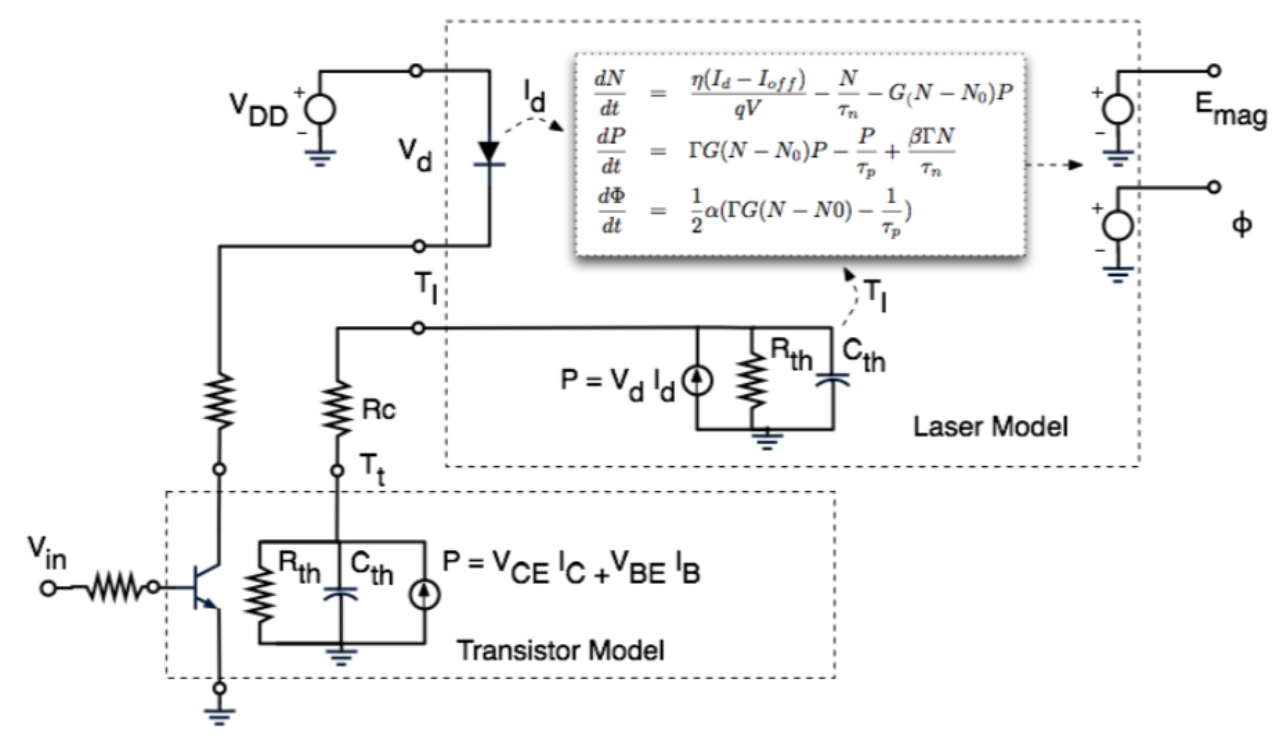

Figure 2.21: Semiconductor laser compact model.

\subsection{Carrier Density}

We understand that the semiconductor laser electrically behaves similar to a diode or LED. For the circuit simulator this is simple to model and is convenient for the MNA formulation. Of course this does not model the optical properties of the laser other than perhaps the total power including optical output and losses. The goal is to combine the optical simulation with the electrical (as outlined in chapter 1). To that end we need to interface the electrical circuit with the optical. Using conservation of energy and conservation of mass we can count the carriers injected and equate this to the photons generated by both stimulated and spontaneous emission as well as the photons lost. This is our interface between the the electrical and optical domains and is captured in the rate equations shown in the laser model box of figure 2.21. A derivation for the REM version of these equations is given in appendix H. The laser model may also be replaced by the TLM or TWM as well.

\subsection{Dispersion}

Dispersion is the frequency dependence of the refractive index $(n)$ and causes different frequencies to travel at different speeds. [8] This is due to the polarization of the material-all of the electrons in the material move in response to the electric field. At the macroscopic level light going through a dielectric medium appears to travel slower in the presence of the material. If we use the Classic Electron Oscillator to model the material the incident field will drive the electrons at the same frequency. The oscillator will have a resonant frequency $\omega_{0}$ that the material responds to more than any other. The light will come in at one phase 
and drive the electrons which then radiate at a different phase which when added to the source will appear as a delay so the field appears retarded. For transparent materials with an index close to 1 this delay will manifest as a phase velocity less than the speed of light.

We can quantify this by working out the field these polarized atoms will produce and extract the index of refraction

$$
n^{2}=1+\frac{N q_{e}^{2}}{2 \epsilon_{0} m\left(\omega_{0}^{2}-\omega^{2}\right)}
$$

where $N$ is the number of molecules per unit volume. This is the theoretical dispersion relation showing how the atomic behavior causes the field to appear retarded. A derivation is given in [9] and appendix J.1 gives an intuitive 'explanation' of the index of refraction.

The effect of dispersion is to distort the optical signal so that pulses spread in the time domain. This is shown in figure 2.22. In (2.39) the index is shown as a real number. When concerned with the speed of a wave this is the determining factor. But the index is actually a complex value and also incorporates gain, with the gain peak at the resonant frequency $\omega_{0}$. For many materials this resonant frequency is well above visible light and so at frequencies of interest the index is for all intents and purposes real and close to unity. This is true for transparent materials.

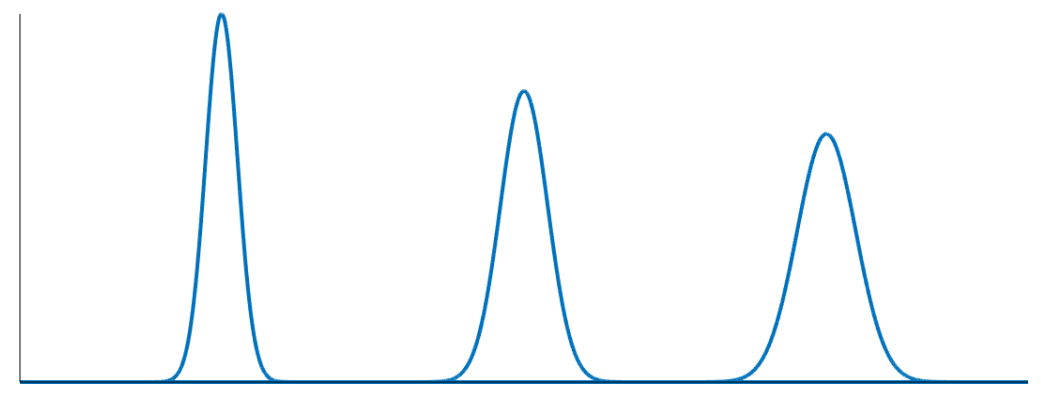

Figure 2.22: Spread of pulse in time domain due to dispersive medium. Same pulse moving in time. Shortens and widens as it moves.

\subsection{Henry Factor}

When a light wave propagates through the active layer with gain the electric field will have phase $(\beta)$ and gain $(g)$ and a plane wave will have the following form

$$
E(z)=E_{0} e^{-i \beta z} e^{g z}
$$

where the carrier has been suppressed. The Kramers-Krönig relations show that $\beta$ and $g$ are linked and so also are the real and imaginary part of the complex refractive index. 
$\alpha_{H}$, the Henry factor (called this after C.H.Henry wrote his paper [10]), is calculated as the change in the real part of $n$ over the imaginary part of $n$ as $N$ is changed. This is assumed to be from $N_{t r}$, the carrier density at transparency, but may also be at another point such as threshold. It is important that the carrier density point and frequency be specified.

$$
\begin{aligned}
\alpha_{H} & \simeq-\frac{\triangle n_{r}}{\triangle n_{i}} \\
& =-\frac{2 \pi \triangle n_{r}}{\lambda_{f s} \triangle g}
\end{aligned}
$$

Rearranging we get

$$
\triangle n_{r}=-\alpha_{H} \frac{\lambda_{f s}}{2 \pi} \triangle g
$$

which shows how this approximation gives the change in the real part of the index of refraction produced by a change in gain. This approximation is linear at the specific carrier density and frequency. The change in the index will change the optical length of the laser and therefore the carrier frequency. This is undesirable in lasers (especially communication lasers) and is called chirp. Given that

$$
\frac{\triangle f}{f} \simeq-\frac{\triangle n}{n}
$$

(see section F.4) and using (2.42)

$$
\frac{\triangle f}{f} \sim-\frac{\overline{\triangle n_{r}}}{n_{r}} \sim \frac{\lambda_{f s}}{2 \pi} \overline{\alpha_{H} \triangle g}
$$

In section F.4 we derive these relationships. If we take a sample index of refraction we can see how $\alpha_{H}$ will vary with frequency. Refer to figure 2.23. In DFB lasers the operating frequency is determined more by the grating. This allows the designer to choose a frequency with a lower $\alpha_{H}$ thereby reducing the chirp. 

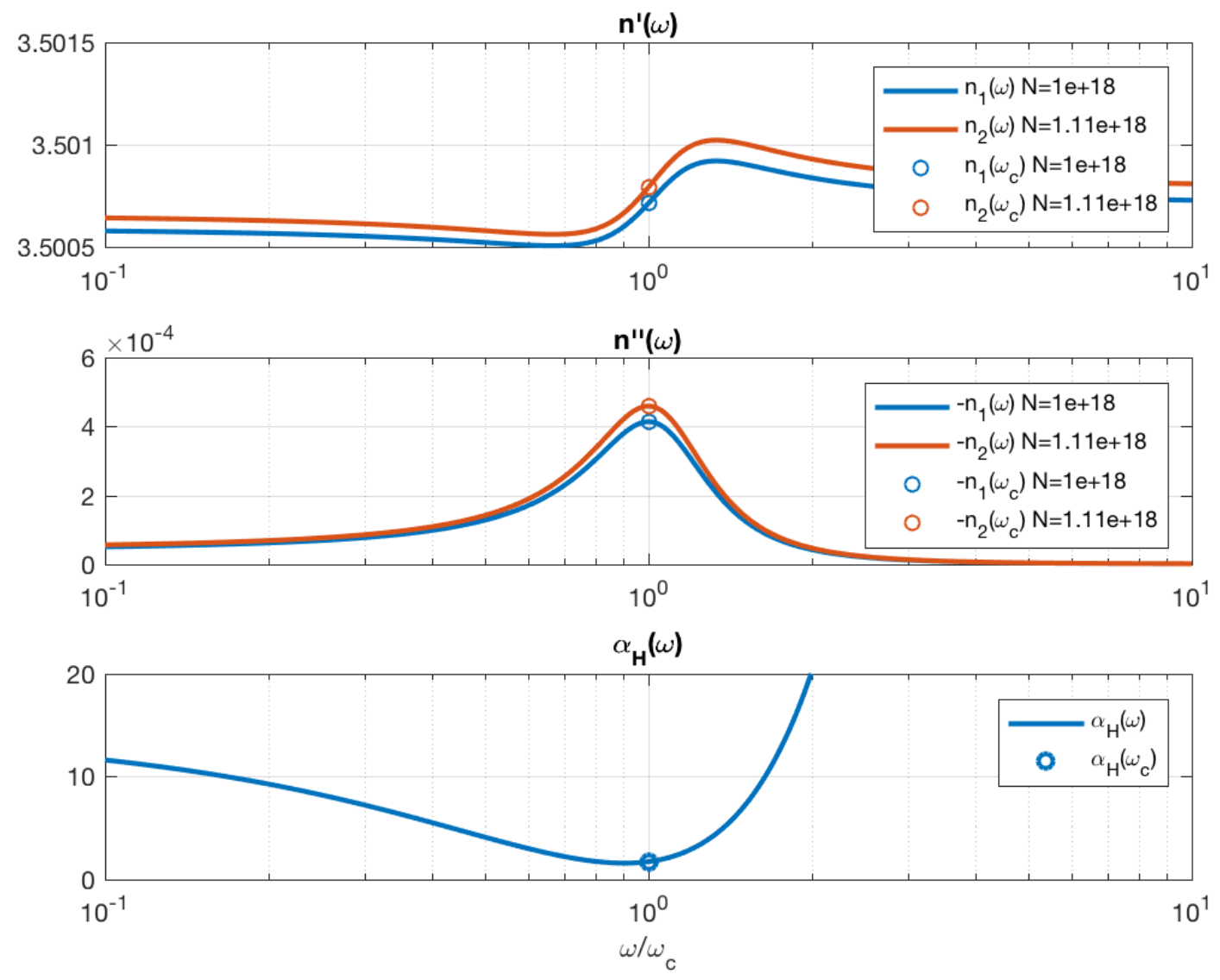

Figure 2.23: Henry factor $\left(\alpha_{H}\right)$ over frequency for sample index of refraction. 


\section{Chapter 3}

\section{Laser Modeling}

This chapter summarizes the most common compact laser models. With the goal in mind to see if it is reasonable and worthwhile to recommend another model over the classic REM for use in our commercial SPICE-like simulator we spend some time going over this model. It is well studied and certainly has merit and is easy to incorporate into an MNA engine for numerical computation. The evolution of this model is also reviewed as the classic REM is no longer adequate for our intended use in a commercial simulator. The type of model we are looking for is a compact model because full-wave electromagnetic analysis, although very accurate, is very computationally intensive and not suitable for design simulations. Compact models do not model the laser directly with Maxwell's equations or at that level of detail but attempt a reasonably accurate model that is fast enough to insert into the simulator; what is reasonable and what is fast enough are not the same for every user. The compact models discussed here all implement a set of DEs that approximate the behaviour of the system variables being modeled but differ in how this is done. The TLM is only briefly presented as it was previously analyzed. The conclusions of that analysis are reviewed. The TWM is looked at in detail. Some background on the laser equations are found in [11], [12]. The laser parameters for our models come from various papers. Two of the main ones are [13], [14]

\subsection{Complex Envelope}

We take a look at the complex number representation for the model because this is a simple way to store the phase and magnitude information for MNA SPICE-like engines. It would be difficult for the MNA engines to handle the high frequency carrier and it is the information carried by the optical signal that is at the same frequencies as the electronic signals - the much higher carrier signals are of no interest. Therefore we would like to use the complex envelope in our model. It is a powerful way to reduce the overhead of dealing with the carrier while still maintaining the characteristics of a signal on a carrier. A good example to illustrate this is interference of optical signals where two signals should only add if in phase. If only real numbers were used the signals would only interfere if the carrier information were included 
as well. This is a lot of overhead especially when the carrier really only contains the relative phase information. By using a complex number envelope the carrier phase is contained in the signal. Whenever a measurable real-world signal is required it is simply generated from the complex number representation. In this way the model then only handles the envelope of the optical signals and the complex number representation automatically keeps track of the carrier phase. The major advantage of this is simulation time. Because the envelope changes much slower than the carrier we can reduce the time step of the simulation. It also greatly reduces the amount of data required to store and manipulate. There are certain complications that arise from using the complex envelope and it is easy to forget the carrier completely. As an example a typical electric field is specified by

$$
E(z, t)=M(z, t) e^{i(k z-\omega t+\phi(z, t))}
$$

where $M(t)$ and $\phi(t)$ are the magnitude and phase shown with the carrier. In the complex envelope representation this is represented by only the magnitude and phase as a complex number

$$
E(z, t)=M(z, t) e^{i \phi(z, t)}
$$

where the carrier has been removed and is now implicitly understood. It still has the correct properties such that when two electromagnetic waves are added the interference is handled correctly by the complex number mathematics. To get the electromagnetic wave intensity we can treat the complex envelope as the actual wave because when it is multiplied by its complex conjugate we get the same result as if we had added the carrier term back and used the full representation in the calculation. This happens of course because the carrier term will cancel with its conjugate. So far this seems obvious and might even seem trivial. Indeed, this type of simplification is typically done; we take two modulated signals and add them without concern and expect the correct result. There are two cases where this is not the case - when the carrier frequencies are not the same or even if they are, when they are not in phase.

\subsubsection{Timing Reference}

Before discussing the details of the complex envelope examples we need to clarify what we will use as a signal reference. Regardless of the carrier and modulation chosen there is also a signal which all others are compared to - this is the reference. This is a point in time from which all other signals are measured relative to. Note that it is valid for all space. System simulations for which different points in space are compared to each other must use the same reference for the timing information to be valid. For system simulations where signals are being combined not only must they use the same timing reference, but of course you must ensure that they are measured at the same location as well. This last part is usually not 
a problem because the physical circuit dictates the locations of all signals. Because of the distributed nature of the TWM, however, its traveling waves, which of course move in space, can be confusing when comparing to a reference wave. Hopefully this will become clearer with a few examples.

\subsubsection{Calculating Envelope Phase}

Phase can be calculated given any arbitrary signal or by calculating the relative phase between two signals. For an overview of phase calculations refer to A.1.

\subsubsection{Envelope Phase and Time Delay}

There is a consequence of not including the carrier in the optical signal model calculations. With normal electical signals they are propagated and will always have a common reference point as all inputs are referenced to the same point in time and each device will modify the signal always including at least some delay. The outputs can be compared as they are calculated. If the full optical signal, including carrier, were propagated this would be the case for our optical simulation as well. But because we only do calculations with the envelope this is not the case for our models. As specified above in section 3.1.1 we still have a common timing reference, but since only the envelope is propagated in the model the phase information is no longer correct. This is because the carrier has also been delayed and was not taken into account. Examples of this are given in appendixes A.2, A.3 and A.4 and show that for system simulations or simulations involving two TWMs a separate detuning factor must be sent along with the carrier envelope to keep track of the relative phase. This is one of those times it is easy to forget about the carrier altogether resulting in incorrect results.

\subsubsection{Carriers not in Phase}

When the carriers of two signals are not in phase they cannot be added directly. In this case the carrier phase information must be accounted for in the complex envelope. If the phase is constant this is easily done by including a phase factor. Here are two complex envelopes shown with the carrier

$$
\begin{array}{r}
E_{1}=M_{1}(z, t) e^{-i \omega_{c} t} \\
E_{2}=M_{2}(z, t) e^{i\left(-\omega_{c} t+\delta\right)}
\end{array}
$$

where the first carrier phase was chosen as the reference. Now show $E_{2}$ with the carrier phase moved into the envelope

$$
E_{2}=e^{i \delta} M_{2}(z, t) e^{-i \omega_{c} t}
$$


Adding these two signals we get

$$
E=E_{1}+E_{2}=\left(M_{1}(z, t)+e^{i \delta} M_{2}(z, t)\right) e^{-i \omega_{c} t}
$$

With the carrier phase now expressed in the complex envelope we can dispense with the carriers and safely suppress them in the notation and add the envelopes directly.

$$
E=M_{1}(z, t)+e^{i \delta} M_{2}(z, t)
$$

This is how we would like to work with the complex envelope; given two constant amplitude envelopes with carriers 180 degrees out of phase we would have two complex numbers $E_{1}=1+0 i$ and $E_{2}=e^{i \pi}=-1+0 i$ so that when we add them the interference is handled by the complex number representation and they cancel as $E_{1}+E_{2}=1+0 i+(-1+0 i)=0$.

As an example of how this is not a trivial situation consider the delay of a signal through a waveguide. It is not enough to use the model and generate a new output signal with amplitude and phase. This is because the carrier has been delayed as well as the envelope. If we had two different length waveguides and propagated the signal through both and added, the model would give us double the amplitude and ignore the carrier phase change - the modulation phase was handled correctly, the carrier phase was not. This example is detailed in appendix A.3.

\subsubsection{Different Carrier Frequencies}

When the carriers are not the same frequency it should be clear that we cannot add their complex envelopes and expect a meaningful result. This can occur during system simulations when the outputs of two models are brought together. In this case the carrier phase information must be accounted for in the complex envelope. If we choose the first signal as the reference with carrier at frequency $\omega_{c}$ we have

$$
E_{\text {ref }}=e^{-i \omega_{c} t}
$$

The second signal at frequency $\omega_{1}$ is

$$
E_{1}=M(z, t) e^{-i \omega_{1} t}
$$

We wish to have $E 1$ with respect to our reference so

$$
E_{1}=\frac{M(z, t) e^{-i \omega_{1} t}}{e^{-i \omega_{c} t}} e^{-i \omega_{c} t}=M(z, t) e^{-i \delta t} e^{-i \omega_{c} t}=M^{\prime}(z, t) e^{-i \omega_{c} t}
$$

where $\delta=\omega_{1}-\omega_{c}$. Note that the phase factor is time-varying. This example is detailed in appendix A.5. 


\subsection{Rate Equation Model}

The first model we will look at is the Rate Equation Model (REM). The REM is based on a pair of cross-coupled differential equations that govern how the carrier concentration $(N)$ and the photon density $(S)$ change - equations (3.9-3.10) below. This basic model is often referred to as the classic rate equations. It is then typically augmented with a third phase equation: (3.11). This equation represents the phase of the envelope. [15] [13]

$$
\begin{aligned}
& \frac{d N}{d t}=\frac{I}{q V}-g_{0}\left(N-N_{t r}\right) \frac{S}{1+\epsilon S}-\frac{N}{\tau_{n}} \\
& \frac{d S}{d t}=\Gamma g_{0}\left(N-N_{t r}\right) \frac{S}{1+\epsilon S}-\frac{S}{\tau_{p}}+\frac{\Gamma \beta N}{\tau_{n}} \\
& \frac{d \phi}{d t}=\frac{\alpha_{H}}{2}\left[\Gamma g_{0}\left(N-N_{t r}\right)-\frac{1}{\tau_{p}}\right]
\end{aligned}
$$

The REM is a lumped model in that there is only one average value of $N$ and $S$ for the entire laser. It has been used extensively because it is very simple and gives reasonable laser dynamics with little computational cost. The index of refraction is averaged over the entire active layer and an effective index is used for the laser.

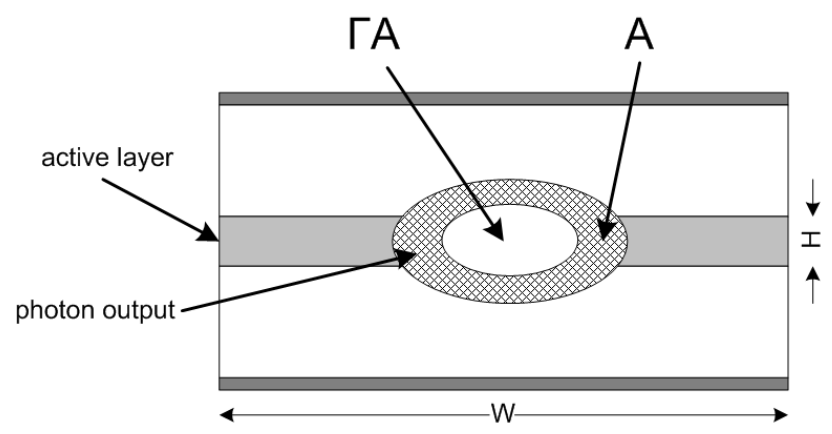

Figure 3.1: Active layer showing fields, confinement factor $(\Gamma) . A$ is area of field. $\Gamma A$ is area of field interacting with carriers.

The first two of these cross-coupled DEs are derived in appendix $\mathrm{H}$ where the confinement factor and confinement area (shown in figure 3.1) are explained. It is important to note that $N$ is not the absolute carrier density, but rather the number of excess carriers. For a highly doped semiconductor this would be the level above the doping level $N_{d}$. This model does not deal with holes explicitly, the assumption being that they essentially mirror the electrons.

From (3.9) we can see that with no current $(I)$ and no photons $(S), N$ will decay exponentially to 0 with a time constant $\tau_{p}$ called the carrier lifetime. Recombination is responsible for this process. This is the same state we would find a doped semiconductor not connected in a circuit or exposed to light. $N_{t r}$ is the carrier transparency level. At this level the optical gain just balances the absorption in the active medium so the net gain is unity within the active medium. Since there is no optical gain or loss, the medium behaves as if it were 
transparent. [16] The second term in (3.9) is the stimulated emission term. It is proportional to the number of photons but of course stimulated emission absorbs carriers so there is a negative sign. Since $N$ is the number of electrons in the conduction band, it is the upper level concentration in a two-level system. $N>N_{t r}$ indicates a population inversion; we have stimulated emission and a gain in the medium so the number of photons grows. We can contrast $N_{t r}$, transparency with $N_{t h}$, threshold. Threshold is defined as when the laser starts to lase. It is always greater than transparency because we need $N>N_{t r}$ for stimulated emission which we need to lase. When the stimulated emission overcomes the losses of the system it will start to lase; this is threshold. Note that threshold is a carrier level $\left(N_{t h}\right)$ where $\frac{d S}{d t}=0$.

$g_{0}$, the gain slope constant scales the stimulated emission. $\epsilon$, the gain compression factor is discussed later in section 3.4.

$q$ is the charge per electron (carrier) and $V$ is the volume of the active layer. Knowing the current will then give us the injected carriers per volume per second. If there were no losses $N$ would grow at this rate.

$S$, the photon density is equivalent to the light intensity. This is directly related to the electromagnetic field.

In (3.10) we see a factor $\tau_{p}$, the photon lifetime, which accounts for the exponential decay of the number of photons in the absence of other processes. We also see the same stimulated emission term only this time it is a positive term. This is because a gain in the medium will cause the photon density to increase. The last term in (3.10) is the spontaneous emission term. This is the number of photons that are emitted through random incoherent radiation. It is like the recombination term in the $N$ equation but with a factor $\beta$, the fraction of the spontaneous emission coupled into the lasing mode. It is small and we will ignore most of the spontaneous emission as it will not contribute to lasing. $\Gamma$ is the confinement factor; the fraction of the radiation that is within the active layer. It is this portion that will interact with the population of carriers. The transverse modes of the laser are never confined completely in the active layer so this factor also takes this into account. For an explanation on the physics behind the confinement factor see section 2.3.4. Note that this affects the stimulated and spontaneous emission terms.

The last of the differential equations (3.11) specifies the phase of the radiation. Note that it is not part of the two cross-coupled DEs; it is actually an output. It is not always used with rate equation models but was added later. It refers to the complex phase of the envelope and is based on the stimulated emission and recombination terms of the $S$ equation. It shows that there will be a deviation from the carrier frequency centered around threshold. It is directly dependent on $\alpha_{H}$ the Henry factor. (See section 2.6).

Examining these equations you will notice that they do not explicity use the carrier frequency. As mentioned above this is a great savings computationally. The carrier frequency is known but not used for the laser dynamics. All modulation is referenced to this frequency. Historically this was not a problem because the model was used standalone to understand laser dynamics. This has consequences for system simulations when two models are run side by side as mentioned earlier. 
A final thing to note about the REM is that it is single-mode. This assumes only one longitudinal mode or wavelength exists in the laser which means there is no frequency dependence and so there is no dispersion. Overall it makes the model simple but of course is not always true so limits its application.

\subsection{Rate Equation Model Basic Dynamics}

The basic dynamics of the REM are captured in the first two cross-coupled equations (3.9), and (3.10) reproduced here for convenience (omitting the spontaneous emission term). We review the dynamics of the model during startup.

$$
\begin{aligned}
& \frac{d N}{d t}=\frac{I}{e V}-g_{0}\left(N-N_{t r}\right) S-\frac{N}{\tau_{n}} \\
& \frac{d S}{d t}=\Gamma g_{0}\left(N-N_{t r}\right) S-\frac{S}{\tau_{p}}
\end{aligned}
$$
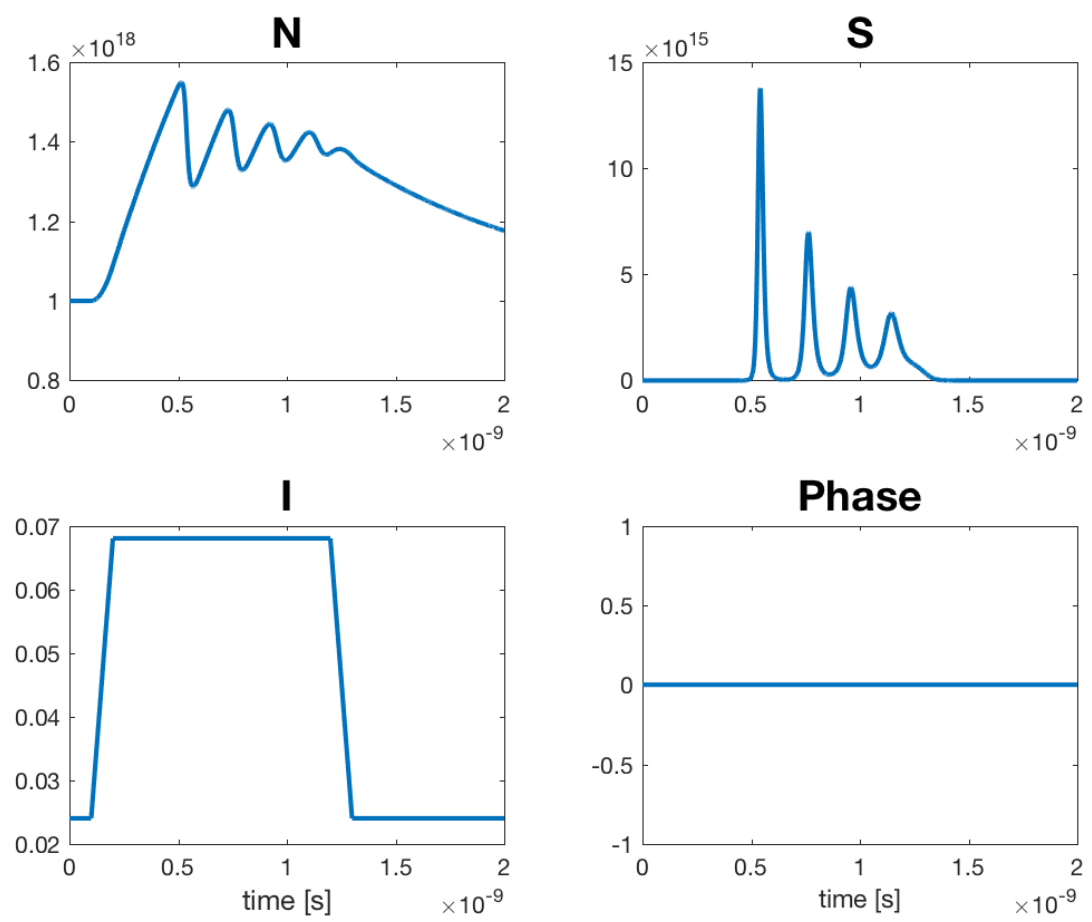

Figure 3.2: REM current pulse simulation, $N$ starting at $N_{t r} . N$ and $S$ have units $\mathrm{cm}^{-3}$.

We start with no photons, $N$ at transparency $\left(N_{t r}\right)$ and enough current to keep $N$ at transparency. If we had no current the first two terms of (3.12) would be zero and we 
would get the exponential decay according to the recombination lifetime $\tau_{n}$. We stay at transparency until the current source is turned on and 'pumps' the laser. The number of photons will stay at 0 because both terms of (3.13) are 0 . Refer to figure 3.2. Once the current source is switched on the carrier density $N$ starts to build linearly. This continues until we reach threshold. Notice that this is greater than transparency because the photon density is low and the stimulated emission term negligible. After $S$ overcomes the losses and reaches threshold the stimulated emission has an avalanche effect producing optical gain but sweeping out the carriers; $S$ shoots up while $N$ drops. Once $N$ drops below transparency the photons, having a short lifetime, are quickly lost and we are back to a similar situation that we started with. The current then starts to build $N$ and the cycle starts over. We can see from the figure that this will continue until a steady state is reached. In this example, however, the current source is turned off and both $N$ and $P$ decay. $P$ is much faster and reaches 0 . This is a typical example of direct modulation of a laser; the light intensity out will be linearly related to the current pulse in.
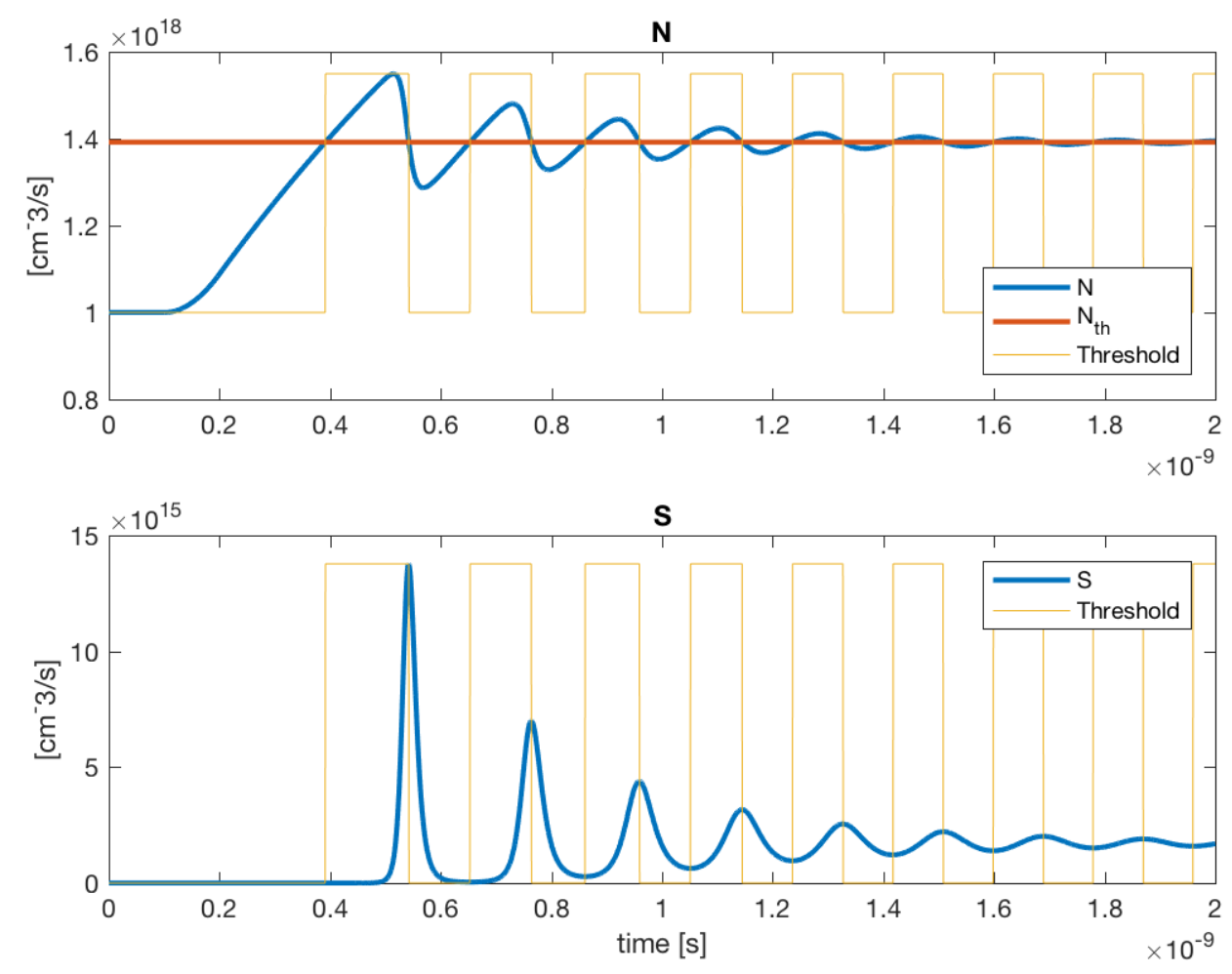

Figure 3.3: Transient plots of REM DE terms during system startup which then settles at threshold. Carrier and photon densities $N$ and $S$ units are $\mathrm{cm}^{-3}$

Let's look at a more detailed example plotting some of the DE terms. See figures 3.3-3.8. Again starting at transparency with $N=N_{t r}$ and $S=0, N$ will build approximately linearly with $I$ and $S=0$ (figure 3.3 top). Note that in all these figures we have a signal 'Threshold' 


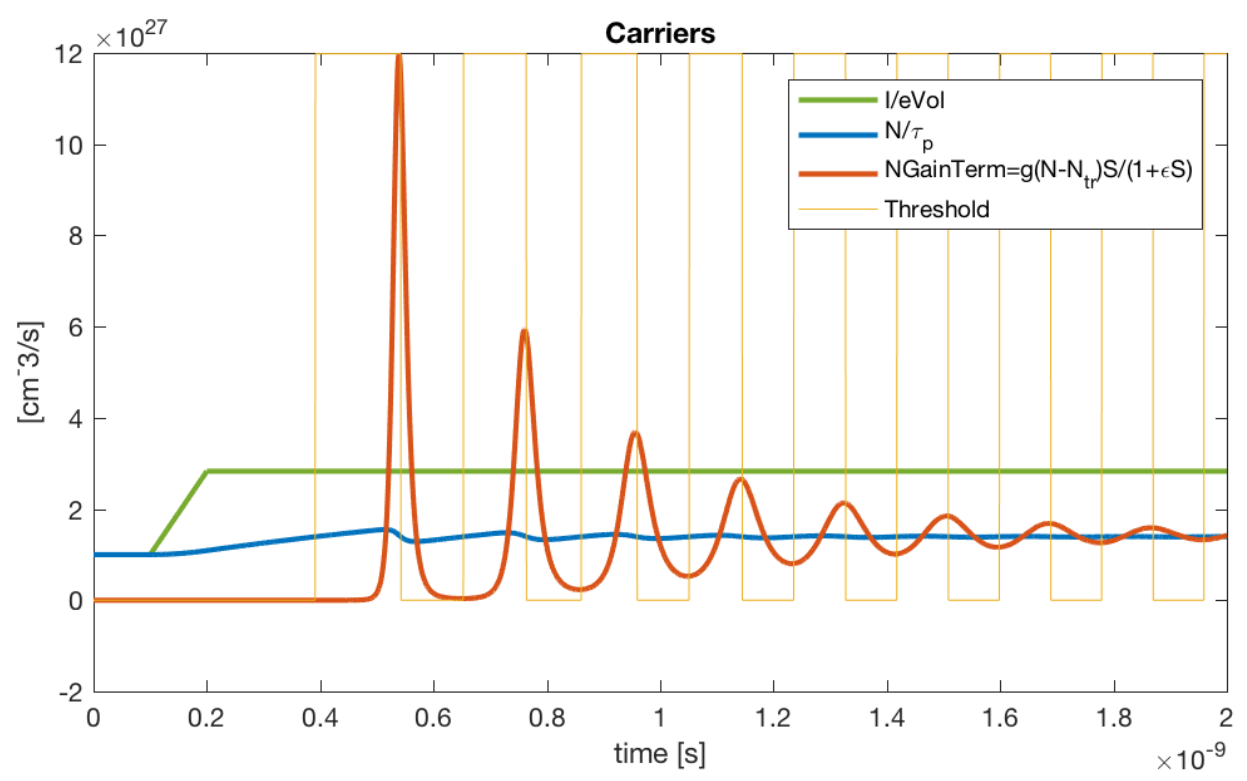

Figure 3.4: Plot of REM DE $N$ equation terms during system startup which then settles at threshold. Carrier density $N$ units are $\mathrm{cm}^{-3}$

which just indicates if $N>N_{t h}$. This allows us to see when we are above threshold and to compare across the different plots. At transparency $\left(N=N_{t r}\right), \frac{d S}{d t}$ is still 0 because of the $\tau_{p}$ term (figure 3.5). At threshold $\frac{d S}{d t}$ becomes positive and $S$ increases faster and faster (figure 3.7). At some point $S$ will be large enough to really affect $\frac{d N}{d t}$ which will then go negative, eventually going below threshold and quickly reducing $S$. In figure 3.3) we see the peak of $S$ and then $N$ is reduced taking us below threshold starting the cycle over again. If the system is damped, it will settle at threshold. Note that $S$ does not increase dramatically until $N$ is above threshold, and $S$ increases until $N$ goes below threshold. Again, use the 'Threshold' signal in the plots to align the signals.

In figure 3.4 we see the $N$ gain term controlling $\frac{d N}{d t}$. When it shoots up with $S$ this quickly reduces $N$. In 3.5 we that the $S$ gain term is almost balanced by the recombination term - it only takes a small difference for the photon level to quickly increase. This is due to the faster response time of the photons. In figure 3.6 we see the same as figure 3.4 with the $I$ and $N$ terms combined showing that the $N$ gain term overwhelms them due to stimulated emission $(S)$. Finally figures 3.7 and 3.8 show the derivative terms for both the $N$ and $S$ equations. 


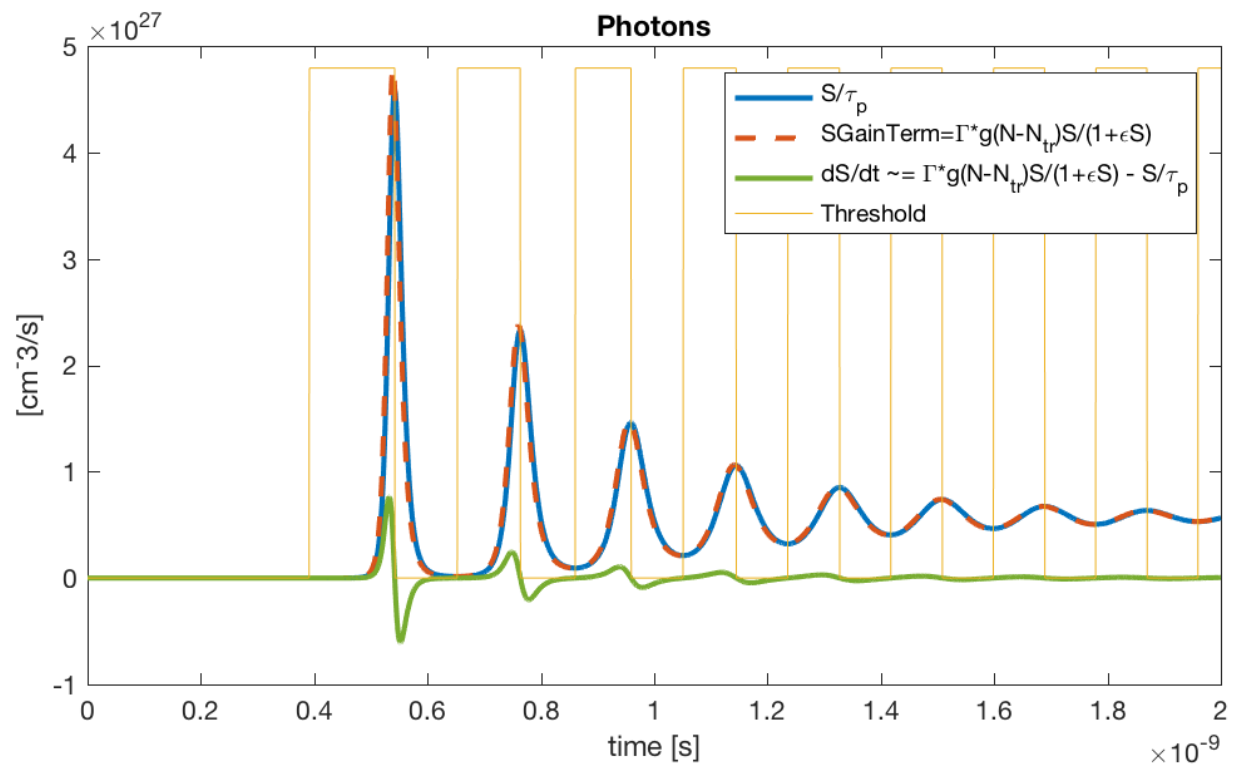

Figure 3.5: Transient plots of REM DE terms during system startup which then settles at threshold. $S$ terms. Photon density $S$ units are $\mathrm{cm}^{-3}$.

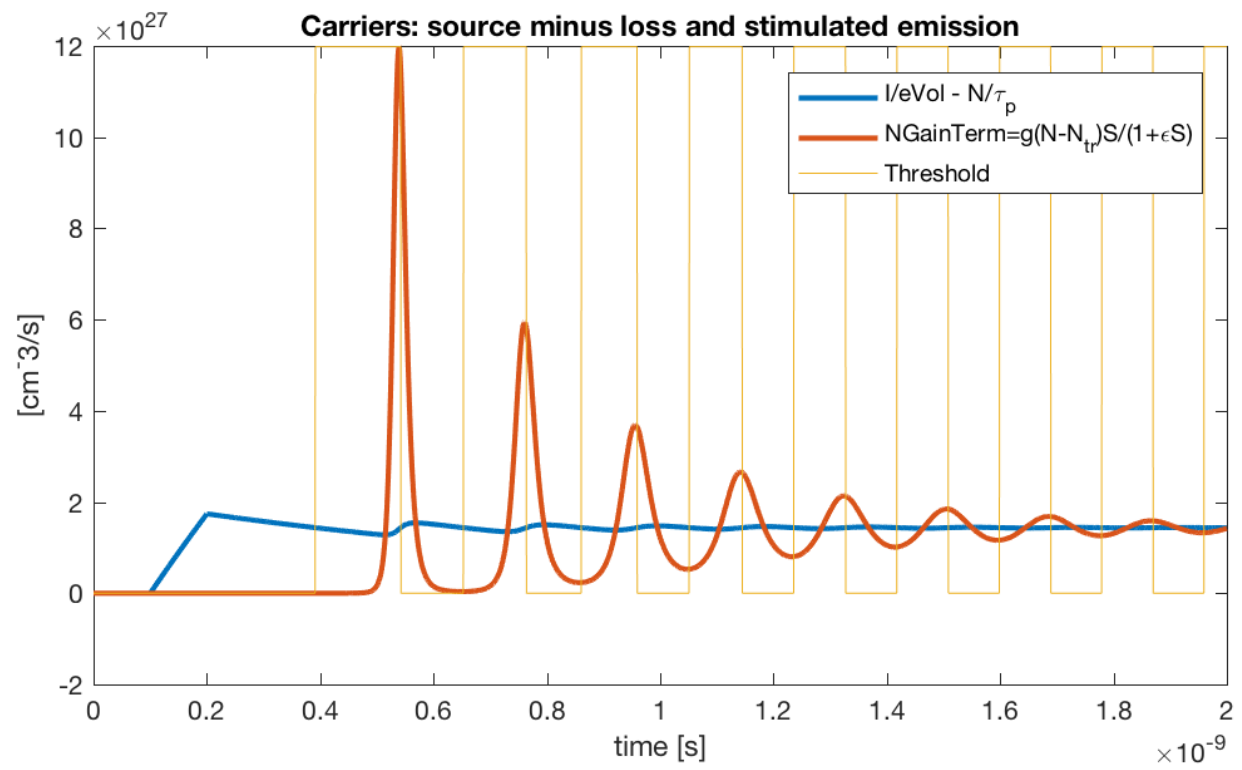

Figure 3.6: Transient plots of REM DE terms during system startup which then settles at threshold. $N$ equation terms with $I$ and $N$ summed. Carrier density $N$ units are $\mathrm{cm}^{-3}$. 


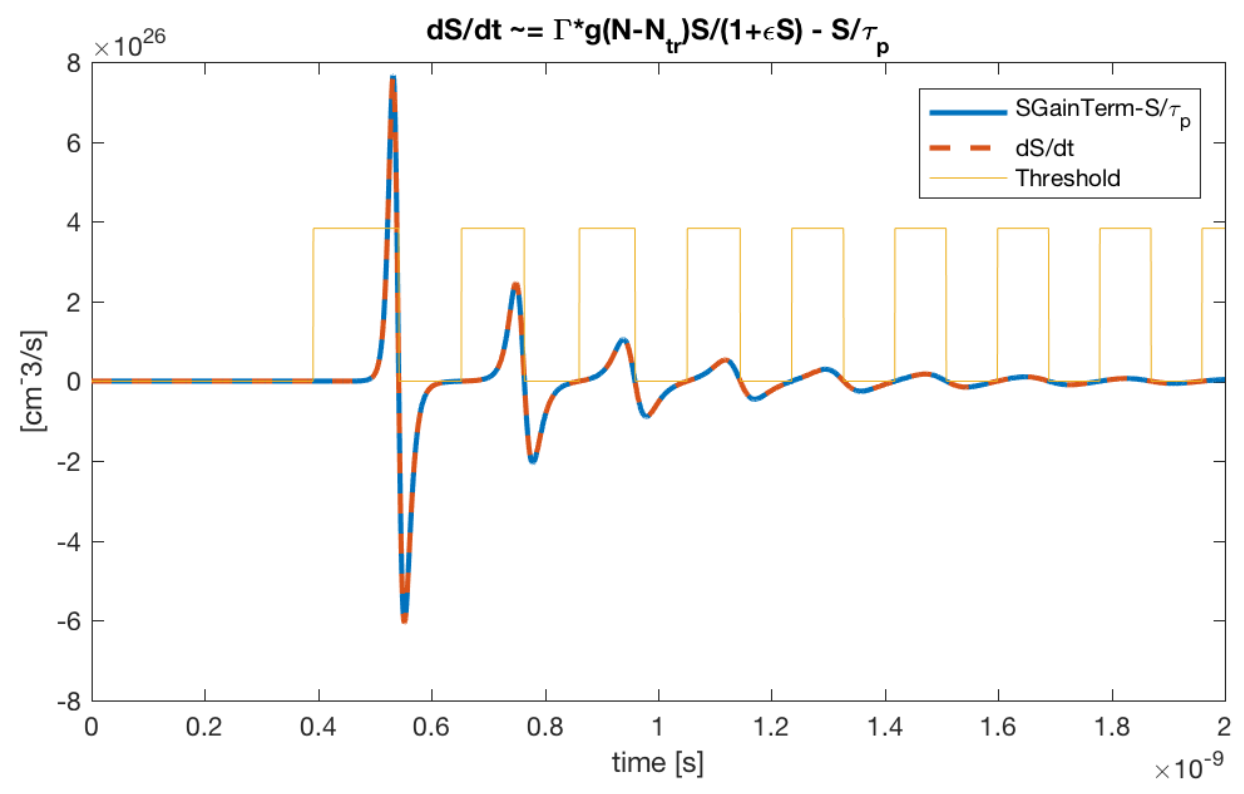

Figure 3.7: Transient plots of REM DE terms during system startup which then settles at threshold. $S$ derivative terms. Photon density $S$ units are $\mathrm{cm}^{-3}$

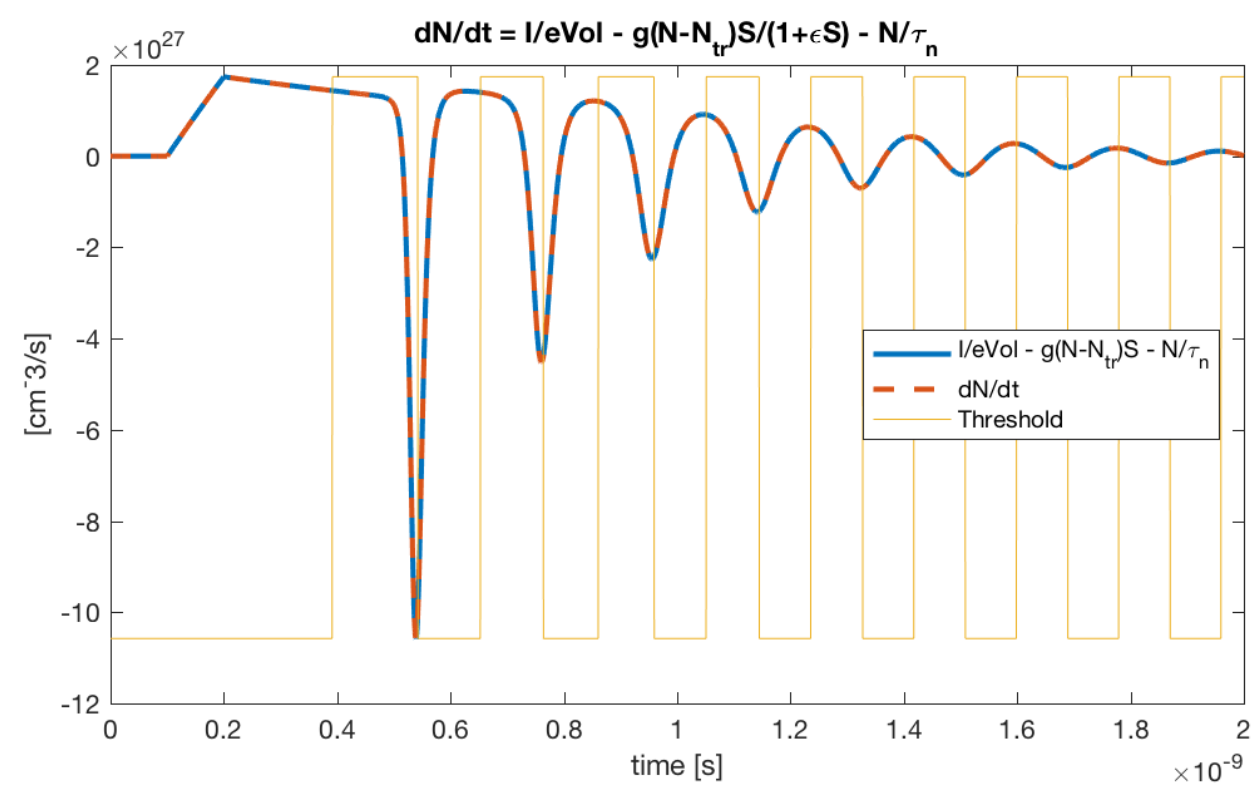

Figure 3.8: Transient plots of REM DE terms during system startup which then settles at threshold. $N$ derivative terms. Carrier density $N$ units are $\mathrm{cm}^{-3}$. 


\subsection{Rate Equation Model With Gain Compression}

Referring to [17] it was recognized that the gain saturates at high photon densities $(S)$ with the dependence $g=\frac{g_{0}}{1+\epsilon}$. The rate equations then become

$$
\begin{aligned}
& \frac{d N}{d t}=\frac{I}{q V}-g_{0}\left(N-N_{t r}\right) \frac{S}{1+\epsilon S}-\frac{N}{\tau_{n}} \\
& \frac{d S}{d t}=\Gamma g_{0}\left(N-N_{t r}\right) \frac{S}{1+\epsilon S}-\frac{S}{\tau_{p}}+\frac{\Gamma \beta N}{\tau_{n}}
\end{aligned}
$$

The change in the REM dynamics is to dampen the photon growth. The effect is quite dramatic and is shown in figure 3.9. This is the same input REM as in figure 3.2 but with the gain compression factor added. It shows that real systems cannot respond with the dynamic range as the original model predicted. Other discrepancies arise [18] but will not be addressed here.
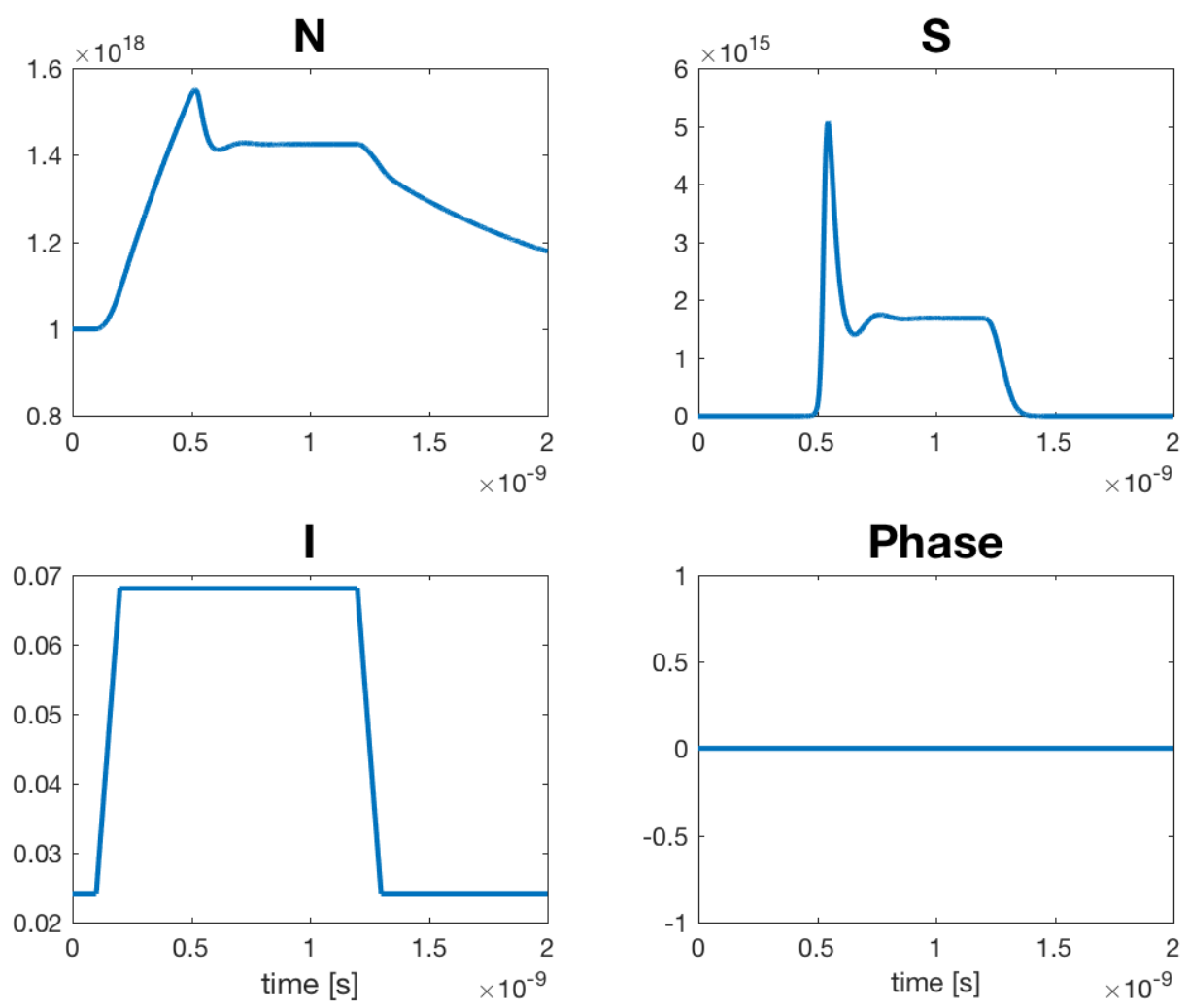

Figure 3.9: REM current pulse simulation, $N$ starting at $N_{t r}, \epsilon=5 \times 10^{-17} . N$ and $S$ have units $\mathrm{cm}^{-3}$ while current is in Amps and phase in radians. 


\subsection{Rate Equation Model With Phase}

The classic rate equations give the average carrier and photon densities for the entire active medium. These were later augmented by the $\phi$ equation (3.16). This equation is not coupled with the others but is really just an output calculated from the $N$ value. See appendix E for a derivation.

$$
\frac{d \phi}{d t}=\frac{\alpha_{H}}{2}\left[\Gamma g_{0}\left(N-N_{t r}\right)-\frac{1}{\tau_{p}}\right]
$$

Remember that $\phi$ is the phase of the modulation on the implicit carrier frequency. If we look at the left hand side $\frac{d \phi}{d t}$ we can see that this is a frequency. If the modulation phase is fixed then $\frac{d \phi}{d t}=\Delta \omega$ will be 0 . If the modulation phase is $\omega_{1} t$, then $\frac{d \phi}{d t}=\Delta \omega=\omega_{1}$. (i.e. $\triangle \omega$ is a frequency and it measures the amount of shift the modulation (envelope) has from the carrier frequency. If we look at (3.16) we can see that $\triangle \omega$ is directly proportional to $N$ and $\alpha_{H}$, the alpha factor or often called the Henry factor (after C. H. Henry who described it in his paper [10]). Refer to section 2.6 for an overciew. If the Henry factor is 0 the shift in frequency of the modulation will also be 0 (implying that at this carrier level the phase is not affected by $N$ ). Otherwise it will track the carrier density $N$. For example, in direct modulation as the current is pulsed $N$ changes significantly causing $\Delta \omega$ to as well. This is a commonly observed phenomena in lasers known as chirp. The Henry factor is a fitted parameter and must be measured around the correct region of $N$, typically threshold. Refer to figure 3.10 for a plot showing phase and chirp. The plots are identical to figure 3.2 except we now have a non-zero phase with an accompanying chirp because a Henry factor other than 0 has been set.

\subsubsection{Chirp and Henry Factor $\alpha_{H}$}

The models that we are dealing with operate at a fixed carrier frequency. This is of course only true in the ideal case. Experimentally, frequency varies as the laser is pulsed. [19] A change in gain with frequency must be associated with change in refractive index. [20] The Kramers-Krönig relations describe how the changes are linked. If we make the assumption that index varies linearly with carrier density then we can calculate a simple factor that defines this linear variation. This is the Henry factor or alpha factor: $\alpha_{H}$ and is discussed in section 2.6.

As the carrier density $N$ moves away from $N_{t r}$ this will create a change in the gain $\triangle g$. Through our factor $\alpha_{H}$, this is linearly converted to a change in frequency-the chirp. This term is defined in the phase equation (3.16):

$$
\alpha_{H} g_{0}\left(N-N_{t r}\right)
$$

The photon lifetime (and confinement factor) will shift the point of zero chirp from transparency to threshold. At $N_{t r}$ the chirp will be negative due to recombination but at $N_{t h}$ it will go positive. i.e. There will be no chirp at threshold. Since the alpha factor is a 

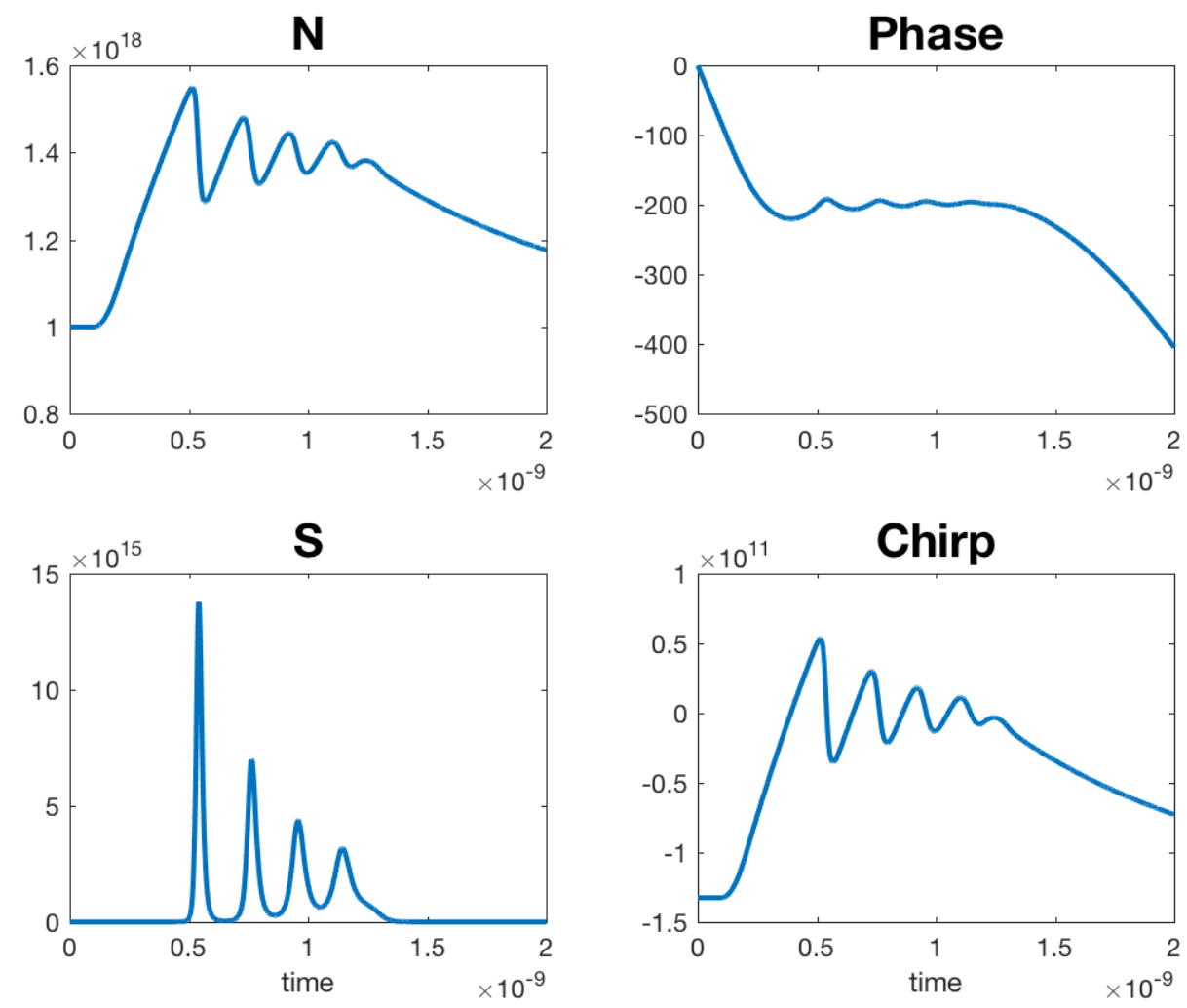

Figure 3.10: REM current pulse simulation, $N$ starting at $N_{t r}$ and showing phase and chirp. $N$ and $S$ have units $\mathrm{cm}^{-3}$ while current is in Amps and phase in radians.

measured parameter it can be measured to whichever $N$ it will be operated at. There is no need to worry about what happens below $N_{t r}$ because no lasing will occur at this level. A derivation of this is given in appendix F.1 followed by an example in F.2.

The Henry factor $\alpha_{H}$ is also known as the linewidth enhancement factor. This is because it was originally derived as a means to explain linewidth broadening which was significantly more than expected theoretically. [10]. It is called linewidth broadening because the dynamic change of frequency (chirp) causes linewidth broadening [20].

\subsection{Multi-mode Rate Equation Model}

The simple single-mode laser model has its obvious limitations - not all lasers operate in single-mode. A more general model is one that can handle all of the modes of a given laser. For background on multi-mode lasers see [21]. For this purpose a basic modification is made to the classic REM. The model first calculates the significant modes of a cavity and treats them all independently. 


$$
\begin{aligned}
\frac{d N}{d t} & =\frac{I}{q V}-\sum_{j} g_{0 j}\left(N-N_{t r}\right) \frac{S_{j}}{1+\epsilon S_{j}}-\frac{N}{\tau_{n}} \\
\frac{d S_{j}}{d t} & =\Gamma g_{0 j}\left(N-N_{t r}\right) \frac{S_{j}}{1+\epsilon S_{j}}-\frac{S}{\tau_{p}}+\frac{\Gamma \beta N}{\tau_{n}}
\end{aligned}
$$

They are then added and the total is plugged into the classic rate equations as shown in (3.18) and (3.19). Note that the frequency response of the modes is also calculated and used to set the $g_{0 j}$ gain parameters. Each mode can have its own index of refraction and therefore group velocity. Example modes are shown in figure 3.11. Left plot shows the emission spectrum while the middle shows the allowed modes. The right plot shows the frequencies and gains that should be used in the model. Any number of modes is permitted but using too many will result in significant computational costs. This is one of the drawbacks that leads one to look for alternative models. This is, however, still the most accurate REM and must be used for multi-mode lasers. [20].
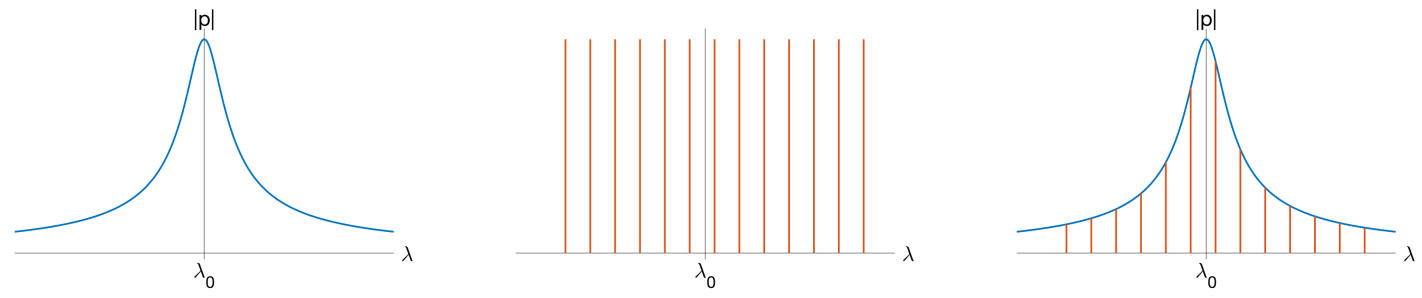

Figure 3.11: Multi-mode spectrum. Left: spectrum of emission. Middle: Allowed laser modes. Right: Resulting multi-mode laser modes with relative intensities. Note: mode $\lambda$ 's are not linearly spaced (approx linear for large $m$ ), but mode freq's are linear. See formula in background section.

Compare the single-mode laser plots in figure 3.2 to the multi-mode laser plots in figures 3.12 and 3.13. We see that the $N$ plots are virtually the same. The dynamics of each mode are similar as expected with the only difference being the power is now distributed across the modes. The phase plots show that the modes are symmetrical. The fundamental mode will be closest to the carrier frequency $\omega_{c}$, while the other modes will come in pairs above and below the fundamental. They will not be perfectly symmetrical with respect to the carrier frequency (i.e. $\pm \omega_{1}$ ) because these modes when combined with the carrier must all be integral number of wavelengths in the laser cavity. We see that the simple lumped model is starting to include spatial considerations by taking into account the laser length for calculation of the allowed modes. The chirp is 0 in these simulations only because the alpha factor is set to zero.

As we increase the number of modes in the multi-mode REM we expect the model to perform better. But compare the $E$-field from runs using 3 and 31 modes in figures 3.14 and 

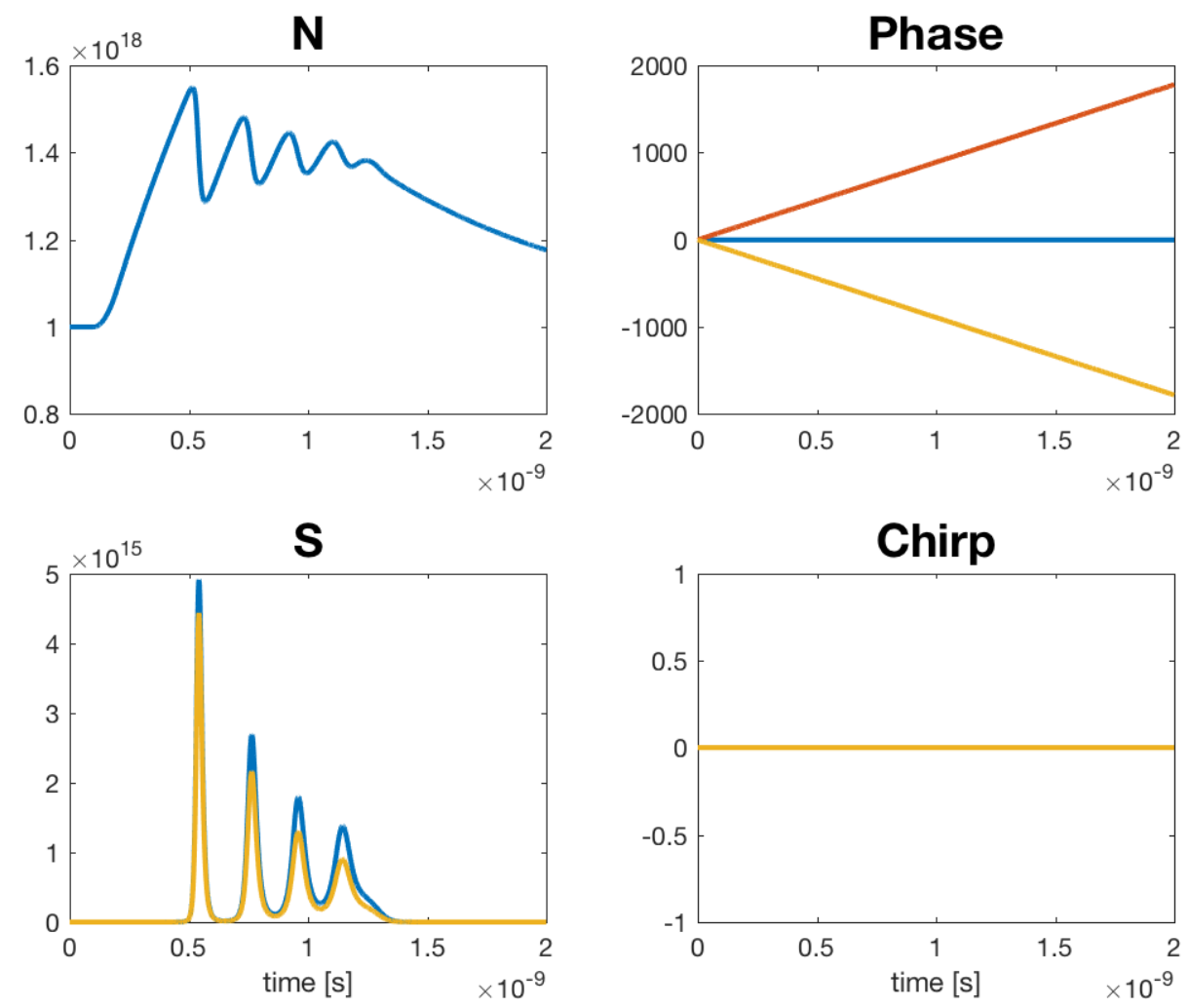

Figure 3.12: Multi-mode REM current pulse simulation, $N$ starting at $N_{t r}$ for 3-mode simulation. $N$ and $S$ have units $\mathrm{cm}^{-3}$ while phase is in radians and chirp rad/s.

3.15. As we add modes the E-field continues to grow. To see what is going on we zoom in. See figure 3.16. As the number of modes increases the pulses narrow and get taller. This is similar to what happens during laser mode-locking. Because the modes are initialized with the same phase we are adding sinusoids of integral multiple frequencies all in phase with each other. The amplitude will build as we increase the number of sinusoids. This is an artifact of the multi-mode REM model and not what happens in a typical laser. This particular defect can be handled by starting each mode with a random phase between $(0,2 \pi)$. With this modification the modes will not be in synchronization and will partially cancel. This is shown in figure 3.17 .

The phase and chirp of the multi-mode model will be examined in chapter 7 . As we will see in section 7.2 the phase of the electric field which is the sum of these independent modes gets quite complicated. To our knowledge the multi-mode REM does not use the phase equation. 

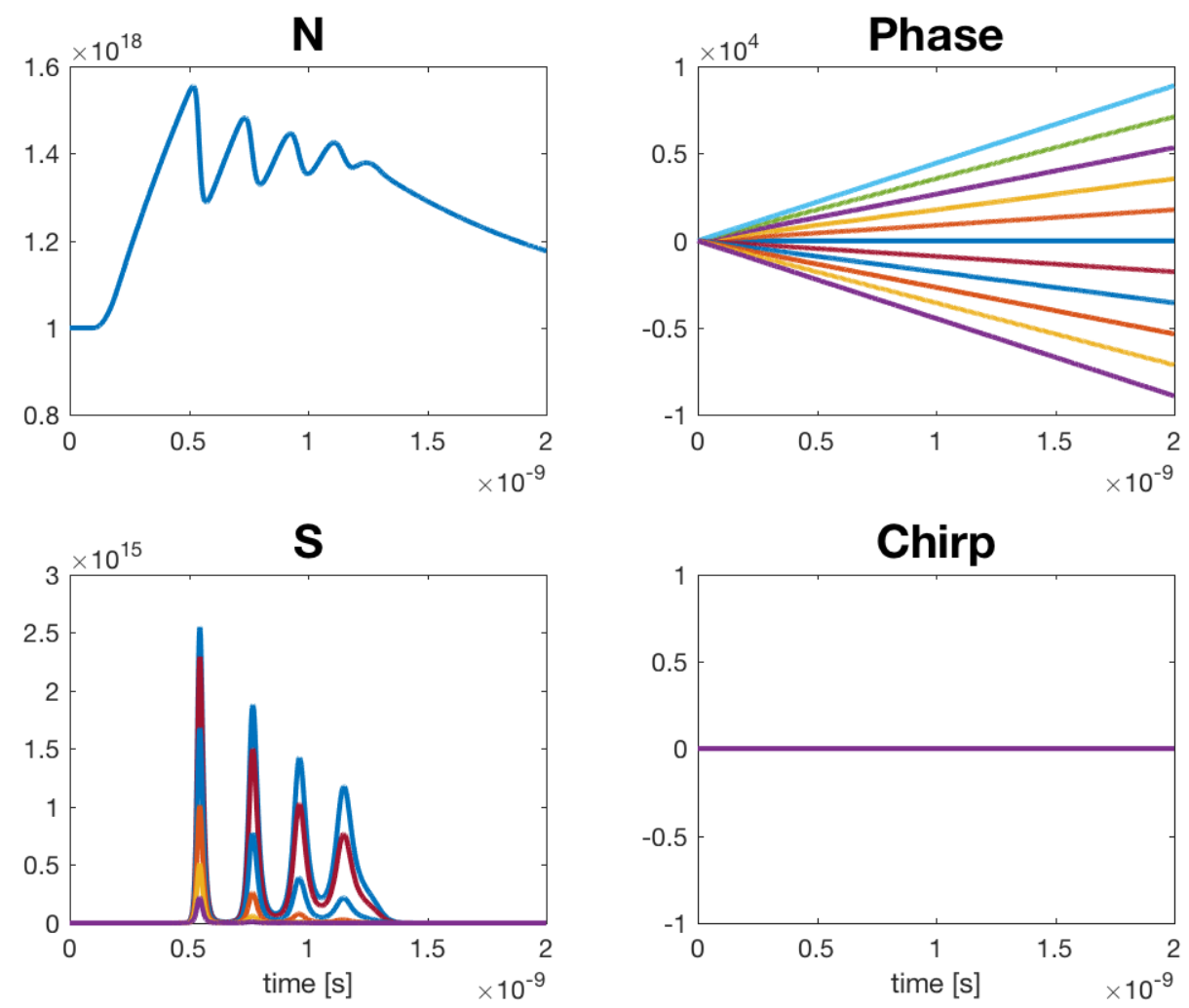

Figure 3.13: Multi-mode REM current pulse simulation, $N$ starting at $N_{t r}$ for 11-mode simulation. $N$ and $S$ have units $\mathrm{cm}^{-3}$ while phase is in radians and chirp rad/s.

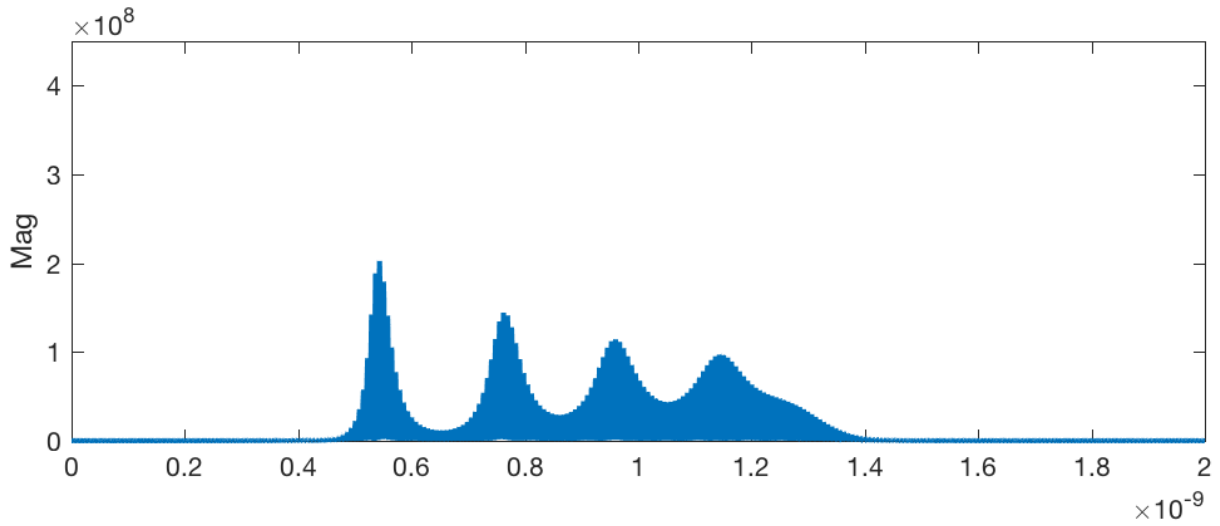

Figure 3.14: Multi-mode REM current pulse simulation, showing $E$ magnitude for 3-mode simulation. 


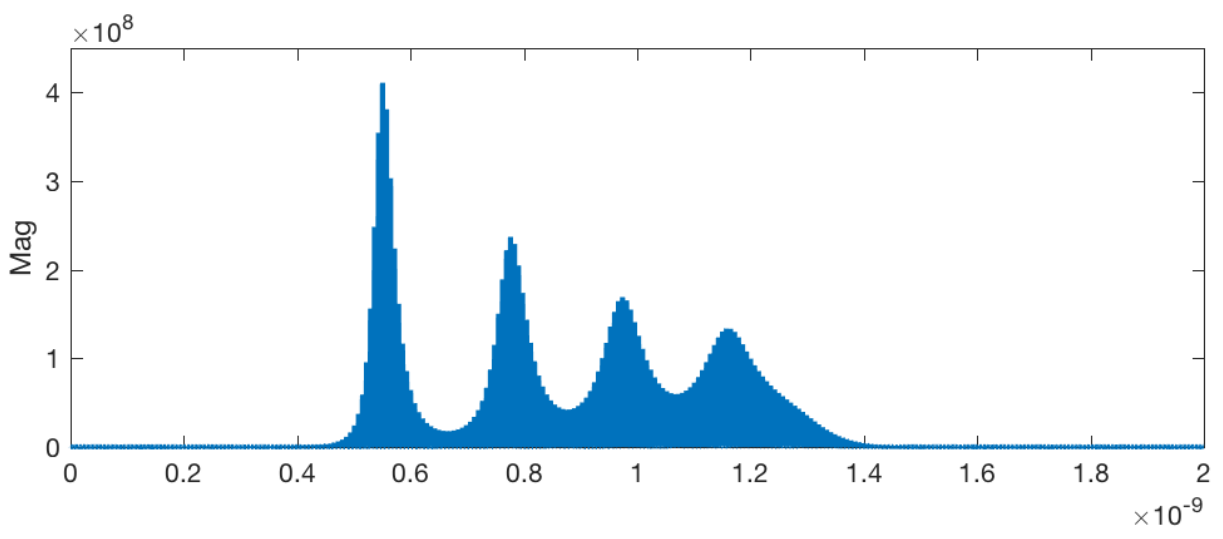

Figure 3.15: Multi-mode REM current pulse simulation, showing $E$ magnitude for 31-mode simulations. 

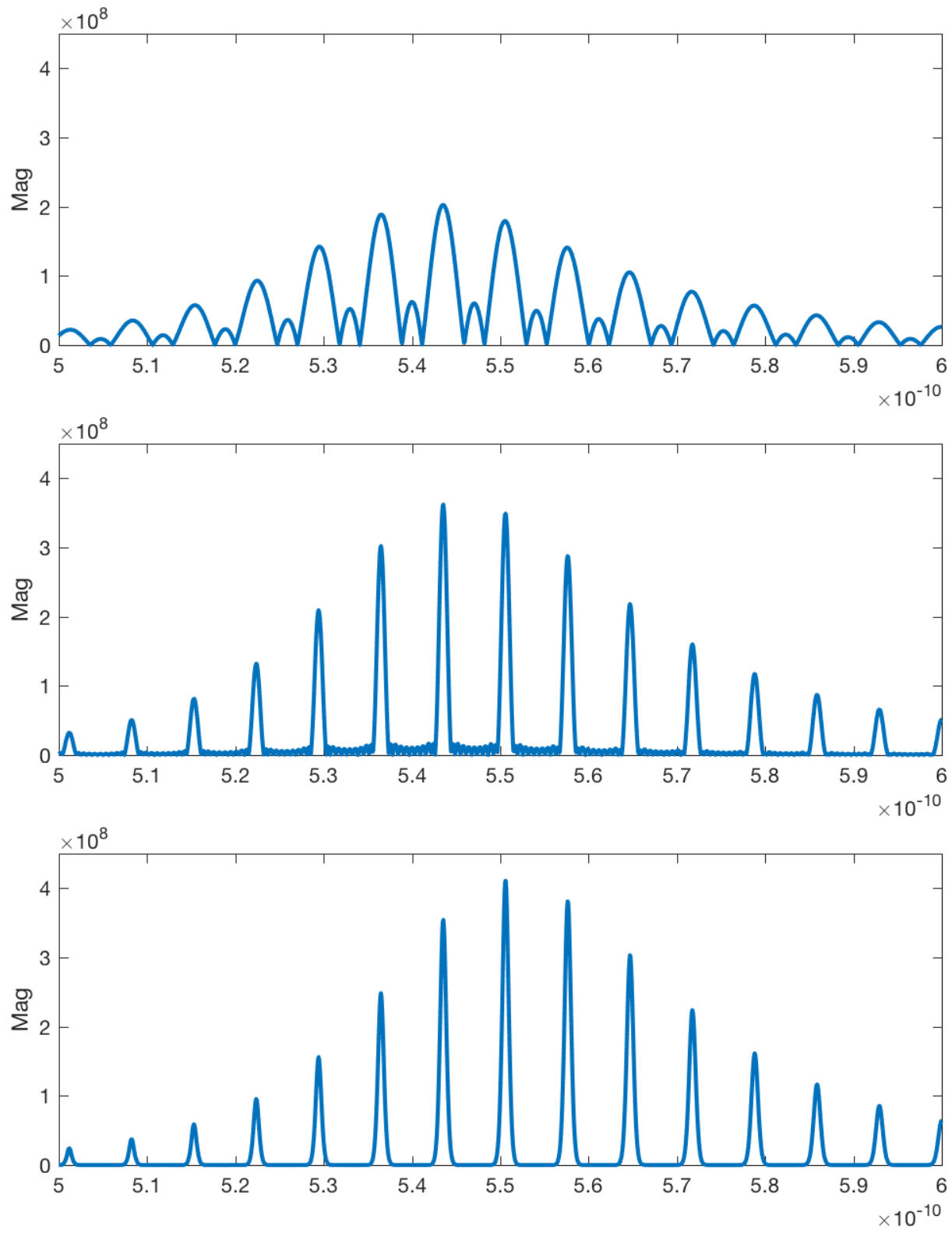

Figure 3.16: Multi-mode REM current pulse simulation, showing zoom of $E$ magnitude for 11 mode simulation without a random phase. Pulses grow and get extremely narrow as number of modes increase. 

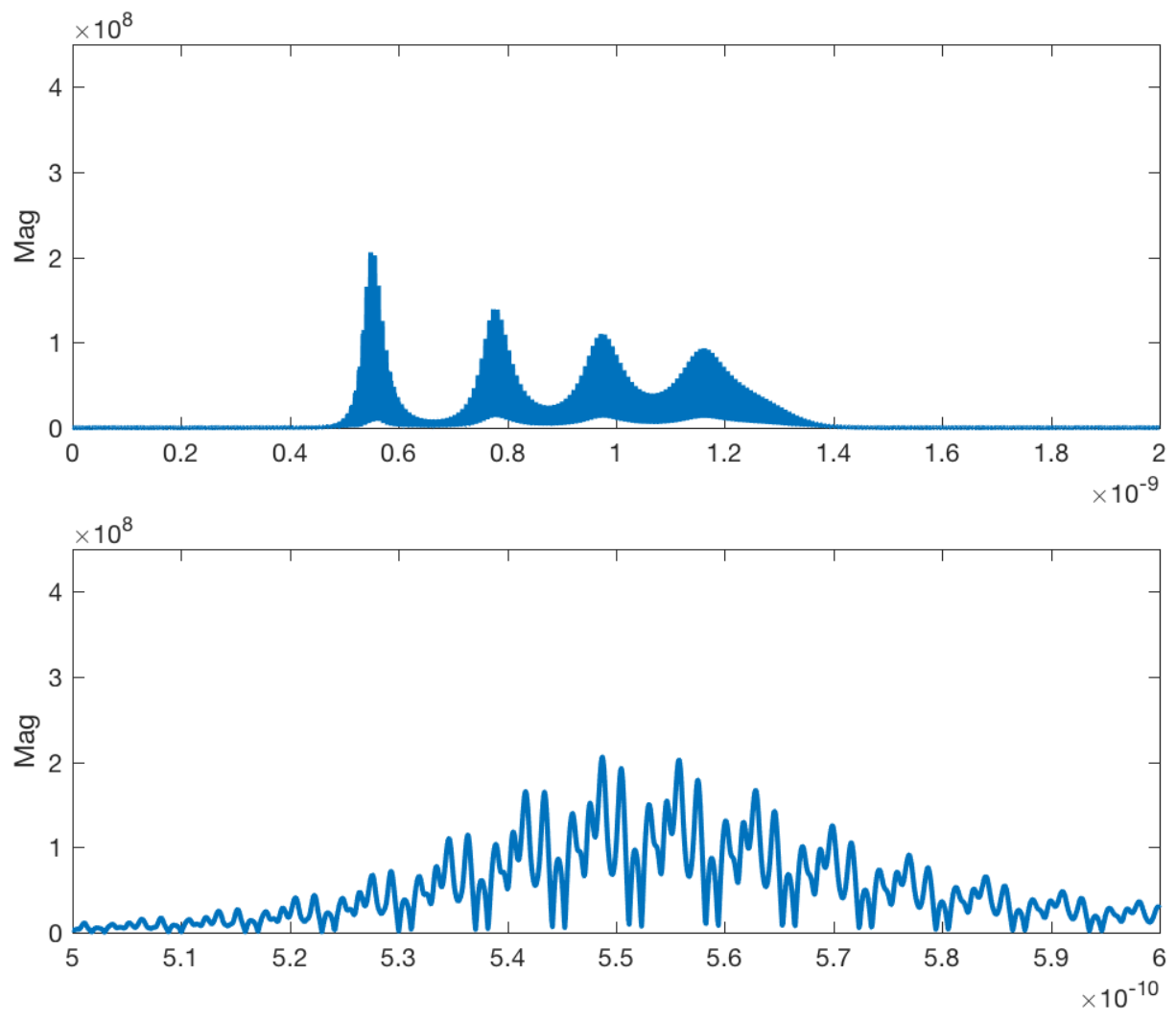

Figure 3.17: Multi-mode REM current pulse simulation, showing $E$ magnitude for 31 mode simulation starting each mode with a random phase. Bottom is a zoom in of top. 


\subsection{Transmission Line Model Fundamentals}

Unlike the REM the Transmission Line Model (TLM) is a distributed model, meaning it calculates model variables over at least one of the physical dimensions. In this case it is the length of the laser - the $z$ direction. This model is based on electrical circuit elements in a configuration that is commonly used to model transmission lines. See figure 3.18. This element represents a slice of the $z$ axis and is replicated along the $z$ dimension for the length of the laser. It effectively implements the second-order wave equation with the $L$ and $C$ circuit elements. This model must be integrated with the carrier equation of the REM to form a laser model whose gain is a function of the carrier density $N$. An advantage of this model is that it integrates well with electronic circuit simulators because it is implemented as a circuit. All of its components already exist in these simulators so there is no new modeling techniques required. It does, however, have some serious drawbacks.

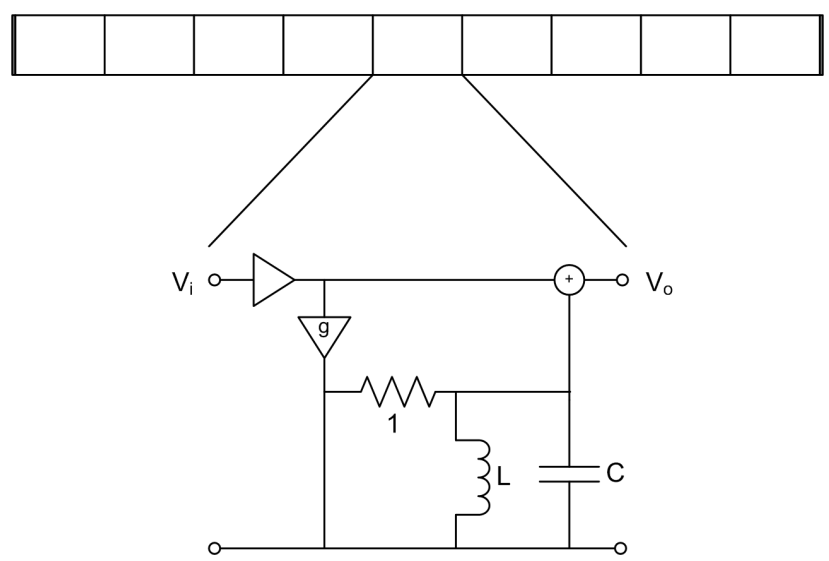

Figure 3.18: TLM model block diagram showing circuit elements.

A disadvantage of this model is that it does not use a complex envelope to give us amplitude and phase of the optical signal. The optical output is the field intensity or photon density and is a real value. Being the same as the electrical blocks its outputs only contain the amplitude of the system variables. This is because the carrier is not part of the signal processing which of course is done to reduce the time step. This means that if a constant modulation signal was compared with another constant modulation from another block, with only a real output (not complex) there is no way that the two signals can interfere with each other even if the carriers are out of phase. Refer back to section 3.1 for a review of the complex envelope. The model does not handle optical signal interference.

This model had an interesting approach to implementing the dispersion relation. It can be seen from the model 'slice' that the frequency response will be determined by the filtering action of each slice. In the figure there are two paths to the output and only the path with gain is filtering. This is called gain dispersion where only the part of the signal with gain is filtered.

The optical spectrum of light emitted from a laser is often modeled as a Lorentzian. A 
Lorentzian is a complex function of frequency that has a peak at a specific frequency. See (2.37) and figure 2.15 for a corresponding plot. This is straightforward for the TLM because Lorentzians can be implemented by an $L C$ filter circuit.

The problem with doing this with optical circuits is that the frequencies involved are too high for an electronic simulator. To solve this problem the optical signals can be shifted down to a lower frequency. For example the carrier frequency $\omega_{c}$ is shifted to a lower circuit frequency $\omega_{t}$ which is easily handled by the simulator. Since the modulation frequency is within the circuit operating frequencies this eliminates the problem of optical frequencies. Unfortunately it creates another. The carrier cannot be shifted to baseband (DC) because it has to be implemented with an $L C$ filter so must have a positive frequency. And then the modulation must be extracted from the new carrier $\left(\omega_{t}\right)$. This is not simple to do. This model has already been implemented elsewhere [14] as a standalone laser simulator but was deemed too difficult to integrate with the MNA simulation engine. The goal was to find a better model for integration based on complex envelopes.

\subsection{Traveling Wave Model Fundamentals}

Like the TLM the TWM is a distributed model, meaning it calculates model variables over the length of the laser, the $z$ direction. Figure 3.19 shows the discretization in $z$. The model permits parameters to be specified for each slice and of course has a value of $N$ and $S$ for each of them. The TWM is, as its name suggests, based on traveling waves which is the fundamental nature of electromagnetic waves. For some simple cases we can presume to know the direction of the radiation but a general model will have to propagate waves in both directions. To do this the forward and reverse direction must be handled separately and then combined to see the total light output. We ignore waves in any other direction. This is valid in a laser as once the photon density has built up via stimulated emission the other directions will be negligible. To obtain the intensity from the electric field is a straightforward calculation. In our semiconductor lasers the magnetic field will not be necessary to calculate and the boundary conditions will become critically important. The model will also presume one carrier frequency, one index of refraction, one group velocity and also assumed one transverse mode. This could be changed if desired. For more information on modes refer to section 2.1.3. The model uses the Slowly Varying Envelope Approximation (SVEA) so that only the carrier envelope is propagated through the model. See appendix $\mathrm{G}$ for more details of the SVEA and the TWM. For lasers the $S$ value must be calculated before it is integrated with the $N$ equation of the classic REM. As with the other models we can calculate power, chirp and other model quantities from the basic waves. There must be a significant advantage provided by the TWM if we are to introduce this added complexity

The main advantage of the TWM is that it is a physical model that naturally arises from Maxwell's equations. The waves are modeled as they would be in 3-D space and so can be visualized. Because of the physical nature of the model the modes are a natural consequence of the distributed waves with boundary conditions and interference. This is not as straightforward as one might think and is explored in A.6. It is expected that many 


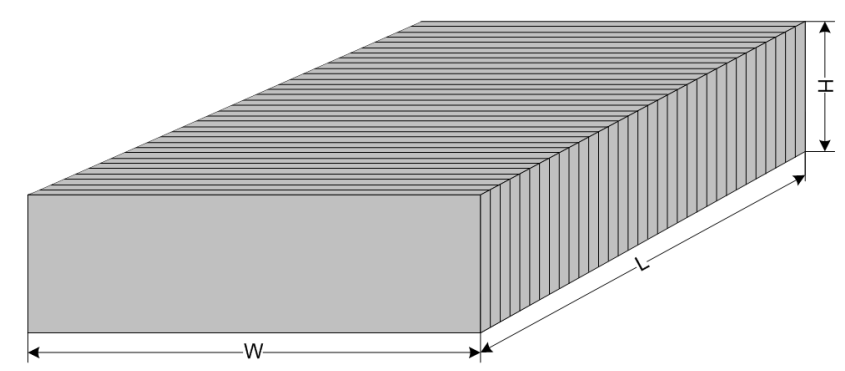

Figure 3.19: Active layer showing discretization of $z$ (along length).

properties will be handled correctly without prior knowledge because the model is physically based.

It was determined that this model needed to be investigated further to verify that it would be a significant improvement over the REM without too severe a penalty in simulation time. The TWM will be discussed in much more detail in chapters 5, 6 and 7 . 


\section{Chapter 4}

\section{Circuit Modeling With Laser Elements}

The main goal of this thesis is to determine if the TWM will be suitable to integrate with the current circuit simulators used today. We first look at the basic implementation of the SPICE type of simulators and then review how well the models we are reviewing integrate with it.

\subsection{Modified Nodal Analysis Overview}

Nodal analysis is a method of generating a system of equations that represent a circuit. Many sources are available on this topic such as [22] [23] [24]. It is usually referred to as Modified Nodal Analysis (MNA). The system of equations will be represented by a matrix of the form

$$
(\boldsymbol{G}+s \boldsymbol{C}) \boldsymbol{x}=\boldsymbol{b}
$$

where $\boldsymbol{G}$ is a square matrix of conductances, $\boldsymbol{C}$ is a square matrix of capacitances, $\boldsymbol{x}$ is a vector of node voltages or currents, and $\boldsymbol{b}$ is a vector of sources. 's' is the complex frequency variable. The system of equations are based on KCL nodal analysis. Each row of the matrix or vector corresponds to a node and would be the KCL equation for that node. As the netlist for the circuit is analyzed each node is defined and labeled. The purpose of the matrix and vectors is to systemize the KCL analysis so that it is easily performed by a digital computer from a netlist of nodes and components. Each type of circuit element such as passive components and sources always take the same form in the matrices relative to their respective nodes. They are known as 'stamps' and given the component with its nodes the 'stamp' can easily be inserted into the matrix. Once the system of matrices is generated it is straightforward for a computer to solve it and provide a solution to the circuit. Many special techniques have been applied to this problem making it increasingly efficient. It is the desire to harness this power for optical circuits that drives the integration with it.

This is best explained through the use of simple examples. An $R C$ circuit is shown in 
figure 4.1. The nodes are arbitrarily numbered (circled numbers) except for the reference node or ground - this is always node number 0 . This circuit has 3 nodes with voltages $V_{1}, V_{2}$, and $V_{3}$. The resistors are labeled as conductances - this simplifies the matrix when dealing with currents as we don't have to show all of the resistances inverted. Let's start with the

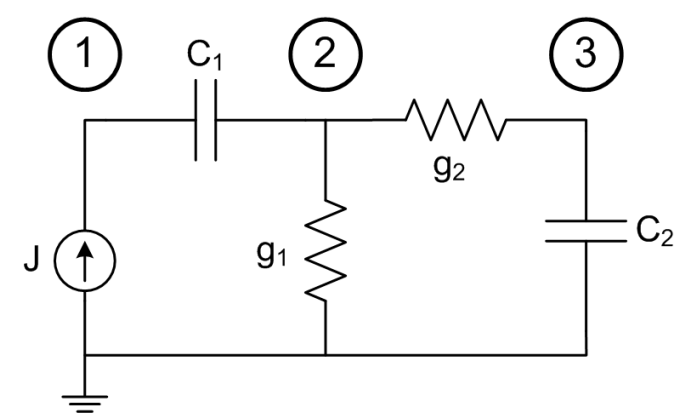

Figure 4.1: Simple $R C$ circuit. Resistors shown as conductances. Nodes numbered within circles.

stamp for a resistor, shown in figure 4.2. This shows the general case where the resistor is placed between two nodes numbered $i$ and $j$. Figure 4.3 shows the special case where one of the nodes is the reference node. This stamp is simpler as any node that is connected to ground or node 0 is simply omitted. Note the symmetry of the 'stamps' through the diagonal. This turns out to be extremely helpful in solving the equations efficiently.

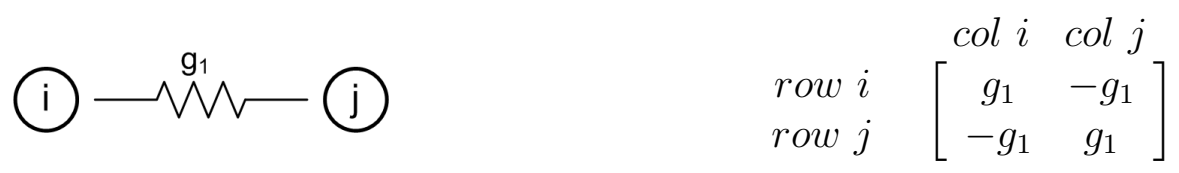

Figure 4.2: Resistor with labeled nodes and corresponding 'stamp'.
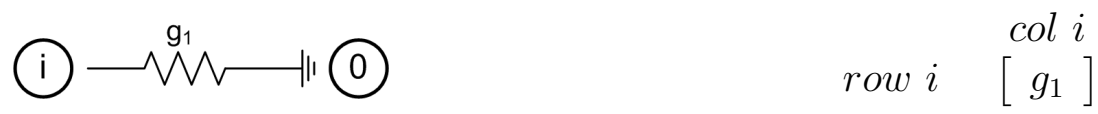

Figure 4.3: Resistor with labeled nodes and corresponding 'stamp' when one node is reference node 0 .

To understand the 'stamps' let's examine the KCL equations and compare them to the 'stamp'. Figure 4.4 shows a resistor with one node connected to ground and a current source $J$ into this node. KCL says that the sum of currents at a node sum to 0 . At node 1 we have the current into the node from the source and the current out through the resistor which equal

$$
I_{\text {in }}=J \quad I_{\text {out }}=\left(V_{i}-0\right) g=V_{i} g
$$

Setting these equal we get

$$
J=V_{i} g
$$




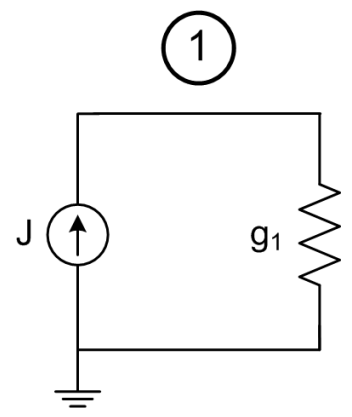

Figure 4.4: Resistor connected to current source. Resistor shown as conductance. Node numbered within circle.

which of course we could have written down by inspection. To write this in matrix form of (4.1) and leaving the $C$ matrix 0 for now, we get

$$
\begin{aligned}
\boldsymbol{G x} & =\boldsymbol{b} \\
{[g]\left[V_{i}\right] } & =[J]
\end{aligned}
$$

Note the size of the $G$ matrix is equal to the number of nodes not counting the reference node. This corresponds to the stamp of figure 4.3. Figure 4.5 shows the more general case where the resistor stamp connects to two nodes: two resistors in series to ground connected to the current source. From KCL at node 1 we get

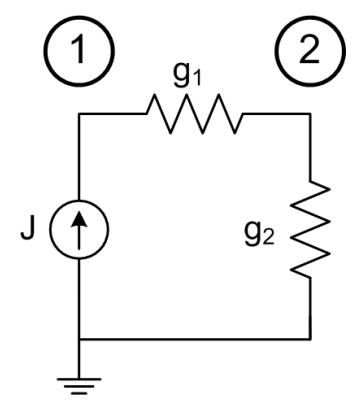

Figure 4.5: Resistors connected in series to current source. Resistors shown as conductances. Nodes numbered within circles.

$$
J_{1}=\left(V_{1}-V_{2}\right) g_{1}
$$

and at node 2 we get

$$
\left(V_{1}-V_{2}\right) g_{1}=V_{2} g_{2}
$$

If we rearrange the terms with the voltages in the same order and sources on the right we get

$$
\begin{aligned}
g_{1} V_{1}-g_{1} V_{2} & =J_{1} \\
-g_{1} V_{1}+\left(g_{1}+g_{2}\right) V_{2} & =0
\end{aligned}
$$


Now rewriting this in matrix form we get

$$
\begin{aligned}
\boldsymbol{G x} & =\boldsymbol{b} \\
{\left[\begin{array}{cc}
g_{1} & -g_{1} \\
-g_{1} & g_{1}+g_{2}
\end{array}\right]\left[\begin{array}{c}
V_{1} \\
V_{2}
\end{array}\right] } & =\left[\begin{array}{c}
J_{1} \\
0
\end{array}\right]
\end{aligned}
$$

The concept of a 'stamp' is more obvious. The first resistor with conductance $g_{1}$ appears in the $i$ th and $j$ th rows and columns with a + or - sign as shown in the resistor stamp in figure 4.2. $g_{2}$ as with our first example of a node connected to the reference only appears in the $j$ th row and column. It can also be seen that when conductances appear in the same matrix element they are simply added. There is also a 'stamp' for a current source as shown in figure 4.6. As with the resistor 'stamp' if one of the nodes is the reference node the entries
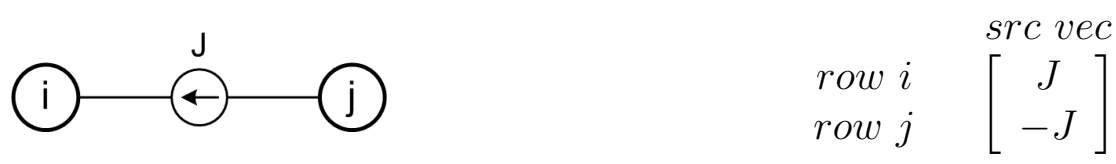

Figure 4.6: Current source with labeled nodes and corresponding 'stamp'. Note that this stamp is applied to the source vector $\boldsymbol{b}$. If one of the nodes is the reference node leave it empty.

for that row/column are left empty. In the two resistor example (4.8) we see that the current stamp is also valid for this example because the $j$ th node was the reference node.

The nodal analysis is a systematic way of generating the KCL system of equations for a circuit netlist in matrix form. The matrix equation is then easily solved by computers by various methods that have been developed and optimized. Each node of the circuit is then calculated and circuit analysis is easily accomplished by a digital computer.

We have looked at the current source. It turns out to be simple in the KCL analysis. The voltage source is a bit more complicated. If we connect a resistor to a voltage source $E$ and ground (figure 4.7) KCL at node 1 says

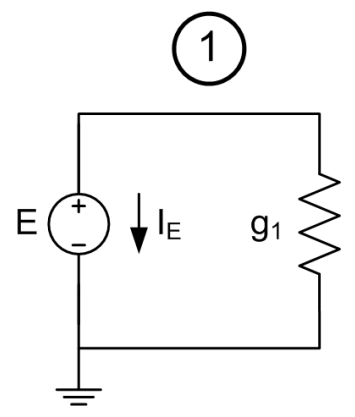

Figure 4.7: Resistor connected in series to voltage source.

$$
-I_{E}=V_{1} g
$$


where we have defined $I_{E}$ as current flowing in to the source $E$ from the resistor. We must also specify

$$
V_{1}=E
$$

so we have two equations where as before for the current source we only had one. Writing these in our matrix formulation we have

$$
\begin{aligned}
\boldsymbol{G x} & =\boldsymbol{b} \\
{\left[\begin{array}{cc}
g_{1} & 1 \\
1 & 0
\end{array}\right]\left[\begin{array}{c}
V_{1} \\
I_{E}
\end{array}\right] } & =\left[\begin{array}{c}
0 \\
E
\end{array}\right]
\end{aligned}
$$

We have added a new variable $I_{E}$ to our system of equations which results in an additional row and column. As with the other stamps if the second node is not connected to the reference node we end up with -1 in the row/column for that node. The stamp then is as shown in figure 4.8 .
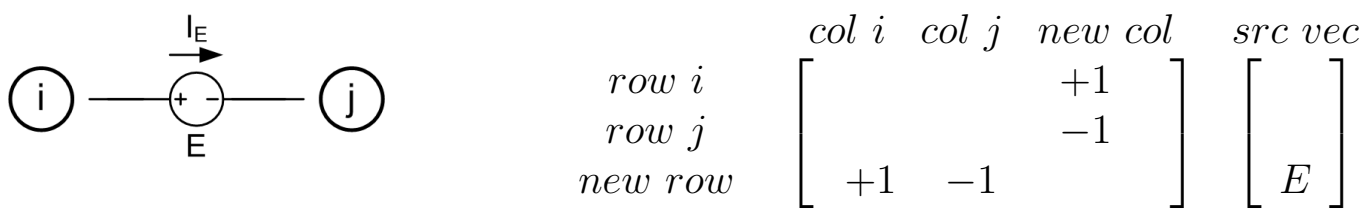

Figure 4.8: Voltage source with labeled nodes and corresponding stamp. Note that this stamp is applied to both the $\boldsymbol{G}$ matrix and the source vector $\boldsymbol{b}$. A new column and row are added representing the source current. If one of the nodes is the reference node leave it empty.

If we look at (4.1) we see that there is a second matrix $C$ which has not been used yet. This matrix is used for reactive components $C$ and $L$. Let us first take a look at the capacitor stamp. The matrix locations and signs are identical to the resistor stamp - there is just the complex frequency factor multiplying each term. And like the other stamps, if one of the nodes is the reference node the corresponding rows/columns are left empty. Let's take a

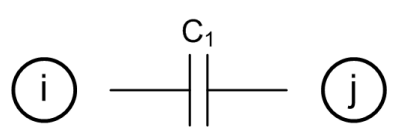

$$
\begin{aligned}
& \text { col } i \quad c o l j \\
& \begin{array}{l}
\text { row } i \\
\text { row } j
\end{array} \quad\left[\begin{array}{cc}
s C_{1} & -s C_{1} \\
-s C_{1} & s C_{1}
\end{array}\right]
\end{aligned}
$$

Figure 4.9: Capacitor with labeled nodes and corresponding 'stamp'. If one of the nodes is the reference node leave its rows/columns empty.

simple example of a source connected through a resistor to a capacitor with the other end grounded. This is shown in figure 4.10. Node 1 is the voltage source output and node 2 is the capacitor voltage. With our stamp rules we can write the matrix equation by inspection. 


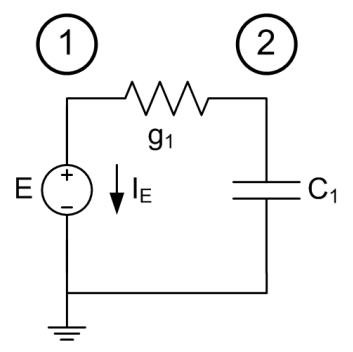

Figure 4.10: Simple $R C$ filter circuit.

The voltage source is connected to the reference node so the -1 terms are left out. We see that the capacitor has been treated like a resistor with conductance $s C_{1}$.

$$
\left[\begin{array}{ccc}
g_{1} & -g_{1} & 1 \\
-g_{1} & g_{1}+s C_{1} & \\
1 & &
\end{array}\right]\left[\begin{array}{c}
V_{1} \\
V_{2} \\
I_{E}
\end{array}\right]=\left[\begin{array}{c}
0 \\
0 \\
E
\end{array}\right]
$$

Because the reactive components are associated with the complex frequency variable $s$ we will separate them out into the $\boldsymbol{C}$ matrix. It is similar to the $\boldsymbol{G}$ matrix in the equation but we know the reactive components have different dynamics in a circuit. Rewriting (4.12) by seperating the $s$ terms we get

$$
\begin{gathered}
(\boldsymbol{G}+s \boldsymbol{C}) \boldsymbol{x}=\boldsymbol{b} \\
\left(\left[\begin{array}{ccc}
g_{1} & -g_{1} & 1 \\
-g_{1} & g_{1} & \\
1 & &
\end{array}\right]+s\left[\begin{array}{ccc}
0 & 0 & 0 \\
0 & C_{1} & 0 \\
0 & 0 & 0
\end{array}\right]\right)\left[\begin{array}{c}
V_{1} \\
V_{2} \\
I_{E}
\end{array}\right]=\left[\begin{array}{c}
0 \\
0 \\
E
\end{array}\right]
\end{gathered}
$$

The node analysis stamps are useful for algorithmically solving circuits with digital computers given a netlist of components. The netist is converted to a system of equations which are readily solved to give the DC analysis of the circuit. If there are capacitors we see that the circuit will be solved for one frequency where the reactance of the capacitor is calculated for that frequency and used as its resistance. It is easy to extend this to give a frequency response because the complex frequency variable can be swept through any range and the values of each node can be calculated.

Because the complex frequency variable $s$ appears in the matrix equation this appears to be a problem for inductors because the complex frequency variable will appear in the denominator. What would happen if we wanted to sweep to DC?

To avoid these problems inductors have a more complicated stamp than capacitors. Taking a simple $L C$ circuit with a current source driving an inductor connected through a capacitor to ground we develop the inductor stamp. The first step is to treat the inductor like we did the resistor and capacitor. The matrix equation is

$$
\left[\begin{array}{cc}
\frac{1}{s L} & -\frac{1}{s L} \\
-\frac{1}{s L} & \frac{1}{s L}+s C
\end{array}\right]\left[\begin{array}{l}
V_{1} \\
V_{2}
\end{array}\right]=\left[\begin{array}{l}
J \\
0
\end{array}\right]
$$


This cannot be put into the form of (4.1). Let's introduce another variable $I_{L}$ which is the current through the inductor

$$
I_{L}=\frac{1}{s L}\left(V_{1}-V_{2}\right)
$$

and rearrange

$$
V_{1}-V_{2}-s L I_{L}=0
$$

which does not have $s$ in the denominator. If we write out the matrix equation as a system of equations:

$$
\begin{aligned}
\frac{1}{s L}\left(V_{1}-V_{2}\right) & =J \\
\frac{1}{s L}\left(V_{2}-V_{1}\right)+s C V_{2} & =0
\end{aligned}
$$

Here we can substitute in $I_{L}$ to get three equations

$$
\begin{aligned}
I_{L} & =J \\
-I_{L}+s C V_{2} & =0 \\
V_{1}-V_{2}-s L I_{L} & =0
\end{aligned}
$$

which in matrix form is

$$
\left[\begin{array}{ccc}
0 & 0 & 1 \\
0 & s C & -1 \\
1 & -1 & -s L
\end{array}\right]\left[\begin{array}{l}
V_{1} \\
V_{2} \\
I_{L}
\end{array}\right]=\left[\begin{array}{l}
J \\
0 \\
0
\end{array}\right]
$$

This is now in the correct form. Because we have no resistors in this circuit this matrix is the $C$ matrix. This gives us the inductor stamp as shown in figure 4.11. The frequency
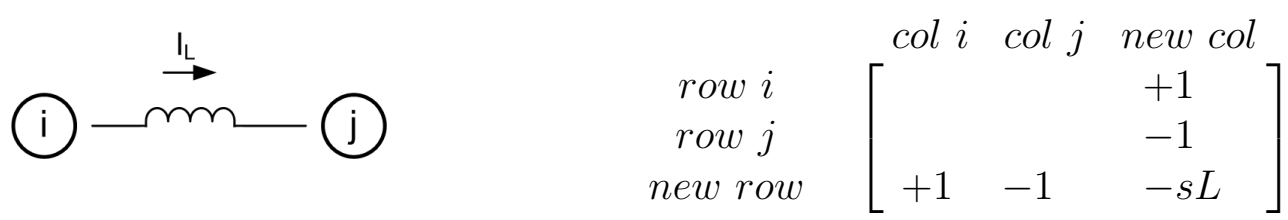

Figure 4.11: Inductor with labeled nodes and corresponding stamp. A new column and row are added representing the inductor current. If one of the nodes is the reference node leave it empty.

response can be calculated as it was for the capacitor case.

There are many other stamps that have been developed for nodal analysis for elements including voltage and current controlled sources, short circuits, and many others. We will 
not show anymore but they can be readily obtained from many sources including [24]. Using these basic stamps more complex models can be generated for operational amplifiers and transistors to name just two.

This nodal analysis technique is usually referred to as Modified Nodal Analysis (MNA) and can also be used to solve circuits in the time domain. Starting with (4.1)

$$
(\boldsymbol{G}+s \boldsymbol{C}) \boldsymbol{x}=\boldsymbol{b}
$$

we can bring this equation into the time domain. Given that multiplying by $s$ in the frequency domain is equivalent to taking the derivative in the time domain we have

$$
\boldsymbol{G} x(t)+\boldsymbol{C} \dot{\boldsymbol{x}}(t)=\boldsymbol{b}(t)
$$

This is a first-order DE and can be solved using FDTD techniques. The MNA stamps combined with frequency analysis and time domain analysis form the basis of the modern day SPICE type of circuit simulators. Much study has been done on topics such as stability, order reduction but this is not required for this thesis.

The last element we will consider is the nonlinear circuit element. This element will not fit into the simple $G$-matrix equation of (4.1). From the circuit in figure 4.1 we can write

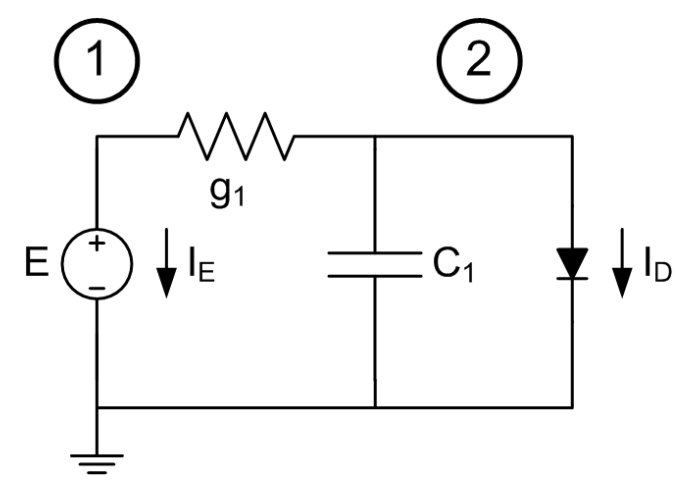

Figure 4.12: Example nonlinear circuit.

down the MNA equations directly from (4.12). We still use KCL to generate the system of equations at each node but we add a term to include the nonlinear diode component.

$$
\left[\begin{array}{ccc}
g_{1} & -g_{1} & 1 \\
-g_{1} & g_{1} & \\
1 & &
\end{array}\right]\left[\begin{array}{c}
V_{1} \\
V_{2} \\
I_{E}
\end{array}\right]+\left[\begin{array}{ccc}
0 & 0 & 0 \\
0 & C_{1} & 0 \\
0 & 0 & 0
\end{array}\right]\left[\begin{array}{c}
\dot{V}_{1} \\
\dot{V}_{2} \\
\dot{I}_{E}
\end{array}\right]+\left[\begin{array}{c}
0 \\
I_{D} \\
0
\end{array}\right]=\left[\begin{array}{c}
0 \\
0 \\
E
\end{array}\right]
$$

This new vector becomes our nonlinear vector $\boldsymbol{f}(t)$. This vector can be a function of any nodes and we have

$$
\boldsymbol{G} x(t)+\boldsymbol{C} \dot{\boldsymbol{x}}(t)+\boldsymbol{f}(t)=\boldsymbol{b}(t)
$$

which is a nonlinear system of algebraic equations. These can be solved with initial conditions and Newton-Raphson iterations. 
Let's first look at the simplest nonlinear element - a nonlinear resistor. As with a linear resistor the current adds to the rows of both nodes, but instead of only being a function of its two nodes it is a nonlinear function of all variables so appears in the nonlinear $\boldsymbol{f}$ vector. See figure 4.13.

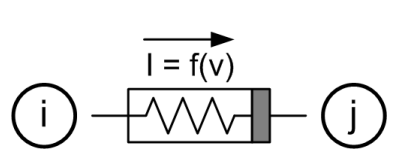

$$
\text { rowi }\left[\begin{array}{c}
f(v) \\
-f(v)
\end{array}\right]
$$

Figure 4.13: Nonlinear resistor with labeled nodes and corresponding stamp. Note that this stamp is applied to the nonlinear $\boldsymbol{f}$ vector. If one of the nodes is the reference node leave it empty.

The nonlinear capacitor stamp is more complicated as we have to add a new variable. First examine figure 4.14. We've added the variable $Q$, the charge, because we know it is a

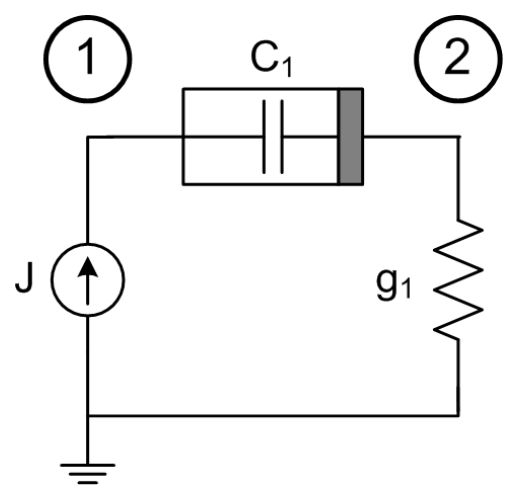

Figure 4.14: Example nonlinear capacitor circuit.

function of the capacitor voltage which will now be nonlinear. The current is simply $d Q / d t$ so we have from KCL the set of equations

$$
\begin{array}{r}
\frac{d Q}{d t}=J \\
-\frac{d Q}{d t}+g_{1} V_{2}=0 \\
Q=f\left(V_{1}-V_{2}\right)
\end{array}
$$

which can be written in our matrix notation as

$$
\left[\begin{array}{ccc}
0 & 0 & 0 \\
0 & g_{1} & 0 \\
0 & 0 & 1
\end{array}\right]\left[\begin{array}{l}
V_{1} \\
V_{2} \\
Q
\end{array}\right]+\left[\begin{array}{ccc}
0 & 0 & 1 \\
0 & 0 & -1 \\
0 & 0 & 0
\end{array}\right]\left[\begin{array}{c}
\dot{V}_{1} \\
\dot{V}_{2} \\
\dot{Q}
\end{array}\right]+\left[\begin{array}{c}
0 \\
0 \\
f\left(V_{1}-V_{2}\right)
\end{array}\right]=\left[\begin{array}{l}
J \\
0 \\
0
\end{array}\right]
$$



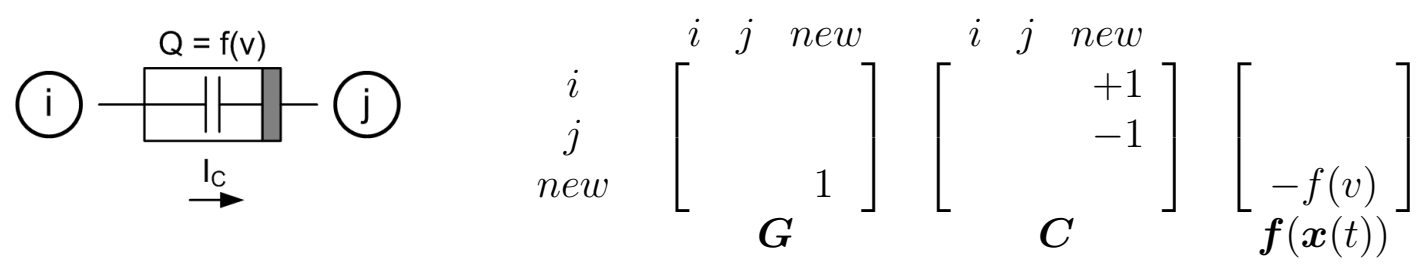

Figure 4.15: Nonlinear capacitor with labeled nodes and corresponding stamp. Note that this stamp is applied to the $\boldsymbol{G}$ and $\boldsymbol{C}$ matrices as well as the nonlinear $\boldsymbol{f}$ vector. If one of the nodes is the reference node leave it empty.

with a new row and column for the new variable $Q$. See figure 4.15 for the stamp.

Once the nonlinear vector is added to the matrix equation it can no longer be solved directly as in the linear circuit case. The procedure is to take an initial guess and use Newton-Raphson iterations.

To see how the Newton-Raphson iterations work let's take a look at finding the initial DC solution for a nonlinear circuit. We have the nonlinear matrix equation (4.25) from which we can eliminate the $\boldsymbol{C}$ matrix for the DC solution

$$
\boldsymbol{G} \boldsymbol{x}+\boldsymbol{f}(\boldsymbol{x})=\boldsymbol{b}
$$

and change the form to

$$
\boldsymbol{\Phi}(\boldsymbol{x})=\boldsymbol{G} \boldsymbol{x}+\boldsymbol{f}(\boldsymbol{x})-\boldsymbol{b}=0
$$

and solve for $\boldsymbol{x}$ which is a vector.

Newton-Raphson iterations work on the idea that for any function, if $x_{0}$ is a good estimate for a root then a better estimate is

$$
x_{1}=x_{0}-\frac{g\left(x_{0}\right)}{g^{\prime}\left(x_{0}\right)}
$$

To apply this to $\boldsymbol{\Phi}(\boldsymbol{x})$ we need the derivative of $\boldsymbol{\Phi}(\boldsymbol{x})$. Remember that $\boldsymbol{x}$ is a vector. The derivative is called the Jacobian $\boldsymbol{J}$

$$
\boldsymbol{J}(\boldsymbol{x})=\frac{d \boldsymbol{\Phi}(\boldsymbol{x})}{d \boldsymbol{x}}=\boldsymbol{G}+\frac{d \boldsymbol{f}(\boldsymbol{x})}{d \boldsymbol{x}}
$$

From this we see that we will need the derivatives of all the nonlinear functions with respect to all inputs.

$$
\boldsymbol{f}(\boldsymbol{x})=\left[\begin{array}{c}
f_{1} \\
f_{2} \\
\vdots \\
f_{n}
\end{array}\right]
$$




$$
\frac{d \boldsymbol{f}(\boldsymbol{x})}{d \boldsymbol{x}}=\left[\begin{array}{cccc}
\frac{\partial f_{1}}{\partial x_{1}} & \frac{\partial f_{1}}{\partial x_{2}} & \ldots & \frac{\partial f_{1}}{\partial x_{n}} \\
\frac{\partial f_{2}}{\partial x_{1}} & \frac{\partial f_{2}}{\partial x_{2}} & \cdots & \frac{\partial f_{2}}{\partial x_{n}} \\
\vdots & \vdots & \ddots & \vdots \\
\frac{\partial f_{n}}{\partial x_{1}} & \frac{\partial f_{n}}{\partial x_{2}} & \cdots & \frac{\partial f_{n}}{\partial x_{n}}
\end{array}\right]
$$

Once we have the Jacobian we can do Newton-Raphson iterations. Start with an initial guess $\boldsymbol{x}^{(0)}$ then calculate the next estimate

$$
\boldsymbol{x}^{(1)}=-\boldsymbol{J}^{-1}\left(\boldsymbol{x}^{(0)}\right) \boldsymbol{\Phi}\left(\boldsymbol{x}^{(0)}\right)
$$

where $\boldsymbol{J}^{-1}\left(\boldsymbol{x}^{(0)}\right)$ is the inverse of the Jacobian evaluated at $\boldsymbol{x}^{(0)}$. This should converge and give us our solution for $\boldsymbol{x}$.

A similar approach is used while time stepping through the nonlinear circuit.

This brief overview of the MNA engine within SPICE-like simulators shows how the basics of how the engine works. It is the systematic forming of matrices of circuit elements with stamps and then using special matrix techniques easily handled by digital computers to solve for all of the nodes in the system in frequency or in time. There is also facility to handle nonlinear components which is critical to our optical circuit compact models. Refer back to figure 2.21 for an example of a nonlinear compact circuit model.

\subsection{Integration with Modified Nodal Analysis Simula- tion Engine}

The goal was to integrate the models discussed in this thesis with the OptiSPICE MNA simulation engine. The MNA simulation engine is an implicit multi-domain simulator that handles electrical, optical and thermal domains simultaneously. Being implicit it is extremely fast at the expense of large data structures. All circuit elements are instantiated as stamps as described in section 4.1. Even compact models with nonlinear elements are stamped. The widespread use of these simulators makes it desirable to integrate our compact optical models with them.

Optical signals are handled as a complex envelope in the MNA engine. Complex variables are used to track the signal's phase and amplitude without processing the high bandwidth optical carrier. This means the carrier is implicit in this formulation. Because optical signals have direction they must be propagated in both directions separately with forward and reverse waves which are then added to obtain the real output.

In order to integrate with the MNA simulation engine models require the usual circuit stamps with nonlinear equations and the inputs and outputs to connect them into the circuit. The nonlinear aspect also requires derivatives for the Jacobian of all nonlinear components. These derivatives must be with respect to each input and can be difficult to obtain as often the derivatives are not known analytically. Fortunately they can then be calculated numerically by the MNA engine. Being nonlinear, the models under consideration (REM, 
TLM, and TWM) would all be instantiated in the same MNA system context; a nonlinear stamp with derivatives required.

The REM model has a set of nonlinear differential equations that are instantiated as a stamp. A simplified version is shown in figure 2.21. The nonlinear elements are simple analytic expressions so can be included in a module called during the implicit time step solver. They are nonlinear because they are functions of more than one variable.

The TLM is an explicit time stepping model and so even though its components are all found in the simulator it does not integrate directly into the MNA engine which is an implicit FDTD solver. It would be instantiated as a nonlinear stamp and the derivatives with respect to all inputs required. Often if the signals do not change much during a time step they can be assumed to be 0 which simplifies the calculations. If this cannot be assumed the derivatives have to be calculated numerically for each Newton-Raphson iteration. The TLM has a synchronization condition which would require a separate time step for the TLM (discussed later in section 5.6). This would mean a separate explicit simulation would need to be run every system step and the inputs and outputs extrapolated between the two simulations. As mentioned in section 3.7, it did not have a complex envelope implemention and there were difficulties in handling the carrier. For these reasons the TLM was not integrated with the MNA engine. Specific results from this model were done by the standalone TLM simulator and were not a part of this thesis. The difficulty in integrating this model with the MNA engine was the main motivation in pursuing the TWM.

The TWM model uses a complex envelope so this is not a problem. It does not integrate easily with the MNA formulation, however, because it is an explicit time stepping model like the TLM. It was implemented as a nonlinear circuit element which is calculated using standard MNA nonlinear methods utilizing a Jacobian matrix and Newton-Raphson iterations to converge the nonlinear portion to a valid solution. The derivative of the nonlinear function with respect to all input variables is required for the Jacobian. This is not known analytically and so must be calculated for each Newton-Raphson iteration adding much overhead.

The TWM also has the time step synchronization condition (discussed later in section 5.6). Generally the TWM time step is much smaller than the system time step and will not line up with it. Therefore the TWM must be advanced past the required time and then the value at that time must be interpolated. Because the MNA simulator should ensure the changes in the system values are small linear interpolation of system values for the TWM is sufficient. The integration of the TWM with the MNA engine is detailed in [3]. 


\section{Chapter 5}

\section{Traveling Wave Model}

The TWM is a distributed model meaning the optical wave is calculated over space as well as time. In practice it only varies in the longitudinal direction $(z$ is chosen to represent this dimension) and the other two are assumed constant over the physical extents. This is not exact but of course is more accurate than a lumped model which assumes the same value over all three dimensions for each point in time. The transverse modes are not the same for all lasers. This model will assume that a single transverse mode approximation is adequate.

The TWM is well studied and many aspects have been examined. A few references are given for further reading: [25]-[29] A tutorial on TWM was found on the internet. [30]

A simplified version of the traveling wave equations are shown in (5.1). The \pm is for the forward and reverse traveling waves, so there are really two equations. The $\beta$ parameter is defined by several other parameters: static detuning $(\delta)$, static loss $\left(\alpha_{b}\right)$, and the Henry factor $\left(\alpha_{H}\right)$. These will be explained in more detail later.

$$
\begin{gathered}
\left(\frac{\partial}{\partial t} \pm v \frac{\partial}{\partial z}\right) E_{ \pm}(z, t)=-i v \beta E_{ \pm}(z, t) \\
\beta=\frac{1}{2}\left[\delta-i \alpha_{b}+\left(i+\alpha_{H}\right) g\right]
\end{gathered}
$$

Note that the equations are first-order which makes them less computationally costly. The reduction to first-order is done by the SVEA which restricts the modulation frequency to much less than the carrier frequency. This is always a reasonable assumption for a carrier modulation scheme. Using Maxwell's equations and the SVEA the traveling wave equations can be derived and this is done in appendix G.

Once the electric field of the forward and reverse traveling waves are known the photon density can be calculated by adding the waves and then finding the intensity (complex conjugate of the E-field). The complex number representation will give correct results regarding interference and takes into account the carrier phase but must be used correctly when performing system simulations. Refer to section 3.1 and appendix A for more information on the complex envelope properties. Again, as in the other models under consideration the carrier frequency is not part of the E-field representation which greatly reduces the calcuations required. 


\subsection{Simplified Traveling Wave Model}

We take a look at the simplified traveling wave equations first. All terms have been dropped from the right-hand side of the equations. This is done to give a better understanding of the equations by removing some unecessary complications - it is much easier to see that they behave as expected in their simple form. The removed terms will have to be added back in as the model is of limited used in this form. The TWM can be run in isolation from a circuit simulator. We call this mode standalone and use it to investigate the internal workings of the active layer when the surrounding circuitry is not of interest. With the simplified traveling wave equations we can determine some of their basic properties while remaining in the analytic domain. Later we will explore the implementation issues when the equations are discretized.

For these simplified traveling wave equations we are modeling a waveguide. It would be equivalent to a a Semiconductor Optical Amplifier (SOA). There are no mirrors and in the following examples and for clarity we will only propagate waves in the forward direction.

\subsection{Zero $\beta$}

The simplest form of the traveling wave equation is when $\beta$ is zero. This results in a fairly straightforward PDE. Note we have dropped the \pm notation and will only work with forward traveling waves for these simple cases. The same would apply for reverse traveling waves.

$$
\left(\frac{\partial}{\partial t}+v \frac{\partial}{\partial z}\right) E(z, t)=0
$$

We can guess that a solution to this will be a plane wave.

$$
E(z, t)=A_{0} e^{i\left(k_{e} z-\omega_{e} t\right)}
$$

Plugging this into the PDE we get

$$
\begin{gathered}
-i \omega_{e} E(z, t)+i v k_{e} E(z, t)=0 \\
-\omega_{e}+v k_{e}=0 \\
k_{e}=\frac{\omega_{e}}{v}
\end{gathered}
$$

where $v$ is the phase velocity. At the input boundary condition we have

$$
E(0, t)=A_{0} e^{i \omega_{e} t}
$$

where we have assumed $z=0$ at input (left side of active layer). We define $k_{e}$ as the wave vector for the unperturbed traveling wave. This solution is the expected wave equation solution. See Figure 5.1. Note that there is no change in frequency, amplitude or phase. 
Another solution to this PDE is a constant input which is a degenerate case of the plane wave where $\omega_{e}$ and $k_{e}$ are both 0 . The solution is

$$
E(z, t)=A_{0}
$$

Both time and spatial derivatives are 0 so this is also a solution to the PDE. Were we to plot it the input would just propagate with no change in amplitude or phase.

So we see that with zero $\beta$ the TWM will just propagate the envelope without changing the phase, amplitude or frequency. We call this simple propagation. 

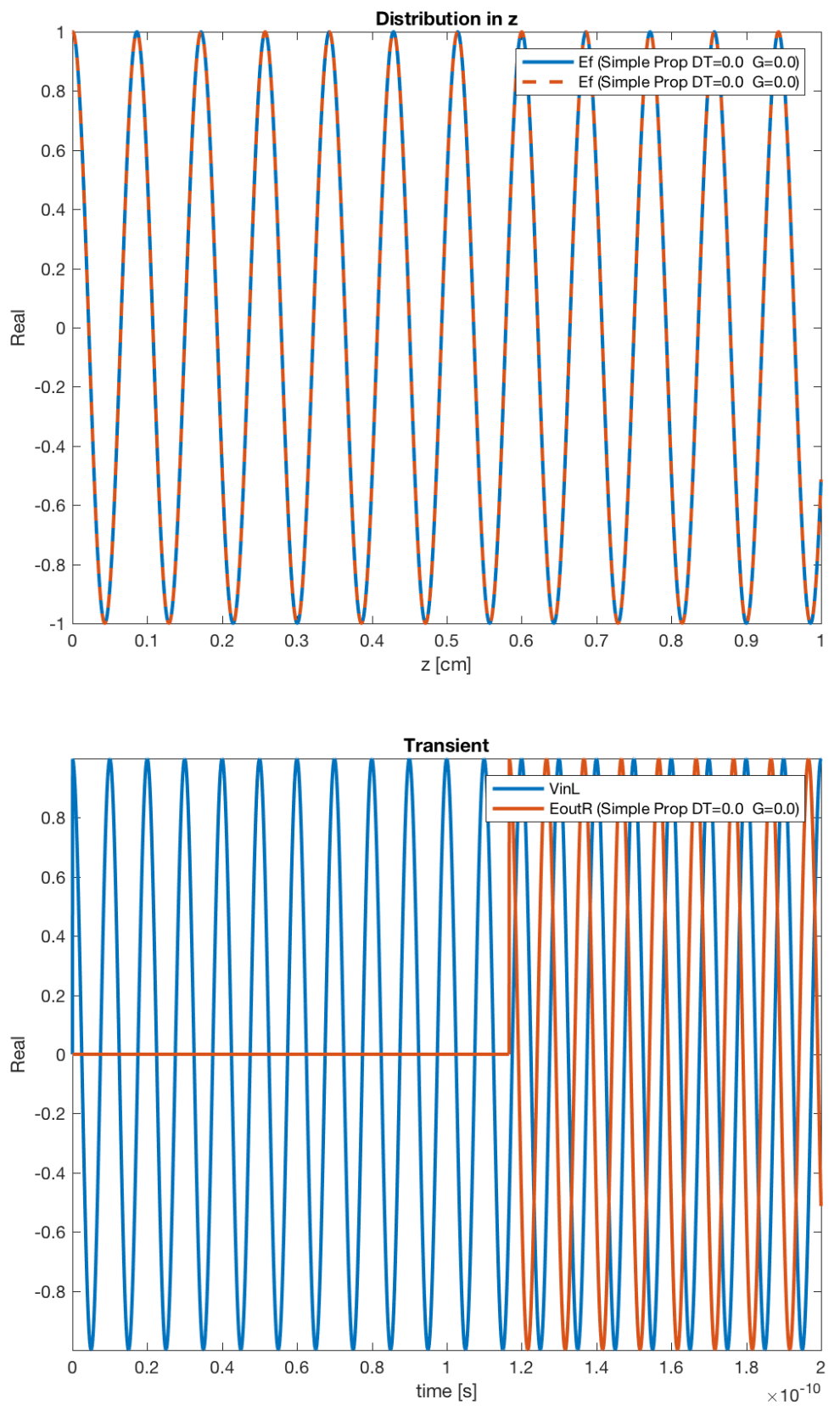

Figure 5.1: Traveling Wave propagation with zero $\beta$ : no gain and output at same frequency with delay. 


\subsection{Real $\beta$}

Let's explore solutions to the traveling wave equation with non-zero terms on the right-hand side. Starting with $\beta$ real we have:

$$
\left(\frac{\partial}{\partial t}+v \frac{\partial}{\partial z}\right) E(z, t)=-i v \beta E(z, t)
$$

As before a solution to this will be a plane wave. We chose $k_{\beta}$ not to presume the same requirement as in the zero $\beta$ case.

$$
E(z, t)=A_{0} e^{i\left(k_{\beta} z-\omega_{e} t\right)}
$$

Plugging this into the PDE we get

$$
\begin{aligned}
-i \omega_{e} E(z, t)+i v k_{\beta} E(z, t) & =-i v \beta E(z, t) \\
-\omega_{e}+v k_{\beta} & =-v \beta \\
k_{\beta} & =\frac{\omega_{e}}{v}-\beta
\end{aligned}
$$

where again $v$ is the phase velocity. If we use (5.5) this is also

$$
k_{\beta}=k_{e}-\beta
$$

We see that the wave vector or propagation constant has been modified by $\beta$ which will have the same units as $k_{e}$. The group velocity (envelope phase velocity) will also change accordingly:

$$
v_{\beta}=\frac{\omega_{e}}{k_{\beta}}
$$

We see that a real $\beta$ has the effect of changing the spatial frequency as the wave propagates through the medium. In a 3-D plot over $z$ the wave will rotate. This can be seen in figure B.1. In Figure 5.2 we see that the time domain picture has the same frequency but the $z$-domain picture has a different spatial frequency. This is equivalent to a change of index $n$, or, since $n$ is not directly used in the model, we can imagine that $n$ has remained the same and the length of the active layer has changed. This is known as detuning. In resonant cavities (see section 5.9) this change in $n$ causes a different optical length and so different $\lambda$ 's for the modes.

If we start with the plane wave solution

$$
E(z, t)=A_{0} e^{i\left(k_{\beta} z-\omega_{e} t\right)}
$$

and set the input to be a constant so that

$$
E(0, t)=A_{0}
$$

this requires that

$$
E(z, t)=A_{0} e^{i k_{\beta} z}
$$


Note that the input is a constant even though the solution to the PDE is not. To verify this is a solution to the PDE we plug it in and get

$$
\begin{aligned}
i v k_{\beta} E(z, t) & =-i v \beta E(z, t) \\
k_{\beta} & =-\beta
\end{aligned}
$$

So we see that a real $\beta$ will offset the spatial frequency while leaving the wave frequency unchanged. This can be viewed as adding a rotation over $z$ to the signal phase. If the input is constant it will now rotate and end with a new phase. If the input is sinusoidal it will rotate faster or slower depending on the sign of $\beta$ and again come out with a different phase. The frequency in time and the amplitude will not be affected.

If we use a sinusoid input instead of complex exponential things are not as easy to see. In figure 5.3 it is difficult to see the spatial frequency changes because it is the sum of two complex exponentials. We can show (see appendix B) that the two waves rotating in opposite directions have different spatial frequencies and speeds, and when you add them the speed of the sum is not changed and the result is that shown in the figure. The figure can be understood better if you realize that the phase is changing; i.e. the sinusoid is being twisted and is why it appears to shrink in amplitude. The imaginary part is growing at these points. See figure B.3 in the appendix. 



Figure 5.2: Traveling wave propagation with real $\beta$ : no gain and no frequency shift, but a spatial frequency shift. 

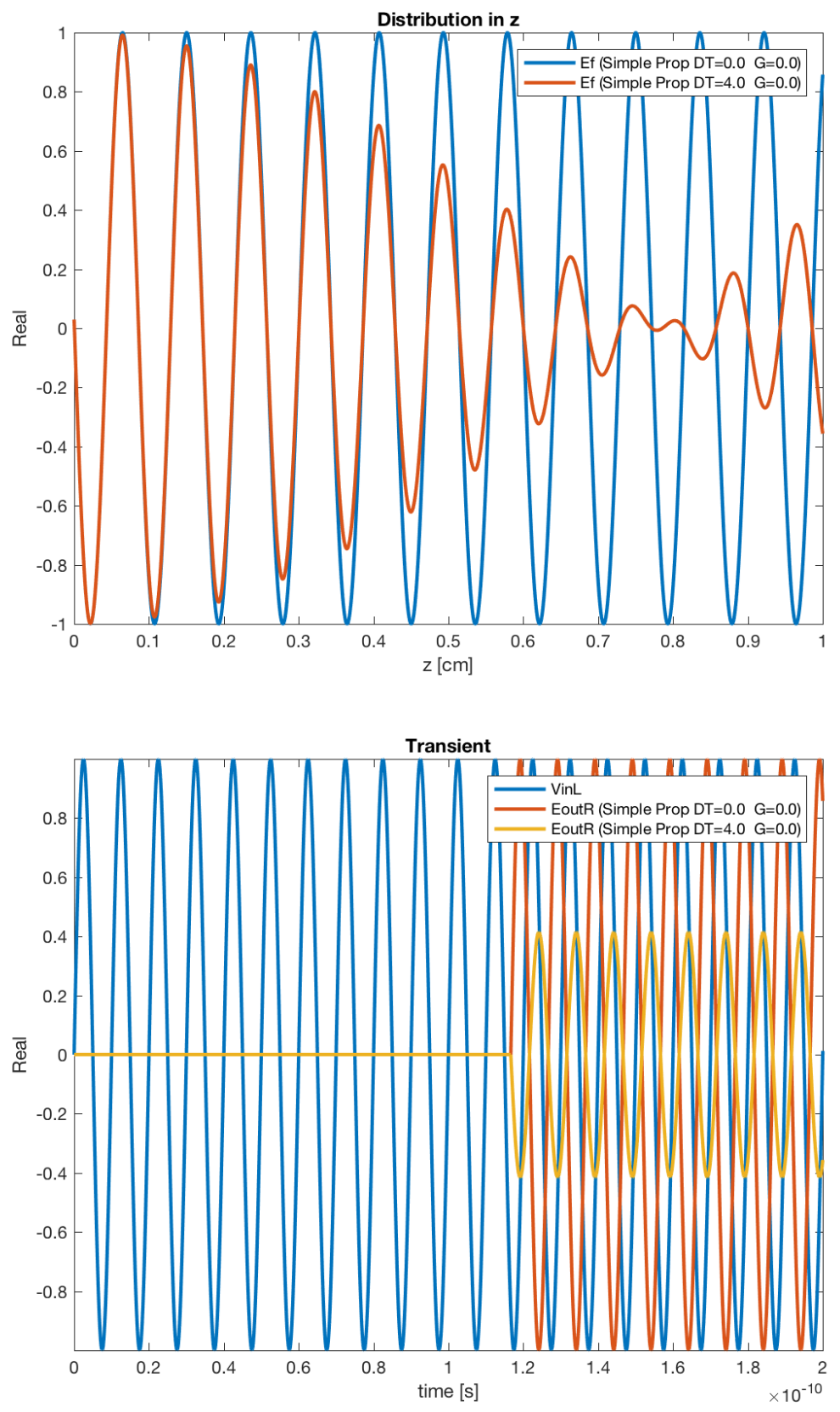

Figure 5.3: Traveling Wave propagation with real $\beta$ using sinusoidal input: no gain and no frequency shift. Show in 3D? 


\subsection{Imaginary $\beta$}

Let's now explore solutions to the traveling wave equation with an imaginary $\beta$ term on the right-hand side.

$$
\left(\frac{\partial}{\partial t}+v \frac{\partial}{\partial z}\right) E(z, t)=-i v \beta E(z, t)=v \alpha E(z, t)
$$

where we have let $\beta=i \alpha$. The solution is a plane wave as before. Use $k_{\alpha}$ not to presume the same requirement as in the previous cases.

$$
E(z, t)=A_{0} e^{i\left(k_{\alpha} z-\omega_{e} t\right)}
$$

Plugging this into the PDE we get

$$
\begin{aligned}
-i \omega_{e} E(z, t)+i v k_{\alpha} E(z, t) & =v \alpha E(z, t) \\
-\omega_{e}+v k_{\alpha} & =-i v \alpha \\
k_{\alpha} & =\frac{\omega_{e}}{v}-i \alpha
\end{aligned}
$$

where again $v$ is the phase velocity. If we use (5.5) this is also

$$
k_{\alpha}=k_{e}-i \alpha
$$

Comparing this with (5.11) it is the same (as it must be) if we replace $i \alpha$ with $\beta$. This result is more enlightening if we put this back into (5.16)

$$
\begin{aligned}
E(z, t) & =A_{0} e^{i\left(k_{e} z-i \alpha z-\omega_{e} t\right)} \\
& =A_{0} e^{\alpha z} e^{i\left(k_{e} z-\omega_{e} t\right)}
\end{aligned}
$$

This shows that the solution is a wave that stays the same except for the magnitude. See figure 5.4. This is the same for the propagation constant where the imaginary part controls the gain or attenuation.

Unlike before if we use a sinusoid input instead of a complex exponential there is no change. This is because there is no rotation. See Figure 5.5. 

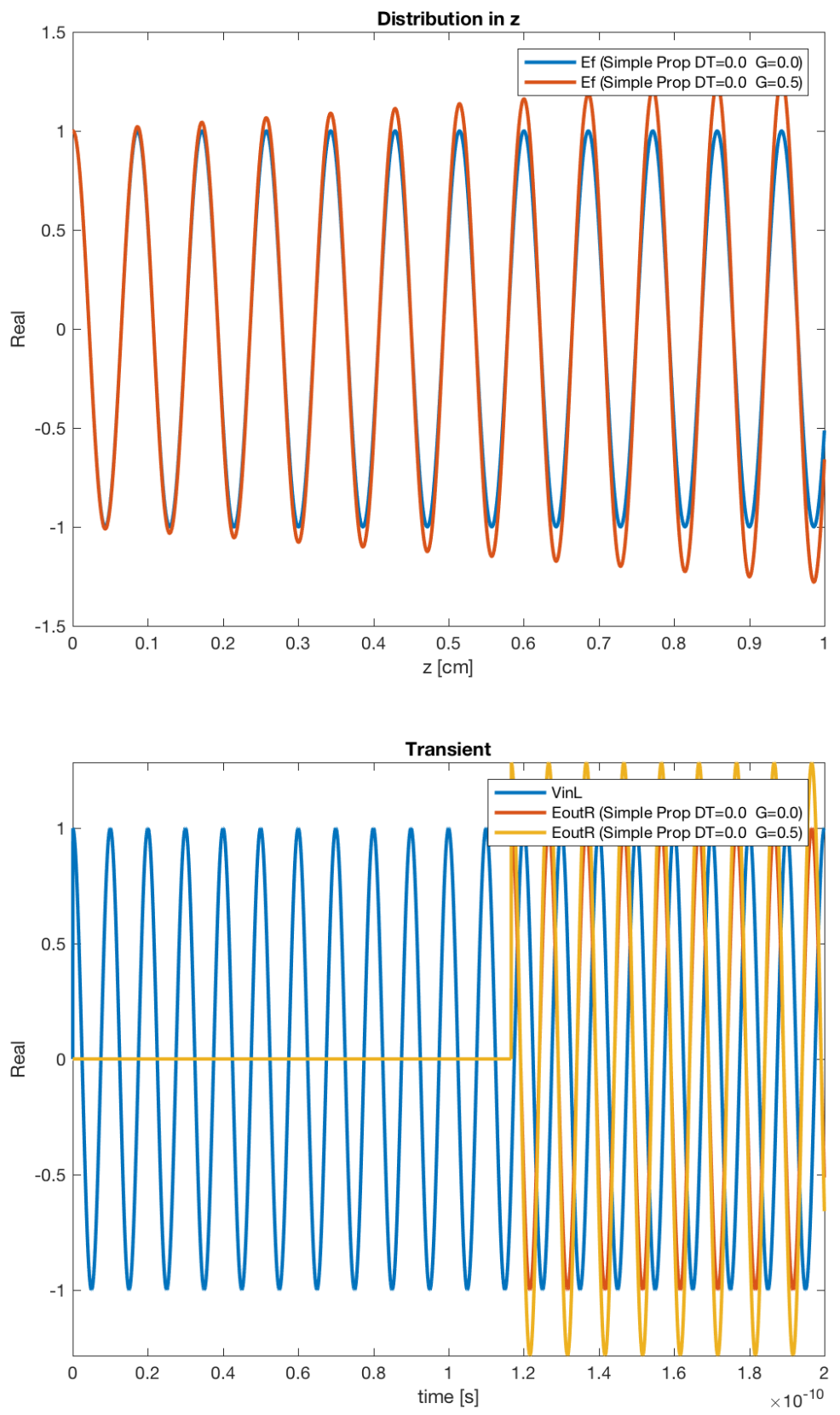

Figure 5.4: Traveling Wave propagation with imaginary $\beta$ : Gain with no frequency shift. 

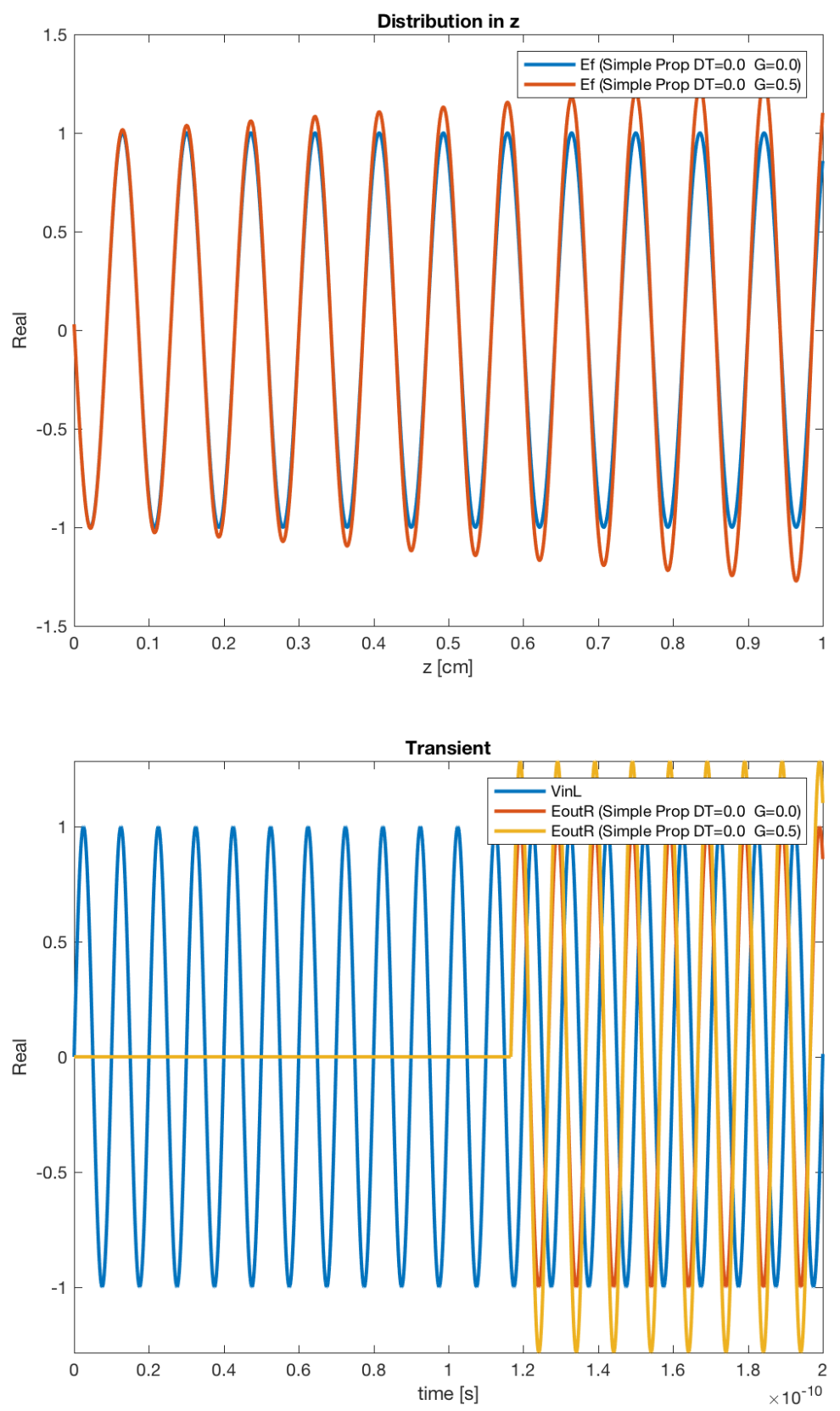

Figure 5.5: Traveling Wave propagation with imaginary $\beta$ using sinusoidal input: Gain with no frequency shift. 


\subsection{Finite-Difference Time-Domain Method}

Up to this point we have been studying the TWM equations analytically to get a better understanding on what they mean. Since our model will need to employ numerical methods we need to review the method that we will be using.

The Finite-Difference Time-Domain method or (FDTD) is one of many numerical methods. It is based on the Finite-Difference Method (FDM) takes a system of DEs (or integral equations) and transforms it into a system of linear algebraic equations which are easily handled by digital computers. A domain within the problem is broken into small regions or discretized. FDTD is a specific type of FDM where the time domain is also discretized. We will give a brief overview with the intention of using these techniques to explore the laser models of this thesis. This information is covered in many texts including [31].

The main reason for numerical modeling in general is that many problems are not solvable analytically. And even if they are, numerical modeling can handle much more complex geometries and material properties than would otherwise be possible. Accuracy and efficiency are important factors in evaluating numerical methods. We will find that the MNA engine has become applicable to many more problems as computer power and memory increase making the implicit equation solvers tenable to them.

\subsubsection{Algebraic Difference Equations}

Although FDM applies to integral equations as well we will only cover the finite-difference approximation to a differential equation. The Taylor series of a function is used to give approximations of a desired accuracy for differentials of any order. The Taylor series of a function $f(x)$ about a point $a$ is

$$
\begin{aligned}
f(x) & =\sum_{k=0}^{\infty} \frac{f^{(k)}(a)(x-a)^{k}}{k !} \\
& =f(a)+f^{\prime}(a)(x-a)+\frac{f^{\prime \prime}(a)(x-a)^{2}}{2 !}+\frac{f^{\prime \prime \prime}(a)(x-a)^{3}}{3 !}+\ldots
\end{aligned}
$$

For simplicity we discretize the $x$ domain into equal intervals $x_{i+1}-x_{i}=h$. Writing the Taylor series at these discrete points about the point $a=x_{i}$ we get

$$
f\left(x_{i+1}\right)=f\left(x_{i}\right)+f^{\prime}\left(x_{i}\right) h+\frac{f^{\prime \prime}\left(x_{i}\right) h^{2}}{2 !}+\frac{f^{\prime \prime \prime}\left(x_{i}\right) h^{3}}{3 !}+O\left(h^{4}\right)
$$

where $O\left(h^{4}\right)$ means of order. This gives us an upper bound on the error of our approximation. 
Similarly we can get estimates for all of our domain points:

$$
\begin{aligned}
& f\left(x_{i-1}\right)=f\left(x_{i}\right)-f^{\prime}\left(x_{i}\right) h+\frac{f^{\prime \prime}\left(x_{i}\right) h^{2}}{2 !}-\frac{f^{\prime \prime \prime}\left(x_{i}\right) h^{3}}{3 !}+O\left(h^{4}\right) \\
& f\left(x_{i+2}\right)=f\left(x_{i}\right)+f^{\prime}\left(x_{i}\right)(2 h)+\frac{f^{\prime \prime}\left(x_{i}\right)(2 h)^{2}}{2 !}+\frac{f^{\prime \prime \prime}\left(x_{i}\right)(2 h)^{3}}{3 !}+O\left(h^{4}\right) \\
& f\left(x_{i-2}\right)=f\left(x_{i}\right)-f^{\prime}\left(x_{i}\right)(2 h)+\frac{f^{\prime \prime}\left(x_{i}\right)(2 h)^{2}}{2 !}-\frac{f^{\prime \prime \prime}\left(x_{i}\right)(2 h)^{3}}{3 !}+O\left(h^{4}\right) \\
& f\left(x_{i+3}\right)=f\left(x_{i}\right)+f^{\prime}\left(x_{i}\right)(3 h)+\frac{f^{\prime \prime}\left(x_{i}\right)(3 h)^{2}}{2 !}+\frac{f^{\prime \prime \prime}\left(x_{i}\right)(3 h)^{3}}{3 !}+O\left(h^{4}\right) \\
& f\left(x_{i-3}\right)=f\left(x_{i}\right)-f^{\prime}\left(x_{i}\right)(3 h)+\frac{f^{\prime \prime}\left(x_{i}\right)(3 h)^{2}}{2 !}-\frac{f^{\prime \prime \prime}\left(x_{i}\right)(3 h)^{3}}{3 !}+O\left(h^{4}\right)
\end{aligned}
$$

Finite-difference approximations for differentials are then constructed from linear combinations of these Taylor expansions with the order being easily determined by the error terms.

Let's look at the first-order in $h$ approximations to $f^{\prime}(x)$. From (5.22)

$$
f\left(x_{i+1}\right)=f\left(x_{i}\right)+f^{\prime}\left(x_{i}\right) h+O\left(h^{2}\right)
$$

and solving for $f^{\prime}\left(x_{i}\right)$ we get

$$
f^{\prime}\left(x_{i}\right)=\frac{f\left(x_{i+1}\right)-f\left(x_{i}\right)}{h}+O(h)
$$

This is called a forward difference (or forward Euler after the discoverer ). A plot of the function and its approximation is shown in figure 5.6. Note how the derivative at $x$ is based on the value at $x_{i}$ and a value forward of it.

Then from (5.23)

$$
f\left(x_{i-1}\right)=f\left(x_{i}\right)-f^{\prime}\left(x_{i}\right) h+O\left(h^{2}\right)
$$

and

$$
f^{\prime}\left(x_{i}\right)=\frac{f\left(x_{i}\right)-f\left(x_{i-1}\right)}{h}+O(h)
$$

This is called a backward difference (or backward Euler) and is shown in figure 5.7 and here the derivative at $x$ is based on the value at $x$ and a value back from it.

We can continue with the second-order in $h$ approximations. If we subtract (5.23) from (5.22) we get

$$
f^{\prime}\left(x_{i}\right)=\frac{f\left(x_{i+1}\right)-f\left(x_{i-1}\right)}{2 h}+O\left(h^{2}\right)
$$




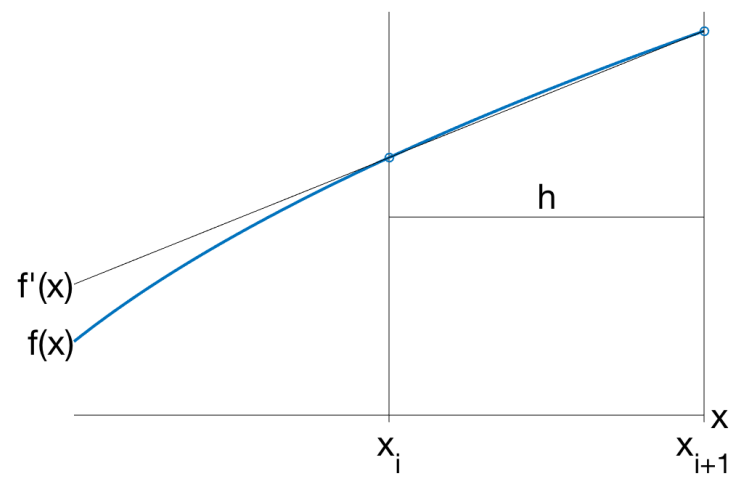

Figure 5.6: Plot of $f(x)$ showing the forward difference - the derivative at $x_{i}$ calculated from the value at the same $x_{i}$ and the value forward of it $x_{i+1}$

This is a central difference equation. Note the increased accuracy with still only two points. Or if we add (5.23) and (5.22) we get a second-order central difference to $f^{\prime \prime}\left(x_{i}\right)$

$$
f^{\prime \prime}\left(x_{i}\right)=\frac{f\left(x_{i+1}\right)-2 f\left(x_{i}\right)+f\left(x_{i-1}\right)}{h^{2}}+O\left(h^{2}\right)
$$

So far we have been discretizing space in the $x$ dimension. There is nothing special about this dimension and we could discretize any of the spatial dimensions or time if we chose. This is exactly what we do for the FDTD.

\subsubsection{Solving a Differential Equation}

Once we have chosen a finite-difference approximation using it is a simple matter of substituting it in for the differentials in the DE. Let's take the example of the DE of a low-pass $R C$ filter.

$$
\frac{d V_{o}}{d t}=\frac{1}{R C}\left(V_{i}-V_{o}\right)
$$

Let's first solve it using the forward Euler approximation (5.28)

$$
\frac{d V_{o}}{d t} \simeq \frac{V_{o}^{n+1}-V_{o}^{n}}{h}
$$

where we have replaced $f\left(t_{i}\right)$ with $V_{o}^{n}$. Substituting this into (5.32) we get

$$
\begin{aligned}
\frac{V_{o}^{n+1}-V_{o}^{n}}{h} & =\frac{1}{R C}\left(V_{i}^{n}-V_{o}^{n}\right) \\
V_{o}^{n+1} & =\left(1-\frac{h}{R C}\right) V_{o}^{n}+\frac{h}{R C} V_{i}^{n}
\end{aligned}
$$




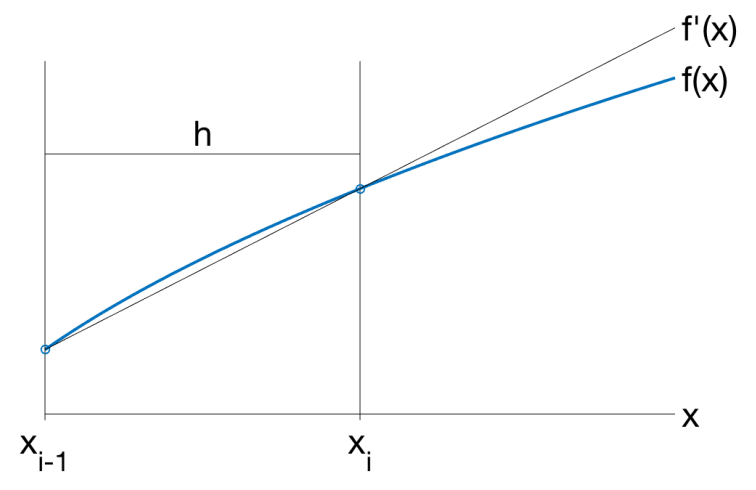

Figure 5.7: Plot of $f(x)$ showing the backward difference - the derivative at $x_{i}$ calculated from the value at the same $x_{i}$ and the value backward from it $x_{i-1}$.

Then, given (or assuming zero) initial conditions for $V_{o}^{n}$ we can calculate the next value $\left(V_{o}^{n+1}\right)$ based on the present value $\left(V_{o}^{n}\right)$ and present value input $\left(V_{i}^{n}\right)$. When the equation output is based only on previous values we call this an explicit difference equation. It is very simple to solve as we just start at an initial condition and march forward through time.

Let's now solve our DE using the backward Euler approximation (5.29)

$$
\frac{d V_{o}}{d t} \simeq \frac{V_{o}^{n}-V_{o}^{n-1}}{h}
$$

where we have again replaced $f\left(t_{i}\right)$ with $V_{o}^{n}$. Substituting this into (5.32) we get

$$
\begin{aligned}
\frac{V_{o}^{n}-V_{o}^{n-1}}{h} & =\frac{1}{R C}\left(V_{i}^{n}-V_{o}^{n}\right) \\
V_{o}^{n}-V_{o}^{n-1} & =\frac{h}{R C}\left(V_{i}^{n}-V_{o}^{n}\right) \\
\left(1+\frac{h}{R C}\right) V_{o}^{n} & =V_{o}^{n-1}+\frac{h}{R C} V_{i}^{n} \\
V_{o}^{n} & =\left(\frac{R C}{R C+h}\right)\left\{V_{o}^{n-1}+\frac{h}{R C} V_{i}^{n}\right\}
\end{aligned}
$$

Notice how the present value of the derivative is based on the present and previous values of the system variables. This makes it an implicit equation and will be more difficult to solve. An important property of implicit equations is that they are stable. As mentioned before a full nodal analysis using the MNA solver is implicit. It requires inverting large matrices as apposed to simple marching through time like the explicit equation. The stability factor makes it worthwhile. 


\subsubsection{Finite-Difference in Time and Space}

We have seen that the FDM method can be discretized in a space dimension or a time dimension. FDTD is simply what we call an FDM that has been discretized in time and space. The same substitutions are made but now the differential equations have two substitutions for each of the time and space derivatives. The calculations must be done sequentially, first evaluating the space portion and then advancing time and repeating.

\subsubsection{Upwind Finite Difference Scheme}

The upwind scheme discretizes the time domain and the $z$-domain but does so taking into account the direction of travel of the field. For the 1-D forward traveling wave equation

$$
\frac{\partial E}{\partial t}+v \frac{\partial E}{\partial z}=0
$$

we have two choices for the derivative substitutions. Upwind

$$
\frac{\partial E}{\partial t} \simeq \frac{E_{k}^{n}-E_{k}^{n-1}}{\triangle t}, \quad \frac{\partial E}{\partial z} \simeq \frac{E_{k}^{n-1}-E_{k-1}^{n-1}}{\triangle z}
$$

or downwind

$$
\frac{\partial E}{\partial t} \simeq \frac{E_{k}^{n}-E_{k}^{n-1}}{\triangle t}, \quad \frac{\partial E}{\partial z} \simeq \frac{E_{k+1}^{n-1}-E_{k}^{n-1}}{\triangle z}
$$

Both of these work because the first-order approximation cannot be centered about $k$.

Using (5.38) in the simplified traveling wave equation (5.37), we get

$$
\begin{aligned}
\frac{E_{k}^{n}-E_{k}^{n-1}}{\triangle t}+v \frac{E_{k}^{n-1}-E_{k-1}^{n-1}}{\triangle z} & =0 \\
E_{k}^{n} & =E_{k}^{n-1}+\frac{v \triangle t}{\triangle z}\left(E_{k-1}^{n-1}-E_{k}^{n-1}\right)
\end{aligned}
$$

If we set $\frac{v \triangle t}{\Delta z}=1$ this reduces to

$$
E_{k}^{n}=E_{k-1}^{n-1}
$$

Because the DE in question is the traveling wave equation we realize that the time stepping is marching in time by simply translating each point in the initial solution to the new position $x+v \triangle z$. Thus if we watch the $E_{k}^{n}, E_{k-1}^{n}$ points they will be shifted to the right. If we think of the wave moving downwind then these points were upwind and is where the scheme gets its name. This is a natural choice as these points are causally connected with $E_{k}^{n+1}$. But this physical argument is not the real reason we use the upwind scheme. 
Consider the other possible choice (5.39) for the derivative approximations.

$$
\begin{aligned}
\frac{E_{k}^{n}-E_{k}^{n-1}}{\triangle t}+v \frac{E_{k+1}^{n-1}-E_{k}^{n-1}}{\triangle z} & =0 \\
E_{k}^{n} & =E_{k}^{n-1}+\frac{v \triangle t}{\triangle z}\left(E_{k}^{n-1}-E_{k+1}^{n-1}\right) \\
E_{k}^{n} & =2 E_{k}^{n-1}-E_{k+1}^{n-1}
\end{aligned}
$$

This is approximately (to first-order) equivalent to (5.39) but will not be as exact as the upwind where the two terms cancel.

Because the upwind scheme takes into account the direction of travel it will be different for the wave traveling in the reverse direction. This complicates the implementation because the forward and reverse traveling waves will have different derivative approximations. It does, however, produce a model with no numerical dispersion. This of course is only strictly true when the right-hand side of the first-order wave equations are 0 . The upwind scheme is stable if [32]

$$
\left|\frac{v \triangle t}{\triangle z}\right| \leq 1
$$

which is known as the Courant number. This is excellent as it works with our synchronization condition.

So even though the wave equations are only first-order, if the upwind scheme is used we have no dispersion and a guaranteed stable system. This is a very important factor in choosing the first-order wave equations for numerical analysis.

\subsection{Discretization}

The discretization of the first-order traveling wave equations is then straightforward. Using a first-order linear approximation we make the upwind substitutions from section 5.5.4

$$
\frac{\partial E}{\partial t} \simeq \frac{E_{k}^{n}-E_{k}^{n-1}}{\triangle t}, \quad \frac{\partial E}{\partial z} \simeq \frac{E_{k}^{n-1}-E_{k-1}^{n-1}}{\triangle z}
$$

for the time and $z$ derivatives. Starting with the simplified traveling wave equations (5.3) (only showing the forward traveling wave equation), we get

$$
\begin{aligned}
\frac{E_{k}^{n}-E_{k}^{n-1}}{\triangle t}+v \frac{E_{k}^{n-1}-E_{k-1}^{n-1}}{\triangle z} & =0 \\
E_{k}^{n} & =E_{k}^{n-1}+\frac{v \triangle t}{\triangle z}\left(E_{k-1}^{n-1}-E_{k}^{n-1}\right)
\end{aligned}
$$

If we set $\frac{v \triangle t}{\triangle z}=1$ this reduces to

$$
E_{k}^{n}=E_{k-1}^{n-1}
$$


As is detailed in section 5.5.4 there are two choices which are usually equally valid - one may be chosen over the other because of boundary conditions. In both cases of the wave traveling forward and reverse it is very important that we choose the 'upwind' evolution scheme. Again, they will be different for each direction and reduces the numerical dispersion of the model drastically. It also guarantees a stable solution to the first-order wave equations. The caveat is that the forward and reverse waves cannot be treated the same.

Setting $\frac{v \Delta t}{\Delta z}=1$ may also seem like a sensible simplification to either save computing time or just to make the implementation cleaner. This is not the case. By making this condition true and using the 'upwind' evolution we actually reduce our error to zero for simple propagation. Remember that up to this point we have discretized the simplified traveling wave equations which have the right-hand side set to 0 .

As can be seen in (5.43) the $z$ values will just be shifted from one slice to the next - the values are copied exactly as they are introducing no additional error! This is critical to the success of this approximation. It makes a first-order implementation not only simple but accurate as well. The huge drawback to this simplification which we call the synchronization condition is that the time step of our simulation is fixed $\left(\Delta t=\frac{\Delta z}{v}\right)$. This means that even though the user may have reasons for choosing his own time step this model will still have to use its own.

For the more general situation where the right-hand side of the traveling wave equation is non-zero we start with (5.43) and add the $\beta$ term to get

$$
\begin{gathered}
E_{k}^{n}=E_{k-1}^{n-1}-i \triangle z \beta E_{k-1}^{n-1} \\
E_{k}^{n}=(1-i \triangle z \beta) E_{k-1}^{n-1}
\end{gathered}
$$

We replace this with

$$
E_{k}^{n}=E_{k-1}^{n-1} e^{-i \beta \triangle z}
$$

A simple way to understand this is by observing that the change in phase per $\triangle z$ is

$$
\frac{E_{k}^{n}}{E_{k-1}^{n-1}}=1-i \triangle z \beta
$$

and

$$
\triangle \phi=-\tan ^{-1}(\triangle z \beta)
$$

which by inspection is equal to the phase change of (5.45). The reason this substitution is incorporated is for better performance. Even though at small $\triangle z$ the approximation is excellent, over the course of the simulation there will be a small cumulative error. By recognizing the exact function being approximated we will not only be more accurate, but we will be more accurate for larger slices as well. This allows us to have reasonably accurate simulations with much fewer slices which will greatly increase the simulation speed.

Another way to look at this substitution is on energy conservation grounds. If we don't make the substitution the model will slowly lose energy. 


\subsection{Traveling Wave Model with Dispersion}

For a semiconductor model we need to capture frequency dependencies of the lasing medium. The dispersion relation - where different wavelengths travel at different speeds - is discussed in section 2.5. The linewidth of most mediums can be modeled fairly well by a Lorentzian (refer to section 2.3.3). This is also used in the multi-mode REM to give the mode suppression as shown in section 3.6. For semiconductor lasers the Lorentzian is not the best model as it is not symetrical and exhibits a 'tail'. A good approximation can be achieved by a sum of Lorentzians [33]. For our model we can use any linear function that can be coded so the single Lorentzian is a reasonable distribution to work with.

In the TWM this is done by adding a term on the right-hand side of the traveling wave equation. The term is derived from Maxwell's equations following [26]. It assumes $\varepsilon(\omega)$ is a linear function of $\omega$ in area of interest and then a Lorentzian is fit to take into account the actual gain dispersion. Although this adds complexity it doesn't increase the computational effort. [26] The result (for the forward traveling wave) is

$$
\left(\frac{\partial}{\partial t}+v \frac{\partial}{\partial z}\right) E=-i v \beta E-g_{p}(E-p)
$$

where $p$ is the polarization defined in the frequency domain as

$$
p(\omega)=E(\omega) \frac{\gamma_{p}}{\gamma_{p}+i\left(\omega-\omega_{0}\right)}
$$

and in the time domain by

$$
\frac{d p}{d t}=i \omega_{0} p+\gamma_{p}(E-p)
$$

where $\gamma_{p}$ is the Lorentzian width and $\omega_{0}$ is the center frequency. Remember that a center frequency of 0 will correspond to the implied carrier frequency. If possible $\omega_{0}$ is chosen to be the fundamental mode of the resonant cavity $\left(\omega_{0}=0\right)$, but this is not always possible. This eliminates the need to keep track of the carrier phase in system simulations as explained in section A.2. Remember that $\omega_{0}$ defines the center of the Lorentzian relative to the carrier $\omega_{c}$. In our FDTD simulations we use the polarization DE (5.48).

Figures 5.8 and 5.9 show the typical spreading and attenuation of a pulse from a Lorentzian dispersion relationship. Note that because of the symmetry of the complex Lorentzian term any real signal will pass through and remain real as the complex parts cancel each other.

The speed of the traveling waves are determined by the group velocity which is a constant for the TWM. This does not mean that all waves will travel at the same speed. As explained in section 5.3 when a detuning factor is specified on the right-hand side of the traveling wave equation we effectively change the index and speed. Since the Lorentzian adds a real and imaginary term to the right-hand side it will affect the speed. The gain (or imaginary part of the index) is obviously affected by the polarization Lorentzian. The change to the real part of the index is small compared to the imaginary and so the dominant effect will be the gain over frequency. 

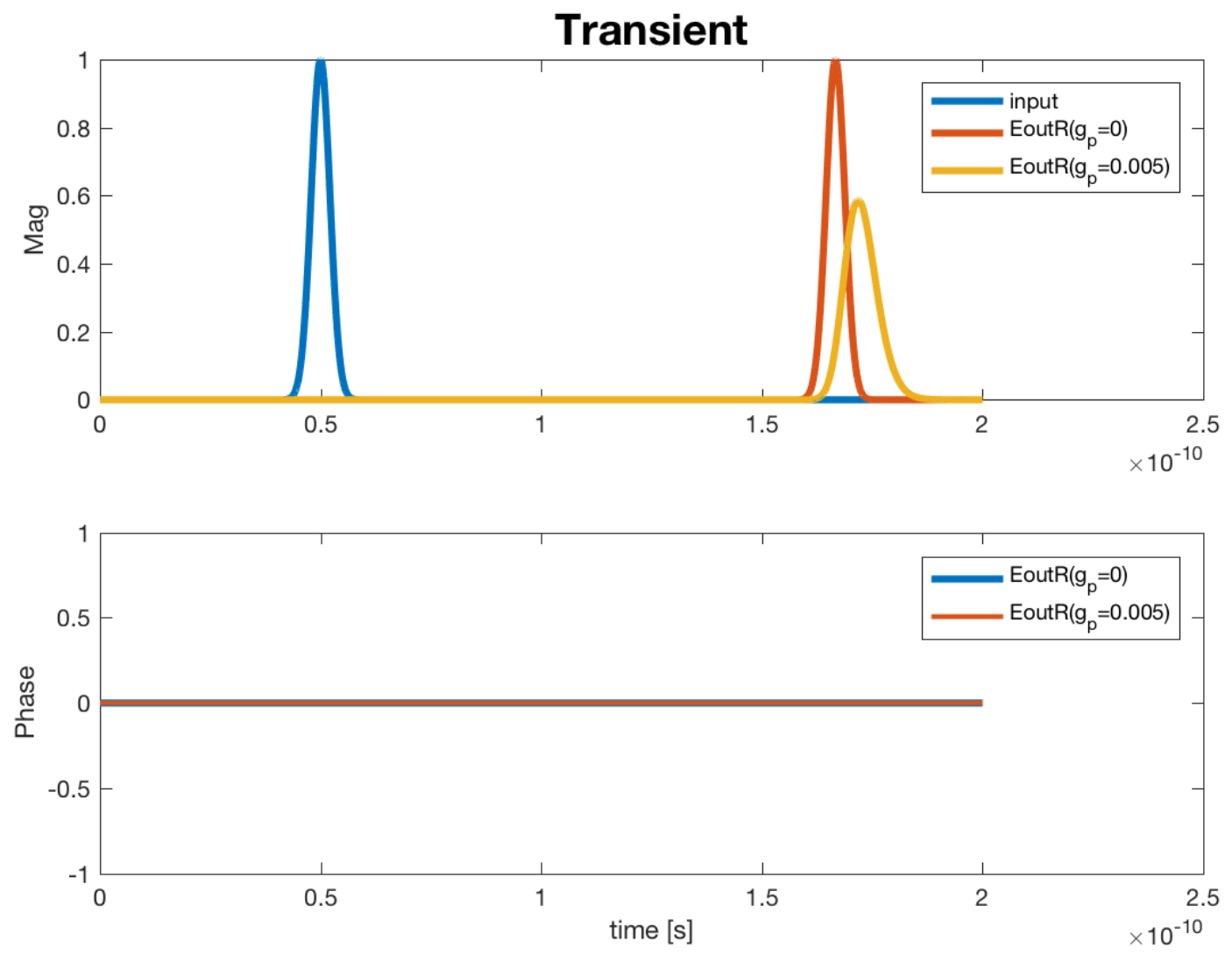

Figure 5.8: TWM Gaussian pulse propagation. $\gamma_{p}=2 \pi\left(2 \times 10^{11}\right)[\mathrm{rad} / \mathrm{s}]$. Note that if we observed the pulse propagate through the waveguide we would see it shrinking and widening. 

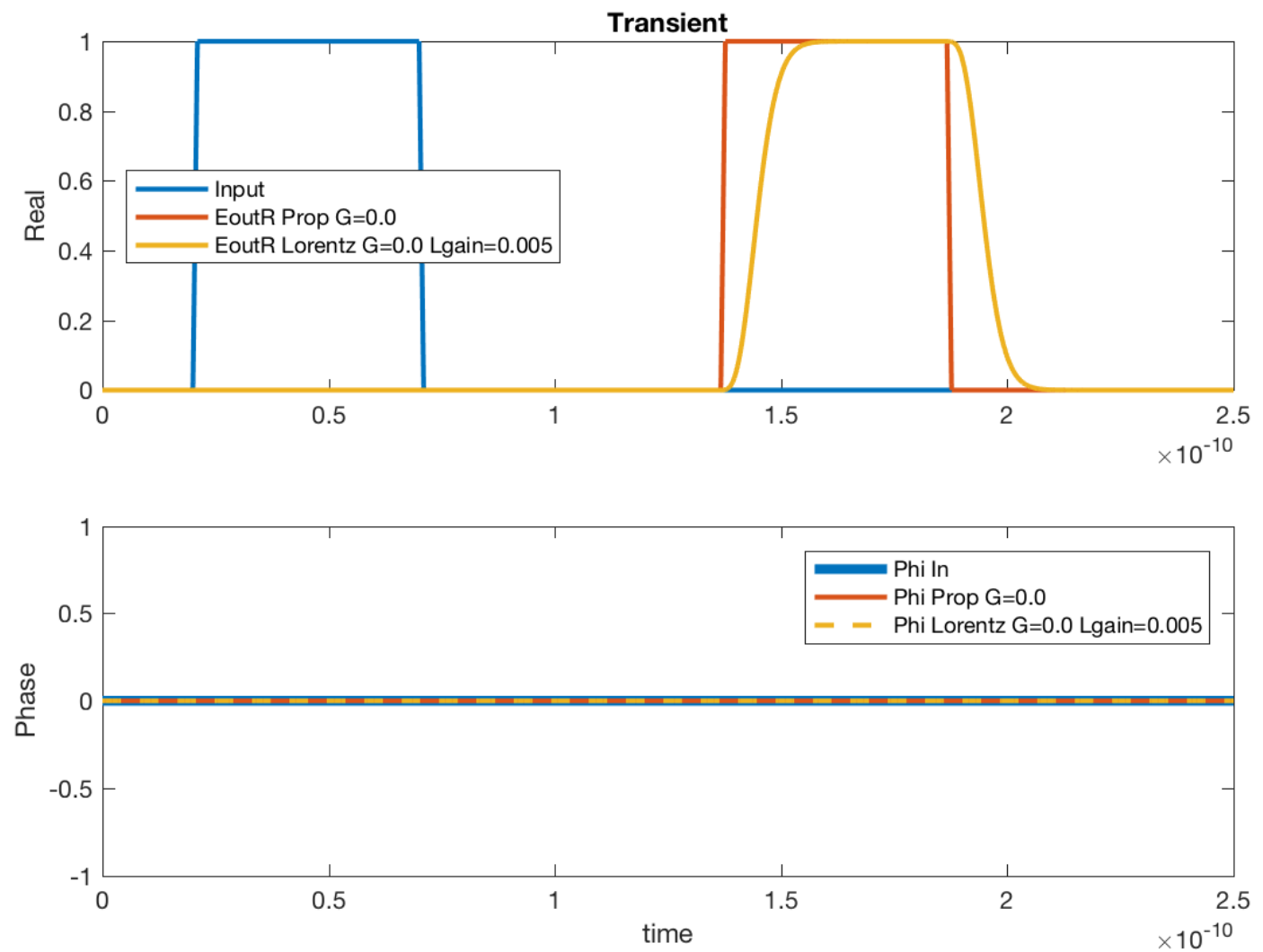

Figure 5.9: TWM rectangular pulse propagation. Note the phase is always 0 only because $\omega_{0}=0$ which means the Lorentzian has no imaginary term. 


\subsection{Traveling Wave Model with Advanced Terms}

There are many features of optical media that we may wish to model. The most common ones being coupling between the forward and reverse traveling waves and spontaneous emission. Coupling is modeled by a coupling factor $\kappa$ and could be used in modeling a DFB laser, for example. Spontaneous emission can be modeled by an extra term $F_{s p}$ representing random noise. This is essential in laser modeling and in particular to the TWM. Because of the physical nature of the TWM it relies on spontaneous emission to generate photons and stimulated emission.

$$
\left(\frac{1}{v} \frac{\partial}{\partial t} \pm \frac{\partial}{\partial z}\right) E_{ \pm}(z, t)=-i \beta E_{ \pm}(z, t)-i \kappa_{\mp} E_{\mp}(z, t)+F_{s p}(z, t)
$$

The literature has many examples of adding properties to the TWM. They tend to be specific to some problem and are not of interest in this research other than to say the TWM lends itself to adding specialized features (including nonlinear affects) by additional terms.

\subsection{Traveling Wave Model Resonant Cavity and De- tuning}

Since we want to use the TWM for a laser model we begin with how it would work with a resonant cavity. Up to now we have treated the TWM as an optical amplifier with an input on the left and the output on the right and have also ignored the reverse traveling wave. This will not work for a resonant cavity. Both waves need to be propagated and mirrors added on both ends. Although the model can implement phase shifts at reflection points as desired - as well as any reflection and transmission coefficients - for now we stick with perfect reflectors at each end with no phase shift.

Also to keep things simple to better understand what is going on with the model we will run the TWM without the $N$ equation. This means that if the model were 'turned on' with no photons in the cavity nothing would happen because we are not injecting current and and calculating carriers. Hence they could not build up and eventually recombine and release photons. In our example we will have no optical input because we wish to observe the laser properties of the cavity. We could use the $N$ equation, a current source with spontaneous emission as required in a real laser. This would lead to stimulated emission and build up the modes but would take a long time. It is easy to preload the model cavity with any distribution of photons we like and so we initialize it with a wave which of course represents a certain photon density and distribution over $z$. In this case we will preload the cavity with one of the allowed modes. One would expect that if this is not an allowed mode it would be eliminated but again to isolate the cavity effects we will also not turn on the Lorentzian filter. The proof that this is a valid cavity mode is that the sinusoid we introduce has no discontinuities which would show up as significant energy in higher modes.

The first simulation has no detuning and also no Henry factor leaving the right-hand side of the TWM equations zero. The energy is found at the preloaded wave frequency verifying 
that we did actually preload the cavity with a valid mode. Then detuning is added via the static detuning factor $\delta$ in (5.15). It is one of the real $\beta$ parameters and as described above (section 5.3) a real $\beta$ will model a change in the index which would then shift the modes of the cavity. As $\delta$ is increased the mode shift increases with it. Figure 5.10 shows the TWM with several values of static detuning $\delta$. As we increase $\delta$ from 2 to 10

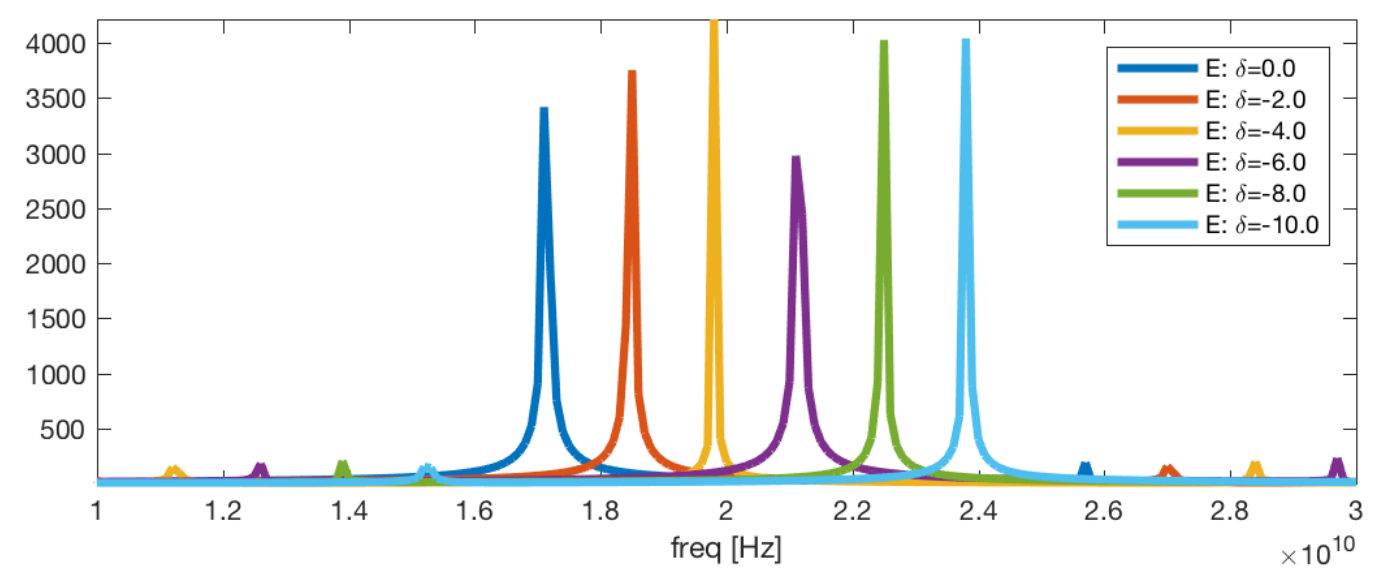

Figure 5.10: TWM resonant cavity modes determine $k$ and hence $\lambda$

It is worthwhile to emphasize that because the carrier is implicit these mode shifts represent shifts of the carrier frequency, or detuning of the carrier. This is necessary if two TWM do not have the same carrier and we wish to use them in the same system simulation. Refer to appendix A.5.

Another use of detuning is keeping track of the carrier phase when two signals with different carriers propagate through TWM. Normally we would choose the carrier to be a mode of the cavity and eliminate this need but it cannot always be done. An example of how this is done is given in appendix A.2, A.3, and A.4. 


\section{Chapter 6}

\section{Traveling Wave Laser Model}

The Traveling Wave Laser Model is just the TWM combined with the carrier $(N)$ equation of the classic Rate Equations. Because the TWM is a distributed model each slice will have its own $N$ value and associated parameters (like gain and $\beta$, etc). This provides a flexible and detailed model compared to the lumped model of the REM. Along with the added detail comes the difficulty of accurately specifying all of the required parameters.

In addition to the TWM equations the laser model requires some kind of resonant cavity. The simplest one is using mirrors. This is because the TWM itself just provides a propagating medium that may have gain or loss. We require a resonant cavity for a laser as well as a gain medium for the laser to actually lase. The mirrors may be implemented internally to the TWM or externally as optical circuit elements. This will have consequences due to the different bandwidths of the TWM and the MNA engine. Running standalone (with the $N$ equation activated) eliminates the added complication of the MNA interface. The TWM resonant cavity was described in 5.9.

Note that even when run with the MNA engine the TWM must still be run as a separate simulation that propagates waves in a medium apart from the MNA engine. This is because it is an explicit implementation. A significant complication in doing so is the synchronization condition (see section 5.6) that must be upheld and requires the MNA engine and the TWM to have independent timesteps. The TWM is processing optical signals and so will typically have a higher bandwidth than the system MNA simulator. That means that for each timestep of the MNA engine a TWM simulation must be performed to advance the TWM time. Because the timesteps will not always be integral multiples of each other interpolation must be done. When the high-bandwidth signals of the TWM are passed out to the relatively low-bandwidth signals of the MNA engine the high frequency components are lost. This integration is covered in detail in [3]. It must still be integrated it with the carrier equation $(N \mathrm{DE})$ because we need to have the interaction with the carriers including the current source to model a laser.

The overall interaction of the TWM with the $N$ equation will be the same as in the REM, as it must be for both models to represent the same device. The difference is in the physical basis from which the photon density is generated in the TWM. Instead of a simple 
DE with growth and decay terms we have the physical medium with the forward and reverse electric fields in it. For the REM we use values averaged over the entire device and plug into the cross-coupled carrier and photon equations. In the TWM each slice has an average value that is plugged into its own cross-coupled equations. An extra step of propagating the traveling wave equations is necessary to calculate the new $E$-field. From that an average value of photon density can be calculate for $S$ to use in the cross-coupled equations. This is explained in the next section.

Many types of lasers can be modeled with the TWM. Distributed Feedback Lasers (DFBs) or Distributed Bragg Reflectors (DBRs) are suited to this model. See [34] for more information. Vertical-Cavity Surface-Emitting Lasers (VCSELs) are another possibility for the TWM. None of these are suitable for the REM. There are also interesting variations of lasers using the TWM such as Quantum Dot lasers. [35] Optical Injection is also of interest. [36] Before going more into the details of the traveling wave laser model it is important to note that the TWM is a building block that can be used in many optical systems or circuits, not just lasers. [37]

\subsection{Model Slice}

Each slice is very similar to the REM lumped model-average values for the cross-coupled equations. For the carriers the slices are treated as independent - it is assumed they do not interact with each other. This greatly simplifies the model. If desired one could add diffusion and charge distribution into the carrier flow. Preliminary investigation shows that these effects are minimal and can be ignored. [38]

The photon density, $S$, can be calculated from the forward and reverse envelope field equations to produce a value for the $N$ equation but the other direction-feeding the carrier density into the $S$ equation - is no longer a direct substitution. There is no longer an $S$ equation that we can insert $N$ into. To see this let's have a look at the basic lumped rate equations (3.12) and (3.13). They are reproduced here for convenience.

$$
\begin{aligned}
\frac{d N}{d t} & =\frac{I}{e V}-g_{0}\left(N-N_{t r}\right) S-\frac{N}{\tau_{n}} \\
\frac{d S}{d t} & =\Gamma g_{0}\left(N-N_{t r}\right) S-\frac{S}{\tau_{p}}
\end{aligned}
$$

Here is the TWM distributed $N$ equation along with the first-order traveling wave equations (5.1) and (5.2) as well:

$$
\begin{gathered}
\frac{d N(z, t)}{d t}=\frac{J}{e W H}-g_{0}\left(N(z, t)-N_{t r}\right) S(z, t)-\frac{N(z, t)}{\tau_{n}} \\
\left(\frac{\partial}{\partial t} \pm v \frac{\partial}{\partial z}\right) E_{ \pm}(z, t)=-i v \beta E_{ \pm}(z, t)
\end{gathered}
$$




$$
\beta=\frac{1}{2}\left[\delta-i \alpha_{b}+\left(i+\alpha_{H}\right) g\right]
$$

If we recognize that the gain $g$ in (6.5) is dependent on $N$ just as it is in the REM then we can make the following substitution for $g$

$$
g=\Gamma g_{0}\left(N-N_{t r}\right)
$$

Therefore $\beta$ in the TWM is dependent on $N$ and will determine the electric field solution. This is in turn used to calculate the photon density and so the TWM replaces the photon density DE and is cross-coupled with the $N$ equation as desired. In a full MNA circuit simulation the photon density is calculated over the length of the active layer and then averaged to give the model result. Seeing how closely these two models are related one can imagine that the REM could be derived from the TWM. This is explored in appendix I.

If we think of the $z$-domain we have a number of slices where each slice has it's own $N$ value at each time $N_{k}^{n}$ where $n$ represents discrete time and $k$ discrete space in $z$. Each slice evolves on its own but is affected by the waves propagating through it and it's current value of $N$. Note that $\beta$ is a function of $N$ and is changing potentially every step.

If we look at the discretized equation for $E$ (forward traveling wave)

$$
E_{k}^{n}=E_{k-1}^{n-1} e^{-i \beta_{k}^{n-1} \triangle z}
$$

we see that it depends on previous time values of $E\left(E^{n-1}\right)$ as well as neighbouring $z$ values of $E\left(E_{k-1}\right)$; in this case the value where the field just was. It also depends on the previous value of $\beta\left(\beta^{n-1}\right)$ for the current slice $k$. $\beta$ itself is dependent on the gain

$$
\beta_{k}^{n-1}=\frac{1}{2}\left[\delta-i \alpha_{b}+\left(i+\alpha_{H}\right) g_{k}^{n-1}\right]
$$

which itself is dependent on $N$

$$
g_{k}^{n-1}=g_{0}\left(N_{k}^{n-1}-N_{t r}\right)
$$

When run with the MNA engine in circuit mode with its implicit implementation it will solve a system of matrix equation for each slice. Between each system time step the TWM will have to march time with its explicit traveling wave equations. In these steps the slices are dependent on each other but after $E$ is updated an average slice value is calculated to pass out to the circuit. Note that there will not necesarily be an integral number of steps in the TWM simulation for every system time step so interpolation will have to be done when moving between the system and TWM time domains [3]. Typically this is not a problem as the bandwidth of the TWM will be much higher.

\subsubsection{Propagation Examples}

We can now compare a simple case between the TWM and the TWM laser with the carrier $(N)$ equation. We will inject an optical signal into the active layer and let it propagate. In 
the case without the $N$ equation, if we set $\beta=0$ we will get no gain and the signal will just be delayed. An example of this is shown in figure 5.1. The example has some modulation but it is clear that the signal is only delayed. For the TWM laser we will eliminate the resonant cavity (by removing the right-hand side mirror) so that we see the propagation as it is affected by $N$. We will start $N$ at transparency and then with a current step we will see the gain increase with $N$ over time eventually reaching a steady state value. At first there will be no gain just as in the standalone simple propagation simulation but then it will slowly increase to the steady state value. This is shown in figure 6.1. As the signal propagates through the
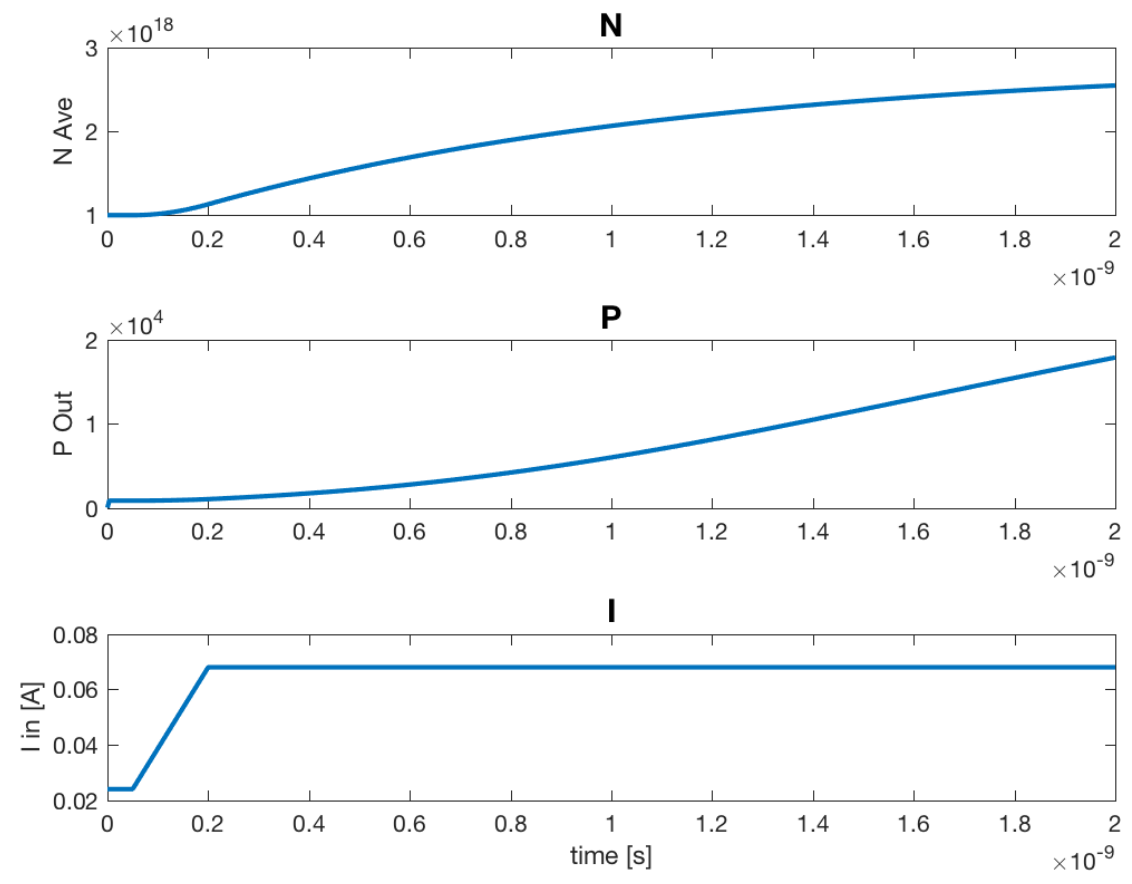

Figure 6.1: Optical signal (const amplitude) propagating through traveling wave laser model. Right reflector has been removed. Starting from transparency the current step supplies carriers until the stimulated emission is sufficient to establish an equilibrium. a) Carrier density. b) Photon density. c) Current step.

active layer the stimulated emission will cause the signal to be amplified and this in turn will consume carriers. So as expected we see in figure 6.2 the carrier distribution drops off with increasing $z$. We note that it appears to be an exponential decline, but at this length of device the change is not significant. We can also set the static gain of our standalone simple propagation equal to the average of the model with the distributed $N$ and compare. This is shown in figure 6.3. We see over the length of the models they behave almost identically. We expect the model integrated with $N$ to have less gain as $N$ drops with $z$ but the arbitrary gain factor we set for the simple propagation case was chosen to match the average gain 


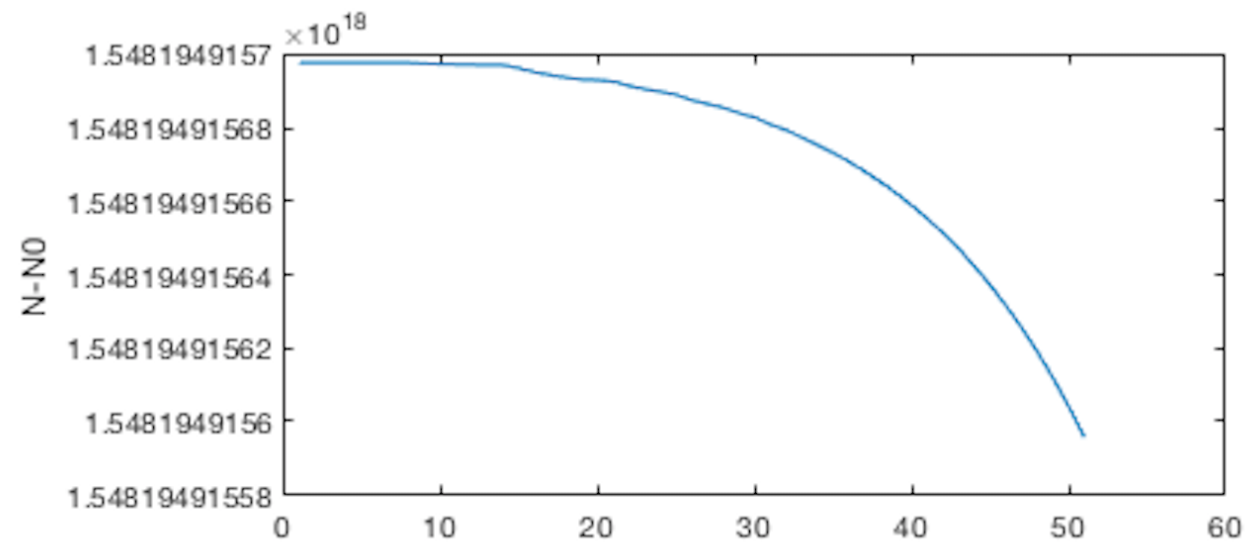

Figure 6.2: Carrier distribution of optical signal propagating through traveling wave laser model. Carrier concentration in $\mathrm{cm}^{-3}$ versus laser segment number $(z)$.

over the active layer. This shows how a lumped model with fitted parameters can produce the correct result. The distributed model would hopefully be able to determine the correct result. On the right side of the figure showing the models over time, however, we see the very different nature of the two models. The model without $N$, after a very short time delay, reproduces the input on the output. The model with integrated $N$ takes a relatively long time to build up the carrier density so there is gain from the stimulated emission. This in turn reduces the carriers. Again the distributed model gives a better match to the actual laser, even though the net effect may be similar.
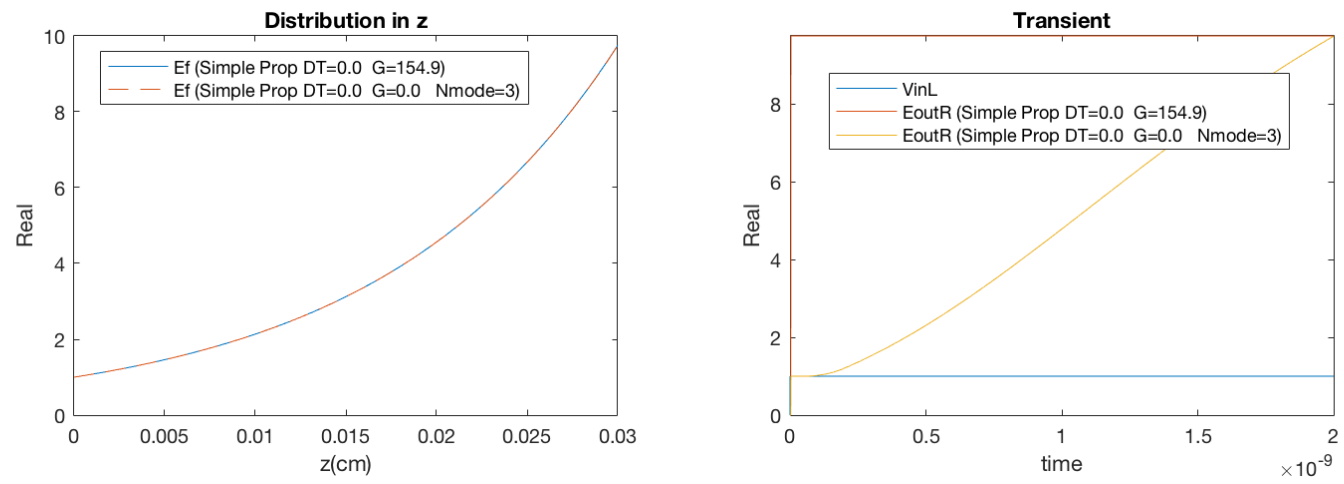

Figure 6.3: Comparison of traveling wave models: Without $N$ and integrated with $N$.

\subsection{Field Distributions}

Running now with a normal laser with a resonant cavity with mirrors at both ends we look at the field distributions. A typical carrier distribution is show in figure 6.4. This is over the 
physical extent in the $z$ direction of the medium. Its symmetry makes sense as the waves will bounce back and forth off the end mirrors. As the photons propagate, the stimulated emission causes the photon density to increase but consumes carriers as it goes. If it were only from one direction we would expect $N$ to decay (as above). Removing the right-hand mirror we see in figure 6.5 that the $N$ density drops as the wave propagates from left to right. But because the laser cavity (figure 6.4) is symmetrical it decays in both directions simultaneously. When we superimpose these opposite-traveling waves on one another we get the symmetrical shape as shown.

An interesting note on this distribution is that because $N-N_{t r}$ is not the same throughout the active layer, even if the index $n$ is assumed constant for the entire layer, the effect of $\beta$ will be different for each slice.

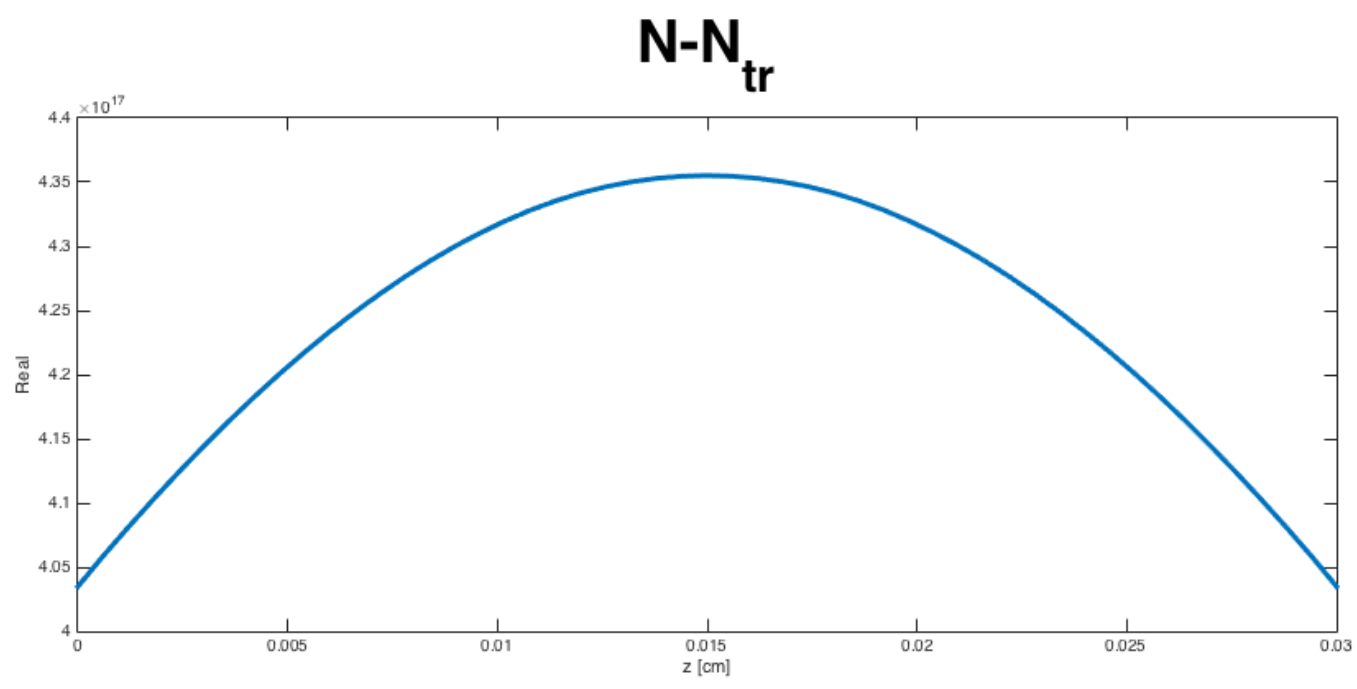

Figure 6.4: TWM carrier distribution 


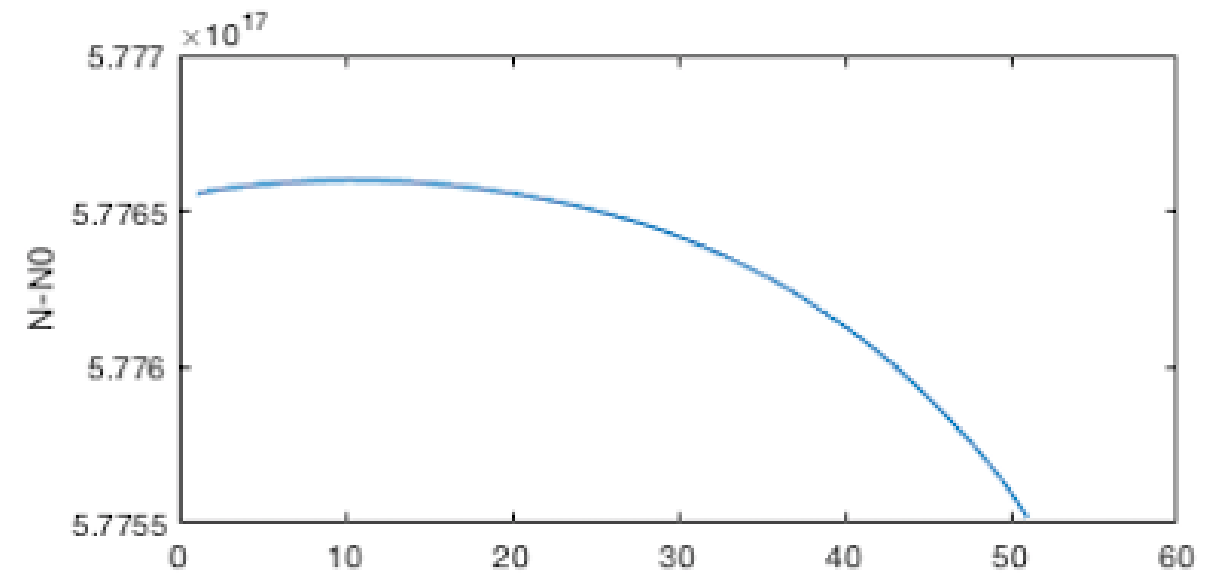

Figure 6.5: TWM carrier distribution after the right-hand mirror has been removed. 


\section{Chapter 7}

\section{Comparison of TWM with REM}

The REM has been remarkably useful for modeling laser dynamics in a standalone simulation. The overall behaviour can be reasonably accurate by fitting the parameters to the measured device. Because of the distributed nature of the TWM there is great potential that it can provide a much more detailed laser model than the REM. Of course with increased detail - and hopefully accuracy - comes an extra cost. Do the benefits outweigh this added cost?

With the distributed model there are many aspects that are no longer lumped into one number for the model. The fields can now be calculated for the entire extent of the device. This means that the photon density is known throughout the device giving much more information on local conditions of field strength within the material. The carrier density can be known in greater detail. This makes it possible to query carrier diffusion in the device and other phenomenon such as hole burning. Hole burning is where increased photon density due to a mode being present will deplete that physical area of carriers. Once this happens stimulated emission in that localized area will cease. We see a similar effect because of the way the carrier density is distributed over the length as in figure 6.4 where the gain which is proportional to $N-N_{t r}$ will not be a constant over the length. At the ends of the device due to the low carrier density we see that the gain may even switch sign. The distributed model not only gives us a better estimate than a lumped model but lets us see the impact of the carrier distribution, photon density and any other parameter that is not constant over the device.

\subsection{Carrier and Photon Densities}

First we will start both models at transparency and compare the carrier and photon densities. We start at transparency $\left(N_{t r}\right)$ because at this carrier density there is no gain and so there will be no changes in the system state. For the REM we start with the single-mode model. This is shown in figure 7.1 and shows that the carrier and photon densities agree in the basic dynamics and levels between the two models. Note that there is a delay in the TWM. There is also a slightly longer time between pulses of the TWM. This could explained by 
the physical nature of the model - it takes time to propagate the optical signal through the model. Unfortunately the delay does not correspond to the time delay through the active layer. A possibility is that it is due to the time it takes for all segments of the laser to begin to lase which will be longer than the REM. This would be expected to produce a more damped response for the TWM which is not what what we see. It is a difference created by the distributed versus lumped models but more investigation is needed. This will also thought to explain the longer time between the pulses of the TWM as well.

Figure 7.2 shows the spectrum of the output. Both models have the same fundamental mode but the TWM has many more modes. This is because in the TWM the modes emerge from the model and so all excited modes will be present. In the REM the modes are set by the user so only a finite number will exist. In the case of single-mode that is one.
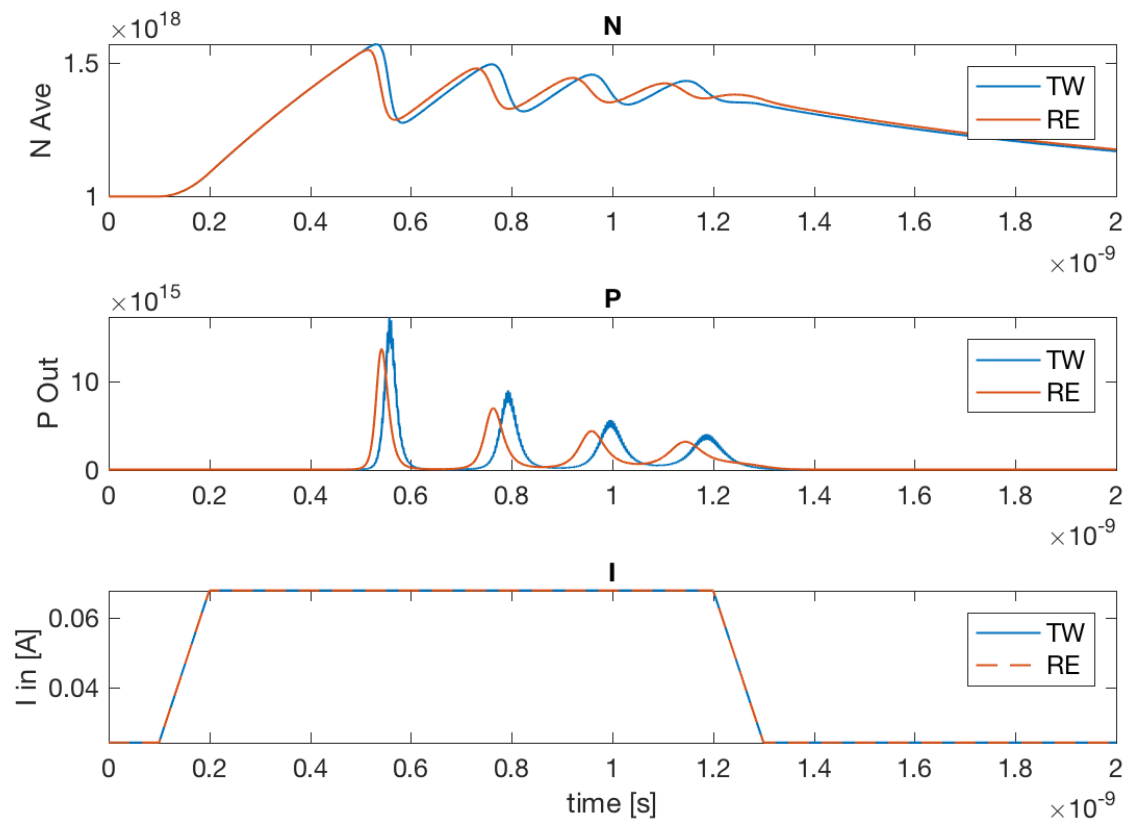

Figure 7.1: REM single-mode and TWM laser models turn on during current pulse showing carrier and photon densities and the current pulse.

Let's now have a look at the more advanced multi-mode REM. The first thing to note is that with this model we first came up against the problem of mode-locking as discussed in section 3.6. This was corrected by generating a random phase for each mode. The TWM on the other hand relies on spontaneous emission to generate the modes. This is an example of how physical the TWM is. Presumably there are affects that will be modeled correctly before they are even known. This is a great advantage of a physical model.

With the multi-mode REM we chose 11 modes. We see in figure 7.3 that the carrier and photon densities again agree. They do not change much as the modes in REM are increased. In figure 7.4 the spectrum of the multi-mode again agrees up to the number of modes we set 

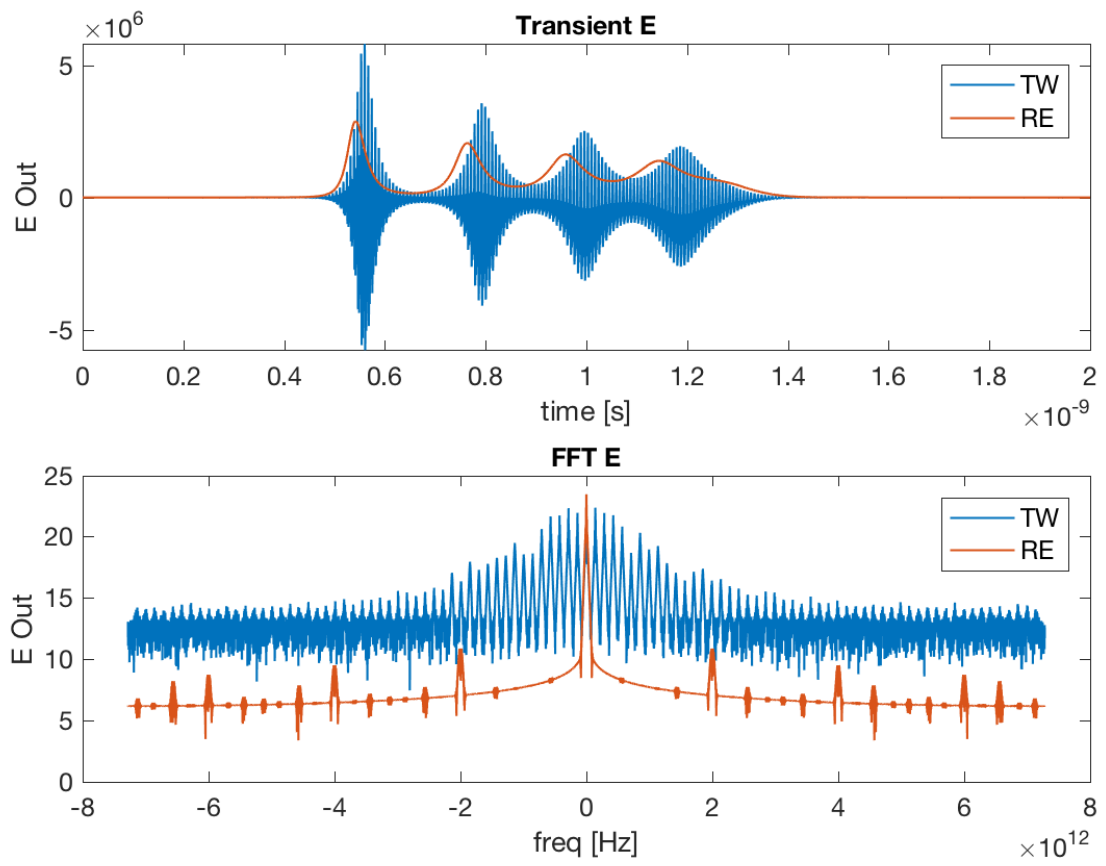

Figure 7.2: REM single-mode and TWM laser models turn on showing E-field and FFT after laser models turn on.

in the REM. The shape of the spectrum also agrees but this is not surprising as we set the Lorentzian the same in both models. 

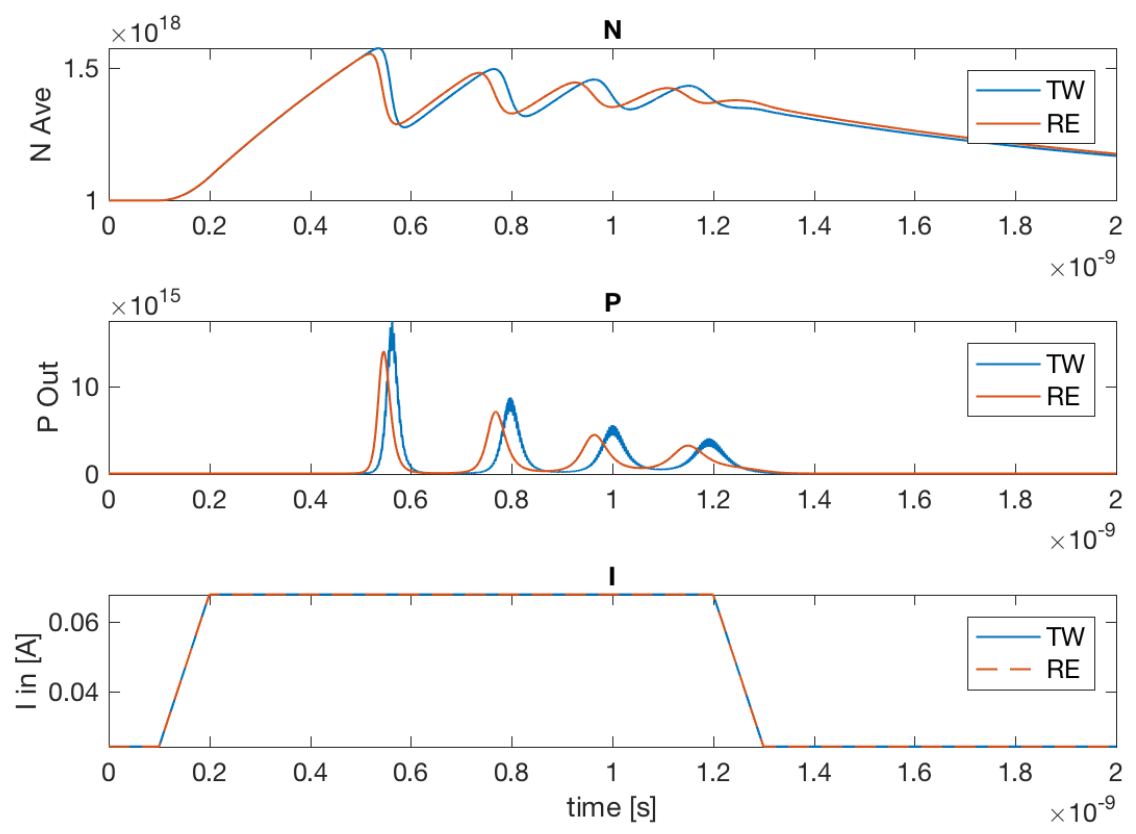

Figure 7.3: REM multi-mode with 11 modes and TWM laser models as they turn on with a current pulse. 

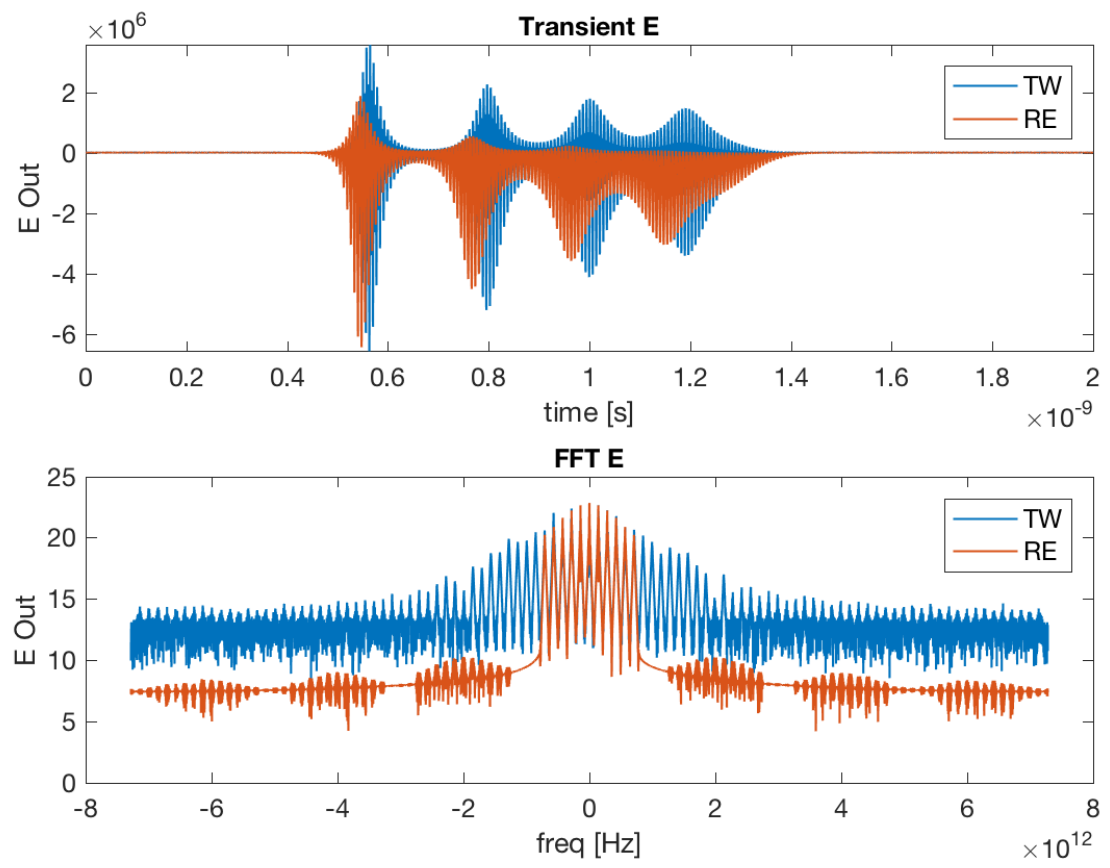

Figure 7.4: REM multi-mode with 11 modes and TWM laser models showing FFT after laser models turn on. 


\subsection{Phase}

Let's take a closer look at the phase of these two models. If we look back at figures 3.12 and 3.13 we see that the REM calculates the phase for each mode separately. When added together we will get the output signal phase. Note that the carrier density $N$ and the photon density $P$ are basically the same for both the 3-mode and 11-mode cases. This is true for all numbers of multi-modes tested. If we add the modes and create the output $E$-field we get the result in figure 7.5. We see that the phase of the modes have cancelled out and the $E$-field remains real. This will be the same for every run as there is no random element.
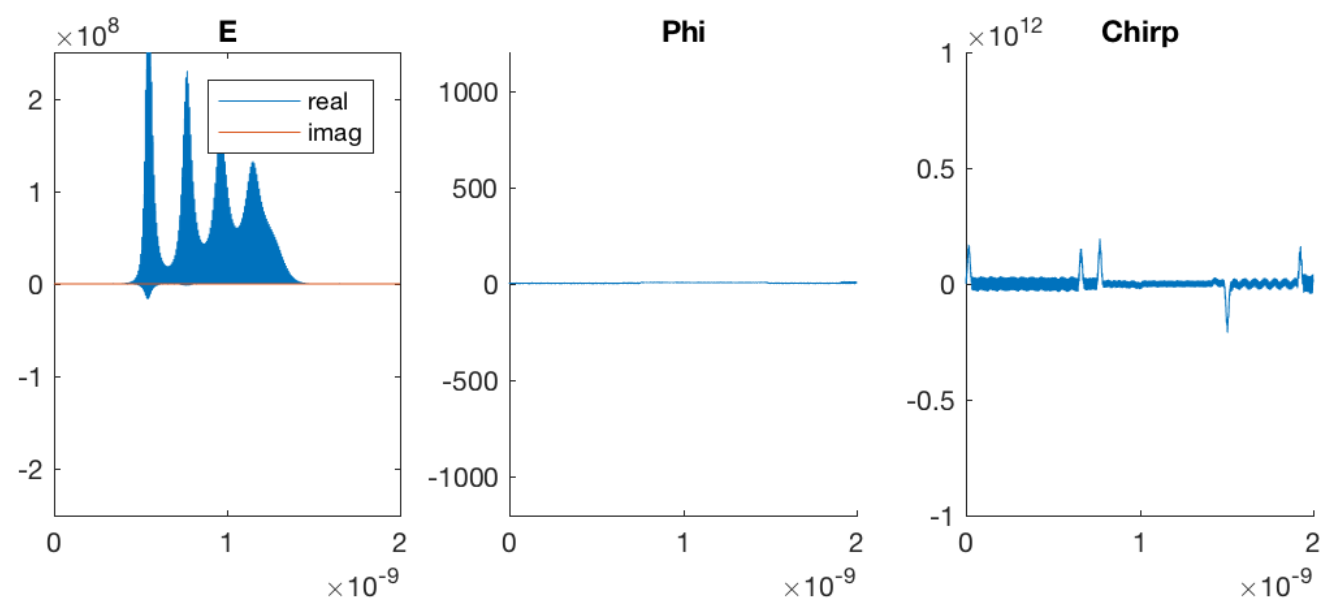

Figure 7.5: E-field, phase and chirp for REM 11-mode simulation with initial phase of zero for each mode.

If we set an initial random phase for each mode (to prevent mode-locking as discussed in section 3.6) and run the simulation we get the results in figure 7.6. This shows two runs of the same simulation and even though it appears as if all modes start with a phase of zero they are actually random between $(0,2 \pi)$; it is just the large scale that masks this. What we see is that even though the phase of the individual modes is well behaved (linear) the sum fluctuates in an almost chaotic manner. An investigation was done to determine what makes the overall phase seem chaotic. Notice that the phase changes when the laser current pulse is turned on and off - the slope of the phase changes here. This is when the laser is lasing. The first plot shows one random initial phase with the modes summed while the second plot (below) shows five runs with random initial phases for each mode for the same simulation. With 11 modes we see a distinct difference when the current is on. The behaviour is best understood with amplitude configurations. Before lasing the amplitudes are in a random configuration with no clearly dominant modes. During lasing the fundamental mode has most of the power with the next side modes significant. In the non-lasing configuration the phase changes with a randomly selected mode while in lasing mode it tends to follow the fundamental. This is best explained using phasors to represent the modes' amplitudes and phase. If the phasors all have roughly the same amplitude and random phase some 
configurations will cause the total of the phasors to continually circle the origin (building up phase). When the fundamental is largest and they then decrease the total phase will not be able to wrap around the origin and accumulate phase. This is explained in detail in appendix $\mathrm{C}$.
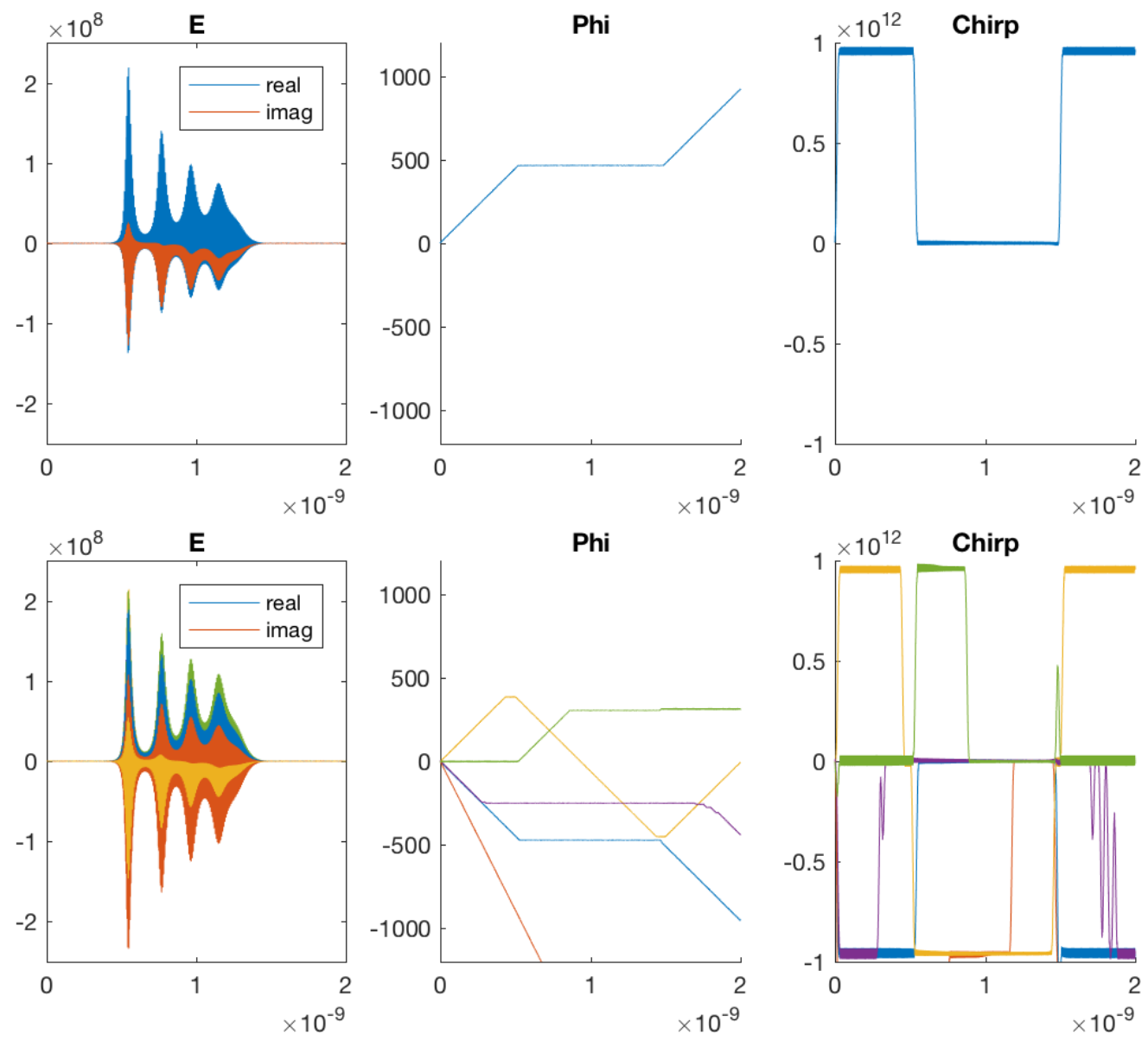

Figure 7.6: E-field, phase and chirp for two runs of the same 11-mode REM simulation. Each run starts with a random initial phase on each mode. This first plot shows one random initial phase while the second plot shows five runs each with different random initial phases for each mode.

If we run the REM in single-mode this chaotic phase phenomenon does not occur even when we start the fundamental mode with random phase. Figure 7.7 shows two single-mode runs starting both without and with random phase. In the $E$-field plot we can see the different phase between the two runs but the phase and chirp plots both remain at zero. Because we only have the fundamental mode with the carrier chosen to be the same there will be no accumulation of phase as with higher modes.

The TWM also exhibits randomness in the phase as well. In figure 7.8 we see the TWM phase changing in a random fashion at the beginning and end of the simulation. The REM 

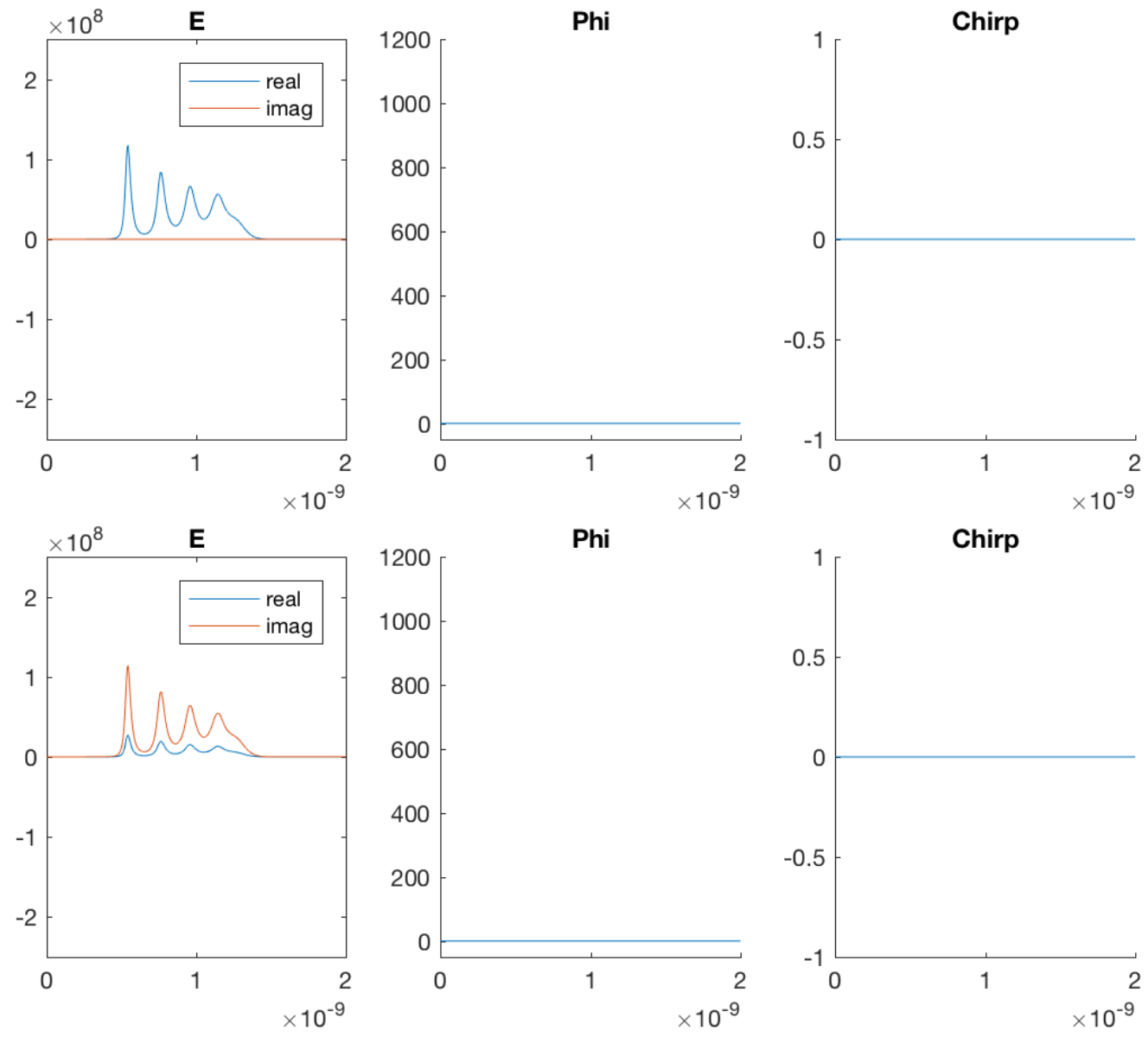

Figure 7.7: E-field, phase and chirp for single-mode run of REM starting without random phase (top) and starting with random phase (bottom).

phase is not changing because it is a single-mode simulation. Refer back to figure 7.6 for the random changing of the REM phase. In the TWM, however, the random change in phase is due to the spontaneous emission from which the optical lasing signal grows. In a laser there is always spontaneous emission even when there is no stimulated emission. It is from this spontaneous emission that a population inversion will create stimulated emission. The TWM behaves like the real laser and if the carrier density gets large enough it will create stimulated emission. For spontaneous emission we use a Monte Carlo technique so will have random phase. If we did not model the spontaneous emission the TWM would not be able to generate all of the modes - only those already present in the signal. Once the laser is lasing the stimulated emission overwhelms the spontaneous emission, which is still present, and its random phase will be negligible. So we expect random phase in the TWM until the laser crosses threshold. Once the laser is dominated by stimulated emission the 'phase configuration' of the field is frozen and we have only a gain present. This is clearly seen in the figure - the TWM phase is constant once the current (pump) is turned on and the laser 
crosses threshold. Whatever phase the laser reaches at threshold is held and the field is then only amplified as it cycles back and forth between the mirrors. Once the current is shut off the population inversion is lost, the gain falls, and the spontaneous emission with its random phase becomes significant again. This random phase makes it difficult to compare with the phase of the two models but does explain the behaviour of the TWM and demonstrates its physical nature. It is important to remember that so far the Henry factor has been kept at zero so all REM phase changes have been due to the adding of different frequency signals (the modes).
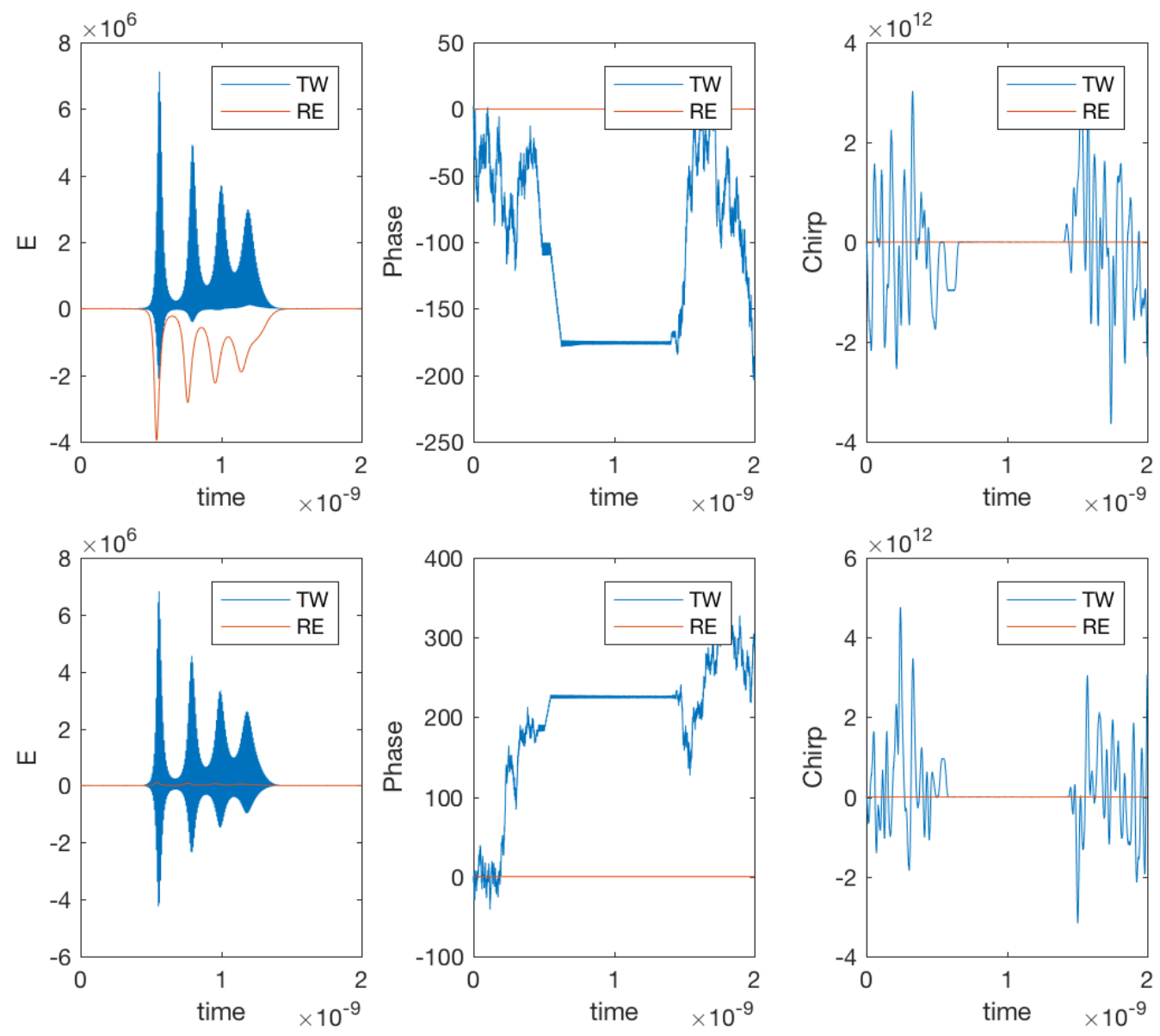

Figure 7.8: E-field, phase and chirp for two runs of single-mode REM compared with TWM. Henry factor set to 0 and $N$ starting at transparency.

\subsection{Henry Factor}

The Henry factor is closely related to the phase. In the REM it only affects the calculation of phase. See (3.11) for example. The TWM is not so simple but as we will see it will affect 
the detuning and therefore the phase. First let's look at the REM. Referring to figure 7.9 the carrier density is not affected by the Henry factor as again, the Henry factor only appears in the phase equation. When a Henry factor of 5 is set we see the slopes of the phases increase indicating a shift of frequency or chirp. This is hard to see in the $E$-field plot but a zoom in of the $E$-field of one of the modes in the lower plot shows that the frequency has increased with the Henry factor.
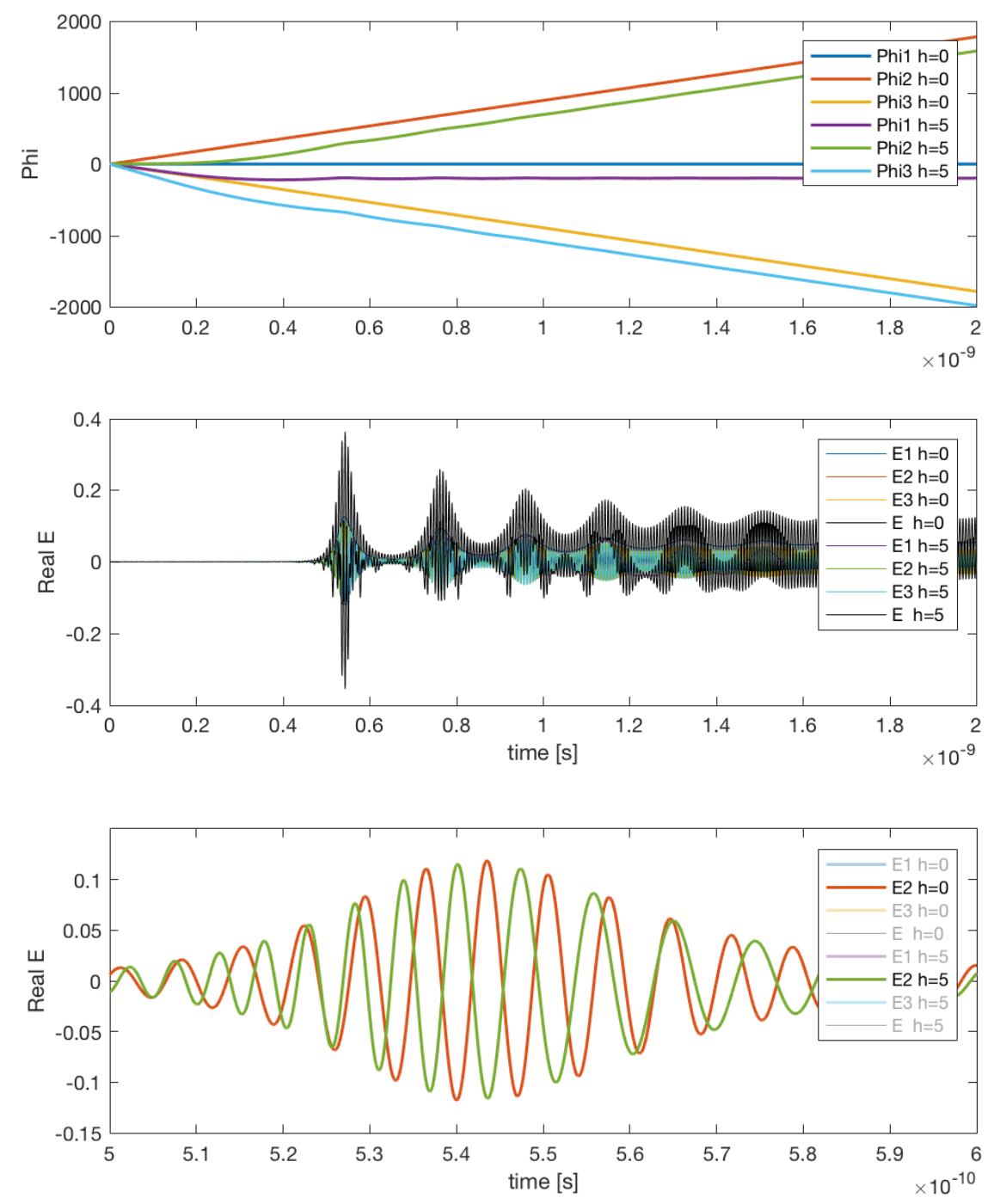

Figure 7.9: Comparison of REM simulation with Henry factor of 0 and 5. Carrier density $N$, Photon density, phase and $E$-field. A zoom in on the $E$-field for the second mode is shown in bottom plot where the frequency shift is apparent.

Comparing the REM with the TWM, figure 7.10 shows the carrier and photon densities for the REM and TWM for two values of the Henry factor. As before (figure 7.3) the two 
models match and show the Henry factor has little effect on the carrier and photon densities in either model.
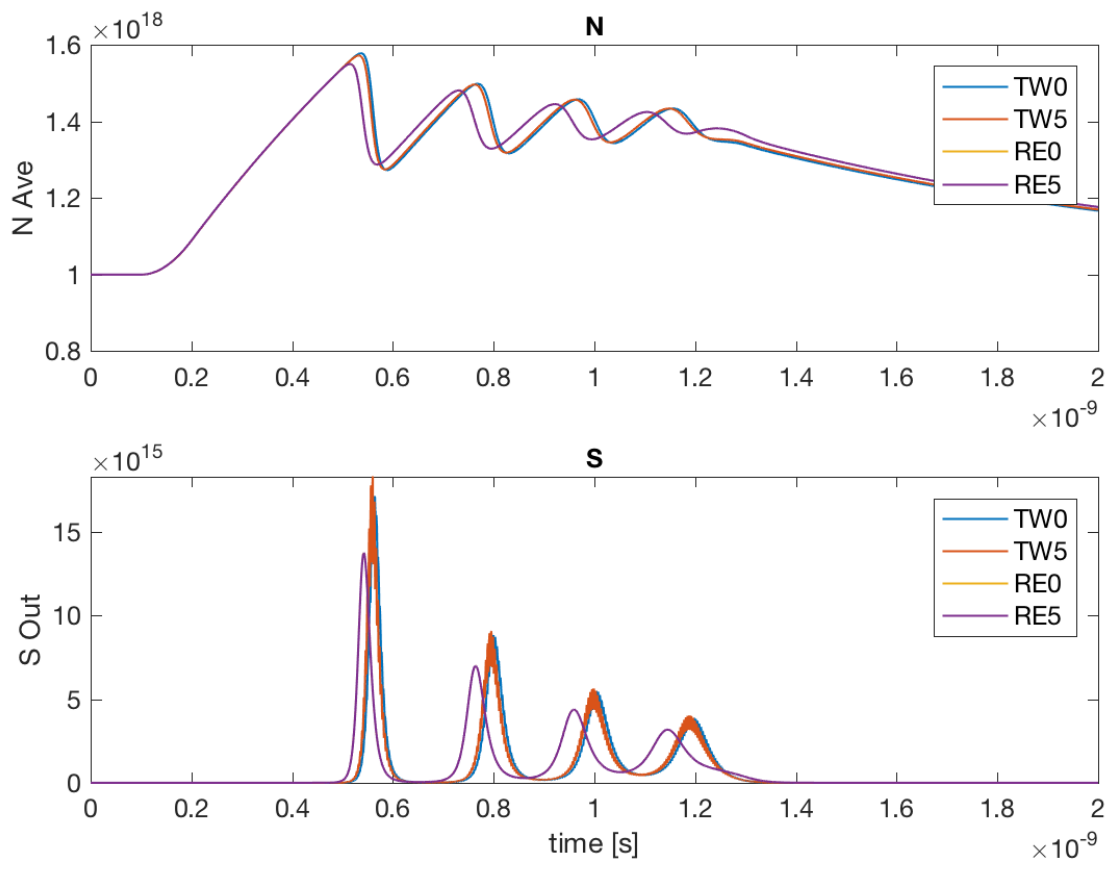

Figure 7.10: Carrier density $N$ and photon density with Henry factor of 0 and 5 for 3-mode REM compared with TWM.

In figure 7.11 we compare the single-mode REM with the TWM for a Henry factor of 5. As in figure 7.7 the REM phases of each run are the same because in single-mode the REM phase does not appear chaotic by randomizing its initial phase as in the multi-mode case. With a Henry factor they are still the same and the chirp (derivative of phase) is now linearly related to the $N$ of figure 7.10. The two TWM runs are not the same either because the modes are generated by noise and will be different every run. We do, however, see a striking similarity in the chirp during the current pulse (center of simulation) as both models exhibit a chirp linearly related to $N$.

Similar results are obtained when the multi-mode REM is run with 3 modes. Simulations with the Henry factor set to 0 and 5 are shown in figures 7.12 and 7.13 respectively. We see again without a Henry factor the stable phase during lasing and the fluctuating chirp of the TWM when not. In these particular examples the agreement between models is excellent. With the Henry factor we get the clear $N$ characteristic in the chirp.

When the current pulse is off the TWM simulation is very noisy and this is because when the laser is not lasing the phase will be determined completly by the spontaneous emission or noise. It is random (as seen at the beginning and end in the Phase plots) and therefore the chirp, being its derivative, will be even noisier. We should be able to eliminate the noise when the current is off by starting the laser at threshold instead of transparency. Figure 7.14 

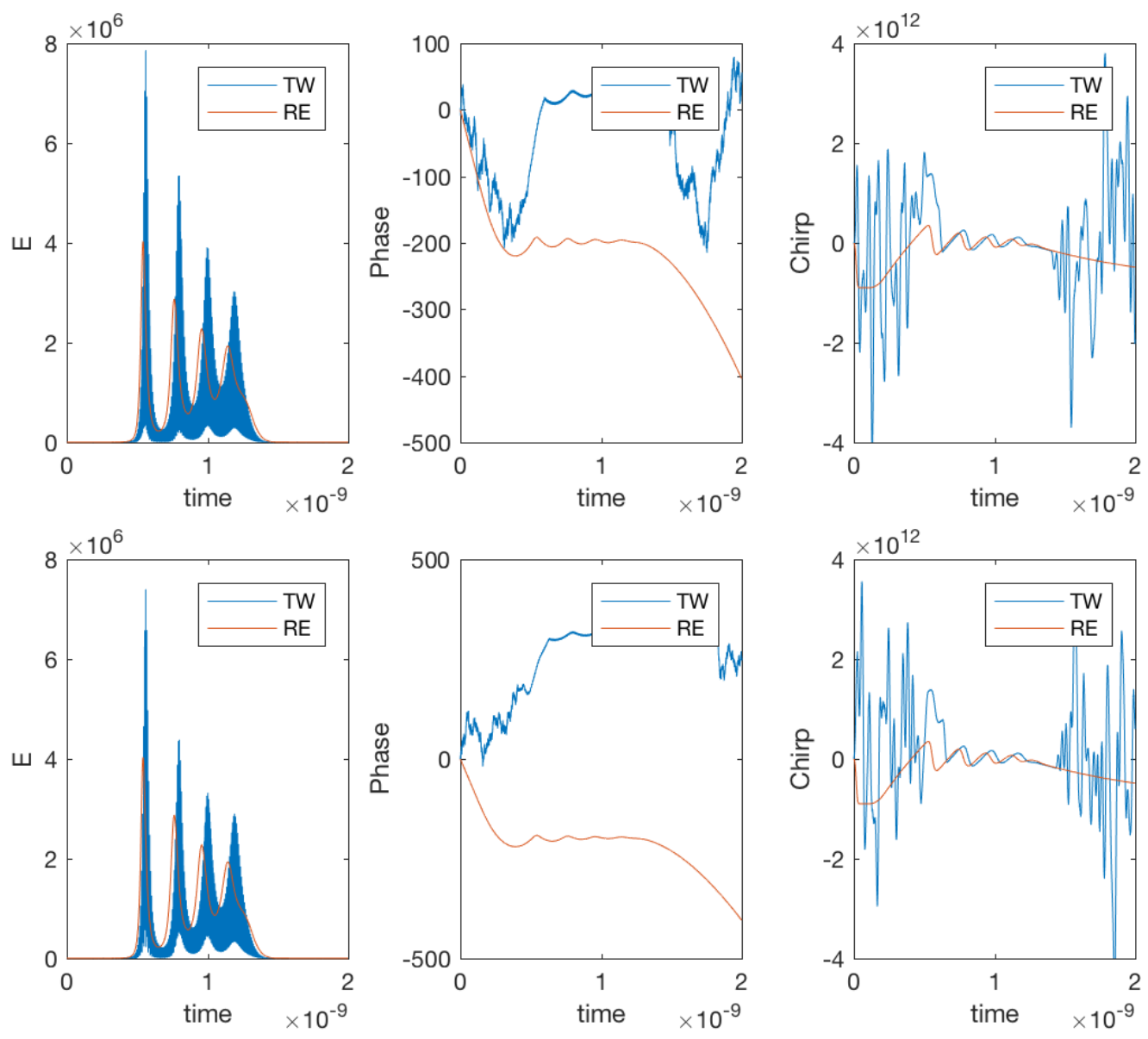

Figure 7.11: E-field, phase and chirp for two runs of single-mode REM compared with TWM. Henry factor set to 5 and $N$ starting at transparency.

shows the carrier and photon densities for the REM and TWM for two values of the Henry factor with the carrier density starting at threshold and a current step applied. This keeps the laser on the entire simulation. As before (figure 7.10) the two models are a reasonable match and the Henry factor has no effect on the carrier and photon densities.

Figures 7.15 and 7.16 show a comparison of the REM and TWM for Henry factors of 0 and 5. The REM simulation was single-mode. With the Henry factor set to 0 the REM phase is constant and the chirp is held at 0 by the Henry factor. The TWM phase is now quieter because we are above threshold.

Once the Henry factor is enabled we see the phases of both models change (figure 7.16). The chirp for the REM is now linearly related to $N$ as before. The TWM chirp is remarkably similar.

The comparison is repeated for the 3-mode REM and the TWM and is shown in figures 7.17 and 7.18. There is not much difference from the simulations of the single-mode REM.

One significant difference between the two models that we have yet to mention is the 

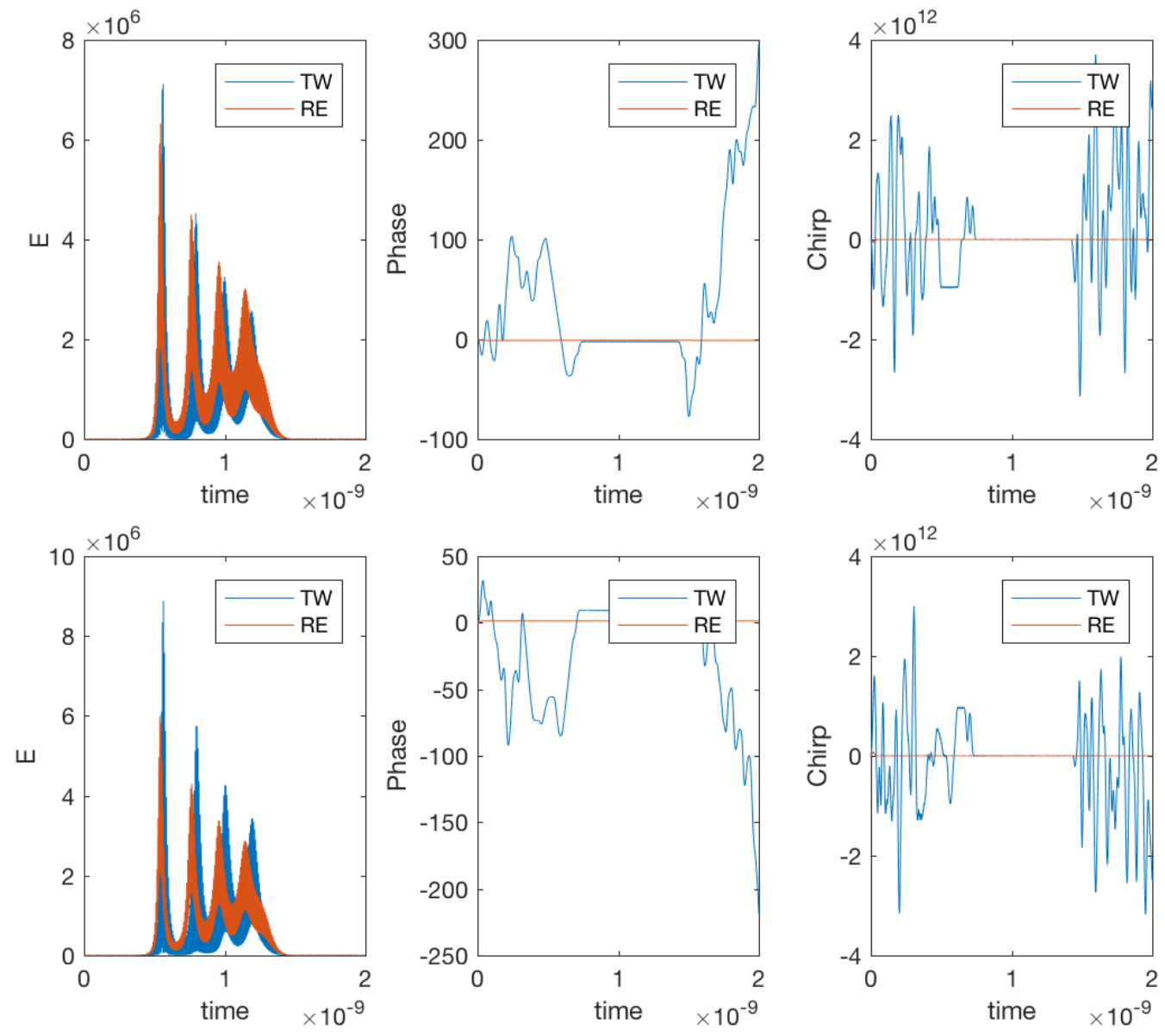

Figure 7.12: E-field, phase and chirp for two runs of 3-mode REM compared with TWM. Henry factor set to 0 and $N$ starting at transparency.

steady state that the two models reach. The REM settles on a constant phase and so the chirp goes to zero. The TWM phase on the other hand continues to grow linearly producing a constant positive chirp. This is because the TWM is inherently set to have zero chirp at transparency and when we increase $N$ to threshold we will have a significant chirp. This will always be there whenever there is a Henry factor.

To see this let's rewrite the TWM $\beta$ equation (5.2) and collect the real and imaginary parts so the effect of the Henry factor is more obvious.

$$
\beta=\frac{1}{2}\left[\delta+\alpha_{H} g+i\left(g-\alpha_{b}\right)\right]
$$

The Henry factor adds a real part to $\beta$ which from section 5.3 we know causes the field to rotate and change spatial frequency. If we express $g$ as a function of $N, g=g_{0}\left(N-N_{t r}\right)$, and separate the real and imaginary we get

$$
\beta_{r}=\frac{1}{2}\left[\delta+\alpha_{H} g_{0}\left(N-N_{t r}\right)\right] \quad \beta_{i}=\frac{1}{2}\left[g_{0}\left(N-N_{t r}\right)-\alpha_{b}\right]
$$



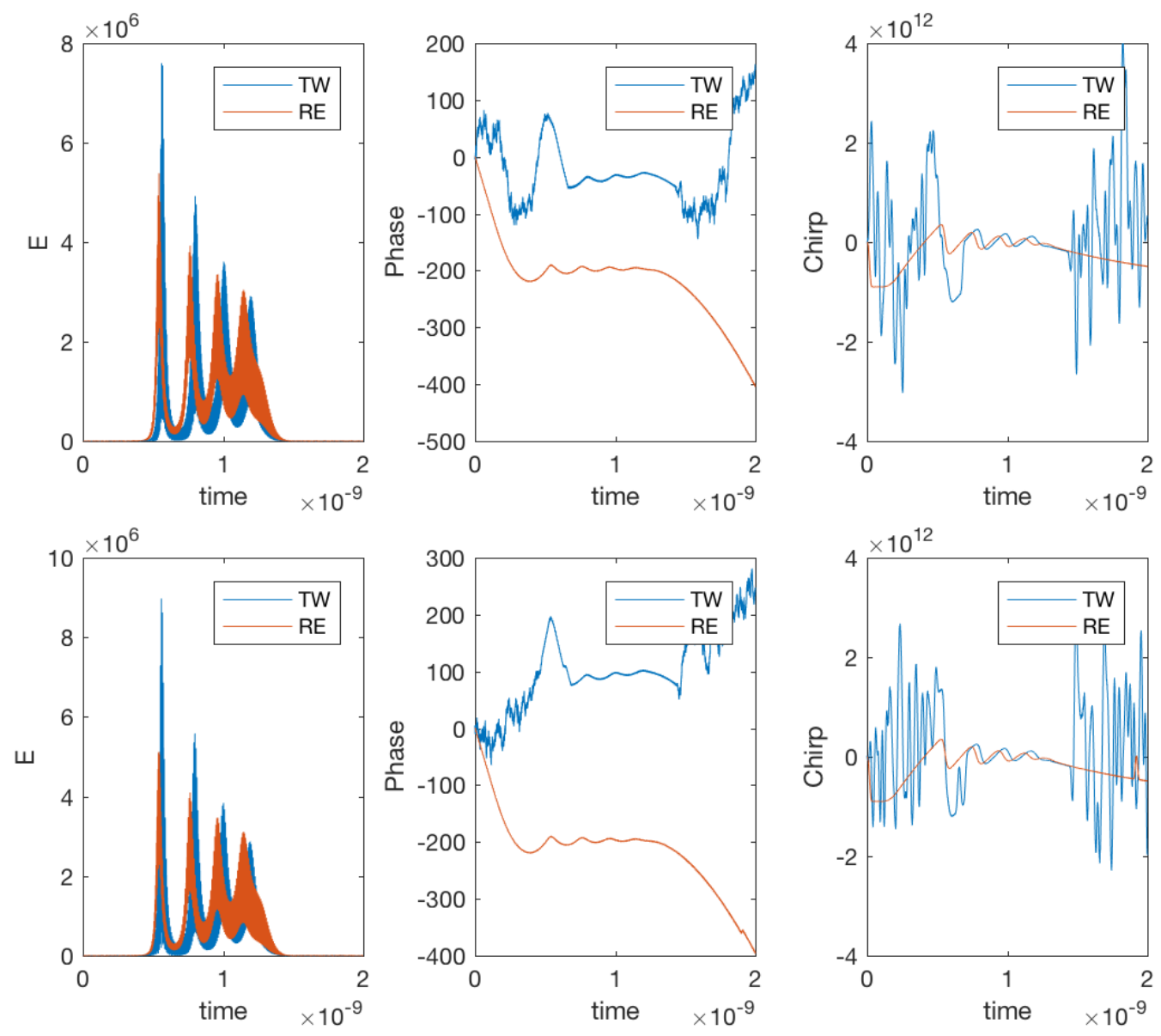

Figure 7.13: $E$-field, phase and chirp for two runs of 3-mode REM compared with TWM. Henry factor set to 5 and $N$ starting at transparency.

Again from sections 5.3 and 5.4 we know that the real part is the detuning while the imaginary part is the gain. Now from (7.2) we can see why a Henry factor of 0 eliminates any detuning and the $N$ dependent gain controls the TWM gain regardless of the Henry factor. Once the Henry factor is zero the only term left in the real part of $\beta$ is the static detuning $(\delta)$. But when non-zero the Henry factor will add to $\beta_{r}$ and detune the laser. Because of the dependency on $N$ it will also be modulated by $N$. Also from (7.2) we see that at threshold $g=g_{0}\left(N_{t h}-N_{t r}\right)$ and the chirp will be positive because the added detuning will be

$$
\beta_{r}=\frac{1}{2} \alpha_{H} g_{0}\left(N_{t h}-N_{t r}\right)
$$

From (5.11) we see that the amount of rotation or change in spatial frequency or chirp is actually the real part of $\beta$. This means $\beta_{r}$ above is the chirp. 

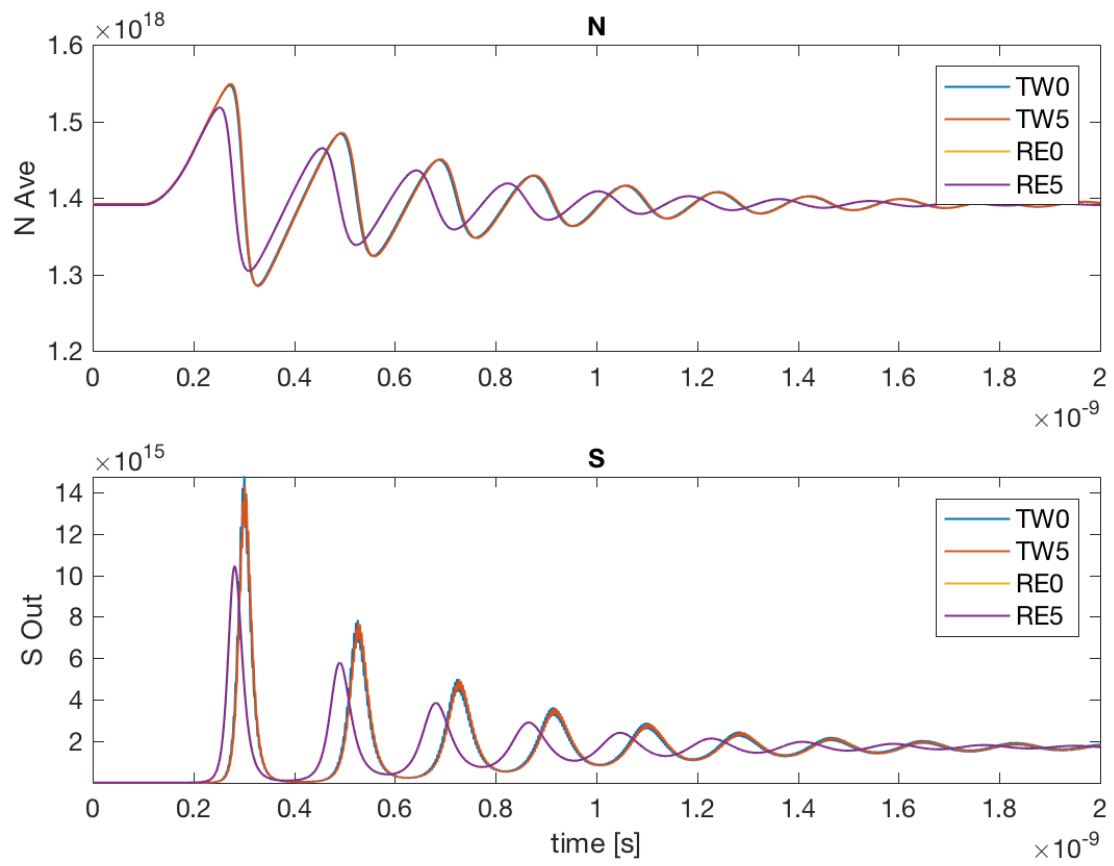

Figure 7.14: Comparison of simulation with Henry factor of 0 and 5 for single-mode REM and TWM showing carrier density $N$ (starting at threshold) and photon density. The plots are almost identical for the 3-mode REM. Note the current is a step.

From the REM phase equation (3.11) reproduced here

$$
\frac{d \phi}{d t}=\frac{\alpha_{H}}{2}\left[\Gamma g_{0}\left(N-N_{t r}\right)-\frac{1}{\tau_{p}}\right]
$$

we see that the chirp will be zero at threshold. To set the chirp of the TWM to zero at threshold we need to add a static detuning term. If we use the REM to estimate it we know that at threshold we have

$$
\Gamma g=\frac{1}{\tau_{p}}
$$

so for the TWM we must have

$$
\begin{array}{r}
\frac{1}{2}\left[\delta+\alpha_{H} g\right]=0 \\
\delta=-\frac{\alpha_{H}}{\Gamma \tau_{p}}
\end{array}
$$

The previous runs had the detuning set automatically by the above equation (7.6). The value is not exact as it was obtained from the REM and does not include distributed effects. 
By adjusting this parameter we can set the threshold chirp to exactly zero. This is shown in figures 7.19 and 7.20. Note when the Henry factor is zero (figure 7.19) the detuning factor produces a negative slope and chirp. The number of REM modes will not affect the TWM phase.

The models compare nicely. When it comes to any details it is not clear which of the models will be correct. We know that some of the REM characteristics (like mode-locking) are not. We expect that the TWM is more accurate but this must be left for experiment. 

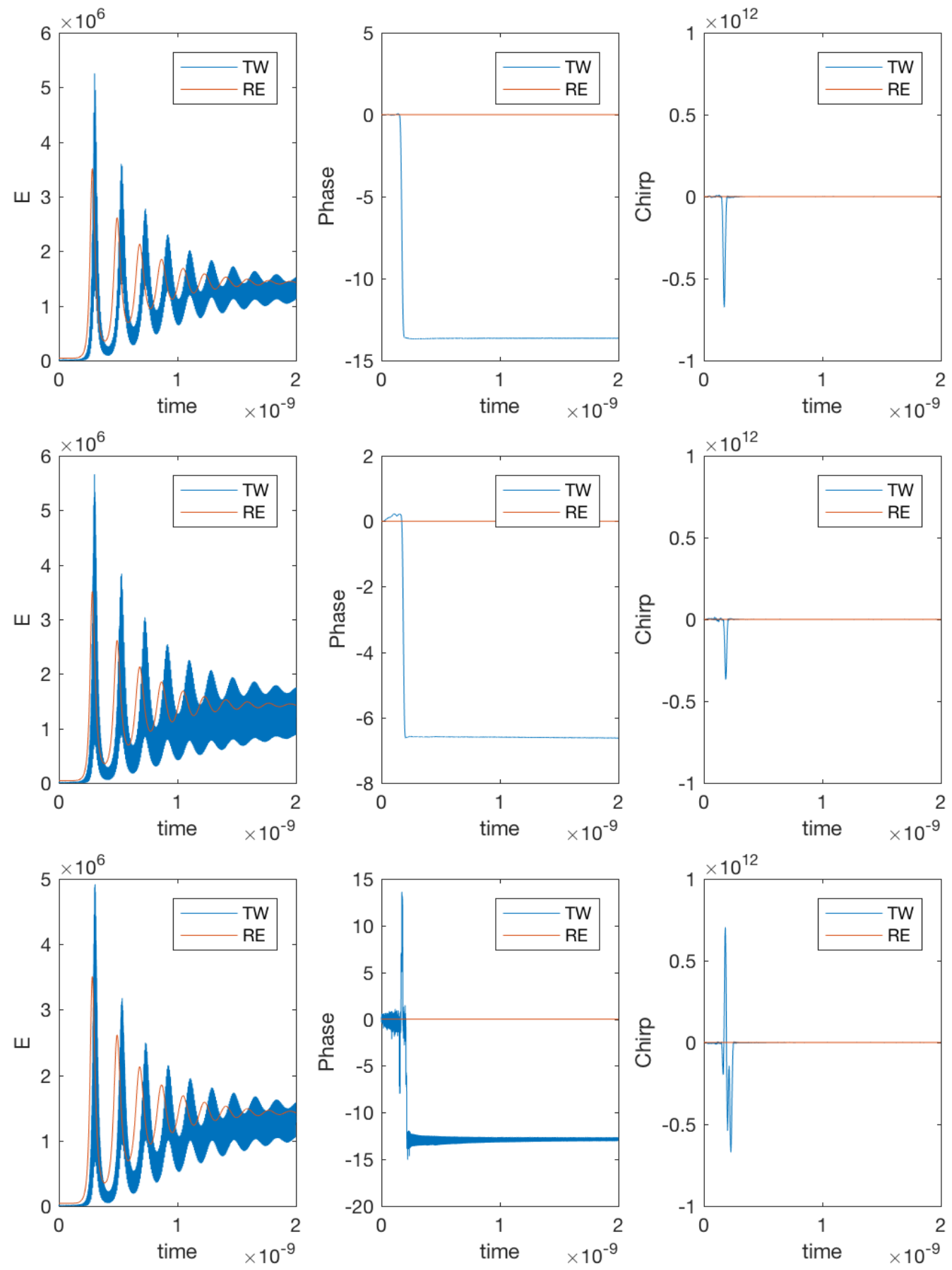

Figure 7.15: E-field, phase and chirp for three runs of single-mode REM compared with TWM. Henry factor set to 0 and $N$ starting at threshold. 

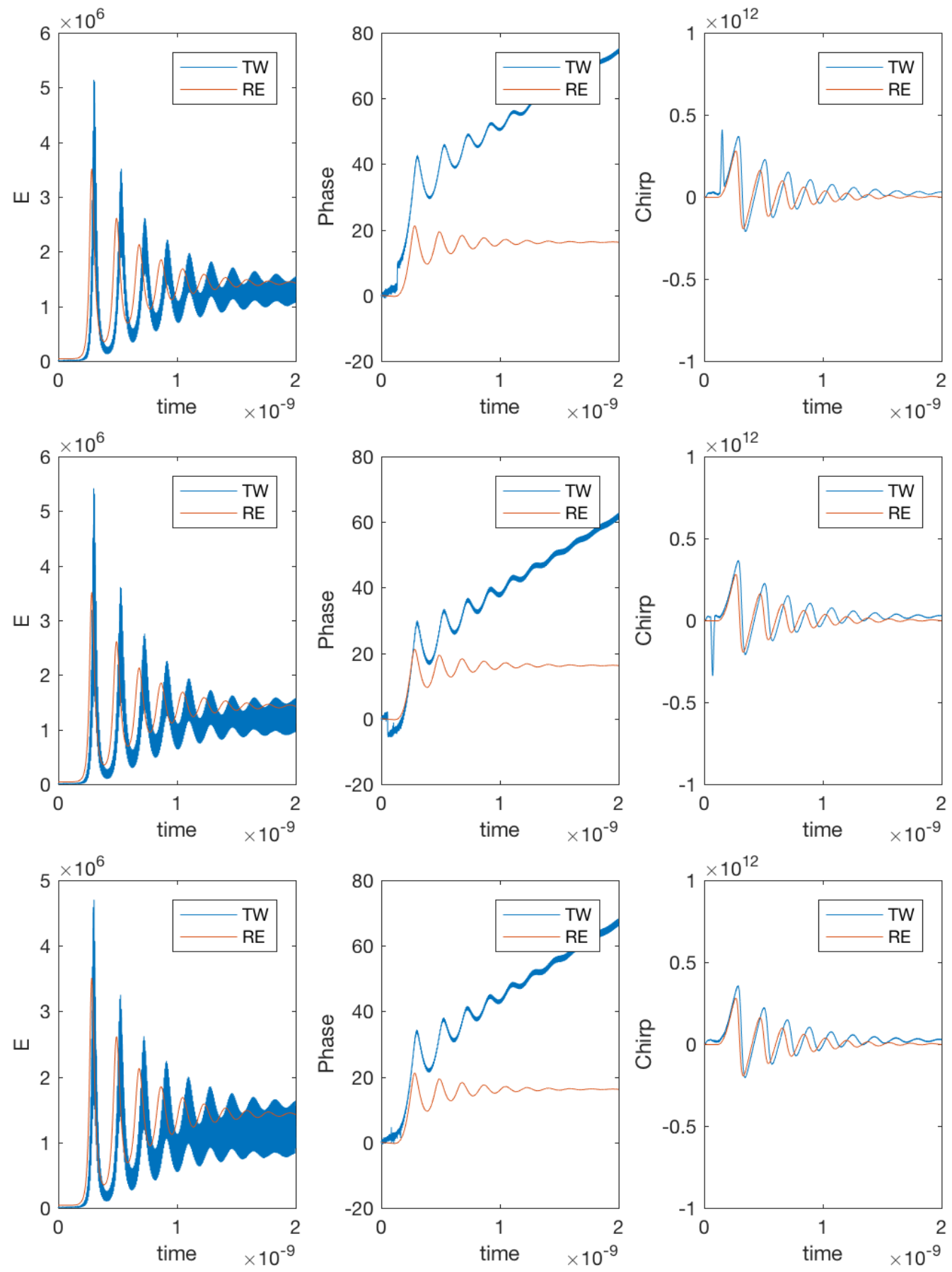

Figure 7.16: E-field, phase and chirp for single-mode run of REM and TWM with Henry factor of 5 starting at threshold. 

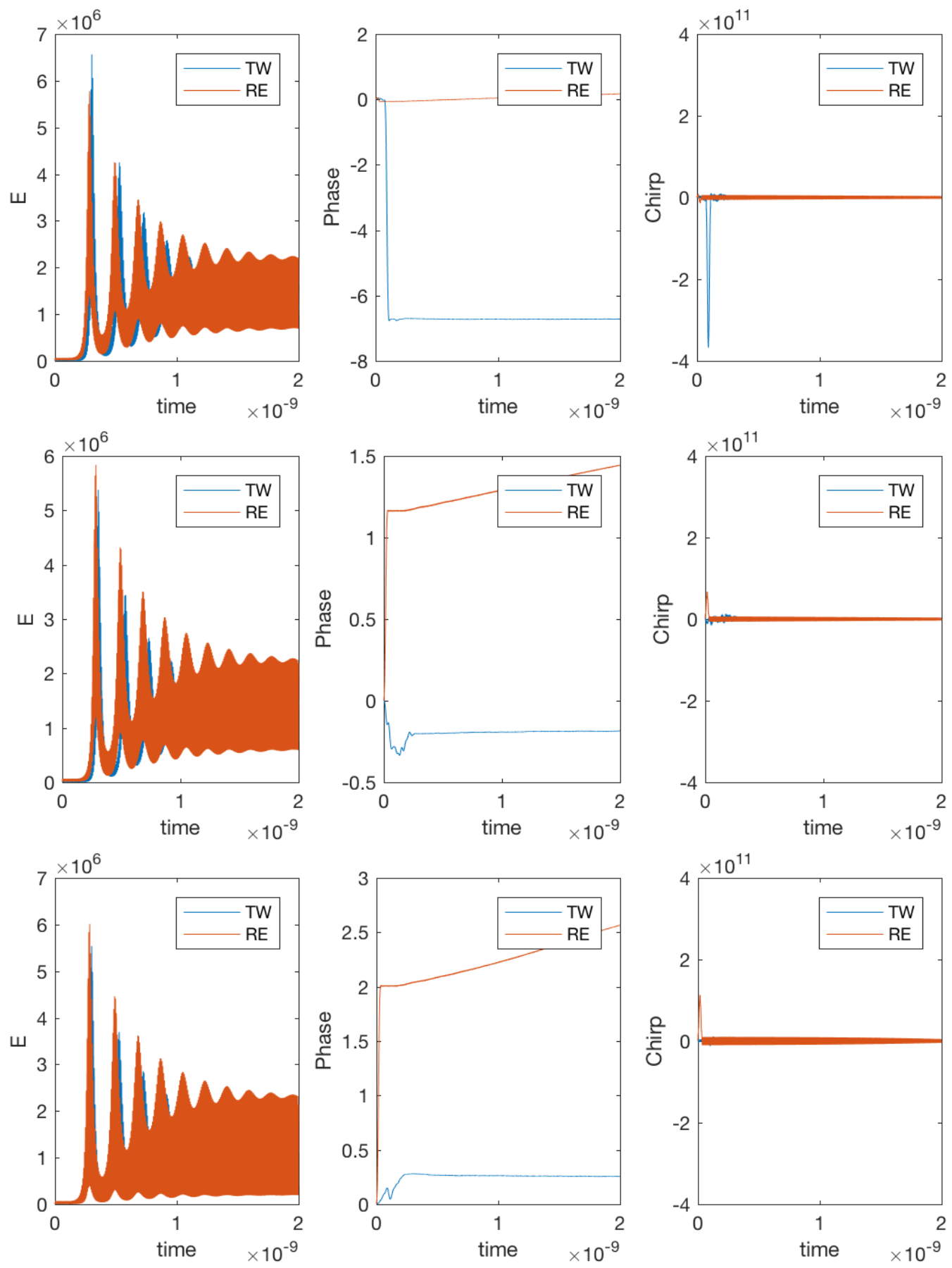

Figure 7.17: E-field, phase and chirp for three runs of 3-mode REM compared with TWM. Henry factor set to 0 and $N$ starting at threshold. Note that the phase had to be filtered. 

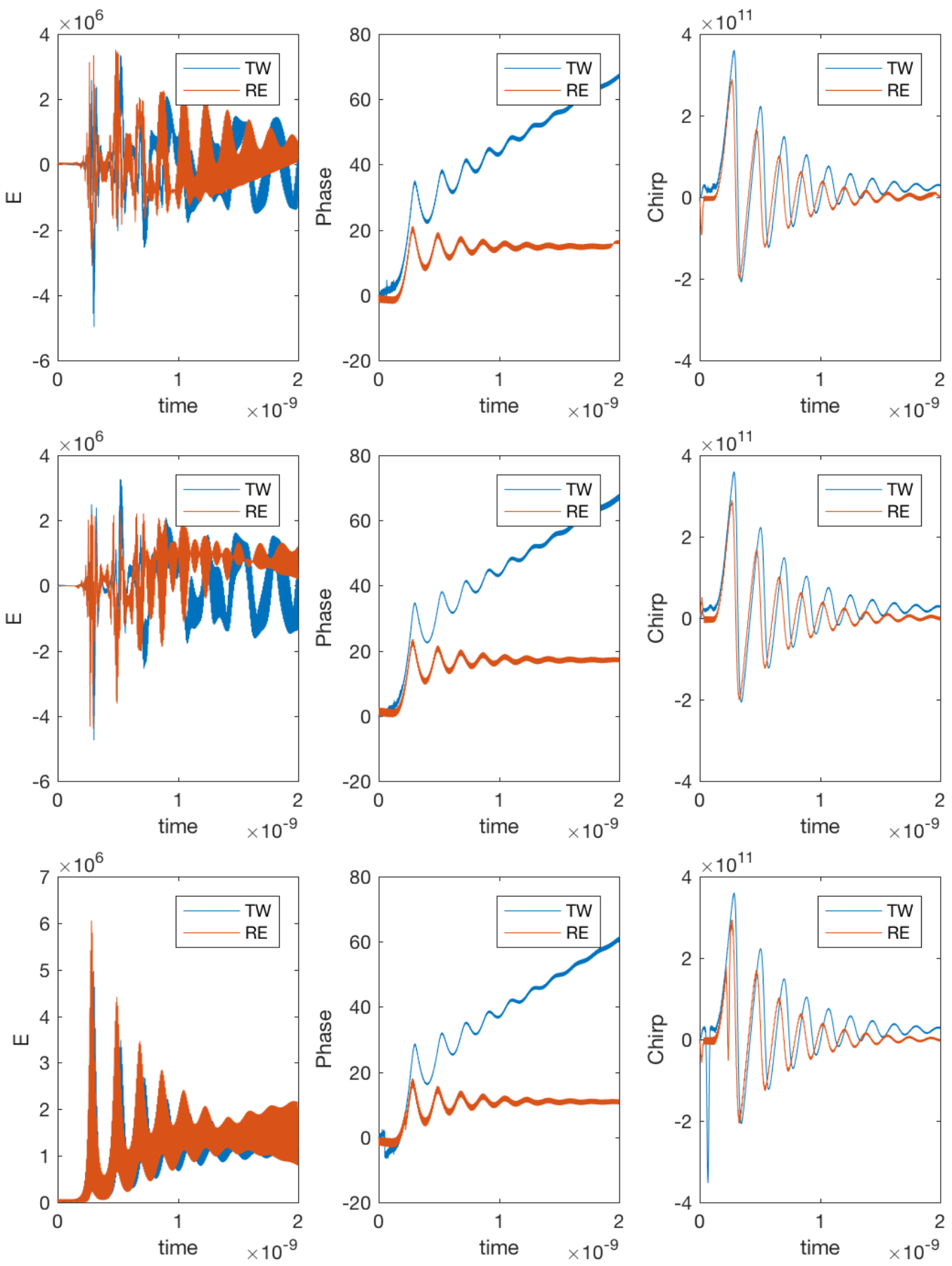

Figure 7.18: E-field, phase and chirp for three runs of 3-mode REM compared with TWM. Henry factor set to 5 and $N$ starting at threshold. 

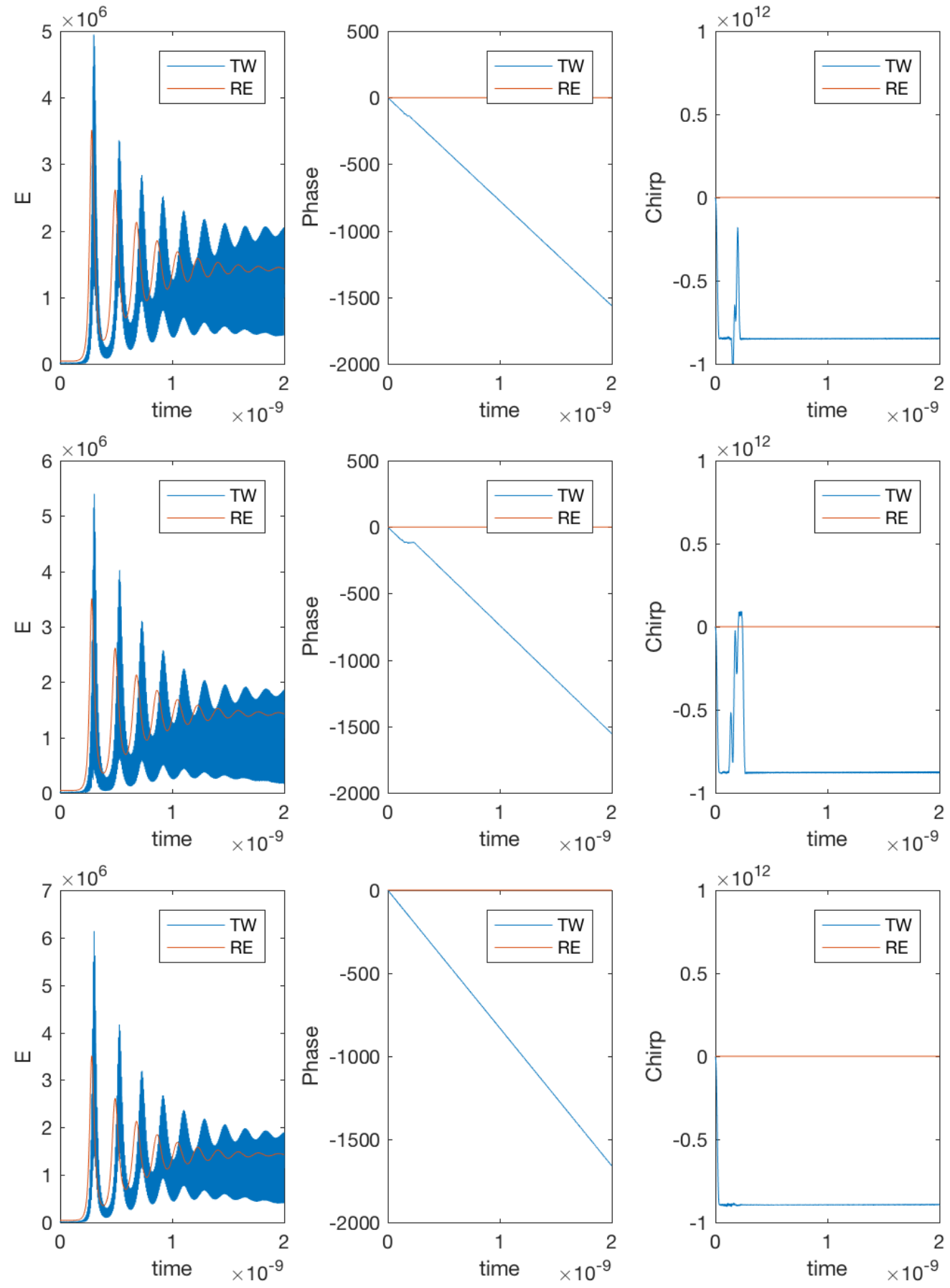

Figure 7.19: $E$-field, phase and chirp for three runs of single-mode REM compared with TWM. Henry factor set to 0 and $N$ starting at threshold. First run has $\delta=190$, 2nd $\delta=196.5, \operatorname{3rd} \delta=200$. 

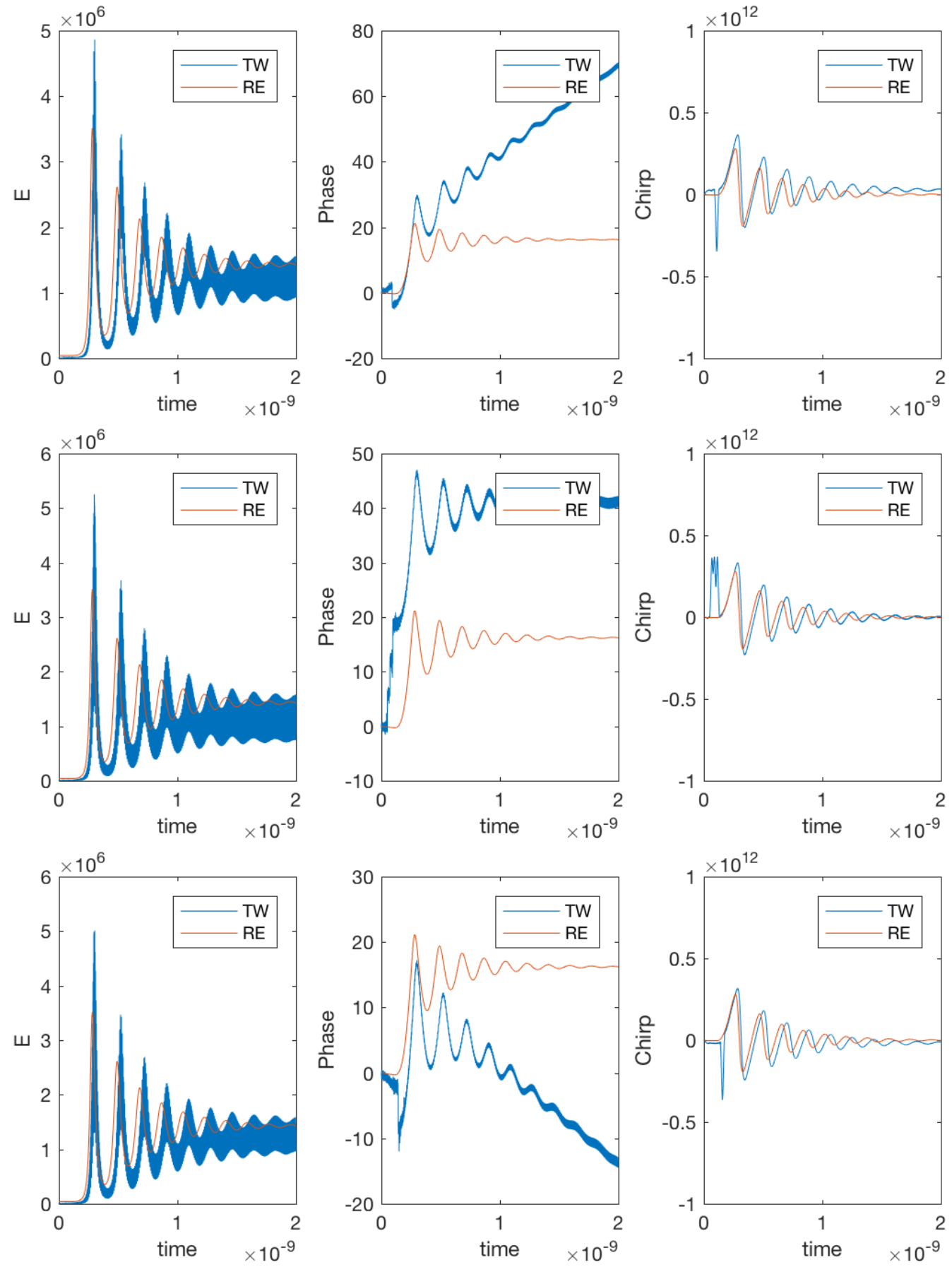

Figure 7.20: $E$-field, phase and chirp for three runs of single-mode REM compared with TWM. Henry factor set to 5 and $N$ starting at threshold. First run has $\delta=190$, 2nd $\delta=196.5,3$ rd $\delta=200$. Note: TWM not affected by REM modes. 


\subsection{Computational Cost}

We have already seen that the TWM incurs a significant hit every time step over the REM. Provided the TWM has increased accuracy the extra computational cost may be worth it. Alternatively, the detail given by the distributed nature of the model with respect to the physical structure of the model may also make it worthwhile to incur the extra cost. As stated earlier in section 3.6 the single-mode model is not appropriate for many lasers. In these cases the multi-mode REM is used. As more modes are included the cost goes up proportionately. At a point even the comparatively simple multi-mode REM implementation becomes costly. For our MNA engine a plot of compute time is shown in 7.21. As the number of modes increases the computational time goes up linearly. The TWM can be run faster at the sacrifice of bandwidth and accuracy. This is done by reducing the number of slices (segments); since the speed of the waves are approximately constant the number of segments sets the limit on the frequency that can be handled. For this comparison we cannot rely on the absolute numbers as this was implemented in Matlab. Converting to a compiled language like $\mathrm{C}$ will speed the simulation up considerably, but the relative times should be the same. For these numbers of modes and segments the $N$ and $S$ pulse response were all still reasonably accurate and the output plots remain the same as in figure 7.3. Even when the TWM has only 3 segments the output is not that far off.

It is important to realize that the number of segments (or discretization in the $z$-domain) for the TWM is not equivalent to the number of modes for the REM. It is not reasonable to use such a low segment number but the point is that for basic dynamics of the carrier and photon densities the computational time is comparable. A more realistic segment number for a laser would be 50 and typical multi-mode semiconductor lasers do not use more than 5 modes. This shows that for 'real' lasers the multi-mode REM will typically be faster than the TWM as expected.

If we take a look at the spectrums of the two simulations we find that at lower numbers the TWM bandwidth is about double that of the REM with the same number of modes. See figure 7.22. With 21 segments we get about the same bandwidth as a 41-mode REM. This shows that there is a limit where the TWM is actually more efficient. As the number of segments for the TWM increases the relationship is not as clear. In figure 7.23 the 51

segment TWM seems like it would be comparable to a 51-mode REM. The cost/performance comparison will depend on the particular laser being modeled. 


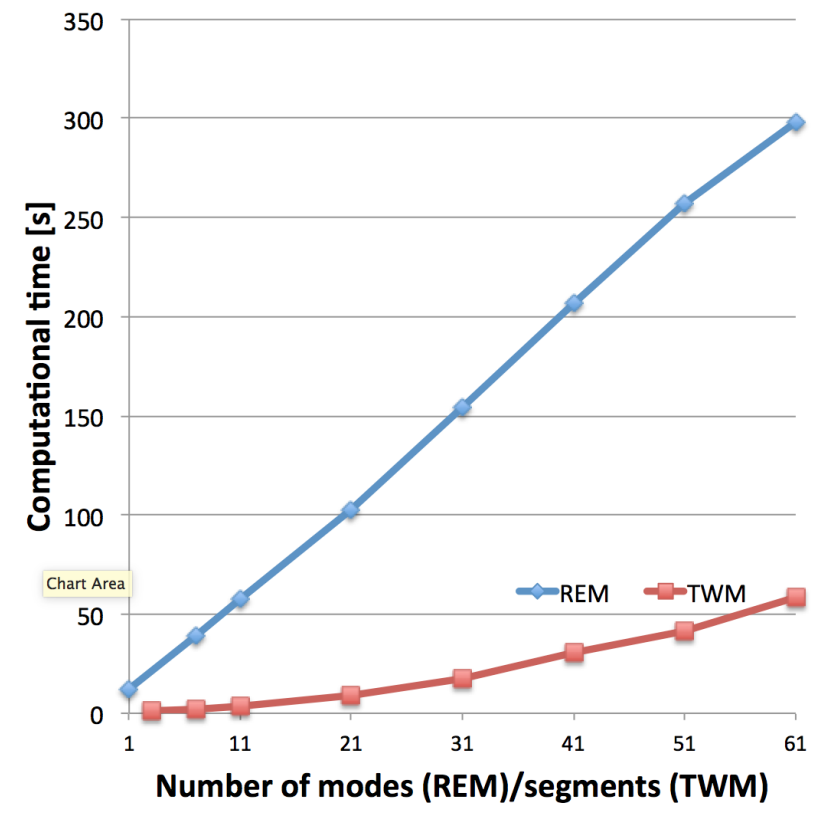

Figure 7.21: Computation time for TWM and various numbers of modes for the multi-mode REM.
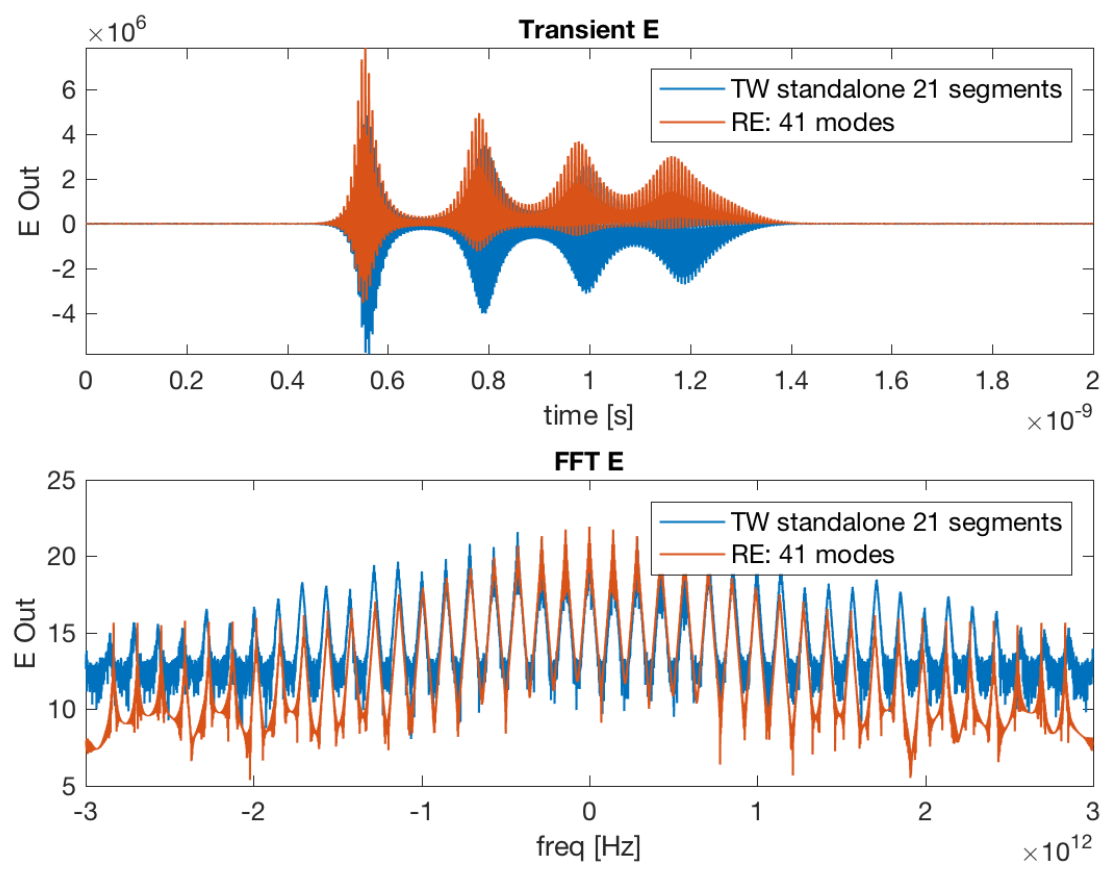

Figure 7.22: Comparing 41-mode REM and 21-segment TWM E-field and spectrum. The number of modes required to match the number of segments is about double. 

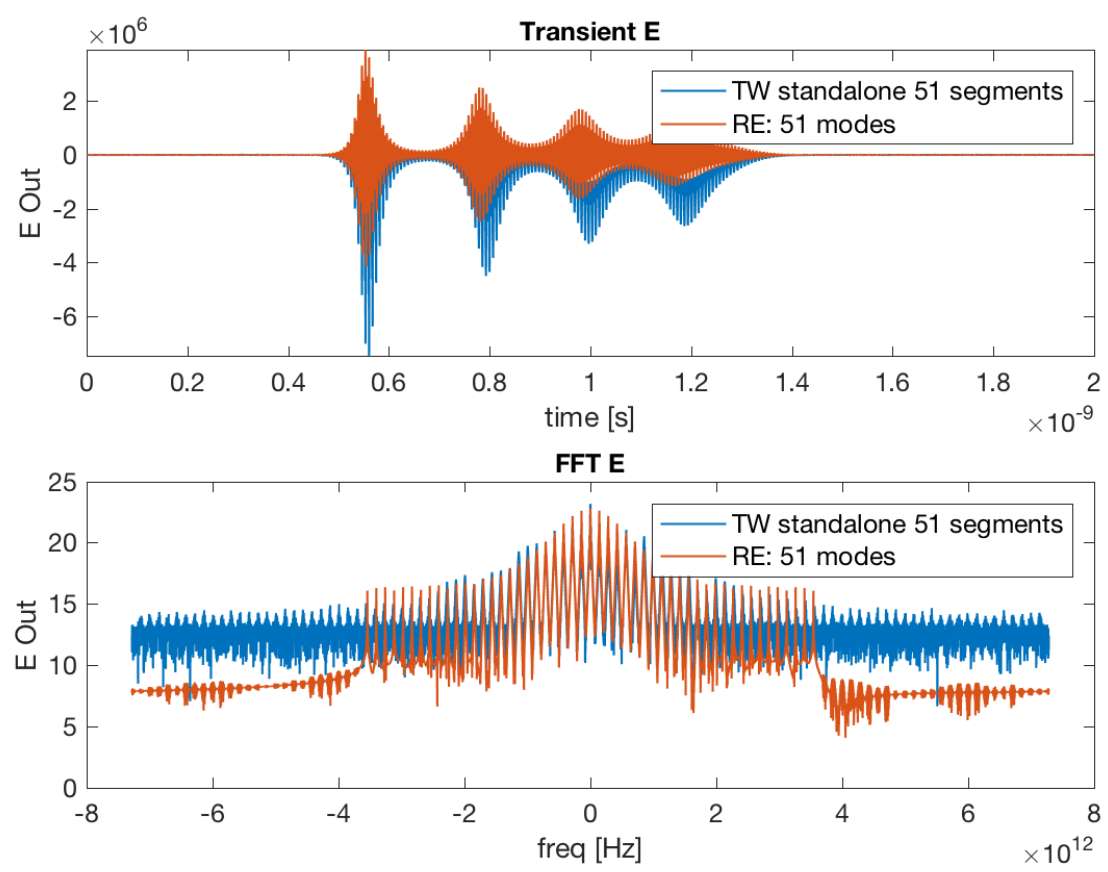

Figure 7.23: Comparing 51-mode REM and 51-segment TWM E-field and spectrum. The plot shows that the number of modes required to match the number of segments is about equal. 


\subsection{Summary}

Since the REM is an approximation to Maxwell's equations and the TWM is derived from Maxwell's equations we expect the models to have the same basic dynamics. This is indeed the case and as mentioned earlier deriving the REM from the TWM is explored in appendix I.

The distributed nature of the TWM is the main advantage of the model. By modeling the actual physical device it allows us to go directly from Maxwell's equations to traveling waves in a very intuitive way. And this also facilitates the specification of many parameters as they actually are in the physical device rather than averaging theses effects and creating a lumped model. The distributed fields make it easy to extend the model and add spatial features such as carrier diffusion and hole burning. It would also be easy to extend to DFB lasers. Although we have not shown the TWM to be more accurate than the REM the belief is that it must inherantly be more accurate and as we move to higher bandwidth applications or shorter timescales the models will really start to diverge. Imagine, for example, a pulse whose field was shorter than the length of the laser; the REM could not be expected to produce a meaningful result. Still, in each of the many areas of interest, comparison with physical devices is necessary to confirm how the models perform. 


\section{Chapter 8}

\section{Conclusions}

This thesis set out to examine the various laser models in use and investigate their integration into commercial circuit simulators. In particular the TWM is considered as the best choice for a laser model that provides better device detail dynamics and yet still has comparable computational cost once we need to switch to the multi-mode REM. Integration into commercial simulators is desirable because although optical models, including laser models, are commonly used, they are generally coded by the designer and used standalone - outside of the electronic simulations that are so commonplace with circuit design.

We have studied several laser models that have been implemented within our own commercial simulator platform. These models were assembled from available research and tested and compared on various criteria.

The TLM was previously implemented as a standalone simulator and rejected. The lack of a complex envelope and difficulty in integrating with the MNA engine made it necessary to find a workaround. This was not deemed feasable. The main thrust then was to look at the REM and the TWM. The REM is widely used and gives good overall laser dynamics, but does not allow the parameterization of a general laser model. It is incredibly simple and fast and this justifies its widespread use. The TWM is physically-based and therefore has the potential to model as much detail as required. But these come at a cost and are difficult to prove to be correct.

The REM, being a lumped model will be simple but will not accept specific physical parameters for customization. Even something as basic as cavity modes must be added manually. This involves calculating the mode frequencies, their relative level and how many to include. Once we require the multi-mode REM its computational cost becomes comparable the the TWM. The REM is implemented in DEs so fits neatly into the MNA commercial SPICE-like simulation engine. Chirp is handled by the Henry factor and is a fitted parameter. This is also the case for the TWM. Other enhancements such as gain saturation have also been added to the model. Once all of these extensions are made to the classic REM we still only have a simple laser model that gives the overall laser dynamics.

The TWM, being a distributed model will be more complex than the REM. But it is then very natural for it to accept physical parameters of a real laser and incorporate them 
without prior knowledge of their effects. The best example of this is how the frequencies of the modes are naturally determined by the model once the physical dimensions and index of refraction are set. Even the index of refraction no longer needs to be a single number but may also vary over the entire model. The downside to the TWM then is the computational cost. Due to the synchronization condition a finer time step is required and it won't be synchronous with the MNA engine. This means a separate simulation must be set up and tracked for each main simulator time step. This adds overhead and therefore computational cost. This has been resolved and is only an issue that will result in higher computational requirements. It is not trivial to compare the computational cost, but if the single-mode REM is not adequate and more modes are required the additional cost of the multi-mode REM makes the costs comparable. In fact if fewer segments can be used in the TWM it may actually be more efficient.

For general laser direct modulation current switching, the TWM dynamic performance compares very well. After more complex scenarios are compared it is difficult to know for certain which model performs better. In this case we would need to verify with an actual laser. For integration with OptiSPICE the REM is much simpler but because it needs to be extended to provide better accuracy it turns out that the extension of modes alone will fairly quickly become more costly than the TWM. With respect to the Henry factor the TWM makes use of it in a very similar fashion.

Lastly, the number of different physical configurations and system configurations favour the TWM. For example, many optical elements do not have a resonant cavity, and the TWM can model these as well as a full laser. This is a viable benefit.

There are many areas that still need to be investigated. Ideally more laser parameters would be implemented and tested. Despite the detail facilitated by the distributed physical model there are many other model parameters that need to be included. Still, the most important factor that needs to be examined is how this model correlates with real lasers. It is recommended that simple lasers or SOA be parameterized and compared with the TWM.

This study shows that although there are many details, especially regarding accuracy, that cannot be definitively ascertained, the TWM is a great candidate for integrating into a commercial SPICE-like simulator. 


\section{Bibliography}

[1] T. Quarles, A. Newton, D. Pederson, and A. Sangiovanni-Vincentelli, SPICE 3 Version 3F5 User's Manual, Dept. of EECE, Univ. of California, Berkeley.

[2] T. Smy and J. H. Rasmussen, "Obtaining an operating point solution of a traveling wave laser model," 2015.

[3] T. Smy and J. H.Rasmussen, "Integration of traveling wave optical device models into an mna based circuit simulator," 2015.

[4] M. N. O. Sadiku, Elements of Electromagnetics, 4th ed. Oxford University Press, 2007.

[5] S. L. Chuang, Physics of Photonic Devices, 2nd ed. John Wiley \& Sons, Inc., 2000.

[6] R. Tucker and I. P. Kaminow, "High-frequency characteristics of directly modulated ingaasp ridge waveguide and buried heterostructure lasers," Journal of Lightwave Technology, vol. LT-2, no. 2, pp. 385-393, 1984.

[7] L. L. Chang and L. Esaki, "Semiconductor quantum heterostructures," Physics Today, vol. 45, pp. 36-43, Oct. 1992.

[8] R. P. Feynman, The Feynman Lectures on Physics. Basic Books, 2010.

[9] A. Yariv and P. Yeh, Photonics: Optical Electronics in Modern Communications, 6th ed. Oxford University Press, Jan 2006.

[10] C. H. Henry, "Theory of the linewidth of semiconductor lasers," IEEE Journal of Quantum Electronics, vol. 18, no. 2, pp. 259-264, 1982.

[11] M. Nizette, T. Erneux, A. Gavrielides, and V. Kovanis, "Nonlinear dynamics induced by external optical injection in semiconductor lasers," Physica D: Nonlinear Phenomena, vol. 161, no. 3-4, pp. 220-236, Jan. 2002.

[12] S.-C. Chan, "Rate equations analysis of optically injected semiconductor lasers for tunable microwave generation," 2008 IEEE PhotonicsGlobal@Singapore, pp. 1-4, Dec. 2002. 
[13] J. C. Cartledge and R. C. Srinivasan, "Extraction of dfb laser rate equation parameters for system simulation purposes," J. on Lightwave Tech., vol. 15, no. 5, pp. 852-860, Mar. 1997.

[14] A. J. Lowery, "New dynamic semiconductor laser model based on the transmission-line modelling method," IEE Proceedings J (Optoelectronics), vol. 134, no. 5, pp. 281-289, 1987.

[15] R. Tucker, "High-speed modulation of semiconductor lasers," Electron Devices, IEEE Transactions on, vol. LT-3, no. 6, pp. 1180-1192, 1985. [Online]. Available: http://ieeexplore.ieee.org/xpls/abs_all.jsp?arnumber $=1485133$

[16] S. O. Kasap, Optoelectronics and Photonics Principles and Practices, 2nd ed. Pearson, Oct 2013.

[17] J. E. Bowers, "High speed semiconductor laser design and performance," Solid State Electronics, vol. 30, pp. 1-11, 1987.

[18] E. J. Flynn, "Nature of wavelength chirping in directly modulated semiconductor lasers," Electronics Letters, vol. 20, no. 25-26, p. 1038, 1999. [Online]. Available: http://scitation.aip.org/content/aip/journal/jap/85/4/10.1063/1.369499

[19] T. Koch and J. Bowers, "A note on the semiconductor laser equivalent circuit," Journal of Applied Physics, vol. 85, no. 4, p. 2041, 1984. [Online]. Available: http://digital-library.theiet.org/content/journals/10.1049/el_19840709

[20] J. Carroll, J. Whiteaway, and D. Plumb, Distributed Feedback Semiconductor Lasers, 1st ed. Michael Faraday House, Six Hills Way, Stevenage, Herts. SG1 2AY, United Kingdom: The Institute of Electrical Engineers, 1998.

[21] C. Serrat, S. Prins, and R. Vilaseca, "Dynamics and coherence of a multimode semiconductor laser with optical feedback in an intermediate-length external-cavity regime," Physical Review A, vol. 68, no. 5, 2003.

[22] C.-W. Ho, A. Ruehli, and P. Brennan, "The modified nodal approach to network analysis," IEEE Transactions onCircuits and Systems, vol. 22, no. 6, pp. 504 - 509, June 1975.

[23] U. Wali, R. Pal, and B. Chatterjee, "On the modified nodal approach to network analysis," Proceedings of the IEEE, vol. 73, no. 3, pp. 485-487, March 1985.

[24] M. Nakhla, "Computer methods for analysis and design of vlsi and communication: Course notes 2013," 2013. 
[25] R. Čiegis and M. Radziunas, "Effective numerical integration of traveling wave model for edge-emitting broad-area semiconductor lasers and amplifiers," Mathematical Modelling and Analysis, vol. 15, no. 4, pp. 409-430, Jan. 2010. [Online]. Available: http://www.tandfonline.com/doi/abs/10.3846/1392-6292.2010.15.409-430

[26] J. Sieber, U. Bandelow, H. Wenzel, M. Wolfrum, and H.-j. Wünsche, "Travelling Wave Equations for Semiconductor Lasers with Gain Dispersion," no. 459, 1998. [Online]. Available: http://citeseerx.ist.psu.edu/viewdoc/summary?doi=10.1.1.54.7198

[27] J. Sieber, "Numerical Bifurcation Analysis for Multisection Semiconductor Lasers," SIAM Journal on Applied Dynamical Systems, vol. 1, no. 2, pp. 248-270, Jan. 2002. [Online]. Available: http://epubs.siam.org/ doiSieber,J.(2002).NumericalBifurcationAnalysisforMultisectionSemiconductorLasers. SIAMJournalonAppliedDynamicalSystems, 1(2),248?270.doi:10.1137/ S1111111102401746/abs/10.1137/S1111111102401746

[28] R. Čiegis, M. Radziunas, and M. Lichtner, "Numerical algorithms for simulation of multisection lasers by using traveling wave model," Mathematical Modelling and Analysis, vol. 13, no. 3, pp. 327-348, Jan. 2008. [Online]. Available: http://www.tandfonline.com/doi/abs/10.3846/1392-6292.2008.13.327-348

[29] W. Li, W.-p. Huang, and X. Li, "Digital filter approach for simulation of a complex integrated laser diode based on the traveling-wave model," IEEE Journal of Quantum Electronics, vol. 40, no. 5, pp. 473-480, 2004.

[30] H.-j. Wünsche and M. Radziunas, "Traveling-wave modeling of semiconductor lasers and amplifiers," pp. 1-54. [Online]. Available: http://www.nusod.org/nusod05/MB1.pdf

[31] P. Berini, "Numerical methods in electromagnetic engineering: Course notes 2013," 2013.

[32] C. Hirsch, Numerical Computation of Internal and External Flows, 2nd ed. Butterworth-Heinemann, 2007.

[33] U. Bendelow, M. Radziunas, J. Sieber, and M. Wolfrum, "Impact of gain dispersion on the spatio-temporal dynamics of multisection lasers," Quantum Electronics, IEEE Journal of, vol. 37, no. 2, pp. 183-188, Feb 2001.

[34] M. Radziunas, K.-H. Hasler, B. Sumpf, T. Q. Tien, and H. Wenzel, "Mode transitions in distributed bragg reflector semiconductor lasers: experiments, simulations and analysis," Journal of Physics B: Atomic, Molecular and Optical Physics, vol. 44, no. 10, pp. 327-348, May 2011. [Online]. Available: http://stacks.iop.org/0953-4075/ $44 / \mathrm{i}=10 / \mathrm{a}=105401$ ?key $=$ crossref.4b4fc2f6a93a229c7023f3cf73254c0c 
[35] M. Rossetti, P. Bardella, and I. Montrosset, "Time-domain travelling-wave model for quantum dot passively mode-locked lasers," IEEE Journal of Quantum Electronics, vol. 47, no. 2, pp. 139-150, feb 2011.

[36] T. B. Simpson, J. M. Liu, K. F. Huang, and K. Tai, "Nonlinear dynamics induced by external optical injection in semiconductor lasers," Quantum and Semiclassical Optics: Journal of the European Optical Society Part B, vol. 9, no. 5, pp. 765-784, Oct. 1997.

[37] J. Javaloyes and S. Balle, "Emission directionality of semiconductor ring lasers: A traveling-wave description," IEEE Journal of Quantum Electronics, vol. 45, no. 5, pp. 431-438, 2009. [Online]. Available: http://www.tandfonline.com/doi/abs/10.3846/ 1392-6292.2008.13.327-348

[38] G. R. Luevano., "Optical/electrothermal simulation of integrated ridge lasers," 2001.

[39] L. D. Landau and E. M. Lifshitz, Electrodynamics of Continuous Media, 1st ed. Pergamon Press, 1960.

[40] J. E. Carroll, Distributed Feedback Semiconductor Lasers, 1st ed. Cambridge: Cambridge University Press, 1985. 
Appendix A

\section{Interference and Delay through a waveguide}




\section{A.1 Calculating Phase}

Usually phase is calculated with respect to a reference. For example if we have two carriers we can calculate the phase of one with respect to the other and would then be able to determine how the two waves interfere with each other. In this case

$$
\begin{gathered}
E_{r e f}(z, t)=e^{-i \omega_{c} t} \\
E_{1}(z, t)=e^{i\left(k_{c} z_{1}-\omega_{c} t\right)}
\end{gathered}
$$

and the relative phase is equal to

$$
\phi(z, t)=\tan ^{-1}\left\{\frac{e^{i\left(k_{c} z_{1}-\omega_{c} t\right)}}{e^{-i \omega_{c} t}}\right\}=\tan ^{-1}\left\{e^{i k_{c} z_{1}}\right\}=k_{c} z_{1}
$$

The relative phase calculation is trivial but this shows that the carrier phase, although hidden, will have to be taken into account when signals are propagated in space.

\section{A.2 Example: Phase Change Due to Full-wavelength Carrier Delay}

Consider the propagation of a wave through a waveguide of length $L$; specifically define a plane wave traveling right. The field equation including the carrier is then

$$
E(z, t)=A(z, t) e^{i\left(\omega_{c} t-k_{c} z\right)}
$$

The delay through the waveguide is $t_{L}$ where $v$ is the phase velocity and $L=v t_{L}$. This is true for both the envelope $A(z, t)$ and the carrier. Note this assumes that the phase velocity is equal to the group velocity which is shown in section G.1. An example of a carrier modulated by a square pulse is shown in figure A.1. The top plot shows the wave over distance. The waveguide is demarcated by $z_{0}$ and $z_{1}$ and the waveguide is exactly one wavelength long. The plot shows the sqare wave pulse after it has emerged from the waveguide. (The square wave is drastically shortened for illustration purposes and does not meet the SVEA requirement.)

The two bottom plots show the wave versus time at both ends of the waveguide. We can see that the pulse emerges from the right side $t_{L}$ after it enters the left side and that the model represents the phase relationship of the input and output. We can see that in this case the phase change of the carrier is $2 \pi$ so the output of the waveguide has the correct phase including the delay in the carrier. So if the delay $t_{L}$ is a multiple of $T_{c}$ (or $L$ is a multiple of $\left.\lambda_{c}\right)$, then the delay of the carrier will have no affect on the envelope phase. If, however, $t_{L}$ is not a multiple of $T_{c}$, the delay of the carrier will have to be taken into account. 

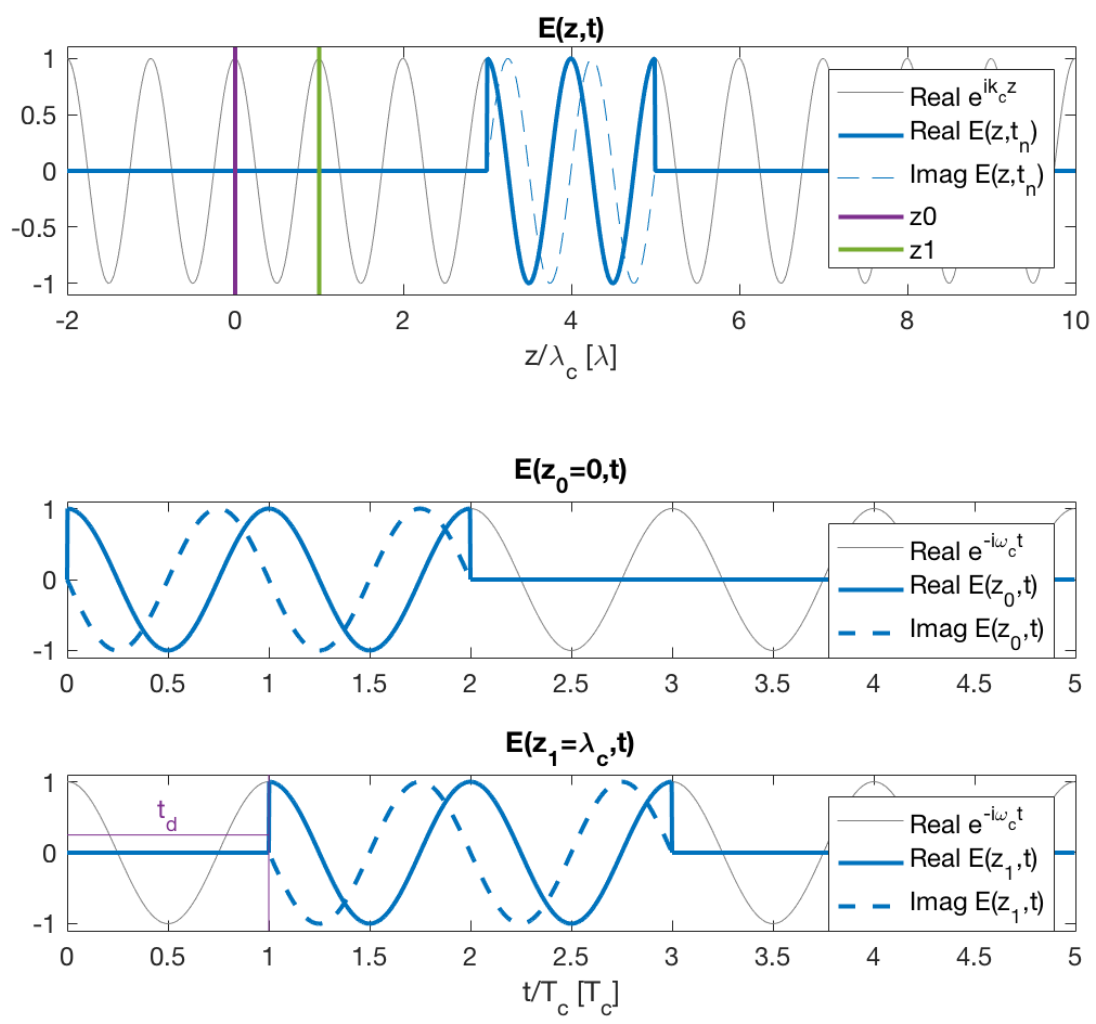

Figure A.1: Delay of carrier modulated with a square pulse through waveguide without gain or loss. Top shows physical extent. Bottom 2 show signals versus time. 2nd shows input at left side $(z=0)$. Bottom shows output after a delay of $\lambda\left(z=z_{1}\right)$.

\section{A.3 Example: Phase Change Due to Half-wavelength Carrier Delay}

Consider figure A.2. In this example the waveguide is half a wavelength (shown in the top plot by the $z_{0}$ and $z_{1}$ lines) while the original one-wavelength waveguide is now demarcated by end points $z_{0}$ and $z_{2}$. If we look at the output of the half-wavelength waveguide $\left(E\left(z_{1}, t\right)\right)$, we see that the square wave has been shifted properly by the model but that the carrier is now half a cycle out of phase with the input. It is clear that adding the envelope of the input and output would give 2 where they overlap which in this case is incorrect as the carriers would destructively interfere actually giving 0 . This is why any system level simulations must account for carrier delay explicitly. For this reason, if we are free to choose $\omega_{c}$ we will always choose the first mode of the waveguide (whose half- $\lambda$ is an integral number of the length $L$ ). If this is the case the phase of the carrier does not change from input to output and we can add the signals without explicitly managing the carrier phase change. 

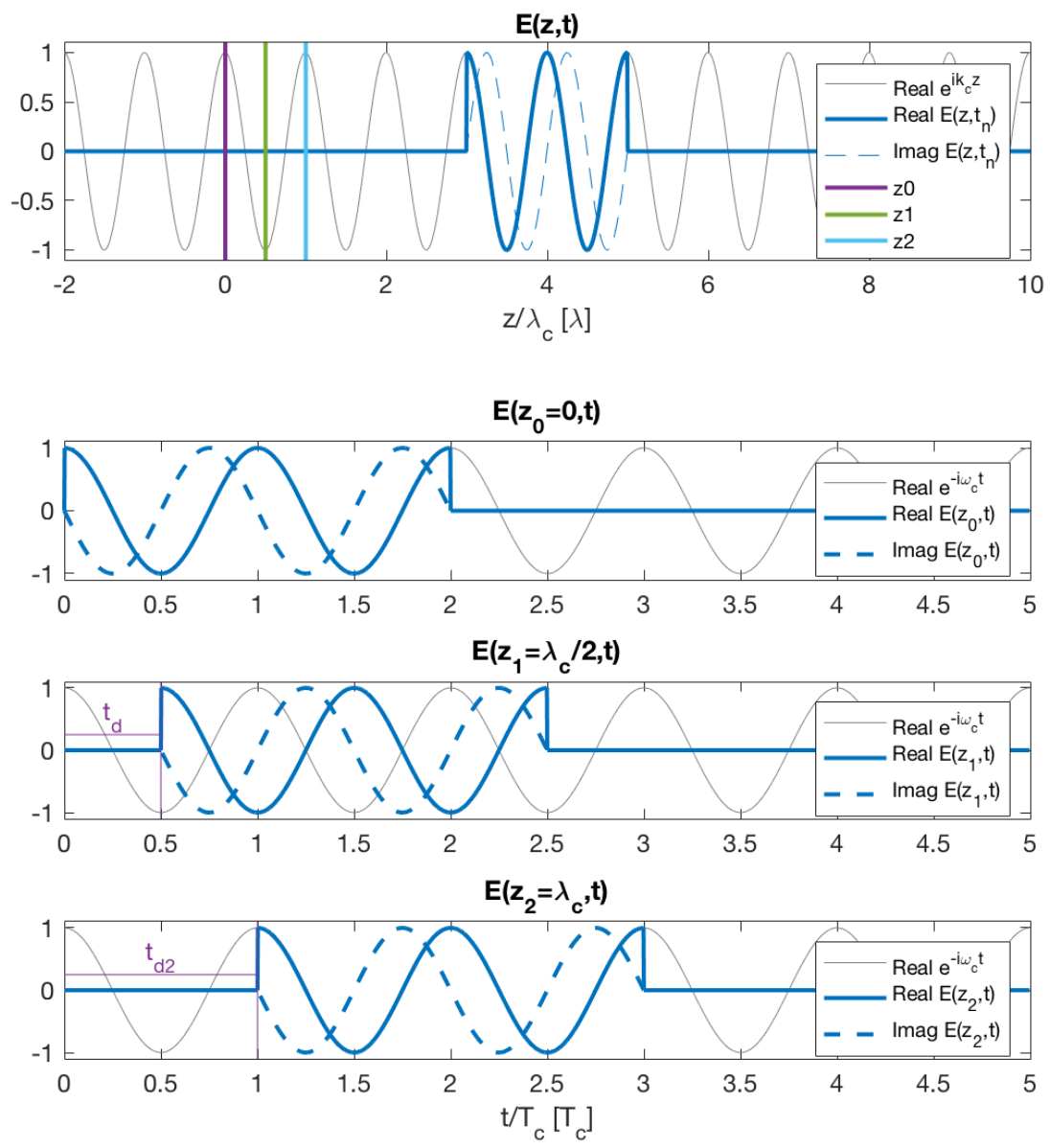

Figure A.2: Delay of carrier modulated with a square pulse through waveguide without gain or loss. Top shows physical extent. Bottom 3 show signals in time. 2nd shows input at left side $(z=0)$. 3rd shows input after a delay of $\frac{\lambda}{2}\left(z=z_{1}\right)$. Bottom shows output at right side after a delay of $\lambda\left(z=z_{2}\right)$. 


\section{A.4 Phase change due to arbitrary carrier delay}

When we speak of the phase shift of the model, it can mean different things. In this case we are speaking of the phase shift with respect to the carrier. This will allow us to handle interference between signals at different parts of a system. Given a reference input to a waveguide at $z=0$ (reference is a traveling wave measured at $z=0$ )

$$
E_{r e f}(z, t)=E(0, t)=e^{-i \omega_{c} t}
$$

At the waveguide output $z_{1}$ we have

$$
E\left(z_{1}, t\right)=A\left(z_{1}, t\right) e^{i\left(k_{c} z_{1}-\omega_{c} t\right)}
$$

The difference in the carrier phase is $\triangle \phi=k_{c} z_{1}$

Note that this means that even though the envelope phase relative to its carrier stays fixed - both waves travel at same speed and so are always aligned the same - the envelope phase relative to the reference carrier (at $z=0$ ) will change as the waves move through the waveguide. This is clear if one looks at figure A.1.

If we start with the output of the waveguide

$$
E(L, t)=A(L, t) e^{i\left(k_{c} L-\omega_{c} t\right)}
$$

we can substitute for the envelope $E(L, t)=E\left(0, t-t_{L}\right)$ to yield

$$
E(L, t)=A\left(0, t-t_{L}\right) e^{i\left(k_{c} L-\omega_{c} t\right)}
$$

which we can rewrite as

$$
\begin{gathered}
E(L, t)=A\left(0, t-t_{L}\right) e^{i k_{c} L} e^{-i \omega_{c} t} \\
E(L, t)=A_{o}(L, t) e^{-i \omega_{c} t}
\end{gathered}
$$

where $A_{o}$ is the new envelope at the output, referenced to the same carrier reference

$$
A_{o}(L, t)=A\left(0, t-t_{L}\right) e^{i k_{c} L}
$$

This is just the shifted envelope with a phase factor from the delayed carrier. In the model then, whenever the phase relative to the carrier is relevent, we will have to add this phase factor to our output signal. This can be done by a static detuning factor. See section 5.9. 


\section{A.5 Phase change due to different carrier frequencies}

When two complex envelopes with different carrier frequencies need to be combined, this cannot necessarily be done directly. Although not as simple as a constant phase difference it also results in including a phase factor in the envelope; one of the carriers is chosen to be the reference, the other will require the phase factor. Figure A.3 shows two carriers close in frequency with constant envelope amplitudes of 1 . For simplicity let's align them at $z=0$.

$$
\begin{gathered}
E_{\text {ref }}(0, t)=e^{-i \omega_{c} t} \\
\begin{aligned}
E_{1}(0, t) & =e^{-i \omega_{1} t} \\
& =e^{-i\left(\omega_{1}-\omega_{c}\right) t} e^{-i \omega_{c} t} \\
& =e^{-i \delta t} e^{-i \omega_{c} t} \\
E_{1} & =e^{-i \delta t} e^{-i \omega_{c} t}
\end{aligned}
\end{gathered}
$$

where $\delta=\omega_{c}-\omega_{1}$. The figure shows that this does indeed match the original $E_{1}$.

You can also see how in the complex envelope representation the carrier or reference becomes the constant $e^{i 0}=1+i 0$ and $E_{1}=e^{-i \delta t}$. On a phase plot $E_{1}$ is a straight line of linearly increasing phase. 

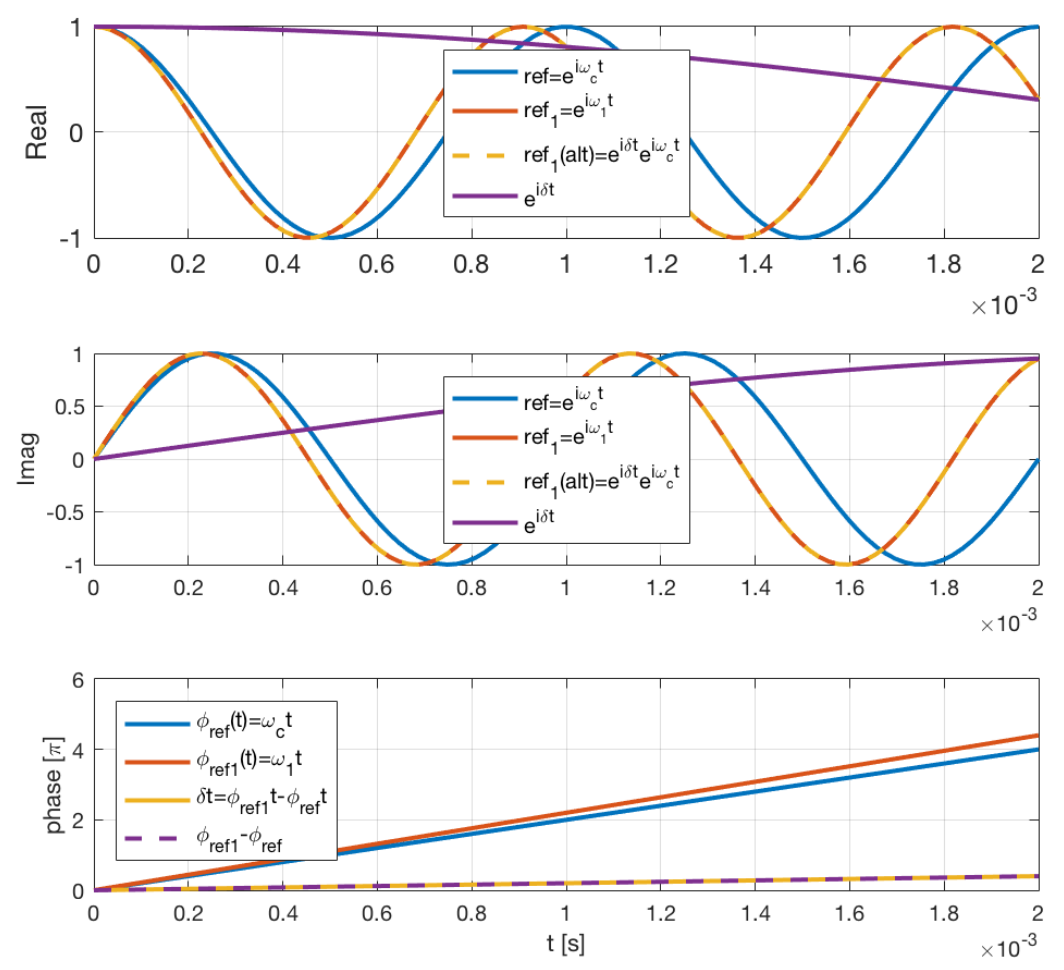

Figure A.3: Phase factor due to difference in carrier frequencies.

\section{A.6 Interference and Mode Generation in a Resonant Cavity}

It would seem straightforward that if we had a traveling wave propagating in a resonant cavity that the constructive and destructive interference would result in only modes of the cavity being present. This is not the case. If the boundary conditions at the ends of the cavity were forced to zero then, like a string with its ends bound, the modes of the cavity would appear. But in the traveling wave resonant cavity the ends are mirrors and not forced to zero. In fact any wave present in the TWM at startup will just circulate around and around the cavity. Not even the Lorentzian filtering can help as all it will do is reduce any frequencies already present. How then, do the appropriate modes appear?

The answer lies in the physical nature of the model and how we must model the situation. In a real system we would not expect any frequencies present unless there is some way they are being added. This is what happens in our model but of course we must model this. The other frequencies are added via spontaneous emission. With random signals added to our cavity we will have energy at all frequencies.

So if the interference isn't forced by the endpoints of the cavity how does it occur? Let's 
say we take a signal that is a perfect sine wave but not of integral wavelengths. This means that the spectrum contains some high frequency components from the discontinuity at the point where one end of the sine wave meets the other. These high frequency components will be filtered and the sine wave left. 


\section{Appendix B}

\section{Counter Rotating Waves}

Consider the TWM simple equation for forward traveling wave (5.3)

$$
\frac{\partial E}{\partial t}+v_{g} \frac{\partial E}{\partial z}=0
$$

For the counter clockwise rotating exponential

$$
E=A e^{i\left(k_{e} z-\omega_{e} t\right)}
$$

we have from (5.5)

$$
v_{g}=\frac{\omega_{e}}{k_{e}}
$$

and the same for the clockwise rotating exponential

$$
E=A e^{-i\left(k_{e} z-\omega_{e} t\right)}
$$

For the TWM real $\beta$ equation for forward traveling wave (5.8)

$$
\frac{\partial E}{\partial t}+v_{g} \frac{\partial E}{\partial z}=-i v_{g} \beta E
$$

and the $\mathrm{CCW}$ rotating exponential

$$
E=A e^{i\left(k_{\beta} z-\omega_{e} t\right)}
$$

from (5.11) we get

$$
k_{\beta}=k_{e}-\beta
$$

But for the $\mathrm{CW}$ rotating exponential

$$
E=A e^{-i\left(k_{\beta}^{\prime} z-\omega_{e} t\right)}
$$


we get

$$
\begin{aligned}
& i \omega_{e} E-v_{g} i k_{\beta}^{\prime} E=-i v_{g} \beta E \\
& \omega_{e}-v_{g} k_{\beta}^{\prime}=-v_{g} \beta \\
& \frac{\omega_{e}}{v_{g}}-k_{\beta}^{\prime}=-\beta \\
& k_{\beta}^{\prime}=k_{e}+\beta
\end{aligned}
$$

When we sum the two counter rotating waves

$$
\begin{aligned}
E & =A\left[e^{i\left(k_{\beta} z-\omega_{e} t\right)}+e^{-i\left(k_{\beta}^{\prime} z-\omega_{e} t\right)}\right] \\
& =A\left[e^{i\left(\left[k_{e}-\beta\right] z-\omega_{e} t\right)}+e^{-i\left(\left[k_{e}+\beta\right] z+\omega_{e} t\right)}\right] \\
& =A\left[e^{-i \beta z} e^{i\left(k_{e} z-\omega_{e} t\right)}+e^{-i \beta z} e^{-i\left(k_{e} z-\omega_{e} t\right)}\right] \\
& =A e^{-i \beta z}\left[e^{i\left(k_{e} z-\omega_{e} t\right)}+e^{-i\left(k_{e} z-\omega_{e} t\right)}\right] \\
& =2 A e^{-i \beta z} \cos \left(k_{e} z-\omega_{e} t\right)
\end{aligned}
$$

we get a rotating real cosine traveling at speed $v_{g}$. Even though the CCW wave slows down and the CW wave speeds up when we add them the resulting wave travels at the original speed of the individual waves. Plots for the waves are given in figures B.1, B.2 and B.3. 

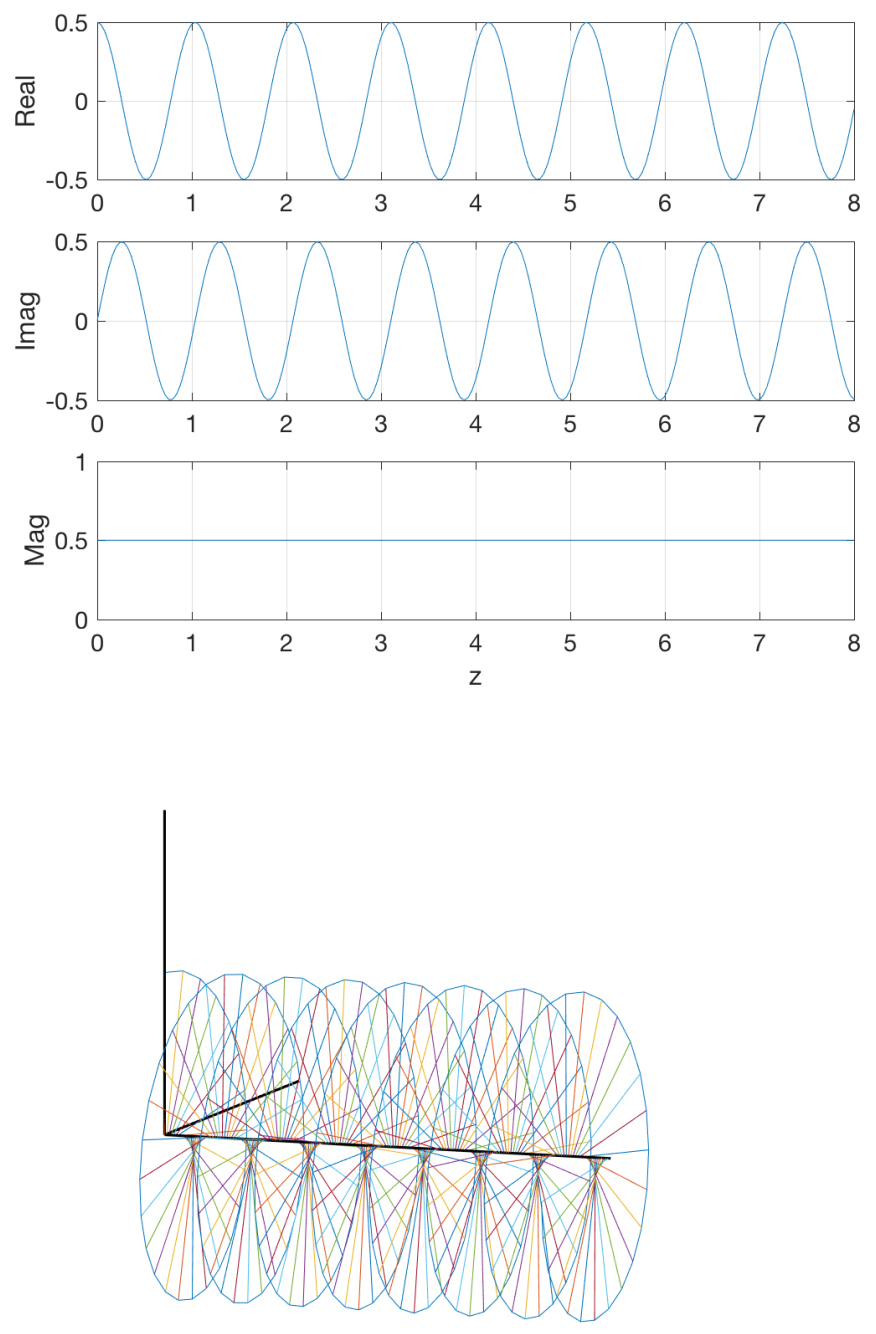

Figure B.1: Complex exponential $e^{-i \beta z} e^{i\left(k_{e} z-\omega_{e} t\right)}$ rotating CW. Bottom 3-D plot shows complex value over $z$ : Left/right axis is $z$, other two are real/imaginary. 

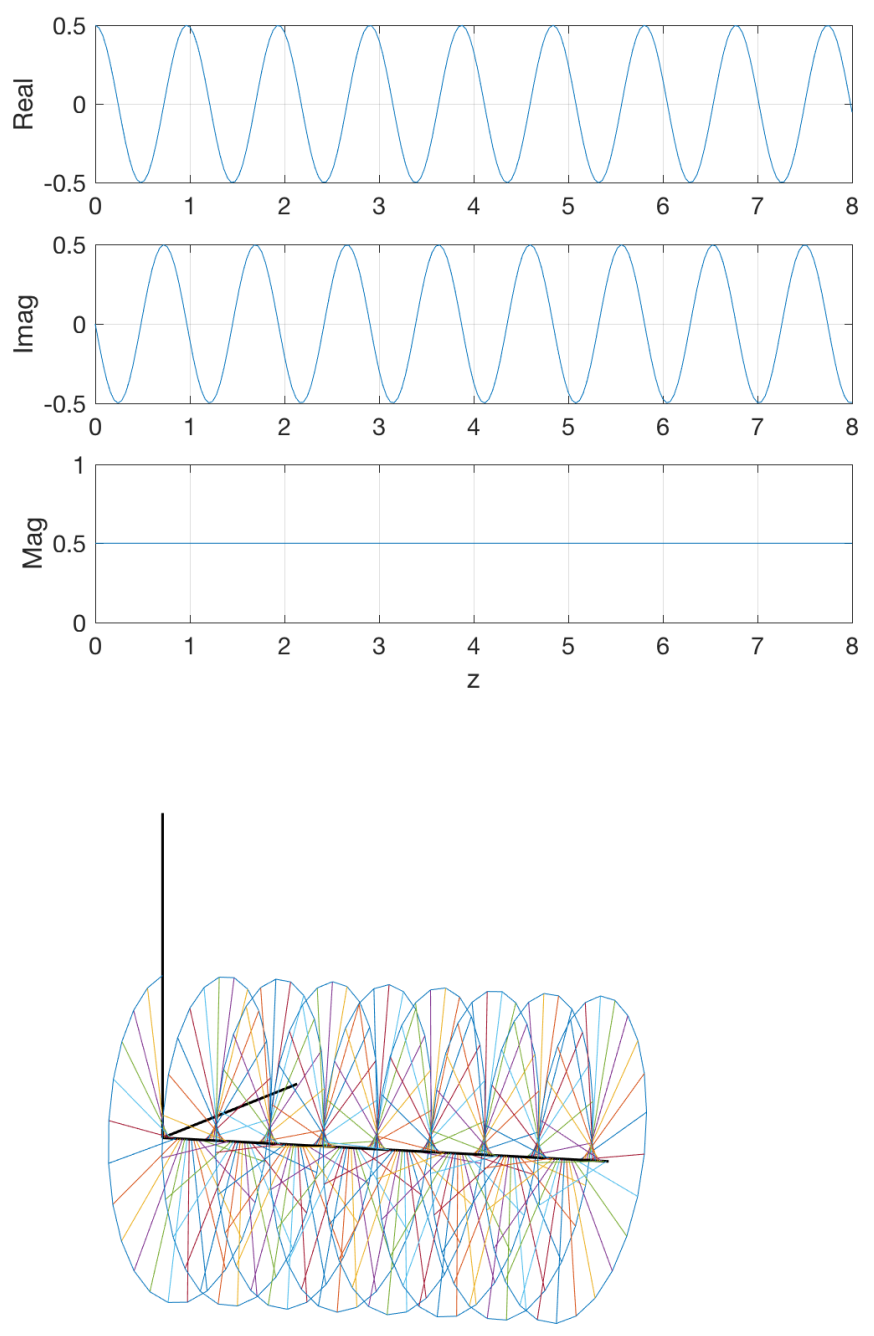

Figure B.2: Complex exponential $e^{-i \beta z} e^{-i\left(k_{e} z-\omega_{e} t\right)}$ rotating CCW. Bottom 3-D plot shows complex value over $z$ : Left/right axis is $z$, other two are real/imaginary. 

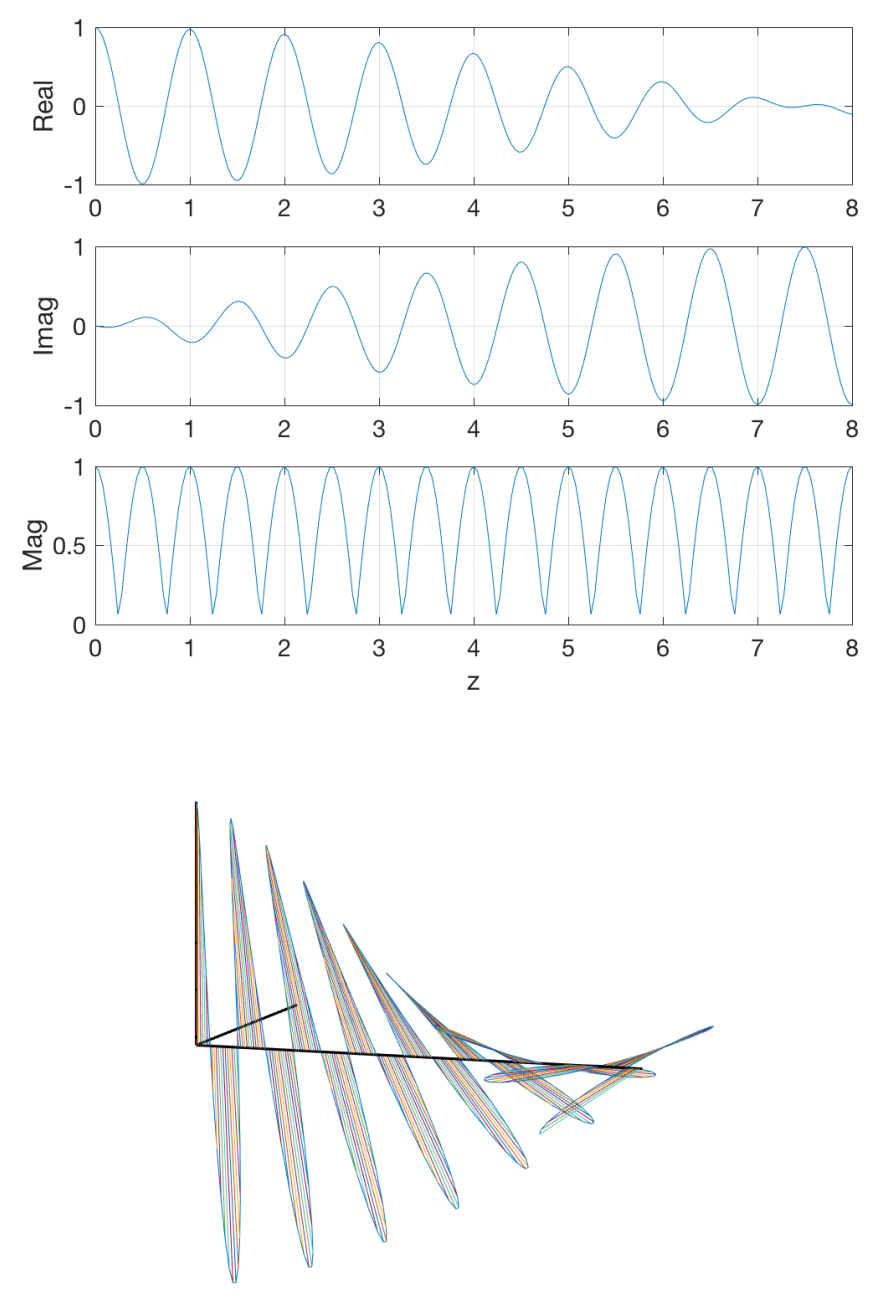

Figure B.3: Sum of complex exponentials: rotating cosine. Bottom 3-D plot shows complex value over $z$ : Left/right axis is $z$, other two are real/imaginary. 


\section{Appendix C}

\section{Multi-mode Rate Equation Model Phase}

\section{C.1 Multi-mode Phase}

This section will help get a better understanding of the multi-mode REM phase. The E-field phase is always the aggregate phase of the modes. The mode phase is always linear and the modes always come in pairs except for the fundamental. Because the modes are independent the relative phase between them will be random. This creates a seemingly chaotic phase for the total $E$-field. The phase is not random though and is typically piece-wise linear. A typical example is shown in figure 7.6. It is this aspect that we would like to explore.

\section{C.1.1 Symmetrical Modes}

If each of the mode pairs was perfectly symmetrical then they would cancel leaving only the fundamental mode. Because the mode pairs are complex conjugates of each other the sum is real. The mode pairs are not exactly the same frequency because they need to be integral multiples of the wavelengths that fit in the cavity, but they will be close. The phase of the 3 -mode REM is shown in figure C.1. The top shows the two symmetrical modes 2 and 3. Note that the plot shows the complex phase over time (plotted real versus imaginary over time). The bottom left is the fundamental and is constant at $1+0 i$. The bottom right shows a real sine wave (still in complex plane) shifted up by the fundamental. The complex parts of modes 2 and 3 have cancelled leaving the sine wave. If we were to calculate the phase of this sine wave it would switch from $+\pi$ to $-\pi$ every period. This square wave phase will come up again presently. The fundamental $(0 \mathrm{~Hz})$ does not affect the phase. This is because we have defined in this example that the carrier frequency be the fundamental mode frequency. The symmetry of the modes reveals then that we should expect the phase to be constant. 

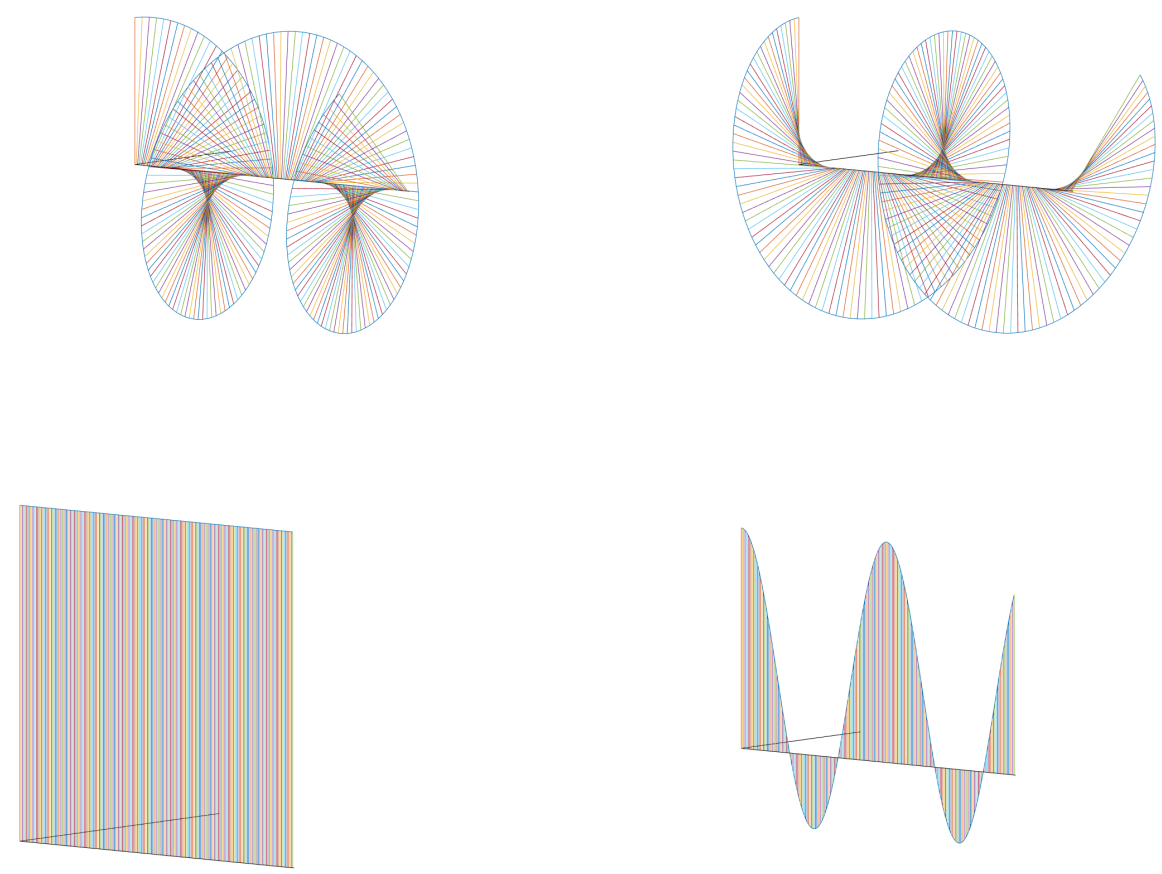

Figure C.1: 'Vector' plots of the complex envelope over time of individual modes and their sum for the 3-mode REM. Top: modes 2 and 3. Bottom: fundamental and sum. These are 3-D plots showing complex value over $z$ : Left/right axis is $z$, other two are real/imaginary.

\section{C.1.2 Mode Initial Phase}

Because of the mode-locking problem of the multi-mode REM we start each mode with a random phase as described in section 3.6. We will use a simplified model to explore the impact of the random phase. Instead of using the cavity to determine allowed frequencies and an MNA REM simulation we set the modes directly using complex exponentials. The mode frequencies are multiples of each other. We first note that no matter what initial phases or amplitudes we get the $+\pi,-\pi$ square wave phase. As the initial phases are changed the corners are rounded. An example is shown in figure C.2. It is important to note that this property of the $E$-field signal is small compared to any accumlated phase over time and will only be noticable when the phase is approximately constant. In cases where the phase does not accumulate we filter this out to see the accumulated phase only.

\section{C.1.3 Mode Amplitudes}

The amplitude of the mode pairs causes phase to accumulate at the rate of one of the modes. The amplitude combined with the initial phase offset form a complex number - a phasor. 

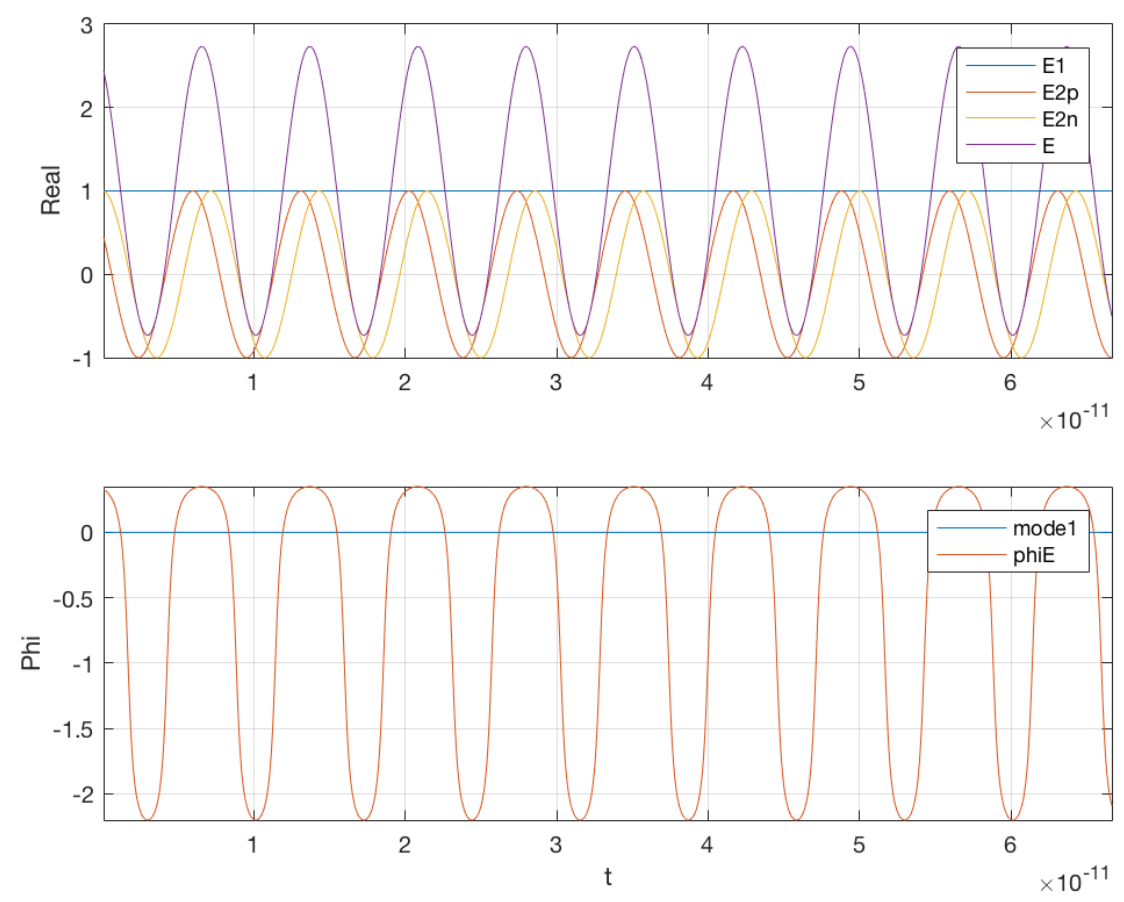

Figure C.2: Top: $E$-field as a sum of simple sinusoids with random initial phases. Bottom: Phase of $E$-field.

The equation for the $E$-field of the 5-mode REM is given in (C.1).

$$
\begin{aligned}
E & =A_{1} e^{i \phi_{1}}+A_{2} e^{i \triangle \omega_{2} t+i \phi_{2}}+A_{3} e^{i \triangle \omega_{2} t+i \phi_{2}}+A_{2} e^{-i \triangle \omega_{2} t+i \phi_{2}}+A_{3} e^{-i \triangle \omega_{3} t+i \phi_{3}} \\
& =A_{1} e^{i \phi_{1}}+A_{2} e^{i \phi_{2}} e^{i \triangle \omega_{2} t}+A_{3} e^{i \phi_{3}} e^{i \triangle \omega_{3} t}+A_{2} e^{i \phi_{2}} e^{-i \triangle \omega_{2} t}+A_{3} e^{i \phi_{3}} e^{-i \triangle \omega_{3} t}
\end{aligned}
$$

The $\triangle \omega$ 's are the difference between the actual mode frequency and our carrier; this is why mode 1 will always be $0[\mathrm{~Hz}]$ when we set the carrier to the fundamental mode of the cavity. When we move the initial phase out of the exponent we get the complex number that represents the phasor. We can draw a phasor diagram connecting them tip to tail and show the generation of the final $E$ vector. This is done in figure C.3. Not every configuration will accumulate phase; many simply cycle through a small range of phase. If the total $E$-field phasor keeps rotating in the same direction the phase will accumulate. To see the accumulated phase we must 'unwrap' the phase. Whenever the phasor sum crosses the $p i$ phase line there is a jump of $2 p i[\mathrm{rad}]$ and we know the phase is accumulating. The phasor sum example is shown over time in figure C.4. It shows the phase and the unwrapped phase. If this diagram were animated through time we would see the phasor rotating in the counterclockwise direction. Note that the total phase accumulates at the rate of mode 4 (negative of mode 2). When compared with the MNA REM simulation it compares extremely well.

In our simple sinusoid simulation each run has a different initial phase. All runs have an initial and final amplitude configuration where each mode has approximately the same 


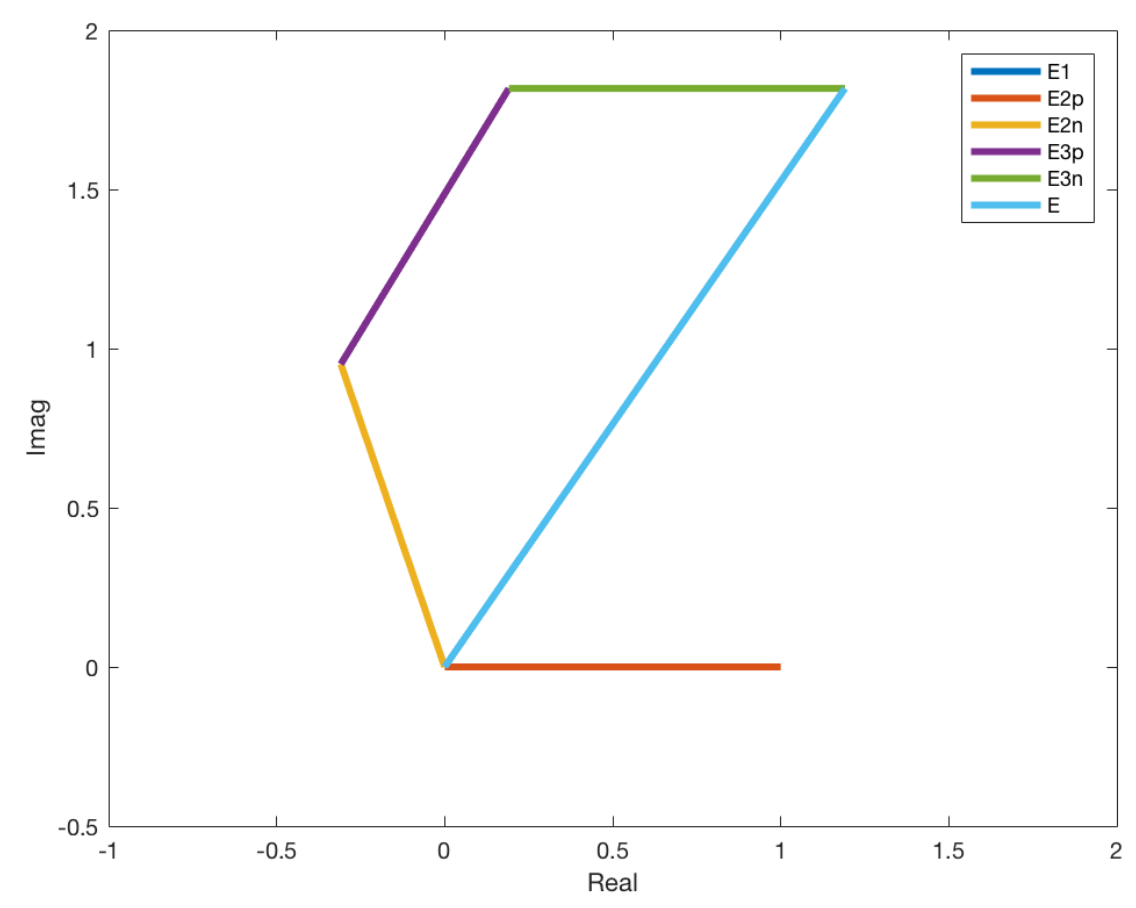

Figure C.3: E-field phase as a sum of phasors for a 5-mode REM. The phasors come in pairs which rotate in opposite directions.

amplitude. Between the initial and final amplitude configuration a typical one is used where the amplitude drops off on the pairs as the mode number increases. This will mimic the current pulse of the actual REM. We see the same pattern of line segments as we saw in figure 7.6. We can also see that the slope of the line segments matches one of the mode phase slopes. These match well with our multi-mode REM simulation shown in figure C.5. We see the total phase following mode 4 (negative frequency of mode 2). We can apply our knowledge of the $E$-fields being complex conjugate phasors (see figure C.1 and set the initial random phases to be symmetrical pairs. For example, if mode 2 has a phase of $\pi / 2$ we would set mode 4 (in a 5 -mode REM) to $-\pi / 2$. This will cancel the initial phases and we will get a zero phase. This is shown in the right side of figure C.7.

These results support our idea that the amplitude configuration determines the phase. Once the laser reaches threshold and is lasing the amplitudes are in a different but fixed configuration. The total phase is determined by the initial phases and the amplitude configuration. 

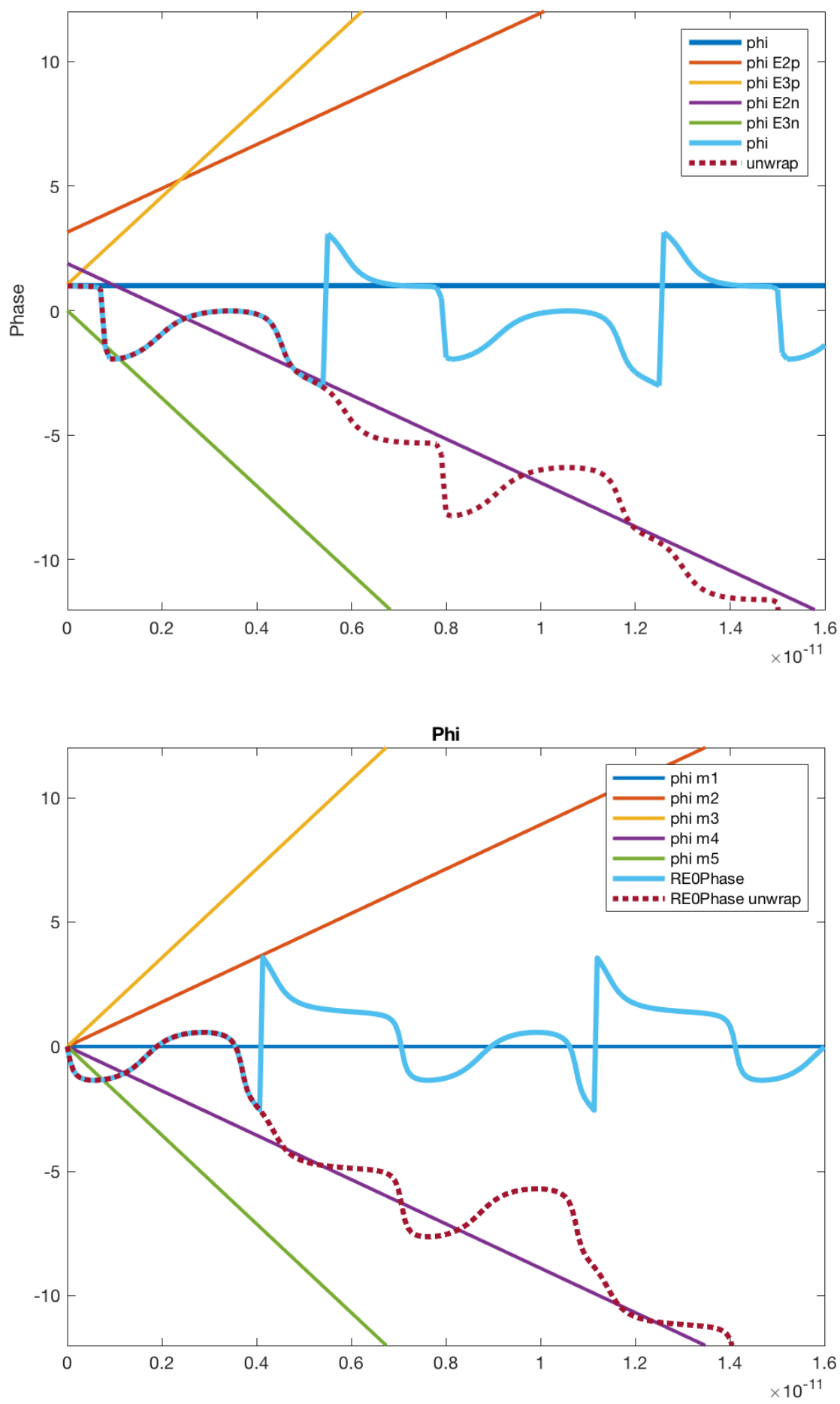

Figure C.4: E-field phase of a 5-mode REM showing phase accumulating with mode 4.Left: simple simulation. Right: MNA multi-mode REM simulation. 

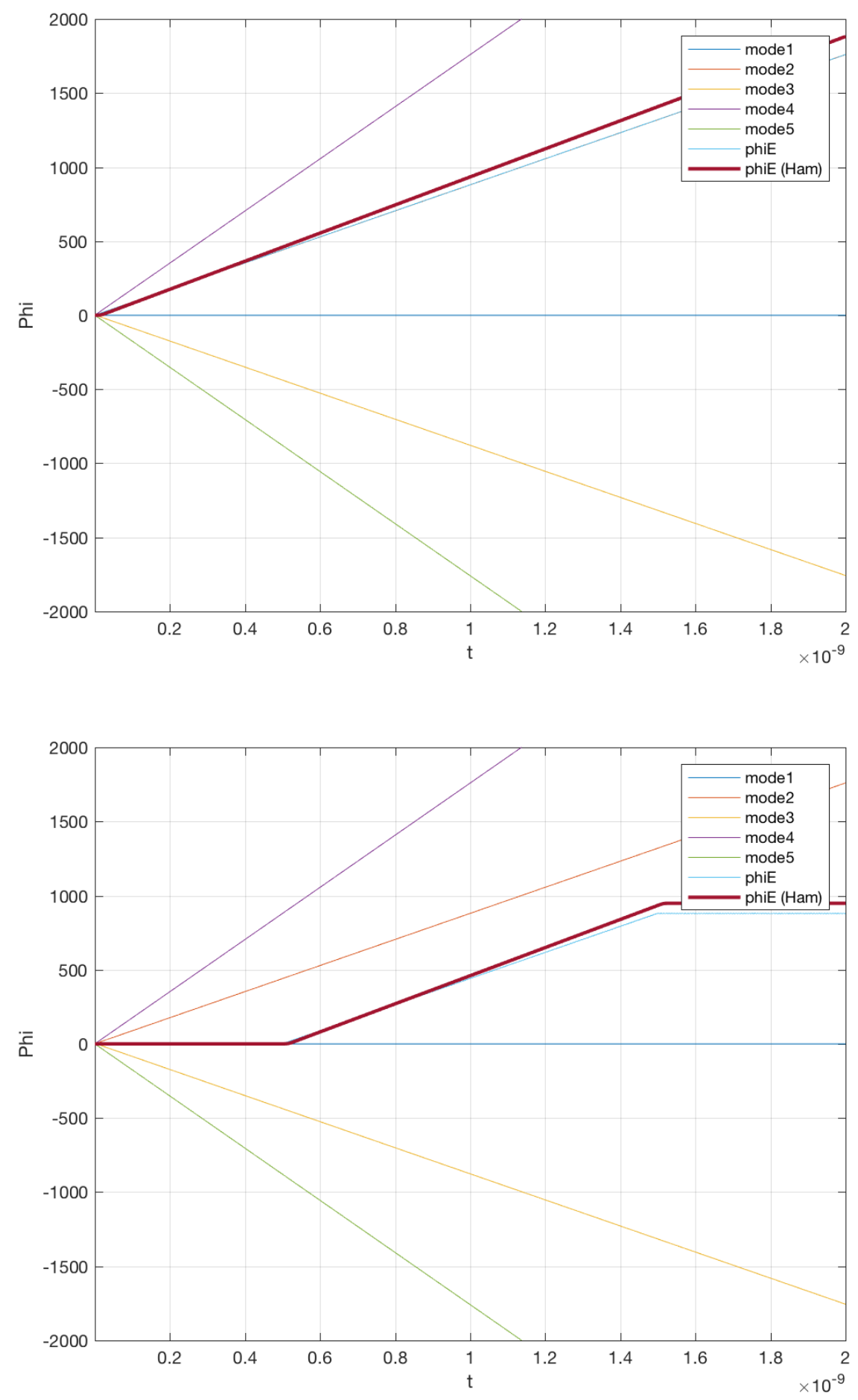

Figure C.5: E-field phase as a sum of simple sinusoids for a 5-mode REM. Each run has the same initial and final amplitude configuration but with different initial phases. In the middle a typical mode distribution is used. 

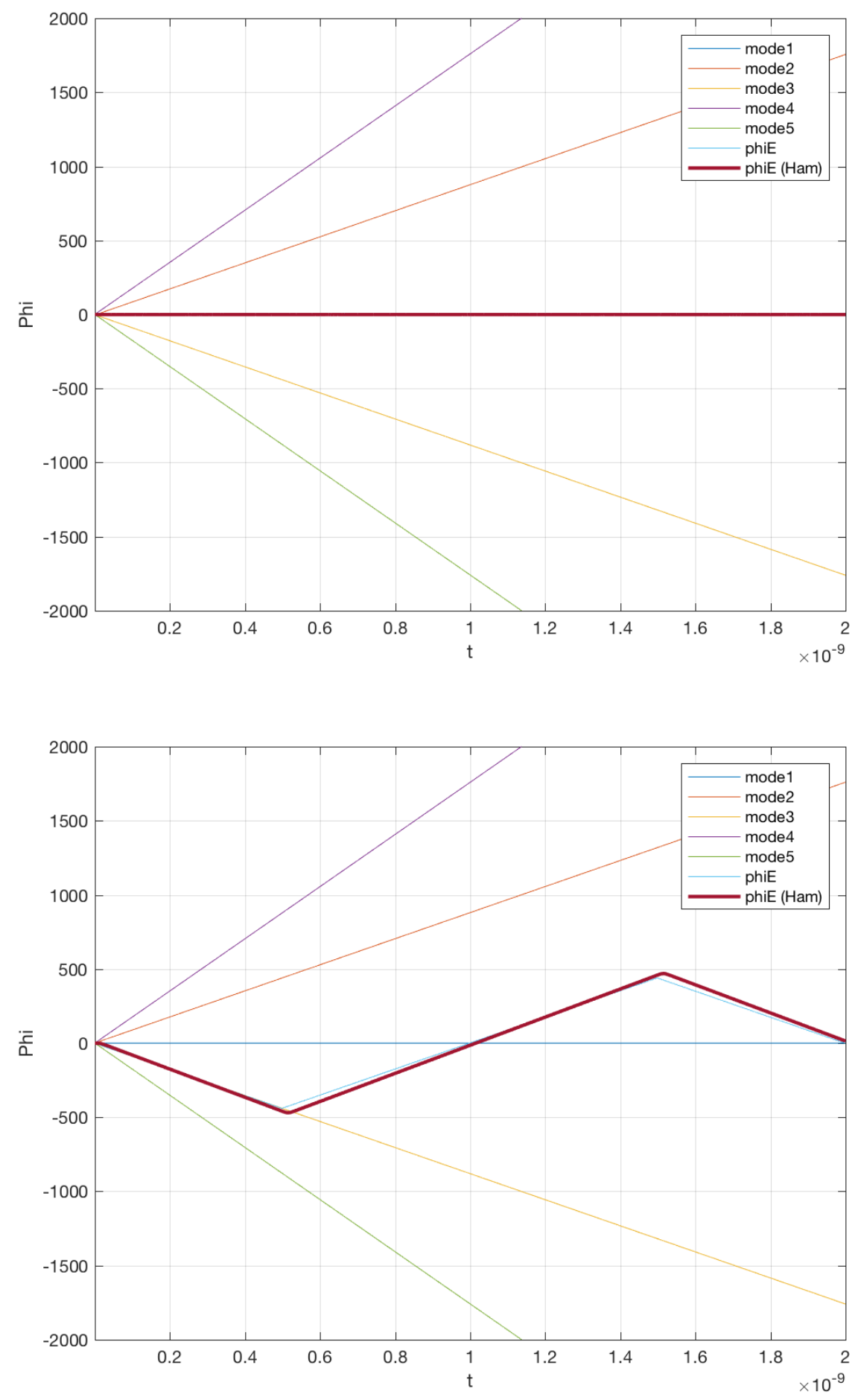

Figure C.6: E-field phase as a sum of simple sinusoids for a 5-mode REM. Each run has the same initial and final amplitude configuration but with different initial phases. In the middle a typical mode distribution is used. 

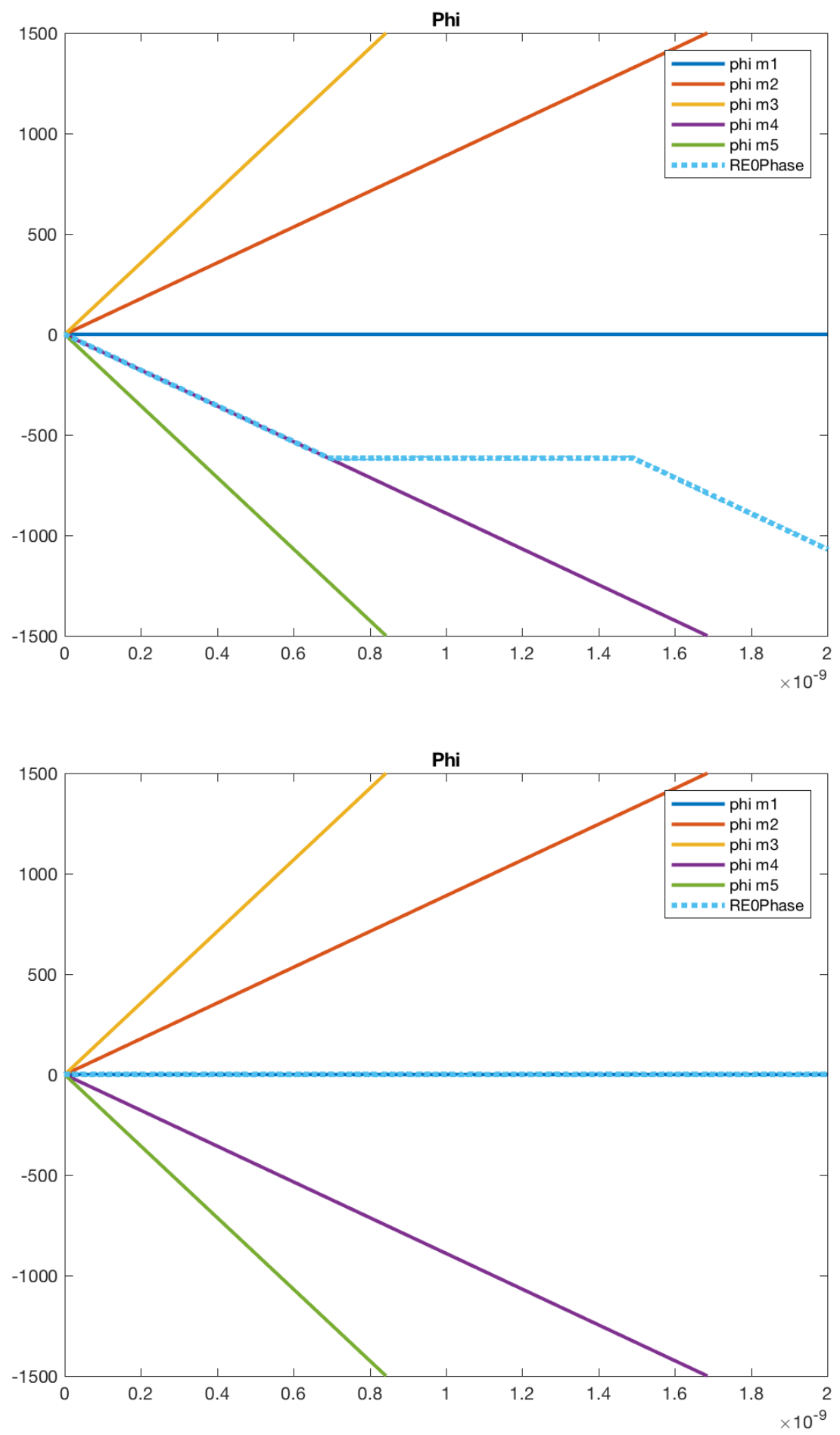

Figure C.7: E-field phase for a 5-mode REM. Left: random initial phases. Right: random phases but symmetrical. 


\section{Appendix D}

\section{Henry Rate Equations}

\section{D.1 Rate Equations for Photons and Phase}

In his paper [10] Henry does not include the the carrier equation. His rate equations for photons and phase are given by (D.1) and (D.2) respectively.

$$
\begin{aligned}
\dot{\phi} & =\frac{\alpha}{2}(G-\gamma) \\
\dot{I} & =(G-\gamma) I
\end{aligned}
$$

where $G$ is the net rate of stimulated emission and $\gamma$ is the rate of cavity loss (facet waveguide).

To put them in a more familiar form let $I=S$ equal the average number of photons in the cavity and $\gamma=\frac{1}{\tau_{p}}$ which is the rate of loss in the cavity. They become

$$
\begin{aligned}
\frac{d \phi}{d t} & =\frac{\alpha}{2}\left(G-\frac{1}{\tau_{p}}\right) \\
\frac{d S}{d t} & =\left(G-\frac{1}{\tau_{p}}\right) S
\end{aligned}
$$

Comparing these with (3.11) and (3.12) we see that they are $\phi$ and photon equations. The photon equation does not include the spontaneous emission term and the specific dependence on $N$ is hidden. The confinement factor is also neglected. Note that this is the phase of the slowly varying envelope, and not the phase of the full signal including the carrier. Also because this is a lumped model the variable $\phi$ represents the phase at a single point in the active medium which is taken to be the output. 


\section{Appendix E}

\section{Derivation of Henry Photon and Phase Equations}

\section{E.1 Henry's Derivation of the Rate Equations for $I$ and $\phi$}

We follow the derivation given in the appendix of [10]. This paper is often referenced when dealing with the REM and is why often the alpha factor $\left(\alpha_{H}\right)$ is called the Henry factor and why we subscript it with $H$. The $I$ in these equations is the intensity term and is the same as our photon density term $S$. For a relationship between $S$ and $E$, the electric field we have

$$
S=E \cdot E^{*}
$$

where the ${ }^{* *}$ ' denotes the complex conjugate.

Starting with the 1-D 2nd-order wave equation

$$
\frac{\partial^{2} E}{\partial z^{2}}=\frac{1}{v^{2}} \frac{\partial^{2}}{\partial t^{2}} E
$$

and letting $v=\frac{c}{n}$ we get

$$
\frac{\partial^{2} E}{\partial z^{2}}=\frac{n^{2}}{c^{2}} \frac{\partial^{2}}{\partial t^{2}} E
$$

We can replace $n^{2}=\varepsilon$ (the dielectric constant or relative permittivity) we get the wave equation for the electric field

$$
\frac{\partial^{2} E}{\partial z^{2}}=\frac{1}{c^{2}} \frac{\partial^{2}}{\partial t^{2}}(\varepsilon E)
$$

where

$$
E \sim \beta e^{i(\omega t-k z)}+\beta^{*} e^{-i(\omega t-k z)}
$$

Adding the complex conjugate makes $E$ real. Therefore

$$
\varepsilon E \sim \varepsilon \beta e^{i(\omega t-k z)}+\varepsilon \beta^{*} e^{-i(\omega t-k z)}
$$


Then "to take into account that the change in the dielectric constant $\varepsilon$ due to disperson, caused by the time dependence of $\beta$ ", Henry writes

$$
\varepsilon E \sim\left(\varepsilon(\omega) \beta+i \frac{\partial \varepsilon}{\partial \omega} \dot{\beta}\right) e^{i(\omega t-k z)}+\text { c.c. }
$$

Making the substitution

$$
\varepsilon \beta=\varepsilon(\omega) \beta+i \frac{\partial \varepsilon}{\partial \omega} \dot{\beta}
$$

This substitution is looked at in section E.2.1. Note that if one assumes $\varepsilon$ is constant the same result is obtained for the RHS. Refer to section E.2.3.

Then he substitutes (E.2) and (E.3) into (E.1). This calculation is done in section E.2.2 giving

$$
\frac{2 i \omega}{c^{2}}\left(\varepsilon-\frac{\omega}{2} \frac{\partial \varepsilon}{\partial \omega}\right) \dot{\beta}=\left(\varepsilon \frac{\omega^{2}}{c^{2}}-k^{2}\right) \beta
$$

which does not match (E.4)! If we use, however, the linear approximation (E.21) developed in E.2.1 instead we get

$$
\frac{2 i \omega}{c^{2}}\left(\varepsilon+\frac{\omega}{2} \frac{\partial \varepsilon}{\partial \omega}\right) \dot{\beta}=\left(\frac{\omega^{2}}{c^{2}} \varepsilon-k^{2}\right) \beta
$$

as expected.

\section{E.1.1 Net Gain}

The medium gain is determined by the attenuation coefficient (calculated in section E.2.11).

$$
\alpha_{a t t}=2 k^{\prime \prime}=-\frac{2 \omega}{c} n^{\prime \prime}
$$

Note that for positive extinction factor $n^{\prime \prime}$, the attenuation coefficient $\alpha$ will be negative for attenuation. This will be the net gain

$$
g-\alpha_{\gamma} \equiv-\frac{2 \omega}{c} n^{\prime \prime}
$$

This is related to the term $G-\gamma$ in (D.1),(D.2) by (E.11). Note at threshold $g=\alpha_{\gamma}$. Henry then says that at threshold $\epsilon$ is real. This means that Henry is considering $\epsilon$ of the system, not the material.

Henry then says $\omega$ is chosen so

$$
\frac{\omega}{c} n^{\prime}=k
$$

presuming that he means the real part of $k$. Interesting as this sounds like it is not necessarily the actual value of $\omega$. 
Complex permitivity is calculated in E.2.4 and is given by

$$
\begin{aligned}
\varepsilon_{r} & =\left(n^{\prime}\right)^{2}+2 n^{\prime}\left(\triangle n^{\prime}-i \triangle n^{\prime \prime}\right) \\
& =\left(n^{\prime}\right)^{2}+2 n^{\prime} \triangle n^{\prime \prime}\left(\frac{\triangle n^{\prime}}{\triangle n^{\prime \prime}}-i\right) \\
& =\left(n^{\prime}\right)^{2}+2 n^{\prime} \triangle n^{\prime \prime}(\alpha-i) \\
& =\left(n^{\prime}\right)^{2}-2 i n^{\prime} \triangle n^{\prime \prime}(1+i \alpha)
\end{aligned}
$$

where we define

$$
\alpha \equiv \frac{\triangle n^{\prime}}{\triangle n^{\prime \prime}}
$$

Then

$$
\varepsilon+\frac{\omega}{2} \frac{\partial \varepsilon}{\partial \omega}=n^{\prime}\left(n^{\prime}+\omega \frac{d n^{\prime}}{d \omega}\right)=\frac{c}{v_{g}} n^{\prime}
$$

The calculation for (E.9) is done in E.2.6.

Substituting (E.6), (E.7), and (E.9) into (E.4) we find

$$
\dot{\beta}=\frac{\left(g-\alpha_{\gamma}\right) v_{g}}{2}(1+i \alpha) \beta
$$

The calculation for (E.10) is done in E.2.7.

"In a dispersive medium, the ratio of energy flux to energy density is the group velocity $v_{g}[39] "$. So we get

$$
G-\gamma=v_{g}\left(g-\alpha_{\gamma}\right)
$$

Therefore

$$
\dot{\beta}=\left(\frac{G-\gamma}{2}\right)(1+i \alpha) \beta
$$

and

$$
\dot{\beta}^{*}=\left(\frac{G-\gamma}{2}\right)(1-i \alpha) \beta^{*}
$$

Transform to the $I, \phi$ representation using

$$
\begin{gathered}
I=\beta \beta^{*} \\
\phi=\frac{1}{2 i} \ln \left(\frac{\beta}{\beta^{*}}\right)
\end{gathered}
$$

(E.15) derived in section E.2.8.

We then find

$$
\dot{I}=(G-\gamma) I
$$

which is derived in section E.2.9 and

$$
\dot{\phi}=\frac{\alpha}{2}(G-\gamma)
$$

which is derived in section E.2.10. 


\section{E.2 Derivations}

\section{E.2.1 Linear Approximation to $\epsilon(\omega) \beta(t)$}

$$
\epsilon E \sim \epsilon \beta e^{i(\omega t-k z)}+\text { c.c. }
$$

In (E.3) Henry uses this substitution

$$
\epsilon(\omega) \beta(t)=\epsilon(\omega) \beta+i \frac{\partial \epsilon}{\partial \omega} \dot{\beta}
$$

If we choose an operating point $\omega=\omega_{1}$, then with respect to that operating point, we can linearly approximate

$$
\epsilon(\omega)=\epsilon_{1}+\frac{\partial \epsilon}{\partial \omega} \triangle \omega
$$

where $\epsilon_{1}=\epsilon\left(\omega_{1}\right)$ and $\Delta \omega=\omega-\omega_{1}$. If we assume $\beta(t)=\beta_{1} e^{i \Delta \omega t}$ then

$$
\frac{\partial \beta}{\partial t}=i \beta_{1} \triangle \omega e^{i \triangle \omega t}=i \triangle \omega \beta(t)
$$

and from this we get

$$
\triangle \omega \simeq \frac{1}{i \beta(t)} \frac{\partial \beta}{\partial t}=-\frac{i}{\beta(t)} \frac{\partial \beta}{\partial t}
$$

Multiplying (E.19) by $\beta(t)$ gives

$$
\begin{aligned}
\epsilon(\omega) \beta(t) & =\left(\epsilon_{1}+\frac{\partial \epsilon}{\partial \omega} \triangle \omega\right) \beta(t) \\
& =\epsilon_{1} \beta(t)+\frac{\partial \epsilon}{\partial \omega} \triangle \omega \beta(t)
\end{aligned}
$$

Substitute in for $\triangle \omega$ using (E.20)

$$
\begin{aligned}
\epsilon(\omega) \beta(t) & =\epsilon_{1} \beta(t)+\frac{\partial \epsilon}{\partial \omega}\left(-\frac{i}{\beta(t)} \frac{\partial \beta}{\partial t}\right) \beta(t) \\
& =\epsilon_{1} \beta(t)-i \frac{\partial \epsilon}{\partial \omega} \dot{\beta}
\end{aligned}
$$

This does not agree with the substitution Henry made: see (E.18). 


\section{E.2.2 Calculation of Henry (A4)}

Check Henry calculation of (E.4). Starting with LHS of (E.1)

$$
\begin{aligned}
\text { L.H.S. } & =\frac{\partial^{2} E}{\partial z^{2}} \\
& =\frac{\partial^{2}}{\partial z^{2}}\left(\beta e^{i(\omega t-k z)}+\beta^{*} e^{-i(\omega t-k z)}\right) \\
& =\frac{\partial}{\partial z}\left(\beta(-i k) e^{i(\omega t-k z)}+\beta^{*}(i k) e^{-i(\omega t-k z)}\right) \\
& =-k^{2}\left(\beta e^{i(\omega t-k z)}+\beta^{*} e^{-i(\omega t-k z)}\right) \\
& =-k^{2} E
\end{aligned}
$$

then the RHS of (E.1) using (E.18)

$$
\begin{aligned}
\text { R.H.S. } & =\frac{1}{c^{2}} \frac{\partial^{2}}{\partial t^{2}}(\varepsilon E) \\
& =\frac{1}{c^{2}} \frac{\partial^{2}}{\partial t^{2}}\left(\left[\varepsilon \beta+i \frac{\partial \varepsilon}{\partial \omega} \dot{\beta}\right] e^{i(\omega t-k z)}+c . c .\right) \\
& =\frac{1}{c^{2}} \frac{\partial}{\partial t}\left\{\frac{\partial}{\partial t}\left[\varepsilon \beta+i \frac{\partial \varepsilon}{\partial \omega} \dot{\beta}\right] e^{i(\omega t-k z)}+\left[\varepsilon \beta+i \frac{\partial \varepsilon}{\partial \omega} \dot{\beta}\right] \frac{\partial}{\partial t}\left(e^{i(\omega t-k z)}\right)+c . c .\right\} \\
& =\frac{1}{c^{2}} \frac{\partial}{\partial t}\left\{\left[\varepsilon \dot{\beta}+i \frac{\partial \varepsilon}{\partial \omega} \ddot{\beta}\right] e^{i(\omega t-k z)}+\left[\varepsilon \beta+i \frac{\partial \varepsilon}{\partial \omega} \dot{\beta}\right](i \omega)\left(e^{i(\omega t-k z)}\right)+c . c .\right\} \\
& =\frac{1}{c^{2}} \frac{\partial}{\partial t}\left\{\left[\varepsilon \dot{\beta}+i \frac{\partial \varepsilon}{\partial \omega} \ddot{\beta}+i \omega \varepsilon \beta-\omega \frac{\partial \varepsilon}{\partial \omega} \dot{\beta}\right] e^{i(\omega t-k z)}+c . c .\right\}
\end{aligned}
$$

Dropping derivatives of $\beta$ higher than first-order

$$
\begin{aligned}
\text { R.H.S. } & =\frac{1}{c^{2}} \frac{\partial}{\partial t}\left\{\left[\varepsilon \dot{\beta}+i \omega \varepsilon \beta-\omega \frac{\partial \varepsilon}{\partial \omega} \dot{\beta}\right] e^{i(\omega t-k z)}+c . c .\right\} \\
& =\frac{1}{c^{2}} \frac{\partial}{\partial t}\left[\varepsilon \dot{\beta}+i \omega \varepsilon \beta-\omega \frac{\partial \varepsilon}{\partial \omega} \dot{\beta}\right] e^{i(\omega t-k z)}+ \\
& \frac{1}{c^{2}}\left[\varepsilon \dot{\beta}+i \omega \varepsilon \beta-\omega \frac{\partial \varepsilon}{\partial \omega} \dot{\beta}\right] \frac{\partial}{\partial t}\left(e^{i(\omega t-k z)}\right)+\text { c.c. }
\end{aligned}
$$


Again dropping derivatives of $\beta$ higher than first order (don't even write them)

$$
\begin{aligned}
\text { R.H.S. } & =\frac{1}{c^{2}}\left\{i \omega \varepsilon \dot{\beta} e^{i(\omega t-k z)}+\left[\varepsilon \dot{\beta}+i \omega \varepsilon \beta-\omega \frac{\partial \varepsilon}{\partial \omega} \dot{\beta}\right](i \omega) e^{i(\omega t-k z)}+c . c .\right\} \\
& =\frac{1}{c^{2}}\left\{\left[i \omega \varepsilon \dot{\beta}+i \omega \varepsilon \dot{\beta}-\omega^{2} \varepsilon \beta-i \omega^{2} \frac{\partial \varepsilon}{\partial \omega} \dot{\beta}\right] e^{i(\omega t-k z)}+c . c .\right\} \\
& =\frac{1}{c^{2}}\left\{\left[2 i \omega \varepsilon \dot{\beta}-\omega^{2} \varepsilon \beta-i \omega^{2} \frac{\partial \varepsilon}{\partial \omega} \dot{\beta}\right] e^{i(\omega t-k z)}+c . c .\right\} \\
& =\frac{1}{c^{2}}\left\{\left[2 i \omega\left(\varepsilon-\frac{\omega}{2} \frac{\partial \varepsilon}{\partial \omega}\right) \dot{\beta}-\omega^{2} \varepsilon \beta\right] e^{i(\omega t-k z)}+c . c .\right\} \\
& =\frac{1}{c^{2}}\left\{\left[2 i \omega \varepsilon \dot{\beta}-i \omega^{2} \frac{\partial \varepsilon}{\partial \omega} \dot{\beta}-\omega^{2} \varepsilon \beta\right]\right\} e^{i(\omega t-k z)}+c . c . \\
& =\frac{1}{c^{2}}\left\{\varepsilon\left[2 i \omega \dot{\beta}-\omega^{2} \beta\right]-i \omega^{2} \frac{\partial \varepsilon}{\partial \omega} \dot{\beta}\right\} e^{i(\omega t-k z)}+c . c .
\end{aligned}
$$

Setting the $\beta$ terms on both sides equal

$$
\begin{aligned}
-k^{2} \beta e^{i(\omega t-k z)} & =\frac{1}{c^{2}}\left\{\varepsilon\left[2 i \omega \dot{\beta}-\omega^{2} \beta\right]-i \omega^{2} \frac{\partial \varepsilon}{\partial \omega} \dot{\beta}\right\} e^{i(\omega t-k z)} \\
-k^{2} \beta & =\frac{1}{c^{2}}\left\{2 i \varepsilon \omega \dot{\beta}-\varepsilon \omega^{2} \beta-i \omega^{2} \frac{\partial \varepsilon}{\partial \omega} \dot{\beta}\right\} \\
-k^{2} \beta & =\frac{1}{c^{2}} 2 i \varepsilon \omega \dot{\beta}-\frac{1}{c^{2}} \varepsilon \omega^{2} \beta-\frac{1}{c^{2}} i \omega^{2} \frac{\partial \varepsilon}{\partial \omega} \dot{\beta} \\
\left(\frac{\varepsilon \omega^{2}}{c^{2}}-k^{2}\right) \beta & =\frac{2 i \omega}{c^{2}}\left(\varepsilon-\frac{\omega}{2} \frac{\partial \varepsilon}{\partial \omega}\right) \dot{\beta}
\end{aligned}
$$

where we have collected $\beta$ and $\dot{\beta}$ terms. Note that this does not agree with (E.4)!

\section{Repeat Using Linear Approximation Substitution}

Repeat keeping the LHS the same but using the linear approximation substitution (E.21) derived in E.2.1 for the RHS.

$$
\begin{aligned}
\text { L.H.S. } & =-k^{2} E \\
& =-k^{2} \beta e^{i \omega t-k z}+c . c .
\end{aligned}
$$




$$
\begin{aligned}
\text { R.H.S. } & =\frac{1}{c^{2}} \frac{\partial^{2}}{\partial t^{2}}(\varepsilon E) \\
& =\frac{1}{c^{2}} \frac{\partial^{2}}{\partial t^{2}}\left(\left[\varepsilon \beta-i \frac{\partial \varepsilon}{\partial \omega} \dot{\beta}\right] e^{i(\omega t-k z)}+c . c .\right) \\
& =\frac{1}{c^{2}} \frac{\partial}{\partial t}\left\{\frac{\partial}{\partial t}\left[\varepsilon \beta-i \frac{\partial \varepsilon}{\partial \omega} \dot{\beta}\right] e^{i(\omega t-k z)}+\left[\varepsilon \beta-i \frac{\partial \varepsilon}{\partial \omega} \dot{\beta}\right] \frac{\partial}{\partial t}\left(e^{i(\omega t-k z)}\right)+c . c .\right\} \\
& =\frac{1}{c^{2}} \frac{\partial}{\partial t}\left\{\left[\varepsilon \dot{\beta}-i \frac{\partial \varepsilon}{\partial \omega} \ddot{\beta}\right] e^{i(\omega t-k z)}+\left[\varepsilon \beta-i \frac{\partial \varepsilon}{\partial \omega} \dot{\beta}\right](i \omega)\left(e^{i(\omega t-k z)}\right)+c . c .\right\} \\
& =\frac{1}{c^{2}} \frac{\partial}{\partial t}\left\{\left[\varepsilon \dot{\beta}-i \frac{\partial \varepsilon}{\partial \omega} \ddot{\beta}+i \omega \varepsilon \beta+\omega \frac{\partial \varepsilon}{\partial \omega} \dot{\beta}\right] e^{i(\omega t-k z)}+c . c .\right\}
\end{aligned}
$$

Dropping derivatives of $\beta$ higher than first-order

$$
\begin{aligned}
\text { R.H.S. } & =\frac{1}{c^{2}} \frac{\partial}{\partial t}\left\{\left[\varepsilon \dot{\beta}+i \omega \varepsilon \beta+\omega \frac{\partial \varepsilon}{\partial \omega} \dot{\beta}\right] e^{i(\omega t-k z)}+c . c .\right\} \\
& =\frac{1}{c^{2}} \frac{\partial}{\partial t}\left[\varepsilon \dot{\beta}+i \omega \varepsilon \beta+\omega \frac{\partial \varepsilon}{\partial \omega} \dot{\beta}\right] e^{i(\omega t-k z)}+ \\
& \frac{1}{c^{2}}\left[\varepsilon \dot{\beta}+i \omega \varepsilon \beta+\omega \frac{\partial \varepsilon}{\partial \omega} \dot{\beta}\right] \frac{\partial}{\partial t}\left(e^{i(\omega t-k z)}\right)+c . c .
\end{aligned}
$$

Again dropping derivatives of $\beta$ higher than first order (don't even write them)

$$
\begin{aligned}
\text { R.H.S. } & =\frac{1}{c^{2}}\left\{i \omega \varepsilon \dot{\beta} e^{i(\omega t-k z)}+\left[\varepsilon \dot{\beta}+i \omega \varepsilon \beta+\omega \frac{\partial \varepsilon}{\partial \omega} \dot{\beta}\right](i \omega) e^{i(\omega t-k z)}+c . c .\right\} \\
& =\frac{1}{c^{2}}\left\{\left[i \omega \varepsilon \dot{\beta}+i \omega \varepsilon \dot{\beta}-\omega^{2} \varepsilon \beta+i \omega^{2} \frac{\partial \varepsilon}{\partial \omega} \dot{\beta}\right] e^{i(\omega t-k z)}+c . c .\right\} \\
& =\frac{1}{c^{2}}\left\{\left[2 i \omega \varepsilon \dot{\beta}-\omega^{2} \varepsilon \beta+i \omega^{2} \frac{\partial \varepsilon}{\partial \omega} \dot{\beta}\right] e^{i(\omega t-k z)}+c . c .\right\} \\
& =\frac{1}{c^{2}}\left\{\varepsilon\left[2 i \omega \dot{\beta}-\omega^{2} \beta\right]+i \omega^{2} \frac{\partial \varepsilon}{\partial \omega} \dot{\beta}\right\} e^{i(\omega t-k z)}+c . c .
\end{aligned}
$$

Setting the LHS equal to RHS

$$
\begin{aligned}
-k^{2} \beta e^{i(\omega t-k z)} & =\frac{1}{c^{2}}\left\{\varepsilon\left[2 i \omega \dot{\beta}-\omega^{2} \beta\right]+i \omega^{2} \frac{\partial \varepsilon}{\partial \omega} \dot{\beta}\right\} e^{i(\omega t-k z)} \\
-k^{2} \beta & =\frac{1}{c^{2}}\left\{2 i \varepsilon \omega \dot{\beta}-\varepsilon \omega^{2} \beta+i \omega^{2} \frac{\partial \varepsilon}{\partial \omega} \dot{\beta}\right\} \\
-k^{2} \beta & =\frac{1}{c^{2}} 2 i \varepsilon \omega \dot{\beta}-\frac{1}{c^{2}} \varepsilon \omega^{2} \beta+\frac{1}{c^{2}} i \omega^{2} \frac{\partial \varepsilon}{\partial \omega} \dot{\beta} \\
\left(\frac{\varepsilon \omega^{2}}{c^{2}}-k^{2}\right) \beta & =\frac{2 i \omega}{c^{2}}\left(\varepsilon+\frac{\omega}{2} \frac{\partial \varepsilon}{\partial \omega}\right) \dot{\beta}
\end{aligned}
$$

where we have collected $\beta$ and $\dot{\beta}$ terms. Note that this does agree with (E.4). 


\section{E.2.3 Calculation of Henry (A4) with constant $\varepsilon$}

Check Henry calculation of (E.4). The LHS is the same:

$$
\text { L.H.S. }=-k^{2} E
$$

\section{E.2.4 Calculation of Henry (A7): Relation to $\epsilon$ and $n$}

Develop the relationship between the complex permitivity and the complex index of refraction.

We know that in a non-magnetic material [9], p $213 \varepsilon=\varepsilon_{0}(1+\chi)$ and $n^{2}=1+\chi$ and complex refractive index $n=n^{\prime}-i n^{\prime \prime}$ so

$$
\varepsilon=\varepsilon_{0} n^{2}
$$

Note: discrepency between $\varepsilon$ and $\varepsilon_{r}$. Assume when Henry says $\varepsilon$ he means the relative permititivy $\varepsilon_{r}$.

\section{E.2.5 Assume start at threshold}

Let $n^{\prime \prime}=0$ and we get $n=n^{\prime}+\triangle n^{\prime}-i \triangle n^{\prime \prime}$ so

$$
\begin{aligned}
\epsilon^{\prime}-i \epsilon^{\prime \prime} & =\left(n^{\prime}+\triangle n^{\prime}\right)^{2}-\triangle n^{\prime \prime 2}-i 2\left(n^{\prime}+\triangle n^{\prime}\right) \triangle n^{\prime \prime} \\
& =\left(n^{\prime}\right)^{2}+2 n^{\prime} \triangle n^{\prime}+\triangle n^{2}-\triangle n^{\prime 2}-2 i n^{\prime} \triangle n^{\prime \prime}-2 i \triangle n^{\prime} \triangle n^{\prime \prime} \\
& =\left(n^{\prime}\right)^{2}+2 n^{\prime}\left(\triangle n^{\prime}-i \triangle n^{\prime \prime}\right)+\triangle n^{2}-\triangle n^{\prime \prime 2}-2 i \triangle n^{\prime} \triangle n^{\prime \prime} \\
& =\left(n^{\prime}\right)^{2}+2 n^{\prime}\left(\triangle n^{\prime}-i \triangle n^{\prime \prime}\right)
\end{aligned}
$$

where in the last step we neglect terms with $\triangle n^{2}$ (the last three).

\section{E.2.6 Calculation of Henry (A9)}

The bracketed term on left side of (E.4) is related to the group velocity $v_{g}$. Here for convenience we repeat (E.4).

$$
\frac{2 i \omega}{c^{2}}\left(\varepsilon+\frac{\omega}{2} \frac{\partial \varepsilon}{\partial \omega}\right) \dot{\beta}=\left(\frac{\omega^{2}}{c^{2}} \varepsilon-k^{2}\right) \beta
$$

From [9] (1.5-10) (assuming $\left.n \Rightarrow n^{\prime}\right)$

$$
v_{g}=\frac{c}{n^{\prime}+\omega \frac{d n^{\prime}}{d \omega}}
$$

so

$$
n^{\prime}+\omega \frac{d n^{\prime}}{d \omega}=\frac{c}{v_{g}}
$$


From (E.7)

$$
\epsilon=\left(n^{\prime}\right)^{2}-2 i n^{\prime} \triangle n^{\prime \prime}(1+i \alpha)
$$

so

$$
\frac{\partial \epsilon}{\partial \omega}=\frac{\partial}{\partial \omega}\left\{\left(n^{\prime}\right)^{2}-2 i n^{\prime} \triangle n^{\prime \prime}(1+i \alpha)\right\}=\left\{2 n^{\prime}-2 i \triangle n^{\prime \prime}(1+i \alpha)\right\} \frac{\partial n^{\prime}}{\partial \omega}
$$

Starting with bracketed term on left side of (E.4) if we sub in $\epsilon_{r}=\left(n^{\prime}\right)^{2},($ E.23), and (E.22) we get

$$
\begin{aligned}
\varepsilon+\frac{\omega}{2} \frac{\partial \varepsilon}{\partial \omega} & =\left(n^{\prime}\right)^{2}-2 i n^{\prime} \triangle n^{\prime \prime}(1+i \alpha)+\frac{\omega}{2}\left\{2 n^{\prime}-2 i \triangle n^{\prime \prime}(1+i \alpha)\right\} \frac{\partial n^{\prime}}{\partial \omega} \\
& =\left(n^{\prime}\right)^{2}-2 i n^{\prime} \triangle n^{\prime \prime}(1+i \alpha)+\omega\left\{n^{\prime}-i \triangle n^{\prime \prime}(1+i \alpha)\right\} \frac{\partial n^{\prime}}{\partial \omega}
\end{aligned}
$$

But if we ignore the second term in (E.7) we get

$$
\frac{\partial \epsilon}{\partial \omega}=2 n^{\prime} \frac{\partial n^{\prime}}{\partial \omega}
$$

And then the bracketed term on left side of (E.4) after sub in $\epsilon_{r}=n^{2}$, (E.23), and eqrefeq:n-plus-w-dn-dw gives us

$$
\varepsilon+\frac{\omega}{2} \frac{\partial \varepsilon}{\partial \omega}=n^{\prime 2}+\frac{\omega}{2} 2 n^{\prime} \frac{\partial n^{\prime}}{\partial \omega}=n^{\prime}\left(n^{\prime}+\omega \frac{\partial n^{\prime}}{\partial \omega}\right)=\frac{c}{v_{g}} n^{\prime}
$$

which agrees with (E.9) Henry (A9)

$$
\varepsilon+\frac{\omega}{2} \frac{\partial \varepsilon}{\partial \omega}=n^{\prime}\left(n^{\prime}+\omega \frac{d n^{\prime}}{d \omega}\right)=\frac{c}{v_{g}} n^{\prime}
$$

\section{E.2.7 Calculation for Henry (A10)}

Substitute (E.6)

$$
k=\frac{\omega}{c} n^{\prime}
$$

$$
\epsilon=n^{\prime 2}-2 i n^{\prime} \triangle n^{\prime \prime}(1+i \alpha) \text { or } \epsilon-n^{2}=-2 i n^{\prime} \triangle n^{\prime \prime}(1+i \alpha)
$$

$$
\varepsilon+\frac{\omega}{2} \frac{\partial \varepsilon}{\partial \omega}=n^{\prime}\left(n^{\prime}+\omega \frac{d n^{\prime}}{d \omega}\right)=\frac{c}{v_{g}} n^{\prime}
$$


into (E.4)

$$
\begin{aligned}
\frac{2 i \omega}{c^{2}}\left(\varepsilon+\frac{\omega}{2} \frac{\partial \varepsilon}{\partial \omega}\right) \dot{\beta} & =\left(\frac{\omega^{2}}{c^{2}} \varepsilon-k^{2}\right) \beta \\
\frac{2 i \omega}{c^{2}}\left(\frac{c}{v_{g}} n^{\prime}\right) \dot{\beta} & =\left(\frac{\omega^{2}}{c^{2}} \varepsilon-\frac{\omega^{2}}{c^{2}} n^{\prime 2}\right) \beta \\
\frac{2 i \omega}{c v_{g}} n^{\prime} \dot{\beta} & =\frac{\omega^{2}}{c^{2}}\left(\varepsilon-n^{\prime 2}\right) \beta \\
\frac{2 i}{v_{g}} n^{\prime} \dot{\beta} & =\frac{\omega}{c}\left(-2 i n^{\prime} \triangle n^{\prime \prime}(1+i \alpha)\right) \beta \\
\dot{\beta} & =-\frac{\omega}{c} v_{g} \triangle n^{\prime \prime}(1+i \alpha) \beta
\end{aligned}
$$

Then using (E.5)

$$
g-\alpha_{\gamma} \equiv-\frac{2 \omega}{c} n^{\prime \prime}
$$

we replace $n^{\prime \prime}$ with $0+\triangle n^{\prime \prime}$ to get

$$
g-\alpha_{\gamma} \equiv-\frac{2 \omega}{c} \triangle n^{\prime \prime}
$$

or

we get (E.10)

$$
-\frac{\omega}{c} \Delta n^{\prime \prime}=\frac{g-\alpha_{\gamma}}{2}
$$

$$
\dot{\beta}=\frac{\left(g-\alpha_{\gamma}\right) v_{g}}{2}(1+i \alpha) \beta
$$

\section{E.2.8 Derivation of Henry (A15)}

Rewriting (E.15) for convenience

$$
\phi=\frac{1}{2 i} \ln \left(\frac{\beta}{\beta^{*}}\right)
$$

and given $\beta=I^{\frac{1}{2}} e^{i \phi}$ and $\beta^{*}=I^{\frac{1}{2}} e^{-i \phi}$ we have

$$
\begin{aligned}
\frac{\beta}{\beta^{*}} & =\frac{I^{\frac{1}{2}} e^{i \phi}}{I^{\frac{1}{2}} e^{-i \phi}} \\
& =\frac{e^{i \phi}}{e^{-i \phi}} \\
& =e^{2 i \phi} \\
\ln \left(\frac{\beta}{\beta^{*}}\right) & =2 i \phi \\
\phi & =\frac{1}{2 i} \ln \left(\frac{\beta}{\beta^{*}}\right)
\end{aligned}
$$




\section{E.2.9 Calculation of Henry (A16)}

Want to show that (E.16)

$$
\dot{I}=(G-\gamma) I
$$

\section{Use solution to DE}

Given the DE

$$
\dot{\beta}=\left(\frac{G-\gamma}{2}\right)(1+i \alpha) \beta
$$

we know the solution is

$$
\beta=e^{\left(\frac{G-\gamma}{2}\right)(1+i \alpha) t}
$$

and likewise

$$
\beta^{*}=e^{\left(\frac{G-\gamma}{2}\right)(1-i \alpha) t}
$$

SO

$$
\begin{aligned}
I & =\beta \beta^{*} \\
& =e^{\left(\frac{G-\gamma}{2}\right)(1+i \alpha) t} e^{\left(\frac{G-\gamma}{2}\right)(1-i \alpha) t} \\
& =e^{\left\{\left(\frac{G-\gamma}{2}\right)(1-i \alpha) t+\left(\frac{G-\gamma}{2}\right)(1+i \alpha) t\right\}} \\
& =e^{\left\{\left(\frac{G-\gamma}{2}\right)(1-i \alpha+1+i \alpha) t\right\}} \\
& =g e^{\left\{\left(\frac{G-\gamma}{2}\right) 2 t\right\}} \\
& =e^{(G-\gamma) t}
\end{aligned}
$$

And we know the DE for this is

$$
\dot{I}=(G-\gamma) I
$$

Multiply DE

$$
\begin{aligned}
\dot{\beta} \dot{\beta}^{*} & =\left(\frac{G-\gamma}{2}\right)(1+i \alpha) \beta\left(\frac{G-\gamma}{2}\right)(1-i \alpha) \beta^{*} \\
\dot{\beta} \dot{\beta}^{*} & =\left(\frac{G-\gamma}{2}\right)^{2}\left(1+\alpha^{2}\right) \beta \beta^{*} \\
\dot{I} & =\left(\frac{G-\gamma}{2}\right)^{2}\left(1+\alpha^{2}\right) I
\end{aligned}
$$

This does not agree with Henry! 


\section{E.2.10 Calculation of Henry (A17)}

Want to get to (E.17)

$$
\dot{\phi}=\frac{\alpha}{2}(G-\gamma)
$$

Given

$$
\beta=I^{\frac{1}{2}} e^{i \phi}
$$

and

$$
\beta=e^{\left(\frac{G-\gamma}{2}\right)(1+i \alpha) t}
$$

we have

$$
\begin{aligned}
\phi & =\frac{1}{2 i} \ln \left(\frac{\beta}{\beta^{*}}\right) \\
& =\frac{1}{2 i} \ln \left(\frac{e^{\left(\frac{G-\gamma}{2}\right)(1+i \alpha) t}}{e^{\left(\frac{G-\gamma}{2}\right)(1-i \alpha) t}}\right) \\
& =\frac{1}{2 i} \ln \left(e^{\left(\frac{G-\gamma}{2}\right)(1+i \alpha-1+i \alpha) t}\right) \\
& =\frac{1}{2 i} \ln \left(e^{(G-\gamma) i \alpha t}\right) \\
& =\frac{1}{2 i}(G-\gamma) i \alpha t \\
& =\frac{\alpha}{2}(G-\gamma) t
\end{aligned}
$$

Therefore

$$
\dot{\phi}=\frac{\alpha}{2}(G-\gamma)
$$

\section{E.2.11 Net Gain or Loss and the Complex Index of Refraction}

Want to calculate the net gain or loss through a medium. Using the attenuation (or gain) coefficient [KasapOp2013, (1.8.6)]

$$
\alpha_{a t t}=2 k^{\prime \prime}
$$

where

$$
\begin{aligned}
k & =\frac{n \omega}{c} \\
k^{\prime}-i k^{\prime \prime} & =\frac{\omega}{c}\left(n^{\prime}-i n^{\prime \prime}\right)
\end{aligned}
$$

Therefore

$$
k^{\prime \prime}=\frac{\omega}{c} n^{\prime \prime}
$$

and

$$
\alpha_{a t t}=2 k^{\prime \prime}=\frac{2 \omega}{c} n^{\prime \prime}
$$


Net gain or loss of system versus material

$$
\begin{gathered}
E=A e^{i(\omega t-k z)}=A e^{i\left(\omega t-\left[k^{\prime}-i k^{\prime \prime}\right] z\right)}=A e^{i\left(\omega t-k^{\prime} z+i k^{\prime \prime} z\right)}=A e^{-k^{\prime \prime} z} e^{i\left(\omega t-k^{\prime} z\right)} \\
k=\frac{2 \pi}{\lambda}\left(n^{\prime}-i n^{\prime \prime}\right) \\
E=A e^{-\frac{2 \pi}{\lambda} n^{\prime \prime} z} e^{i\left[\omega t-\frac{2 \pi}{\lambda} n^{\prime} z\right]}
\end{gathered}
$$




\section{Appendix F}

\section{Henry Factor}

\section{F.1 Derivation of Henry Factor}

This derivation follows along [20]. To explore the Henry factor $\left(\alpha_{H}\right)$ we will examine how a light wave propagates through the active layer. The electric field will have phase $(\beta)$ and gain $(g)$.

$$
E(z)=E_{0} e^{-j \beta z} e^{g z}
$$

where of course $\beta$ and $g$ have units $\left[\frac{1}{\text { Length }}\right]$. The real and imaginary parts of the complex relative permittivity are linked to the real and imaginary parts of the complex refractive index.

$$
\beta+j g=\omega \sqrt{\left(\varepsilon_{r r}+j \varepsilon_{r i}\right) \varepsilon_{0} \mu_{0}}=\frac{\omega}{c} \sqrt{\varepsilon_{r r}+j \varepsilon_{r i}}=\frac{\omega}{c}\left(n_{r}+j n_{i}\right)
$$

Note also

$$
\beta+j g=k_{r}+j k_{i}
$$

and

$$
\sqrt{\varepsilon_{r r}+j \varepsilon_{r i}}=n_{r}+j n_{i}
$$

and

$$
\varepsilon_{r r}+j \varepsilon_{r i}=\left(n_{r}^{2}-n_{i}^{2}\right)+j\left(2 n_{r} n_{i}\right)
$$

Note: Carroll doesn't use the convention of negating the imaginary components!

The 'gain response' is uniquely defined by the 'phase response' so that $\beta$ and $g$ are linked and consequently the real part of the complex refractive index $n_{r}$ is linked to the imaginary part of the refractive index $n_{i}$. The processes of calculating the real response from the imaginary, and vice versa, are known as Hilbert transforms or Kramers-Krönig transforms. This is not a direct transformation and a change in gain at a particular frequency does not necessarily result in a refractive-index change at the same frequency. 


\section{F.2 Henry Factor Example}

$\alpha_{H}$ is calculated as change in real part of $n$ over the imaginary part of $n$ as $N$ is changed. This is assumed to be from $N_{t r}$, the carrier density at transparency.

$$
\begin{aligned}
& \alpha_{H}=-\frac{\frac{d n_{r}}{d N}}{\frac{d n_{i}}{d N}} \\
& \alpha_{H} \simeq-\frac{\triangle n_{r}}{\triangle n_{i}}
\end{aligned}
$$

If we take a sample index of refraction we can see how $\alpha_{H}$ will vary with frequency. This was shown in figure 2.23.

Now

$$
k_{r}=\frac{\omega_{f s}}{c} n_{r}=\frac{2 \pi f_{s}}{c} n_{r}=\frac{2 \pi f_{s}}{v_{p h}}=\frac{2 \pi}{\lambda_{f s}}
$$

SO

$$
\frac{\omega_{f s}}{c}=\frac{2 \pi}{\lambda_{f s} n_{r}}
$$

and

$$
g\left(=k_{i}\right)=\frac{\omega}{c} n_{i}=\frac{2 \pi}{\lambda_{f s}} n_{i}
$$

so that

$$
\triangle g=\frac{2 \pi}{\lambda_{f s}} \triangle n_{i}
$$

or rearranging

$$
\triangle n_{i}=\frac{\lambda_{f s}}{2 \pi} \triangle g
$$

and substituting back into (F.6) we get

$$
\alpha_{H} \simeq-\frac{\triangle n_{r}}{\triangle n_{i}}=-\frac{2 \pi \triangle n_{r}}{\lambda_{f s} \triangle g}
$$

Solving for $\triangle n_{r}$

$$
\triangle n_{r}=-\alpha_{H} \frac{\lambda_{f s}}{2 \pi} \triangle g
$$

\section{F.3 Chirp}

Laser frequency is controlled by the optical length. Changing the gain will change the carrier density $(N)$ and consequently the index. This means the change in frequency (chirp) is proportional to the change in gain $\triangle g$. In section F.4 we derive

$$
\frac{\triangle f}{f} \simeq-\frac{\triangle n}{n}
$$




$$
\frac{\triangle f_{g}}{f} \sim-\frac{\overline{\triangle n_{r}}}{n_{r}} \sim \frac{\lambda_{f s}}{2 \pi} \overline{\alpha_{H} \triangle g}
$$

Using (F.13)

$$
\frac{\triangle f_{g}}{f} \sim-\frac{\overline{\triangle n_{r}}}{n_{r}} \sim \frac{\lambda_{f s}}{2 \pi} \frac{\overline{\alpha_{H} \triangle g}}{n_{r}}
$$

F.4 Change in laser frequency due to change in real index of refraction

$$
f=\frac{v}{\lambda}=\frac{c}{n \lambda}
$$

and

$$
\begin{aligned}
\triangle f & =\frac{c}{(n+\triangle n) \lambda}-\frac{c}{n \lambda} \\
& =\frac{c}{\lambda}\left[\frac{1}{n+\triangle n}-\frac{1}{n}\right] \\
& =\frac{c}{\lambda}\left[\frac{n-(n+\triangle n)}{(n+\triangle n) n}\right] \\
& =-\frac{c}{n \lambda}\left[\frac{\triangle n}{n+\triangle n}\right] \\
& =-f\left[\frac{\triangle n}{n+\triangle n}\right]
\end{aligned}
$$

If we assume $\Delta n \ll n$ then

$$
\frac{\triangle f}{f} \simeq-\frac{\triangle n}{n}
$$




\section{Appendix G}

\section{Derivation of TWM from Maxwell's Equations}

If we start with the 2nd-order wave equation (from Maxwell's equations) we can show that use of the SVEA reduces the 2nd-order wave equation to a 1st-order wave equation. This is what we call the traveling wave equation. There are actually two of them-one for each direction.

\section{G.1 Wave Traveling Right}

Because we design our wave guide to support transverse waves we can assume a plane wave traveling in the direction of our wave guide, which we define as $z . E_{+}$in (G.1) is the complex envelope $E_{+}$and the high frequency complex exponential carrier, both of which travel to the right.

$$
E(z, t)=E_{+}(z, t) e^{i\left(\omega_{0} t-k_{0} z\right)}
$$

Let us substitute this into 1-D 2nd-order wave equation and evaluate.

$$
\begin{gathered}
\frac{\partial^{2} E}{\partial z^{2}}=\frac{1}{v^{2}} \frac{\partial^{2} E}{\partial t^{2}} \\
\frac{\partial^{2}}{\partial z^{2}}\left(E_{+}(z, t) e^{i\left(\omega_{0} t-k_{0} z\right)}\right)=\frac{1}{v^{2}} \frac{\partial^{2}}{\partial t^{2}}\left(E_{+}(z, t) e^{i\left(\omega_{0} t-k_{0} z\right)}\right) \\
\frac{\partial}{\partial z}\left\{\left[\frac{\partial E_{+}}{\partial z}+E_{+}\left(-i k_{0}\right)\right] e^{i\left(\omega_{0} t-k_{0} z\right)}\right\}=\frac{1}{v^{2}} \frac{\partial}{\partial t}\left\{\left[\frac{\partial E_{+}}{\partial t}+E_{+}\left(i \omega_{0}\right)\right] e^{i\left(\omega_{0} t-k_{0} z\right)}\right\} \\
{\left[\frac{\partial^{2} E_{+}}{\partial z^{2}}-i k_{0} \frac{\partial E_{+}}{\partial z}+\left(\frac{\partial E_{+}}{\partial z}-i k_{0} E_{+}\right)\left(-i k_{0}\right)\right] e^{i\left(\omega_{0} t-k_{0} z\right)}=} \\
\frac{1}{v^{2}}\left[\frac{\partial^{2} E_{+}}{\partial t^{2}}+i \omega_{0} \frac{\partial E_{+}}{\partial t}+\left(\frac{\partial E_{+}}{\partial t}+i \omega_{0} E_{+}\right)\left(i \omega_{0}\right)\right] e^{i\left(\omega_{0} t-k_{0} z\right)}
\end{gathered}
$$


Cancel high frequency carrier on both sides and simplify

$$
\frac{\partial^{2} E_{+}}{\partial z^{2}}-2 i k_{0} \frac{\partial E_{+}}{\partial z}-k_{0}^{2} E_{+}=\frac{1}{v^{2}}\left\{\frac{\partial^{2} E_{+}}{\partial t^{2}}+2 i \omega_{0} \frac{\partial E_{+}}{\partial t}-\omega_{0}^{2} E_{+}\right\}
$$

Given that $E_{+}$is slowly varying we can neglect the second derivatives, which results in a first-order wave equation:

$$
\begin{aligned}
-2 i k_{0} \frac{\partial E_{+}}{\partial z}-k_{0}^{2} E_{+} & =\frac{1}{v^{2}}\left\{2 i \omega_{0} \frac{\partial E_{+}}{\partial t}-\omega_{0}^{2} E_{+}\right\} \\
\left(\frac{1}{v^{2}} 2 i \omega_{0} \frac{\partial}{\partial t}+2 i k_{0} \frac{\partial}{\partial z}\right) E_{+} & =\left(\frac{1}{v^{2}} \omega_{0}^{2}-k_{0}^{2}\right) E_{+}
\end{aligned}
$$

Noting that the phase velocity of the wave $v=\frac{\omega_{0}}{k_{0}}$

$$
\begin{aligned}
\left(\frac{1}{v^{2}} 2 i \omega_{0} \frac{\partial}{\partial t}+2 i \frac{\omega_{0}}{v} \frac{\partial}{\partial z}\right) E_{+} & =\left(\frac{k_{0}^{2}}{\omega_{0}^{2}} \omega_{0}^{2}-k_{0}^{2}\right) E_{+} \\
\left(\frac{1}{v^{2}} 2 i \omega_{0} \frac{\partial}{\partial t}+\frac{1}{v} 2 i \omega_{0} \frac{\partial}{\partial z}\right) E_{+} & =0 \\
\left(\frac{\partial}{\partial t}+v \frac{\partial}{\partial z}\right) E_{+} & =0
\end{aligned}
$$

This means that the carrier with a slowly varying amplitude will be a solution to the 2nd-order wave equation if $E_{+}$is a solution to the 1st-order wave equation. But $v=\frac{\omega_{0}}{k_{0}}$ is the phase velocity not the group velocity! The requirement is that $v_{g}=v_{p h}=v$.

We could just use the 2nd-order 1-D wave equation but this would require a much smaller time step and therefore many more steps for any given simulation. This is extremely costly. By using the slowly varying amplitude approximation, we increase the time step and may completely ignore the high frequency carrier.

\section{G.2 Wave Traveling Left}

We can do the exact same analysis with a left-traveling wave to get the $E_{-}$first-order wave equation.

$$
\begin{gathered}
E(z, t)=E_{-}(z, t) e^{i\left(\omega_{0} t+k_{0} z\right)} \\
\frac{\partial^{2}}{\partial z^{2}}\left(E_{-}(z, t) e^{i\left(\omega_{0} t+k_{0} z\right)}\right)=\frac{1}{v^{2}} \frac{\partial^{2}}{\partial t^{2}}\left(E_{-}(z, t) e^{i\left(\omega_{0} t+k_{0} z\right)}\right) \\
\frac{\partial}{\partial z}\left\{\left[\frac{\partial E_{-}}{\partial z}+E_{-}\left(i k_{0}\right)\right] e^{i\left(\omega_{0} t+k_{0} z\right)}\right\}=\frac{1}{v^{2}} \frac{\partial}{\partial t}\left\{\left[\frac{\partial E_{-}}{\partial t}+E_{-}\left(i \omega_{0}\right)\right] e^{i\left(\omega_{0} t+k_{0} z\right)}\right\}
\end{gathered}
$$




$$
\begin{aligned}
{\left[\frac{\partial^{2} E_{-}}{\partial z^{2}}+i k_{0} \frac{\partial E_{-}}{\partial z}+\left(\frac{\partial E_{-}}{\partial z}\right.\right.} & \left.\left.+i k_{0} E_{+}\right)\left(i k_{0}\right)\right] e^{i\left(\omega_{0} t-k_{0} z\right)} \\
& =\frac{1}{v^{2}}\left[\frac{\partial^{2} E_{-}}{\partial t^{2}}+i \omega_{0} \frac{\partial E_{-}}{\partial t}+\left(\frac{\partial E_{-}}{\partial t}+i \omega_{0} E_{-}\right)\left(i \omega_{0}\right)\right] e^{i\left(\omega_{0} t-k_{0} z\right)}
\end{aligned}
$$

Cancel high frequency carrier on both sides and simplify

$$
\frac{\partial^{2} E_{-}}{\partial z^{2}}+2 i k_{0} \frac{\partial E_{-}}{\partial z}-k_{0}^{2} E_{-}=\frac{1}{v^{2}}\left\{\frac{\partial^{2} E_{-}}{\partial t^{2}}+2 i \omega_{0} \frac{\partial E_{-}}{\partial t}-\omega_{0}^{2} E_{-}\right\}
$$

Given that $E_{-}$is slowly varying we can neglect the second derivatives, which results in a first-order wave equation:

$$
\begin{aligned}
2 i k_{0} \frac{\partial E_{-}}{\partial z}-k_{0}^{2} E_{-} & =\frac{1}{v^{2}}\left\{2 i \omega_{0} \frac{\partial E_{-}}{\partial t}-\omega_{0}^{2} E_{-}\right\} \\
\left(\frac{1}{v^{2}} 2 i \omega_{0} \frac{\partial}{\partial t}-2 i k_{0} \frac{\partial}{\partial z}\right) E_{-} & =\left(\frac{1}{v^{2}} \omega_{0}^{2}-k_{0}^{2}\right) E_{-}
\end{aligned}
$$

Noting that the phase velocity of the wave $v=\frac{\omega_{0}}{k_{0}}$

$$
\begin{aligned}
\left(\frac{1}{v^{2}} 2 i \omega_{0} \frac{\partial}{\partial t}-2 i \frac{\omega_{0}}{v} \frac{\partial}{\partial z}\right) E_{-} & =0 \\
\left(\frac{1}{v} \frac{\partial}{\partial t}-\frac{\partial}{\partial z}\right) E_{-} & =0 \\
\left(\frac{\partial}{\partial t}-v \frac{\partial}{\partial z}\right) E_{-} & =0
\end{aligned}
$$

\section{G.3 Group Velocity}

The SVEA simplifies the model significantly with the requirement that the phase velocity of the envelope equal the phase velocity of the carrier. Since we ignore the carrier we will label the envelope phase velocity as the total field group velocity. sion.

Because the group velocity is constant (and equal the phase velocity) there is no disper- 


\section{Appendix $\mathbf{H}$}

\section{Derivation of Rate Equations for REM}

Following along in [20] we first develop a form of the classic rate equations and then a simplified version of the traveling wave equations.

\section{H.1 Classic Rate Equations}

Assume an ideal laser made from a waveguide with a uniform density of photons in the active layer interacting with a uniform density of electrons. The rate of change of photons is given by

$$
\begin{aligned}
\frac{d(V S)}{d t} & =\Gamma V G_{m}(N) S-V A_{m} S-\frac{V S}{\tau_{p}}+\beta_{s p} \Gamma \frac{V N}{\tau_{s p}} \\
\frac{d(S)}{d t} & =\Gamma G_{m}(N) S-A_{m} S-\frac{S}{\tau_{p}}+\beta_{s p} \Gamma \frac{N}{\tau_{s p}}
\end{aligned}
$$

where $V$ is the volume of the cavity. The photon lifetime $\tau_{p}$ is an estimate of the rate of escape of photons out of the cavity while the term $\tau_{s p}$ gives the spontaneous emission caused by electrons recombining with holes, giving out a random emission of light over a range of frequencies in all directions. The coupling factor $\beta_{s p}$ accounts for the fact that only a (typically small) portion of this will couple to the lasing mode. This spontaneous emission is insignificant once the device is lasing but is essential around the lasing threshold.

The gain term $G_{m}$ is the material optical power gain per unit time. The material optical absorption per unit time is $A_{m}$. Calculation of these terms is shown H.1.1.

(H.1) agrees with the photon equation of the REM but has the extra loss term $A_{m} S$. Since this is losses outside of the active region it is not counted in the REM. The equation is just counting particles (photons) and using the principle of detailed balance. We then cancel out the volume. 
If we apply the same principle to the rate of change of carriers we get a similar expression.

$$
\begin{aligned}
\frac{d(\Gamma V N)}{d t} & =-\Gamma V G_{m}(N) S-\frac{\Gamma V N}{\tau_{r}}+\frac{I}{q} \\
\frac{d(\Gamma N)}{d t} & =-\Gamma G_{m}(N) S-\frac{\Gamma N}{\tau_{r}}+\frac{I}{q V}
\end{aligned}
$$

where $\Gamma$ is the confinement factor discussed in section 2.1.4. The first term is identical to the first term of the photon equation (H.1) but with opposite sign. This is because again we are just counting the particles (electrons) that are now moving to the lower energy state due to stimulated emission. From the point of view of these equations the electrons are being removed while the photons are being generated. Again (H.2) agrees with the electron equation of the REM.

A quick look at the units shows from the photon equation

$$
\frac{V\left[L^{3}\right] S\left[\frac{\text { photon }}{L^{3}}\right]}{t} \equiv\left[\frac{\text { photon }}{t}\right]
$$

and the second term of the photon equation

$$
\frac{\Gamma[] V\left[L^{3}\right] G_{m}\left[t^{-1}\right]}{t} S\left[\frac{\text { photon }}{L^{3}}\right] \equiv\left[\frac{\text { photon }}{t}\right]
$$

and the carrier equation

$$
\Gamma[] V\left[L^{3}\right] N\left[\frac{\text { electron }}{L^{3}}\right] \equiv\left[\frac{\text { electron }}{t}\right]
$$

These show that we are just watching the number of particles grow or shrink -in the normal form of the equations (such as in REM) it is the increase and decrease of concentrations.

Note that the confinement factor $\Gamma$ does not occur in the photon-only terms. This is because the volume $(\Gamma V)$ where electron-hole recombination is smaller than the volume $(V)$ occupied by the photons. Refer to figure 3.1.

In this simplified analysis it was assumed that $N \sim P$ which is normally the case. If these charge carriers did not neutralize each other their high densities would produce strong fields that would affect the charge carriers. This would be on a short enough timescale to require a more complicated rate equation.

\section{H.1.1 Optical Power Gain and Loss Parmameters}

The material power gain is

$$
G_{m}(N)=G_{m}^{\prime}\left(N-N_{t r}\right) G(N)=G^{\prime}\left(N-N_{t r}\right)
$$

Net power gain per unit time

$$
G=\Gamma G_{m}(N)-A_{m}
$$


A lower case $g_{m}$ is used for the field gain or loss per unit distance and is a function of $N$. As with the power gain above we have a differential field gain

$$
g_{m}(N)=g_{m}^{\prime}\left(N-N_{t r}\right)
$$

The net field gain including confinement factor and loss

$$
g=\Gamma g_{m}=a_{0}
$$

There is a link between the power gain/loss per unit time and field gain/loss per unit distance.

$$
\begin{aligned}
G & =2 g v_{g} \\
G_{m} & =2 g_{m} v_{g}
\end{aligned}
$$

where $v_{g}$ is the group or energy velocity. The factor of 2 arises because $S \propto E^{2}$.

$$
\frac{d S}{d t}=G S=v_{g} \frac{d S}{d z}=2 v_{g} E \frac{d E}{d z}=2 v_{g} g E^{2}
$$




\section{Appendix I}

\section{Derivation of REM from TWM}

For this derivation $\kappa$ is used where normally $g_{p}$ would be. This is the Lorentzian gain parameter in the TWM.

\section{I.1 Carrier equation}

Since the TWM uses the classic REM $N$ equation for the carrier density for each slice of the active layer, it will be the same form only now it will be a function of $z$. The time dependence of $N$ is explicitly shown to remind us that it is changing with time and so the derivative with respect to time makes sense.

$$
\frac{d N(z, t)}{d t}=\frac{I}{q V}-g_{0}\left(N(z, t)-N_{t r}\right) S-\frac{N(z, t)}{\tau_{n}}
$$

The average is calculated by integrating over the length and then dividing by the length.

$$
\begin{aligned}
\frac{d \bar{N}(t)}{d t} & =\frac{1}{L} \int_{0}^{L} \frac{d N(z, t)}{d t} d z \\
& =\frac{1}{L} \int_{0}^{L}\left\{\frac{I}{q V}-g_{0}\left(N(z, t)-N_{t r}\right) S-\frac{N(z, t)}{\tau_{n}}\right\} d z \\
& =\frac{I}{q V}+g_{0}\left(\bar{N}(t)-N_{t r}\right) S-\frac{\bar{N}(t)}{\tau_{n}}
\end{aligned}
$$

where

$$
\begin{aligned}
& \bar{N}(t)=\frac{1}{L} \int_{0}^{L} N(z, t) d z \\
& \bar{S}(t)=\frac{1}{L} \int_{0}^{L} S(z, t) d z
\end{aligned}
$$

and we've assumed

$$
\bar{N}(t) \bar{S}(t) \simeq \frac{1}{L} \int_{0}^{L} N(z, t) S(z, t) d z
$$


Even though this approximation will never be exact in the typical case with the SVEA the variables are essentially constant and it will be a good one.

\section{I.2 Photon equation}

\section{I.2.1 Forward Traveling Wave and Material Response}

Start with the simple traveling wave equation (in the forward direction) (5.1)

$$
\left(\frac{\partial}{\partial t}+v \frac{\partial}{\partial z}\right) E(z, t)=-i v \beta E(z, t)
$$

Substituting in for $\beta$ from (5.2) but using only the gain and Henry factor terms

$$
\begin{aligned}
& \left(\frac{\partial}{\partial t}+v \frac{\partial}{\partial z}\right) E(z, t)=-i\left\{\frac{1}{2}\left(i+\alpha_{H}\right) g\right\} v E(z, t) \\
& \left(\frac{\partial}{\partial t}+v \frac{\partial}{\partial z}\right) E(z, t)=\frac{g}{2}\left(1-i \alpha_{H}\right) v E(z, t)
\end{aligned}
$$

Replace the gain parameter $g$ with the stimulated emission gain term $g_{0}\left(N-N_{t r}\right)$ from (3.9) and add the polarization term from (5.46) and spontaneous emission as a nonlinear function $F_{s}$.

$$
\left(\frac{\partial}{\partial t}+v \frac{\partial}{\partial z}\right) E(z, t)=g_{0} \frac{N-N_{t r}}{2}\left(1-i \alpha_{H}\right) v E(z, t)-g_{p}(E(z, t)-P)+F_{s}
$$

or

$$
\begin{aligned}
\left(\frac{\partial}{\partial t}+v \frac{\partial}{\partial z}\right) E(z, t)=g_{0} \frac{N-N_{t r}}{2} v E(z, t)-i \alpha_{H} g_{0} \frac{N-N_{t r}}{2} & v E(z, t) \\
& -g_{p}(E(z, t)-P)+F_{s}
\end{aligned}
$$

Assuming a linear response and knowing that $P$ is a function of $\omega$ we have

$$
P(\omega)=\Gamma(\omega) E(\omega)
$$

and so we will have a $\Gamma$ for each mode of the laser

\section{I.2.2 Forward Traveling Mode}

$$
P\left(\omega_{j}\right)=\Gamma_{j}(\omega) E\left(\omega_{j}\right)
$$

If we define the field component for the $j$ 'th mode $E\left(\omega_{j}\right)=E_{j}$, and $P\left(\omega_{j}\right)=P_{j}$ then

$$
P_{j}=\Gamma_{j} E_{j}
$$


and use this in (I.7) for each mode

$$
\begin{aligned}
\left(\frac{\partial}{\partial t}+v \frac{\partial}{\partial z}\right) E_{j} & =g_{0} \frac{N-N_{t r}}{2}\left(1-i \alpha_{H}\right) v E_{j}-\kappa\left(E_{j}-P_{j}\right)+F_{s j} \\
& \left.=g_{0} \frac{N-N_{t r}}{2}\left(1-i \alpha_{H}\right) v E_{j}-\kappa\left(1-\Gamma_{j}\right)\right) E_{j}+F_{s j} \\
& =g_{0} \frac{N-N_{t r}}{2}\left(1-i \alpha_{H}\right) v E_{j}-K_{j} E_{j}+F_{s j}
\end{aligned}
$$

where

$$
K_{j}=\kappa\left(1-\Gamma_{j}\right)
$$

Separating real and imaginary we get

$$
\left(\frac{\partial}{\partial t}+v \frac{\partial}{\partial z}\right) E_{j}=\left\{g_{0} \frac{N-N_{t r}}{2}-\frac{\Re\left[K_{j}\right]}{v}\right\} v E_{j}-i\left\{g_{0} \frac{N-N_{t r}}{2} \alpha_{H}+\frac{\Im\left[K_{j}\right]}{v}\right\} v E_{j}+F_{s j}
$$

We note from sections 5.3 and 5.4 that a real $\beta$ is associated with a rotation while an imaginary $\beta$ is associated with a gain or loss. The form of the equations (5.8) and (5.15) has $\beta$ multiplied by ' $i$ '. This tells us that the imaginary part on the right-hand side is associated with a rotation while the real part is associated with a gain. This is why, unlike with $\beta$, the real part of of the Lorentzian $\left(K_{j}\right)$ is associated with the gain and the imaginary the rotation.

$$
\begin{aligned}
& g_{j}=\frac{g_{0}}{2}\left(N-N_{t r}\right)-\frac{\Re\left(K_{j}\right)}{v} \\
& h_{j}=\alpha_{h} \frac{g_{0}}{2}\left(N-N_{t r}\right)+\frac{\Im\left(K_{j}\right)}{v}
\end{aligned}
$$

This now makes $g_{j}$ the gain term and $h_{j}$ the rotation term

$$
\begin{aligned}
\left(\frac{\partial}{\partial t}+v \frac{\partial}{\partial z}\right) E_{j} & =\left(g_{j}-i h_{j}\right) v E_{j}+F_{s j} \\
\left(\frac{\partial}{\partial t}+v \frac{\partial}{\partial z}\right) E_{j} & =-i v\left(i g_{j}+h_{j}\right) E_{j}+F_{s j} \\
\beta_{j} & =h_{j}+i g_{i}
\end{aligned}
$$

so

$$
\left(\frac{\partial}{\partial t}+v \frac{\partial}{\partial z}\right) E_{j}=-i v \beta_{j} E_{j}+F_{s j}
$$


Starting with

$$
\Gamma(\omega)=\frac{\gamma_{p}}{\gamma_{p}+i\left(\omega-\omega_{0}\right)}
$$

For the fundamental laser mode $\left(\omega_{1}=0\right)$ assuming the frequency response of the gain is centered at this mode $\left(\omega_{0}=0\right)$, we have

$$
\begin{aligned}
\Gamma_{1} & =\frac{\gamma_{p}}{\gamma_{p}+i\left(\omega_{1}-\omega_{0}\right)} \\
& =\frac{\gamma_{p}}{\gamma_{p}+i(0-0)} \\
& =1
\end{aligned}
$$

$K_{1}$ is $\kappa\left(1-\Gamma_{1}\right)=0$ so the nominal gain is $g_{1}=\frac{g_{0}}{2}\left(N-N_{t r}\right)-\Re\left(K_{1}\right)=\frac{g_{0}}{2}\left(N-N_{t r}\right)$ which means the polarization term has no effect which is as expected at the fundamental mode.

For the higher modes we have

$$
\Gamma_{j}=\frac{\gamma_{p}}{\gamma_{p}+i\left(\omega_{j}-\omega_{0}\right)}
$$

and $K_{j}=\kappa\left(1-\Gamma_{j}\right)$, a complex number. The nominal gain is $g_{j}=\frac{g_{0}}{2}\left(N-N_{t r}\right)-\Re\left(K_{j}\right)$.

As $\omega$ goes to infinity we get

$$
\begin{aligned}
\Gamma_{\infty} & =\frac{\gamma_{p}}{\gamma_{p}+i\left(\omega_{\infty}-\omega_{0}\right)} \\
& =0
\end{aligned}
$$

and $K_{\infty}=\kappa\left(1-\Gamma_{\infty}\right)=\kappa$ so the nominal gain is $g_{\infty}=\frac{g_{0}}{2}\left(N-N_{t r}\right)-\kappa=0$ and therefore we can conclude

$$
\kappa=\frac{g_{0}}{2}\left(N-N_{t r}\right)
$$

Note that $g_{0}$ is used in both the REM and TWM while $g_{p}$ (sometimes referred to as $\kappa$ ) is in the TWM only. This is a required condition to derive REM from TWM and is a problem because the condition in (I.13) is not always true. .

\section{I.2.3 Forward Traveling Photon Mode Density}

Let the forward traveling photon density be

$$
S_{j}=E_{j} E_{j}^{*}
$$

It's derivative is therefore

$$
\frac{d S_{j}}{d z}=\frac{d E_{j}}{d z} E_{j}^{*}+\frac{d E_{j}^{*}}{d z} E_{j}
$$


The propagation equation and conjugate must both hold:

$$
\begin{aligned}
& \frac{\partial E_{j}}{\partial t}+v \frac{\partial E_{j}}{\partial z}=-i \beta_{i} E_{j}+F_{s j} \\
& \frac{\partial E_{j}^{*}}{\partial t}+v \frac{\partial E_{j}^{*}}{\partial z}=i \beta_{j}^{*} E_{j}^{*}+F_{s j}^{*}
\end{aligned}
$$

Multiply the propagation equation by the conjugate of the field and conjugate equation by the field:

$$
\begin{aligned}
& \frac{\partial E_{j}}{\partial t} E_{j}^{*}+v \frac{\partial E_{j}}{\partial z} E_{j}^{*}=-i \beta_{j} E_{j} E_{j}^{*}+F_{s j} E_{j}^{*} \\
& \frac{\partial E_{j}^{*}}{\partial t} E_{j}+v \frac{\partial E_{j}^{*}}{\partial z} E_{j}=i \beta_{i}^{*} E_{j}^{*} E_{j}+F_{s j}^{*} E_{j}
\end{aligned}
$$

Add the two equations

$$
\frac{\partial E_{j}}{\partial t} E_{j}^{*}+\frac{\partial E_{j}^{*}}{\partial t} E_{j}+v \frac{\partial E_{j}}{\partial z} E_{j}^{*}+v \frac{\partial E_{j}^{*}}{\partial z} E_{j}=i\left(\beta_{j}^{*}-\beta_{j}\right) E_{j} E_{j}^{*}+F_{s j} E_{j}^{*}+F_{s j}^{*} E_{j}
$$

Use definition and general derivative of $S_{j}$ eliminate $E_{j}$ :

$$
\begin{array}{r}
\frac{\partial S_{j}}{\partial t}+v \frac{\partial S_{j}}{\partial z}=i\left(\beta_{j}^{*}-\beta_{j}\right) S_{j}+F_{s j} E_{j}^{*}+F_{s j}^{*} E_{j} \\
\frac{\partial S_{j}}{\partial t}+v \frac{\partial S_{j}}{\partial z}=2 \Im\left(\beta_{j}\right) S_{j}+F_{s j} E_{j}^{*}+F_{s j}^{*} E_{j}
\end{array}
$$

Need to define a spontaneous emission term

$$
S_{p j}=F_{s j}^{*} E_{j}+F_{s j} E_{j}^{*}
$$

Giving a traveling wave equation for the forward traveling photons:

$$
\frac{\partial S_{f i}}{\partial t}+v \frac{\partial S_{f i}}{\partial z}=2 \Im\left(\beta_{i}\right) S_{f i}+S_{p f i}
$$

Now we integrate the equation over $z$ and obtain average values. First define:

$$
\begin{aligned}
\bar{S}_{j} & =\frac{1}{L} \int_{0}^{L} S_{j} d z \\
\bar{G}_{j} & =\frac{1}{L} \int_{0}^{L} g_{j} d z=\Im\left(\beta_{j}\right) \\
\bar{G}_{j} \bar{S}_{j} & \approx \frac{1}{L} \int_{0}^{L} 2 \Im\left(\beta_{j}\right) S_{j} d z=\frac{1}{L} \int_{0}^{L} 2 g_{j} S_{j} d z
\end{aligned}
$$


If we assume $\frac{\partial S_{j}}{\partial t}$ is approximately constant at some average value, then integrating the equation over the length gives:

$$
\begin{array}{r}
\frac{1}{L} \int_{0}^{L}\left\{\frac{\partial S_{j}}{\partial t}+v \frac{\partial S_{j}}{\partial z}=2 \Im\left(\beta_{j}\right) S_{j}+S_{p j}\right\} d z \\
\frac{1}{L} \frac{\partial \bar{S}_{j}}{\partial t} \int_{0}^{L} d z+\frac{v}{L} \int_{0}^{L} \frac{\partial S_{j}}{\partial z} d z=\frac{1}{L} \int_{0}^{L} 2 \Im\left(\beta_{j}\right) S_{j} d z+L S_{p j} \\
\frac{\partial \bar{S}_{j}}{\partial t}+\frac{v}{L}\left[S_{j}(L)-S_{j}(0)\right]=\bar{G}_{j} \bar{S}_{j}+S_{p j}
\end{array}
$$

So far we have only been looking at the forward traveling field equation. There is also a reverse traveling companion. We will add some notation to now show both using $f$ for forward and $r$ for reverse directions. Note the sign change for the reverse traveling wave.

$$
\begin{aligned}
\frac{\partial \bar{S}_{f j}}{\partial t}+\frac{v}{L}\left[S_{f j}(L)-S_{f j}(0)\right] & =\bar{G}_{j} \bar{S}_{f j}+\bar{S}_{p f j} \\
\frac{\partial \bar{S}_{r j}}{\partial t}-\frac{v}{L}\left[S_{r j}(L)-S_{r j}(0)\right] & =\bar{G}_{j} \bar{S}_{r j}+\bar{S}_{p r j}
\end{aligned}
$$

Adding the two equations together and defining a total photon density and total spontaneous photon creation term:

$$
\begin{aligned}
\bar{S}_{j} & =\bar{S}_{f j}+\bar{S}_{r j} \\
\bar{S}_{p j} & =\bar{S}_{p f j}+\bar{S}_{p r j}
\end{aligned}
$$

we get:

$$
\frac{\partial \bar{S}_{j}}{\partial t}+\frac{v}{L}\left[S_{f j}(L)-S_{f j}(0)-S_{r j}(L)+S_{r j}(0)\right]=\bar{G}_{j} \bar{S}_{j}+\bar{S}_{p j}
$$

Assuming a symmetry for forward and reverse photon distributions we have

$$
S_{f j}(0)=S_{r j}(L) \text { and } S_{r j}(0)=S_{f j}(L)
$$

and we can rewrite the equation to be in terms of photon densities at one end $(z=L)$.

$$
\begin{aligned}
\frac{\partial \bar{S}_{j}}{\partial t}+\frac{v}{L}\left[S_{f j}(L)-S_{f j}(0)-S_{r j}(L)+S_{r j}(0)\right] & =\bar{G}_{j} \bar{S}_{j}+\bar{S}_{p j} \\
\frac{\partial \bar{S}_{j}}{\partial t}+\frac{v}{L}\left[S_{f j}(L)-S_{r j}(L)-S_{r j}(L)+S_{f j}(L)\right] & =\bar{G}_{j} \bar{S}_{j}+\bar{S}_{p j} \\
\frac{\partial \bar{S}_{j}}{\partial t}+\frac{2 v}{L}\left[S_{f j}(L)-S_{r j}(L)\right] & =\bar{G}_{j} \bar{S}_{j}+\bar{S}_{p j}
\end{aligned}
$$


Starting with $S_{j}$ at one end $(z=L)$ we have

$$
S_{j}(L)=S_{f j}(L)+S_{r j}(L)
$$

and given that the reflectivity is $R$ we also have

$$
S_{r j}(L)=R S_{f j}(L)
$$

Plugging this back in gives

$$
S_{j}(L)=S_{f j}(L)+R S_{f j}(L)=(1+R) S_{f j}(L)
$$

Solving for $S_{f j}(L)$ gives

$$
S_{f j}(L)=\frac{1}{1+R} S_{j}(L)
$$

Then

$$
S_{r j}(L)=\frac{R}{1+R} S_{j}(L)
$$

This allows us to simplify (I.35)

$$
\begin{aligned}
\frac{\partial \bar{S}_{j}}{\partial t}+\frac{2 v}{L}\left[S_{f j}(L)-S_{r j}(L)\right] & =\bar{G}_{j} \bar{S}_{j}+\bar{S}_{p j} \\
\frac{\partial \bar{S}_{j}}{\partial t}+\frac{2 v}{L}\left[\frac{S_{j}(L)}{1+R}-\frac{R S_{j}(L)}{1+R}\right] & =\bar{G}_{j} \bar{S}_{j}+\bar{S}_{p j} \\
\frac{\partial \bar{S}_{j}}{\partial t}+\frac{2 v}{L}\left[\frac{1-R}{1+R}\right] S_{j}(L) & =\bar{G}_{j} \bar{S}_{j}+\bar{S}_{p j} \\
\frac{\partial \bar{S}_{j}}{\partial t}+\frac{2 v}{L}\left[\frac{1-R}{1+R}\right] \frac{S_{j}(L)}{\bar{S}_{j}} \bar{S}_{j} & =\bar{G}_{j} \bar{S}_{j}+\bar{S}_{p j}
\end{aligned}
$$

If we let

$$
\tau_{p}=\frac{L}{2 v}\left[\frac{1+R}{1-R}\right] \frac{\bar{S}_{j}}{S_{j}(L)}
$$

where $\tau_{p}$ is the photon decay rate, we get

$$
\frac{\partial \bar{S}_{j}}{\partial t}=\bar{G}_{j} \bar{S}_{j}+\bar{S}_{p j}-\frac{\bar{S}_{j}}{\tau_{p}}
$$

If we assume an average gain and exponential growth of the field we have

$$
S_{f j}(L) \approx S_{f j}(0) e^{\bar{G}_{j} L}
$$


We can use this value to calculate the average value of $S_{f j}$

$$
\begin{aligned}
\bar{S}_{f j} & =\frac{1}{L} \int_{0}^{L} S_{f j}(0) e^{\bar{G}_{j} z} d z \\
& =\frac{1}{L} \frac{S_{f j}(0)}{\bar{G}_{j}}\left(e^{\bar{G}_{j} L}-1\right)
\end{aligned}
$$

Using (I.41) to replace $S_{f j}(0)$ we get

$$
\begin{aligned}
\bar{S}_{f j} & =\frac{1}{L} \frac{S_{f j}(L)}{\bar{G}_{j}} e^{-\bar{G}_{j} L}\left(e^{\bar{G}_{j} L}-1\right) \\
& =\frac{S_{f j}(L)}{L \bar{G}_{j}}\left(1-e^{-\bar{G}_{j} L}\right)
\end{aligned}
$$

The total photon density

$$
\bar{S}_{j}=\bar{S}_{f j}+\bar{S}_{r j}
$$

so assuming symetrical waves $\left(\bar{S}_{f j}=\bar{S}_{r j}\right)$ and

$$
\begin{aligned}
\bar{S}_{j} & =2 \bar{S}_{f j} \\
& =2 \frac{S_{f j}(L)}{L \bar{G}_{j}}\left(1-e^{-\bar{G}_{j} L}\right)
\end{aligned}
$$

We can use (I.36) to replace $S_{f j}(L)$

$$
\begin{aligned}
\bar{S}_{j} & =2 \frac{S_{j}(L)}{(1+R) L \bar{G}_{j}}\left(1-e^{-\bar{G}_{j} L}\right) \\
\frac{\bar{S}_{j}}{S_{j}(L)} & =\frac{2\left(1-e^{-\bar{G}_{j} L}\right)}{(1+R) L \bar{G}_{j}}
\end{aligned}
$$

From (I.39) we get

$$
\begin{aligned}
\tau_{p} & =\frac{L}{2 v}\left[\frac{1+R}{1-R}\right] \frac{\bar{S}_{j}}{S_{j}(L)} \\
& =\frac{L}{2 v}\left[\frac{1+R}{1-R}\right] \frac{2\left(1-e^{-\bar{G}_{j} L}\right)}{(1+R) L \bar{G}_{j}} \\
& =\frac{1-e^{-\bar{G}_{j} L}}{v(1-R) \bar{G}_{j}}
\end{aligned}
$$

which can be contrasted with the classical photon lifetime [40]. The derivation is given in K.1.

$$
\tau_{p}=\frac{2 L}{v_{g} \ln (1 / R)}
$$




\section{I.2.4 S Equation}

If we use $S_{p i}=\beta_{s i} N / \tau_{n}$ then we obtain:

$$
\frac{\partial \bar{S}_{i}}{\partial t}=\bar{G}_{i} \bar{S}_{i}-\frac{\bar{S}_{i}}{\tau_{p}}+\frac{\beta_{s i} N}{\tau_{n}}
$$

where $\beta_{s i}$ is the portion of the spontaneous emission that goes into mode $i$.

\section{I.3 Phase equation}

$$
\begin{aligned}
& \frac{\partial E_{j}}{\partial t}+v \frac{\partial E_{j}}{\partial z}=-i \beta_{j} E_{j}+\bar{F}_{s j} \\
& \frac{\partial E_{j}}{\partial t}+v \frac{\partial E_{j}}{\partial z}=\left(g_{i}+i h_{i}\right) E_{j}+\bar{F}_{s j}
\end{aligned}
$$

We drop spontaneous emission which we can add later. Define:

$$
\begin{aligned}
\bar{G}_{i} \bar{E}_{j} & \approx \frac{1}{L} \int_{0}^{L} g_{i} E_{j} d z \\
\bar{H}_{i} \bar{E}_{j} & \approx \frac{1}{L} \int_{0}^{L} h_{i} E_{j} d z
\end{aligned}
$$

integrate over length and divide by $L$ :

$$
\begin{gathered}
\frac{1}{L} \int_{0}^{L}\left\{\frac{\partial E_{j}}{\partial t}+v \frac{\partial E_{j}}{\partial z}\right\}=\frac{1}{L} \int_{0}^{L}\left\{\left(g_{i}+i h_{i}\right) E_{j}\right\} \\
\frac{\partial \bar{E}_{j}}{\partial t}+\frac{v}{L}\left(E_{j}(L)-E_{j}(0)\right)=\bar{G}_{i} \bar{E}_{j}+i \bar{H}_{i} \bar{E}_{j}
\end{gathered}
$$

Assume a constant magnitude and phase perturbation in time (as did Henry I think):

$$
E_{j}(t)=E_{0 j} e^{i \phi(t)}
$$

We then get:

$$
\begin{aligned}
\frac{\partial}{\partial t}\left\{E_{0 j} e^{i \phi(t)}\right\}+\frac{v}{L}\left(E_{j}(L)-E_{j}(0)\right) & =\bar{G}_{j} \bar{E}_{j}+i \bar{H}_{j} \bar{E}_{j} \\
i \frac{\partial \phi}{\partial t} \bar{E}_{j}+\frac{v}{L}\left(E_{j}(L)-E_{j}(0)\right) & =\bar{G}_{j} \bar{E}_{j}+i \bar{H}_{j} \bar{E}_{j}
\end{aligned}
$$


If $h_{j}=0$ then $\bar{H}_{j}=0$ and $d \phi / d t=0$ :

$$
\frac{v}{L}\left(E_{j}(L)-E_{j}(0)\right)=\bar{G}_{i} \bar{E}_{j}
$$

subtracting from the propagation equation then gives:

$$
i \frac{\partial \phi}{\partial t} \bar{E}_{j}=i \bar{H}_{i} \bar{E}_{j}
$$

and we have:

$$
\begin{aligned}
\frac{\partial \phi}{\partial t} & =\bar{H}_{j} \\
& =\frac{1}{L} \int_{0}^{L} h_{j} E_{j} d z \\
& =\frac{1}{L} \int_{0}^{L}\left(\alpha_{h} \frac{A}{2}\left(N-N_{t r}\right)-\Re\left(K_{j}\right)\right) d z
\end{aligned}
$$

which gives:

$$
\left.\frac{\partial \phi}{\partial t}=\alpha_{h} \frac{A}{2}\left(\bar{N}-N_{t r}\right)-\frac{1}{L} \int_{0}^{L} \Re\left(K_{j}\right)\right) d z
$$

\section{I.4 Issues}

Spontaneous Emission Term: The derivation gives for the SPE term in the photon equation:

$$
S_{p f i}=F_{s i}^{*} E_{f i}+F_{s i} E_{f i}^{*}
$$

which can be contrasted with the RTE one:

$$
S_{p i}=\frac{\beta_{s i} \bar{N}}{\tau_{n}}
$$

for the TWM we use for the field:

$$
E_{s p}=\sqrt{\frac{\beta_{s i} \bar{N}}{\tau_{n}}}
$$

Carrier dependent $\kappa$ : From the consideration of frequency dependence of the gain $g_{i}$ we get:

$$
\kappa(z, t)=\frac{A}{2}\left(N(z, t)-N_{t r}\right)
$$

which gives a dynamic distributed value for $\kappa(z, t)$ due to the dependence on $N$. This has not been implemented. 
Photon Lifetime: The derivation of the photon equation provides a value for $\tau_{p}$ :

$$
\tau_{p}=\frac{L}{2 v}\left[\frac{1+R}{1-R}\right] \frac{\bar{S}_{j}}{S_{j}(L)}
$$

which is dependent on the photon density distribution and is quite different from the classical one.

$$
\tau_{p}=\frac{L}{v_{g} \ln (1 / R)}
$$

Refer to appendix K.1 for a derivation. 


\section{Appendix $\mathbf{J}$}

\section{Useful Wave Relations}

\section{J.1 Index of Refraction}

The index of refraction results in an apparent change in speed of a field in media. The goal is to account for this change.

\section{J.1.1 No Damping}

Each particular charge feels the effects of all the other moving charges. Simplify by ignoring the other charges which occurs when $n \simeq 1$ [8, p31-2]. In this very little light is reflected which further simplifies things.

$[8,(31.3)]$

$$
E_{s}=E_{0} \cos \left(\omega\left[t-\frac{z}{c}\right]\right)
$$

or $[8,(31.4)]$

$$
E_{s}=E_{0} e^{i \omega\left(t-\frac{z}{c}\right)}
$$

Without plate wave travels $\triangle z$ in time $\frac{\Delta z}{c}$. Note $v=\frac{s}{t}$.

With plate it appears to travel at $v=\frac{c}{n}$ and so travels $\triangle z$ in time $\frac{n \triangle z}{c}$.

The additional time is $\triangle t=\frac{n \triangle z}{c}-\frac{\triangle z}{c}=(n-1) \frac{\triangle z}{c}$.

Take into account extra delay through plate by replacing $t$ in $[8,(31.4)]$ with $(t-\triangle t)$ or $\left[t-(n-1) \frac{\triangle z}{c}\right]$ and get $[8,(31.5)]$

$$
\begin{gathered}
E_{\text {after_plate }}=E_{0} e^{i \omega\left[t-(n-1) \frac{\Delta z}{c}-\frac{z}{c}\right]} \\
E_{\text {after_plate }}=E_{0} e^{i \omega t-i \omega(n-1) \frac{\Delta z}{c}-i \omega \frac{z}{c}} \\
E_{\text {after_plate }}=e^{-i \omega(n-1) \frac{\Delta z}{c}} E_{0} e^{i \omega t-i \omega \frac{z}{c}}
\end{gathered}
$$

or $[8,(31.6)]$

$$
E_{\text {after_plate }}=e^{-i \omega(n-1) \frac{\Delta z}{c}} E_{0} e^{i \omega\left[t-\frac{z}{c}\right]}
$$


The effect of the plate is to multiply by an exponential term or add a phase to it. So the glass has retarded the field.

Given that $x$ is small $e^{x} \simeq 1+x$ and we can write [8, (31.7)]

$$
e^{-i \omega(n-1) \frac{\Delta z}{c}}=1-i \omega(n-1) \frac{\triangle z}{c}
$$

Using this in $[8,(31.6)]$ we get $[8,(31.8)]$

$$
\begin{aligned}
E_{\text {after_plate }} & =\left(1-i \omega(n-1) \frac{\triangle z}{c}\right) E_{0} e^{i \omega\left[t-\frac{z}{c}\right]} \\
& =E_{0} e^{i \omega\left[t-\frac{z}{c}\right]}-i \omega(n-1) \frac{\triangle z}{c} E_{0} e^{i \omega\left[t-\frac{z}{c}\right]} \\
& =E_{s}+E_{a}
\end{aligned}
$$

Think of the $E_{a}$ term as a complex number at right angles $(-i)$ to $E_{s}$. Therefore the real part of index of refraction is responsible for the change in speed of the field.

From $[8,(31.4)]$ rewritten $[8,(31.9)]$

$$
E_{s}=E_{0} e^{i \omega\left(t-\frac{z}{c}\right)}
$$

Right at plate $z=0$ gives $[8,(31.10)]$

$$
E_{s}=E_{0} e^{i \omega t}
$$

For an oscillator with resonant frequency $\omega_{0}[8,(31.11)]$

$$
m\left(\frac{d^{2} x}{d t^{2}}+\omega_{0}^{2} x\right)=F
$$

and for an electric field $[8,(31.12)]$

$$
F=q_{e} E_{s}=q_{e} E_{0} e^{i \omega t}
$$

$[8,(31.13)]$

$$
m\left(\frac{d^{2} x}{d t^{2}}+\omega_{0}^{2} x\right)=q_{e} E_{0} e^{i \omega t}
$$

Solution is $[8,(31.14)]$

$$
\begin{gathered}
x=x_{0} e^{i \omega t} \\
\dot{x}=i \omega x_{0} e^{i \omega t} \\
\ddot{x}=-\omega^{2} x_{0} e^{i \omega t}
\end{gathered}
$$

Substitute into $[8,(31.13)]$ 


$$
\begin{gathered}
m\left(\frac{d^{2} x}{d t^{2}}+\omega_{0}^{2} x\right)=q_{e} E_{0} e^{i \omega t} \\
m\left(-\omega^{2} x_{0} e^{i \omega t}+\omega_{0}^{2} x_{0} e^{i \omega t}\right)=q_{e} E_{0} e^{i \omega t} \\
m x_{0}\left(\omega_{0}^{2}-\omega^{2}\right)=q_{e} E_{0}
\end{gathered}
$$

giving $[8,(31.15)]$

and $[8,(31.16)]$

$$
x_{0}=\frac{q_{e} E_{0}}{m\left(\omega_{0}^{2}-\omega^{2}\right)}
$$

$E_{a}$ at $\mathrm{P}$ from $[8,(30.18)]$

$$
x=\frac{q_{e} E_{0}}{m\left(\omega_{0}^{2}-\omega^{2}\right)} e^{i \omega t}
$$

$$
\text { Total_field_at_P }=-\frac{\eta q_{e}}{2 \epsilon_{0} c} \dot{x}\left(t-\frac{z}{c}\right)
$$

Substitute in for $\dot{x}$ and $x_{0}$ gives [8, (31.17)]

$$
E_{a}=-\frac{\eta q_{e}}{2 \epsilon_{0} c} \dot{x}\left(t-\frac{z}{c}\right)=-\frac{\eta q_{e}}{2 \epsilon_{0} c}\left[i \omega \frac{q_{e} E_{0}}{m\left(\omega_{0}^{2}-\omega^{2}\right)} e^{i \omega\left(t-\frac{z}{c}\right)}\right]
$$

Compare to $E_{a}$ from $[8,(31.8)]$

$$
E_{a}=-i \omega(n-1) \frac{\triangle z}{c} E_{0} e^{i \omega\left[t-\frac{z}{c}\right]}
$$

and setting them equal

$$
\begin{aligned}
-\frac{\eta q_{e}}{2 \epsilon_{0} c}\left[i \omega \frac{q_{e} E_{0}}{m\left(\omega_{0}^{2}-\omega^{2}\right)} e^{i \omega\left(t-\frac{z}{c}\right)}\right] & =-i \omega(n-1) \frac{\triangle z}{c} E_{0} e^{i \omega\left[t-\frac{z}{c}\right]} \\
\frac{\eta q_{e}}{2 \epsilon_{0}}\left[\frac{q_{e}}{m\left(\omega_{0}^{2}-\omega^{2}\right)}\right] & =(n-1) \triangle z
\end{aligned}
$$

gives $[8,(31.18)]$

but $\eta=N \triangle z$ so $[8,(31.19)]$

$$
(n-1) \triangle z=\frac{\eta q_{e}^{2}}{2 \epsilon_{0} m\left(\omega_{0}^{2}-\omega^{2}\right)}
$$

$$
n=1+\frac{N q_{e}^{2}}{2 \epsilon_{0} m\left(\omega_{0}^{2}-\omega^{2}\right)}
$$

This must assume the approximation

$$
n \simeq 1+\frac{1}{2} \chi
$$

when $\chi \ll 1$. [9] 


\section{J.2 Dispersion}

\section{J.2.1 Gas}

In most ordinary gases the natural frequency of resonance $\omega_{0}$, corresponds to UV light which is much higher than for visible light. [8, p31-6] Ignoring $\omega$ in [8, (31.19)](J.2)

$$
n=1+\frac{N q_{e}^{2}}{2 \epsilon_{0} m\left(\omega_{0}^{2}-\omega^{2}\right)}
$$

we see that in gases $n$ will be nearly constant.

\section{J.2.2 Transparent Substances}

For most transparent substances like glass, $n$ behaves like in a gas. Note that as $\omega$ rises, taking a little bit more away from the denominator, the second term increases, and therefor $n$ will also. This explains why blue light bends more than red light.

\section{J.2.3 Opaque Materials}

Many materials (like graphite) which are opaque to visible light are transparent to x-rays. In this case we can set $\omega_{0}=0$ in $[8,(31.19)](\mathrm{J} .2)$

$$
n=1+\frac{N q_{e}^{2}}{2 \epsilon_{0} m\left(\omega_{0}^{2}-\omega^{2}\right)}
$$

giving

$$
n=1-\frac{N q_{e}^{2}}{2 \epsilon_{0} m \omega^{2}}
$$

Note that when $n<1$ the speed of the waves is faster than $c$ ! This just means that the phase shift which is produced by the scattered light can be either positive or negative. [8, p31-6]

\section{J.2.4 Damping and Multiple Oscillators}

To take into account the damping factor in the atomic oscillator we change the term $\left(\omega_{0}^{2}-\omega^{2}\right)$ to $\left(\omega_{0}^{2}-\omega^{2}+i \gamma \omega\right)$ in $[8,(31.19)](\mathrm{J} .2)$. When this is done for multiple oscillators it results in $[8,(31.20)]$

$$
n=1+\frac{q_{e}^{2}}{2 \epsilon_{0} m} \sum_{k} \frac{N_{k}}{\omega_{k}^{2}-\omega^{2}+i \gamma_{k} \omega}
$$

Plot is shown below. 


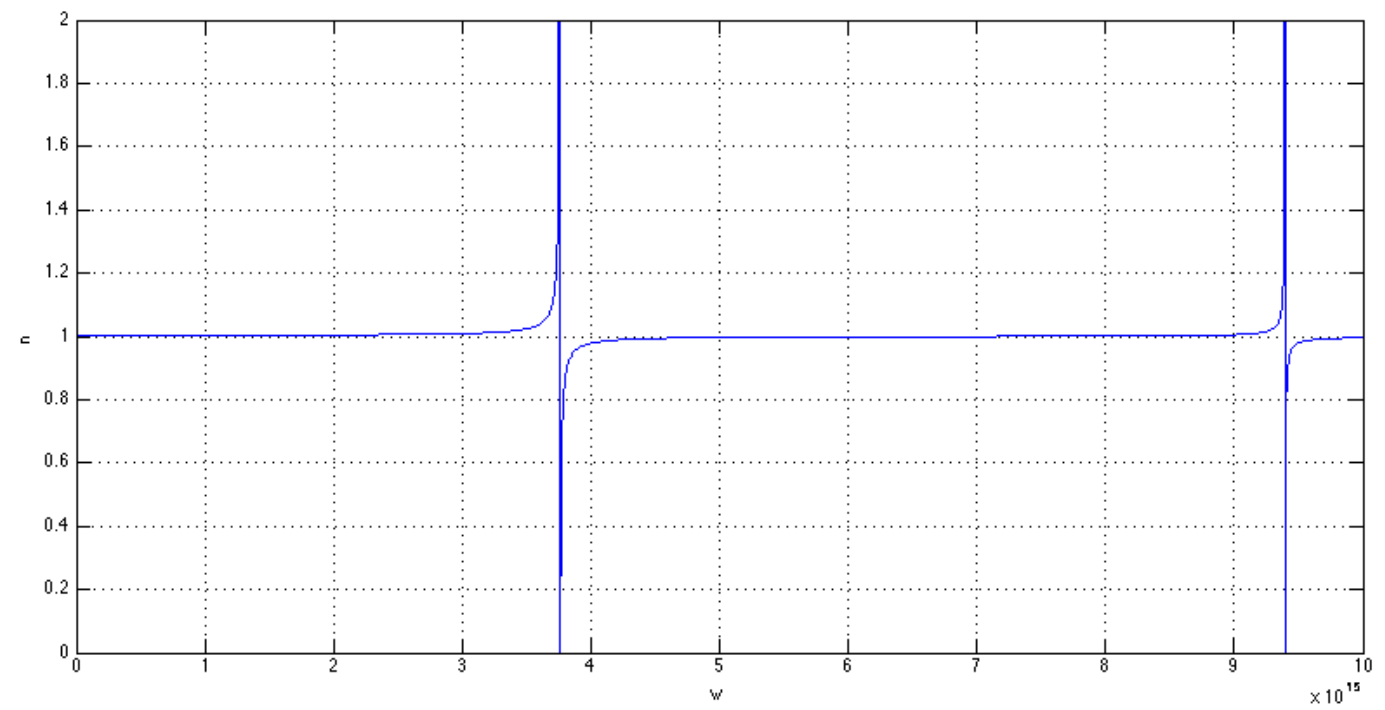

Figure J.1: Sample plot with two oscillators. $w$ in $H z$. 


\section{Appendix $\mathrm{K}$}

\section{Miscellaneous Derivations}

\section{K.1 Classic Photon Lifetime}

The photon lifetime estimate assumes a uniform electron density $N$ giving a gain

$$
G=g_{0}\left(N-N_{t r}\right)
$$

for the stimulated emission within the laser. The photon density then grows exponentially as

$$
S=S_{0} e^{2 \alpha z}
$$

where

$$
2 \alpha=\frac{G}{v_{g}}
$$

and $v_{g}$ is the group velocity; the velocity of the photon energy propagating from the left facet and right facet and back. In steady state the photons reflected back to the start in a single round trip $\left(\tau=\frac{2 L}{v_{g}}\right)$ must have the same density. Starting with $S_{0}$ photons at the left facet we will have

$$
\begin{aligned}
S\left(z_{2}\right) & =S_{0} e^{2 \alpha L} \\
& =S_{0} e^{\frac{G}{v_{g}} L}
\end{aligned}
$$

and $R_{2} S_{0} e^{\frac{G}{v_{g}} L}$ will be reflected back. This in turn means

$$
\begin{aligned}
S\left(z_{1}\right) & =R_{2} S_{0} e^{\frac{G}{v_{g}} L} e^{2 \alpha L} \\
& =R_{2} S_{0} e^{\frac{G}{v_{g}} L} e^{\frac{G}{v_{g}} L} \\
& =R_{2} S_{0} e^{\frac{2 G}{v_{g}} L}
\end{aligned}
$$


We then have with $R_{1} R_{2} S_{0} e^{\frac{2 G}{v_{g}} L}$ starting out again. Therefore for steady state we have

$$
\begin{aligned}
R_{1} R_{2} S_{0} e^{\frac{2 G}{v_{g}} L} & =S_{0} \\
R_{1} R_{2} e^{\frac{2 G}{v_{g}} L} & =1 \\
e^{\frac{2 G}{v_{g}} L} & =\frac{1}{R_{1} R_{2}} \\
\frac{2 G}{v_{g}} L & =\ln \left[\frac{1}{R_{1} R_{2}}\right] \\
G & =\frac{v_{g}}{2 L} \ln \left[\frac{1}{R_{1} R_{2}}\right]
\end{aligned}
$$

If we write the steady state averaged density rate equation (3.13) where we let $\Gamma g_{0}\left(N-N_{t r}\right)=$ $G$ we get

$$
\begin{aligned}
& \frac{d S}{d t}=G S-\frac{S}{\tau_{p}} \\
& \frac{d S}{d t}=\left(G-\frac{1}{\tau_{p}}\right) S
\end{aligned}
$$

At steady state this is 0 giving

$$
\begin{gathered}
\frac{d S}{d t}=\left(G-\frac{1}{\tau_{p}}\right) S=0 \\
G=\frac{1}{\tau_{p}}
\end{gathered}
$$

and therefore

$$
\begin{aligned}
\tau_{p} & =\frac{1}{G} \\
& =\frac{2 L}{v_{g} \ln \left[\frac{1}{R_{1} R_{2}}\right]}
\end{aligned}
$$

